\title{
OKLAHOMA
}

A Summary of Activities

of the

U.S. GEOLOGICAL SURVEY

WATER RESOURCES DIVISION

in

Fiscal Years 1986-87

Compiled by John S. Havens

U.S. GEOLOGICAL SURVEY

Open-File Report 88-172

Oklahoma City, Oklahoma

1988 


\author{
DEPARTMENT OF THE INIERIOR \\ DONAID PAUL HODEI, Secretary \\ UNITED STATES GEOLOGICAL SURVEY \\ Dallas I. Peck, Director
}

For additional information

write to:

District Chief

U.S. Geological Survey

Water Resources Division

215 Dean A. MoGee, Room 621

Oklahoma City, Oklahoma 73102
Copies of this report can be purchased from:

U.S. Geological survey Books and Open-File Reports Section Federal Center, Box 25425 Denver, Colorado 80225 


\section{CODES USED IN TABLES}

\section{Station Typo}

CS = Crest stage and discharge

CSR = Flood hydrograph and rainfal I (SR recorder)

LF = Low flow site

PR = Partial record site

QWD = Quality of water - daily

QWH = Quality of water - hourly

QWP = Quality of water - periodic

QWMP = Quality of water analyzed by another agency, published by USGS

RES = Reservoir gage operated and
published by UsGS
RESP = Reservoir gage operated by another
agency and publ ished by USGS
RESU = Reservoir gage unpubl ished
SED = Sediment sample collection
STGU = Stream gage only - unpublished
STR = Stream gage operated and
published by UsGS
STRP = Stream gage operated by another
agency and published by USGS

Stage Source

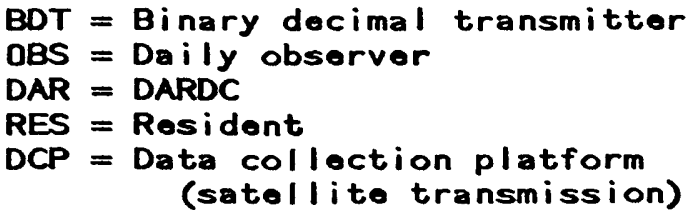

RES $=$ Resident

(satellite transmission)

$B=$ Biological

C = Chemical

$\mathbf{N}=$ Nutrients

$\mathbf{0}=$ Organic

$\mathbf{R}=$ Radiological

$S=$ Sediment

[Some parameters not collected at the frequency indicated by Station Typo.]

Cooperator (Coop)

$\mathbf{A}=$ Federal

$B=U . S$. Bureau of Reclamation

$C=U . S$. Corps of Engineers

D = Mt. Park Master Conservancy Dist.

$E=$ Oklahoma Conservation Commission

G = USGS Cooperative Matching Funds

$H=$ Oklahoma Dept. of Transportation

$J=$ Oklahoma Water Resources Board

$K=$ City of Oklahoma City

$L=$ Grand River Dan Authority

$M=$ Central Oklahoma Master Conservancy District*

$\mathbf{N}=$ Fort Cobb Master Conservancy District*
$P=$ City of Tulsa*

$Q=$ City of Altus*

$R=C i t y$ of Ada*

$S=$ City of Sapulpa*

$T=$ Lugert-Altus Irrigation District*

$U=$ City of Lawton*

$V=$ Foss Reservoir Master Conservancy

$W=$ Operated by a neighboring state

$X$ = Oklahoma Gas and Electric Company*

$\mathbf{Z}=$ City of Claremoro*

$b=$ National Park Service

$c=$ City of Norman

* Funds provided through the Oklahoma Water Resources Board. [Includes cooperators for period of record; some cooperators not active in FY86-87.]

Aquifer Symbols

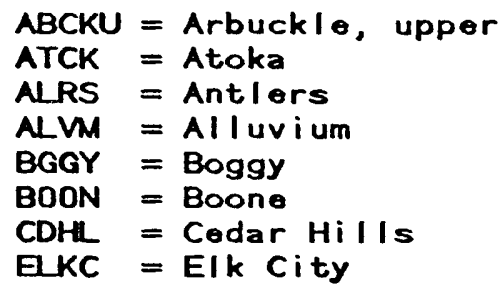

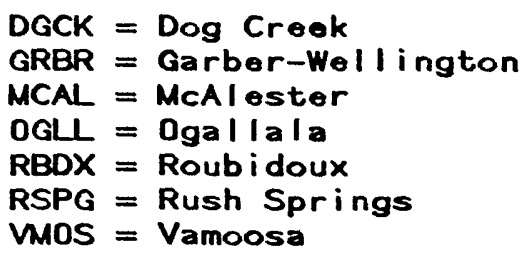

DGCK = Dog Creok

MCAL = McAlester

OGLL = Oga I I a I a

RSPG = Rush Springs

VMOS = Vamoosa 


\section{CONTENTS}

A message from the District Chief.................. I

U.S. Geological survey origin................... 3

Water resources mission of the U.S. Geological survey..... 5

Oklahoma District organization chart............... 6

Types of investigations and sources of funding

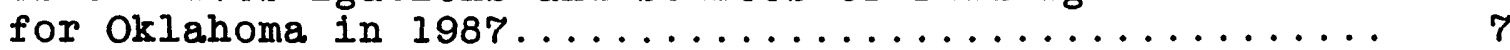

List of cooperators.......................... 8

Water conditions in oklahoma.................... 9

Summary of current and recently completed projects....... 11

OKOO1 -- Surface-water stations............... 11

OKOO2 -- Ground-water stations............... 11

OKOO3 -- Water-quality stations.............. 12

OK004 -- Sediment stations................. 13

OKOO5 -- NADP acid-rain monitoring stations........ 13

OKOO6 -- Limited detail flood insurance studies..... 14

OK007 -- Oklahoma water-use data system.......... 14

OK062 -- Central Midwest Regional Aquifer System

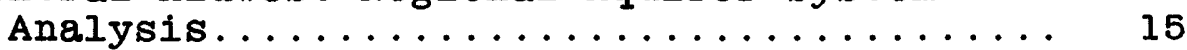

OK065 -- North Canadian River geohydrology......... 16

OK068 -- Geochemistry of the Tar Creek lead-zinc area in oklahoma................ 16

OK069 -- Oklahoma springs................ 17

OK070 -- Roubidoux aquifer model, Oklahoma......... 18

OKOT2 -- Cimarron alluvium and terrace model....... 18

OKOT3 -- Rush Springs-Marlow aquifer study......... 19

Ok074 -- Roubidoux aquifer hydrogeochemistry........ 20

OK075 -- Limnology of selected coal-mine ponds...... 21

OK076 -- Antlers aquifer model, Phase II......... 22

OK078 -- Water-level network, Oklahoma........... 22

OK079 -- Streamflow statistics for Oklahoma streams.. 23

OK080 -- Shale hydrogeology................. 24

OK081 -- Blaine aquifer study................ 25

OK082 -- Central Oklahoma (Garber-Wellington) aquifer National Water-Quality Assessment....... 26

OK083 -- Altus Air Force Base hydrology:

Reconnaissance and presurvey.......... 27

OK084 -- Hydrogeology Chickasaw National

Recreation Area.................. 28

OK085 -- Geographic information system software...... 29

Other District activities in support of the

State's water programs................... 30

Oklahoma reports by the U.S. Geological survey

and cooperating agencies.................... 32

Sources of U.S. Geological Survey publications

and information......................... 64

Codes used in tables.................... inside back cover 


\section{TABLES}

Table 1. Alphabetical listing of current and historical

Page gaging stations maintained by the U.S. Geolo-

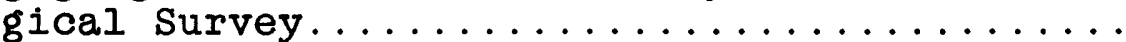

2. Station number listing of current and historical gaging stations maintained by the U.S. Geolo-

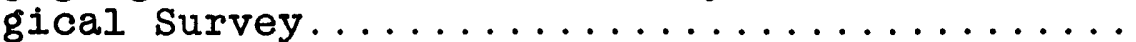

3. Continuous and partial record ground-water level sites currently measured in oklahoma....... 132

\section{ILLOSTRATIONS}

Figures 1-9 Maps showing:

1. Areas for which reconnaissance hydrologic studies have been made.............. 133

2. Average annual runoff in Oklahoma $1970-79 \ldots \ldots .134$

3. Location of principal aquifers in Oklahoma..... 136

4. Total 1985 ground-water and surface-water withdrawals by county................ 136

5. Locations of continuous-record stream-gaging stations....................... 137

6. Locations of water wells measured continuously, monthly, or quarterly............... 138

7. Number of water wells measured in each county during winter period................ 139

8. Locations of water-quality sampling sites..... 140

9. Locations of sediment sampling sites......... 141 


\title{
OKLAHOMA, A SUMMARY OF ACTIVITIBS \\ OF THE O.S. GBOLOGICAL SURVBY, WATER RESOURCES DIVISION, IN FISCAL YBARS 1986-87
}

Compiled by

John S. Havens

\begin{abstract}
This report summarizes the activities of the Oklahoma District, Water Resources Division, U.S. Geological Survey, for fiscal years 1986-87. Included are summary statements of current and recently completed projects, alphabetical and numerical listings of surface-water stations, and a bibliography of Oklahoma reports.
\end{abstract}

\section{A MESSAGB FROM THE DISTRICT CHIBF}

For approximately 50 years, the U.S. Geological Survey (USGS), Water Resources Division, has cooperated with State and local governments within Oklahoma in collecting and interpreting water-resources data for the benefit of the citizens of Oklahoma and the Nation. It has been my pleasure to have been selected as the new District Chief, effective August 1986. I am replacing James $H$. Irwin, who spent the past 20 years as a member of the USGS team in his home state of Oklahoma. A close relationship has been developed with State, local, and other Federal agencies to provide them with accurate and meaningful water information. I am proud of the contributions of the USGS and I plan to continue this relationship. As Oklahoma progresses, the demand for water-resources data will increase and the USGS will continue to play an important role in supplying these water-data needs.

Since the publication of our last Activities Report in 1985, a number of projects have been completed and new investigations started. Modeling studies of the the North Canadian River from Oklahoma City to Eufaula Lake and the Antlers aquifer in the southeastern part of Oklahoma have been completed. 
New projects and data collection programs will continue to be concentrated on what are considered to be the most important water problems in Oklahoma. Ongoing projects include hydrogeologic studies of the Cimarron terrace deposits and the Blaine Gypsum aquifer; a study of the hydraulic and physical properties of selected shaley formations in Oklahoma; and the Central Oklahoma National Water Quality Assessment (NAWQA) project, a study of the Garber-Wellington and associated aquifers. Feasibility studies of the hydrogeology of Chickasaw National Recreation Area and of probable hazards to the environment from possible contaminants at Altus Air Force Base have been started. New surface-water gaging installations are being equipped with water-quality monitors that will collect continuous temperature, dissolved oxygen, $\mathrm{pH}$, and specific conductance data. Approximately 90 of our gaging stations are equipped with data-collection platforms (DCP'S) which allow us to collect near real-time data. The data are transmitted through the GOES satellite and received by the District office computer.

This summary will be of help to those interested in the work of the USGS and its cooperating agencies. It provides a ready reference to U.S. Geological Survey publications describing the results of previous studies in Oklahoma, the current studies in the state, and the locations of the many sites where water information is being collected. To meet our State's needs for up-to-date reliable information on water resources, the USGS is proud to provide its know-how and expertise to meet this challenge.

Charles R. Burchett District Chief U.S. Geological Survey Oklahoma City, Oklahoma 


\section{D.S. GBOLOGICAL SURVBY ORIGIN}

The U.S. Geological Survey was established by an act of Congress on March 3 , 1879, to provide a permanent Federal agency to conduct the systematic and scientific "classification of the public land, and examination of the geological structure, mineral resources, and products of national domain." An integral part of that original mission includes publishing and disseminating the earth-science information needed to understand, to plan the use of, and to manage the Nation's energy, land, mineral, and water resources.

Since 1879, the research and fact-finding role of the USGS has grown and been modified to meet the changing needs of the Nation it serves. As part of that evolution, the USGS has become the Federal Government's largest earth-science research agency, the Nation's largest civilian map-making agency, the primary source of data on the Nation's surface- and ground-water resources, and the employer of the largest number of professional earth scientists. Today's programs serve a diversity of needs and users. Programs include:

- Conducting detalled assessments of the energy and mineral potential of the Nation's land and offshore areas.

- Investigating and issuing warnings of earthquakes, volcanic eruptions, landslides, and other geologic and hydrologic hazards.

- Conducting research on the geologic structure of the Nation.

- Studying the geologic features, structure, processes, and history of the other planets of our solar system.

- Conducting topographic surveys of the Nation and preparing topographic and thematio maps and related cartographic products.

- Developing and producing digital cartographic data bases and products.

- Collecting data on a routine basis to determine the quantity, quality, and use of surface and ground water.

- Conducting water-resource appraisals in order to describe the consequences of alternative plans for developing land and water resources.

- Conducting research in hydraulics and hydrology, and coordinating all Federal water data acquisition. 
- Using remotely sensed data to develop new cartographic, geologic, and hydrologic research techniques for natural resources planning and management.

- Providing earth-science information through an extensive publications program and a network of public access points.

Along with its continuing commitment to meet the growing and changing earth-science needs of the Nation, the USGS remains dedicated to its original mission to collect, analyze, interpret, publish, and disseminate information about the natural resources of the Nation--providing "Earth Science in the Public Service." 


\section{WATER RBSOURCES MISSION \\ OF THB O.S. GBOLOGICAL SURVBY}

The water resources mission of the U.S. Geological survey is to provide the hydrologic information needed by others to help manage the Nation's water resources. To accomplish its mission, the Survey, in cooperation with state and local governments and other Federal agencies:

- Collects data on a systematic basis to determine the quantity, quality, and use of surface and ground water, and the quality of precipitation.

- Conducts water resources investigations and assessments at national, State, and local scales, characterizes water resources conditions, and provides the capability to predict the impact on the resource of managerial actions, proposed development plans, and natural phenomena.

- Conducts basic and problem-oriented hydrologic and water-related research that is likely to produce knowledge useful for the resolution of water-resources problems facing the state, regions, and Nation.

- Acquires information useful in predicting and delineating water-related natural hazards from flooding, volcanoes, mudflows, and land subsidence.

- Coordinates the activities of all Federal agencies in the acquisition of water data, and operates water informaiton centers.

- Disseminates data and the results of investigations through reports, maps, and other forms of public release.

- Provides scientific and technical assistance in hydrology to other Federal agencies, to state and local agencies, to licensees of the Federal Energy Regulatory Commission, and, on behalf of the U.S. Department of state, to international agencies.

- Administers the provisions of the Water Resources Research Act of 1984 which include the state Water Resources Research Institute Program (Section 104) and the Water Resources Research Grant Program (Section 105). 


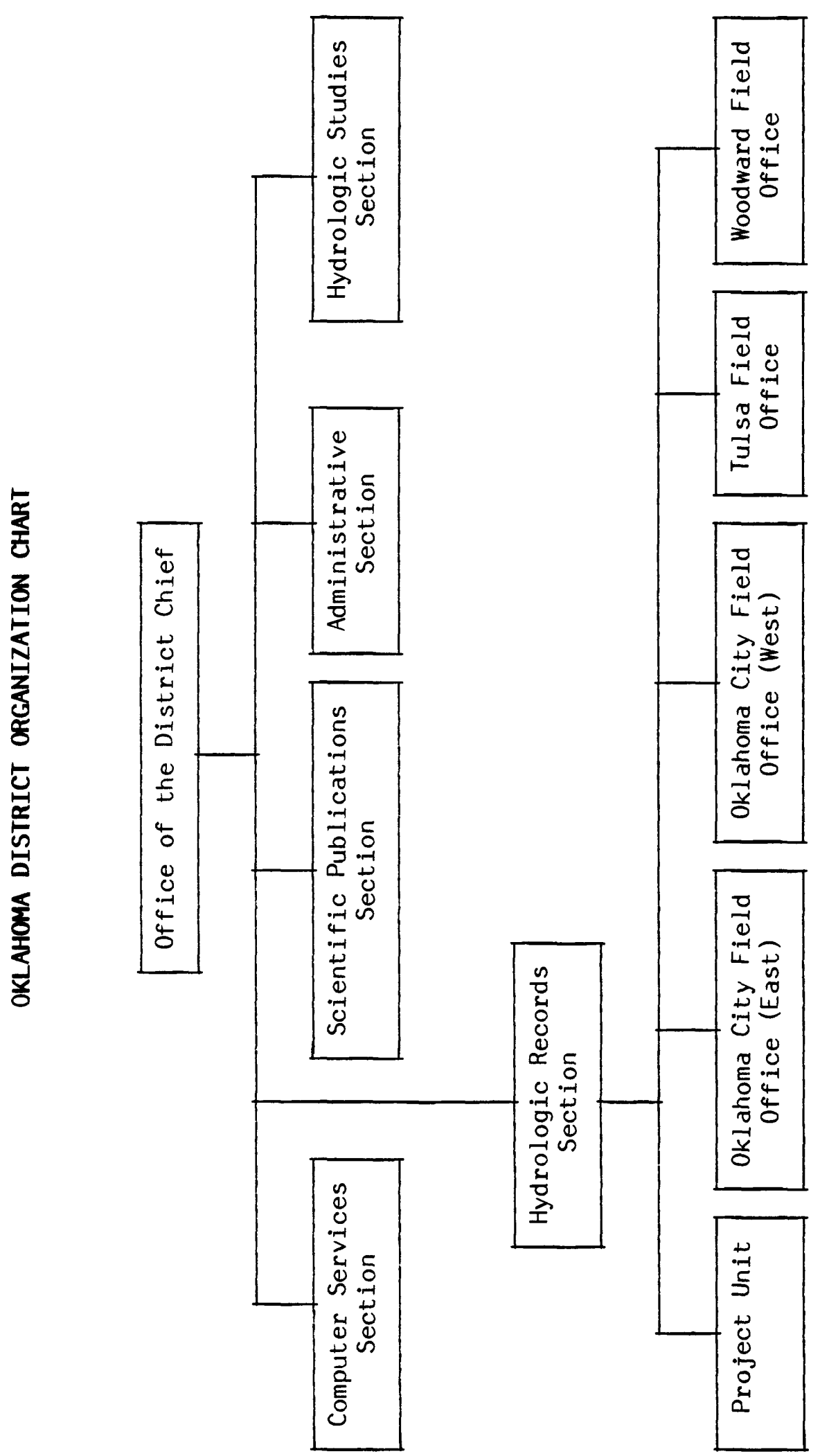




\section{TYPES OF INVESTIGATIONS AND SOURCES OF FUNDING FOR ORLAHOMA IN 1987}

Four broad categories of investigations are conducted in Oklahoma to obtain the information needed by managers and planners for the solution or alleviation of water problems in the State. These categories are: (1) Areal studies involving the appraisal of groundwater resources and river basins, (2) hydrologic data collection involving the statewide surface-water, groundwater, and quality-of-water monitoring programs, (3) research involving special studies that improve our understanding of hydrology, and (4) administration of programs involving the collection of data for national programs. The diagram below shows these investigations expressed as a percentage of the District's total work for fiscal year 1987 .

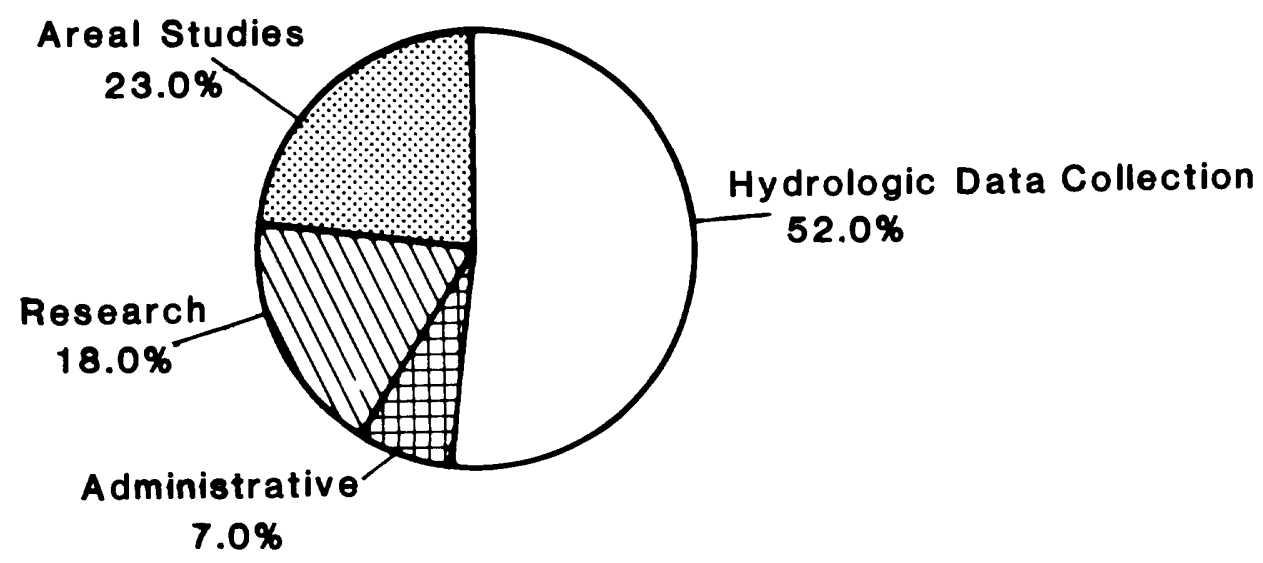

The investigations shown above are supported by services and funds from three basic programs: (1) Cooperative programs with 50 percent of the funds provided by state and local agencies and the remaining 50 percent by Federal funds, (2) Federal programs with funds appropriated directly to the U.S. Geological Survey, and (3) other Federal agency programs (OFA) supported entirely by other Federal agencies. In fiscal year 1987, the financial support for these three programs in Oklahoma was about $\$ 3,030,000$, distributed as follows:

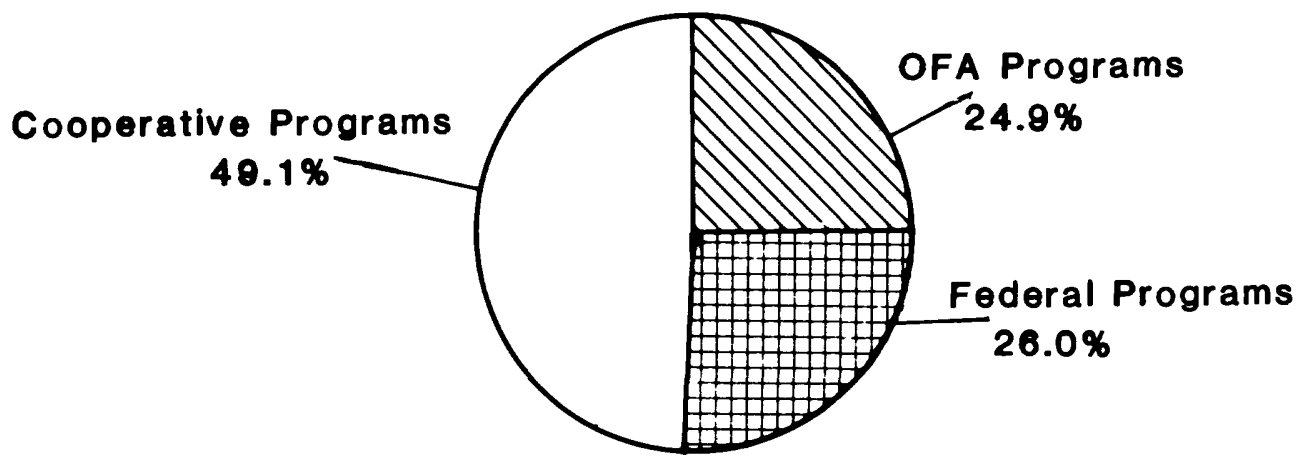




\section{LIST OF COOPERATORS}

The following table lists state, local, and other Federal agencies that supported water-resources investigations in cooperation with the U.S. Gelogical Survey during fiscal years 1986-87:

\section{State Agencies}

Oklahoma Geological Survey

Oklahoma State Health Department

Oklahoma Water Resources Board

Oklahoma Pollution Control Coordinating Board

Oklahoma Department of Transportation

\section{Local Agencies}

City of Ada

City of Altus

City of Lawton

City of Oklahoma City

City of Tulsa

Central Oklahoma Master Conservancy District

Ft. Cobb Reservoir Master Conservancy District

Foss Reservoir Master Conservancy District

Grand River Dam Authority

Lugert-Altus Irrigation District

Mountain Park Master Conservancy District

Oklahoma Gas and Electric Company

\section{Federal Agencies}

Federal Emergency Management Agency

U.S. Army Corps of Engineers

U.S. Bureau of Reclamation

National Park Service

U.S. Air Force 


\section{WATER CONDITIONS IN ORLAHOMA}

Oklahoma's surface and ground water range widely in their availability and quality across the State. The general characteristics of these water resources are described in a series of reconnaissance studies published as hydrologic atlases. (See list of Oklahoma reports.) Three of the atlases describe water conditions in Oklahoma Panhandle counties and the other nine atlases describe water conditions in two-degree quadrangles covering the remainder of the State (fig. 1).

Oklahoma's major aquifers include alluvium and terrace deposits, the Ogallala Formation (High Plains aquifer), and consolidated deposits that include limestone, sandstone, and gypsum (fig. 3). Significant ground-water declines have occured in some of the aquifers in the western part of the State because of extensive irrigation. This irrigation, particularly in the Panhandle, accounts for about 76 percent of the ground-water withdrawals in Oklahoma.

The distribution of water continues to be one of the major water-resource problems in Oklahoma. In general, surface water of adequate quality for most of man's uses is abundant in the eastern half of the State (fig. 2) while the western half is deficient in surface water. The eastern counties rely predominantly on surface water while the western counties rely predominantly on ground water (fig. 4).

Recent discoveries of potentially toxic chemicals in lakes, streams, and aquifers at various places in the United States has stimulated wide-spread concern for the chemical quality of the Nation's water resources, particularly ground water. The troublesome chemicals generaliy have been trace metals and man-made organic compounds. In Oklahoma, as nearly everywhere, few data are available to determine if these chemicals have been introduced into the State's surface and ground waters. The Geological survey and other Federal and State agencies are expanding their efforts to identify water-quality problems, but the effort required to investigate even the major streams and aquifers throughout Oklahoma will be very large. The Oklahoma District recently began an extensive assessment of the water quality of the Central Oklahoma (Garber-Wellington) aquifer, but few wells will be sampled until summer, 1988 .

Another major problem for Oklahoma is periodic flooding, which can occur almost anywhere in the State. Two extreme flood events occurred in Oklahoma during the 1987 water year. During September and October 1986, a major cold front associated with Hurricane Payne settled over the State and heavy rainfall produced greater than 100-year floods in the 
northeast, north-central, and central parts of the state. In May 1987, floods occurred in the southwest, south-central, and central parts of the state.

Water-resources problems in Oklahoma have no easy solutions. The problems can be solved only by long-term comprehensive planning and management, which require reliable hydrologic information. As the state's population increases, an increasing demand will be placed on Oklahoma's water resources. The current activities of the Oklahoma District address many of the state's problems described above. These activities, which are described in the following pages, are designed to provide hydrologic data and related information necessary for the best utilization and management of both Oklahoma's and the Nation's water resources. 
Title: SURFACE-WATER STATIONS

Leader: Hauth, Leland D.

Number: OKOO1

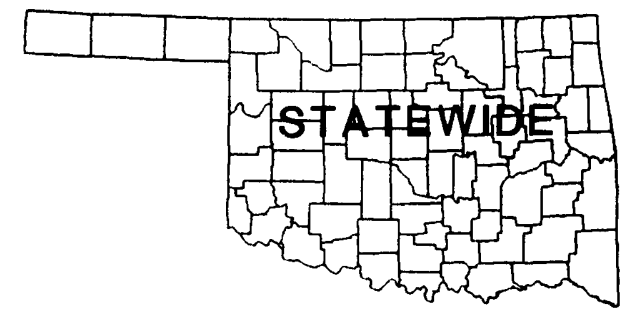

Problem: Surface-water information is needed for purposes of surveillance, planning, design, hazard warning, operation, and management, in water-related fields such as water supply, hydroelectric power, flood control, irrigation, bridge and culvert design, wildlife management, poliution abatement, flood-plain management, and water resources development. To provide this information an appropriate data base is necessary.

Objective: A. To collect surface-water data for:

(1) Assessment of water resources, (2) operation of reservoirs or industries, (3) forecasting, (4) disposal of wastes and pollution controls, (5) discharge data to accompany

water-quality measurements, (6) compact and legal requirements, and (7) research or special studies. B. To collect data for analytical studies of the statistical properties of, and trends in, the occurrence of surface water.

Progress: Surface-water data were collected at 186 active sites: 119 stream-gaging stations (continuous-discharge records), 30 lake gages, 7 crest-stage gages (peak-discharge records), 2 dual-digital gages (synchronous stage-rainfall with discharge hydrograph data only), 1 l continuous stage-only gages, 2 continuous stage with high-flow discharge data only, and 2 miscellaneous discharge data sites ( $f i g .5$ ). In addition, continuous records of discharge were computed and published from 13 gaging stations operated and maintained by the U.S. Army Corps of Engineers.

Cooperating Agencies: See codes at end of report for "COOP" given in tables 1 and 2 .

Title: GROUND-WATER STATIONS

Leader: Hauth, Leland D.

Number: OKOO2

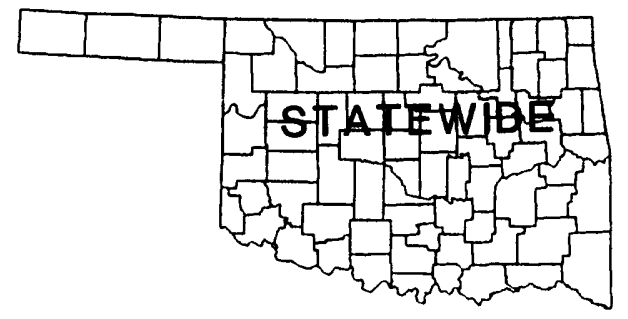

Problem: Long-term water-level records are needed to evaluate the effects of climatic variations on the recharge to and discharge from the State's aquifers, to provide a data base with which to measure the effects of development, and to provide data for management of the resource. 
Objective: A. To collect water-level data to provide long-term records of the general response of the hydrologic system to natural climatic variations and induced stresses. B. To provide a data base against which the short-term records acquired in areal studies can be analyzed.

Progress: During the 1987 fiscal year, water levels were measured in 1,074 wells: continuous water levels were monitored at 44 sites and yearly, monthly, or quarterly measurements were made at 1,030 other sites ( $f i g .6$ and 7 ).

Cooperating Agency: Oklahoma Water Resources Board

Title: WATER-QUALITY STATIONS

Leader: Hauth, Leland D.

Number: OKOO3

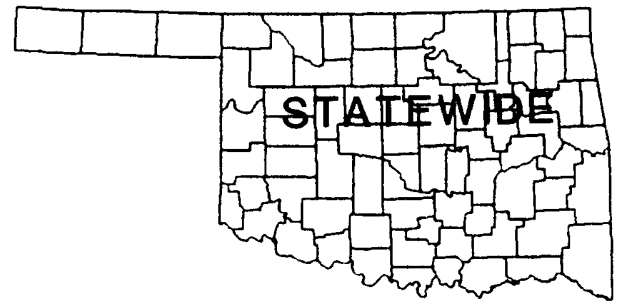

Problem: Water-resource planning and water-quality assessment require a nationwide data base of relatively standardized information. For intelligent planning and realistic assessment of water resources, chemical and physical qualities of rivers and streams must be defined and monitored.

Qbjective: To collect, analyze, and publish water-quality data for use by Federal, state, and local agencies involved in the planning and management of the state's water resources, and to provide data for Federal management of interstate and

international waters.

Progress: Water-quality data were collected at 38 active sites: of these, 7 were equipped with minimonitors for collection of continuous data. The sites are listed in tables 1 and 2 and their locations are shown on figure 8 . Five additional four-parameter mini-monitors were installed in Bird Creek.

Cooperating Agencies: Oklahoma Water Resources Board, Oklahoma Department of Health. See codes at end of report for "COOP" given in tables 1 and 2 . 
Title: SEDIMENT STATIONS

Leader: Hauth, Leland D.

Number: OK004

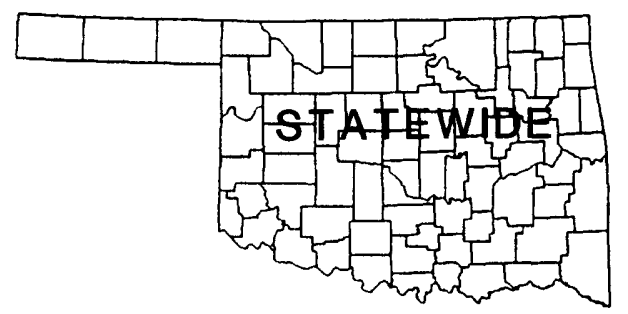

Problem: Water-resource planning and water-quality assessment require a nationwide data base of relatively standardized information. For intelligent planning and realistic assessment of water resources, sediment concentrations and discharges in rivers and streams must be defined and monitored.

Objective: To collect, analyze, and publish sediment data for use by Federal, State, and local agencies involved in the planning and management of the state's water resources, and to provide data for Federal management of interstate and international waters.

Progress: Sediment data are collected presently at 38 sites. These sites are listed in tables 1 and 2 and are identified under the "Water Quality Parameter" column as "S". Figure 9 shows the location of each site.

Cooperating Agencies: See codes at end of report for "COOP" given in tables 1 and 2 .

Title: NADP ACID-RAIN MONITORING STATIONS; NATIONAL TRENDS NETWORK (NTN) FOR MONITORIN

Leader: Kurklin, Joanne $\mathrm{K}$.

Number: OKO05

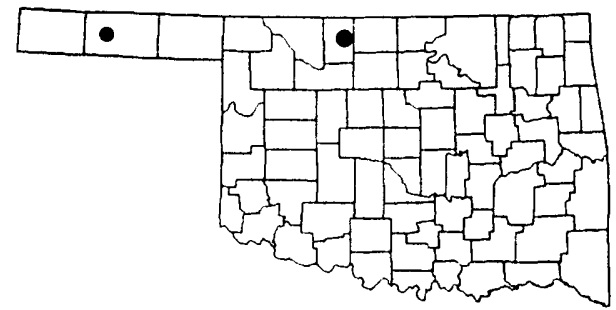

Problem: To establish long-term monitoring stations to detect and measure levels of atmospheric deposition.

objective: To determine variations in atmospheric depositions that occur on a week-to-week basis. To collect wet and dry deposition products for analysis of elements and compounds that can contribute to chemical composition of surface waters.

Progress: Two active continuous sites are maintained. 
Title: FEMA FLOOD INSURANCE STUDIES BY LIMITED DETAIL METHODOLOGIES

Leader: Tortorelli, Robert L.

Number: OK006

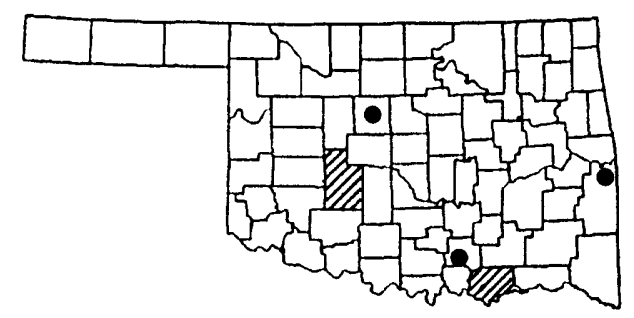

Problem: The 100-year recurrence-interval flood potential needs to be determined for the purposes of the National Flood Insurance Program (NFIP) in many populated areas. Because of limited funding, alternative ways of determining the 100-year profile and boundaries are being used--limited-detail methodologies.

Objective: The 100-year recurrence-interval flood will to be published as inundation maps and flood profiles for: Dover, Pocola, Tishomingo, and Bryan and Caddo Counties, Oklahoma, by use of limited detail study (LDS) methods.

Progress: The Limited-Detailed Study/Flood Insurance Studies (LDS/FIS) for Tishomingo, Pocola, and Bryan County, Oklahoma are complete. Dover is complete except for the report. The LDS/FIS for Caddo County, Oklahoma, which includes stream reaches near seven communities, was coordinated and planned.

Title: OKLAHOMA WATER-USE DATA SYSTEM Leader: May, Jayne $\mathrm{E}$. Number: OKOO'7

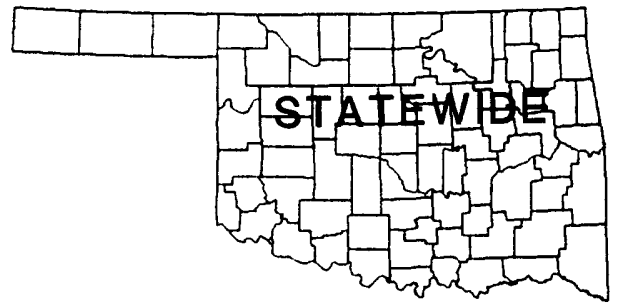

Problem: Water-use data that have been collected for the state of Oklahoma are presently distributed throughout many Federal, State, and local agencies. The data are in different formats and contain different bits of information that may make the data from one agency unusable by another agency. Also, many of the available data represent aggregated water use for multiple-source water systems, rather than for single wells or stream withdrawals.

Objective: To begin the development of a comprehensive point-source water-use data collection and management system for Oklahoma.

Progress: The primary emphasis in the Oklahoma Water-Use Project for 1987 was writing the Oklahoma section of the 1987 National Water Summary (NWS) which will focus on water supply and use. In the past, the emphasis of this project has been on aggregating and processing annual reported water-use values for the National Water Use Data System (NWUDS). Beginning in 1987, the emphasis was on improving the water-use data collected 
through interpretive studies. Two project proposals, one focusing on public-supply use and another on irrigation-water use, were written and submitted for consideration.

TitIe: CENTRAL MIDWEST REGIONAL AQUIFER SYSTEM ANALYSIS IN OKLAHOMA

Leader: Christenson, Scott C. Number: OK062

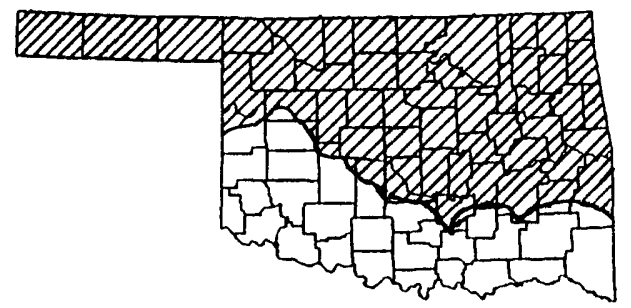

Problem: Mesozoic and Paleozoic formations are major sources of water supplies in some parts of oklahoma. In other parts of the state these formations contain saline water or brines. Some of the formations are oil and gas reservoirs and, at other locations, are storage reservoirs for industrial waste. A knowledge of the geohydrologic system is essential to determine the availability of the ground water and to plan maximum and orderly development of this vital resource.

Objective: (1) Describe the hydrologic system, including aquifer designation, hydraulic characteristics, and quality of the water within the regional aquifers. (2) Create a data base including water use, water levels, lithologic logs, geophysical logs, and chemical analyses of water samples. (3) Describe historic, present, and future problems associated with use of water. (4) Evaluate aquifer system responses to future conditions.

Progress: The Central Midwest Regional Aquifer System Analysis (CMRASA) project is complete except for the final report. Two reports received Director's approval in 1987, and the final report is in review. A presentation of the results of the project was made at the Oklahoma Water Resources Conference in September 1987. 
Title: GEOHYDROLOGY OF THE ALLUVIUM AND TERRACE DEPOSITS OF THE NORTH CANADIAN RIVER FROM OKLAHOMA CITY TO EUFAULA LAKE, CENTRAL OKLAHOMA

Leader: Havens, John S.

Number: OK065

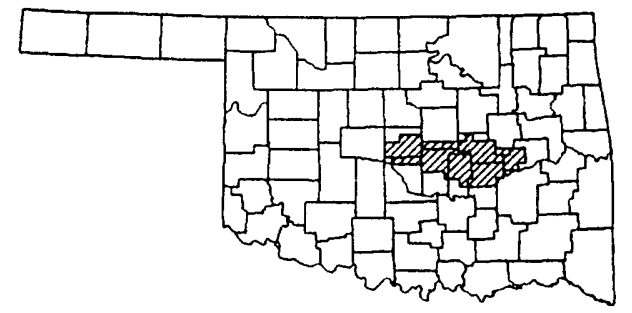

Problem: Ground water in the alluvial and terrace deposits of the North Canadian River is used for irrigation, municipal, stock, and domestic supplies. Increasing demand has made it necessary for the state to formulate a plan to manage this resource. Quantitative knowledge of the hydrologic system is necessary for proper management.

Objective: The project is an investigation designed to provide quantitative knowledge of the hydrologic system necessary to manage the aquifer effectively. Specific objectives of the project are: (1) To describe the geologic setting of the alluvial and terrace deposits along the North Canadian River, (2) to provide a quantitative description of the hydrologic system, and ( 3 ) to compute the maximum annual yield from the aquifer based on a minimum twenty-year life span.

Progress: The basic data report for the project was published as Open-File Report (OFR 84-808). The final report for the project has been written and is in review.

Cooperating Agency: Oklahoma Water Resources Board

Title: GEOCHEMISTRY OF THE TAR CREEK LEAD-ZINC AREA IN OKLAHOMA

Leader: Parkhurst, David L.

Number: $0 K 068$

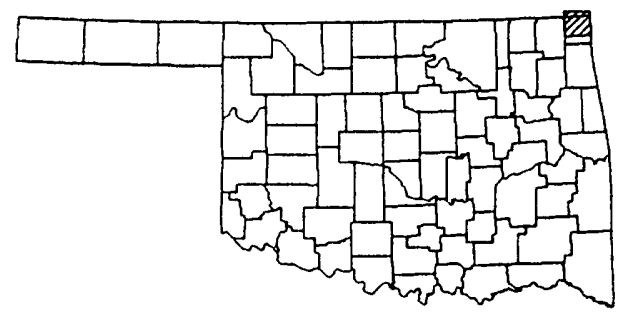

Problem: Large concentrations of zinc, lead, and cadmium are found in the water in abandoned zinc mines of northeastern Oklahoma. The water is draining into Tar Creek and the Grand Lake $\mathrm{O}^{\prime}$ the Cherokees. Also, the potential exists for downward migration of contaminants from the mines to the Roubidoux aquifer, an important water source for the area.

Objective: (1) To critically evaluate the thermodynamic data necessary to apply geochemical models to the zinc-cadmium-lead system. (2) To determine the geochemical reactions occurring 
with these metals in the abandoned mines and in the surface water. (3) To model the chemical reaction of mine water mixing with ground water of the Roubidoux aquifer.

Progress: The water-analyses data have been published as Open-File Report 87-453. The sediment-analyses data report is in final preparation before review. Analysis of discharge and metal loads from the abandoned mines has been completed, culminating in a presentation at the 1987 Oklahoma Water Resources conference at Oklahoma State University. Project complete except report.

Title: AN INVENTORY OF OKLAHOMA SPRINGS

Leader: Goemaat, Robert L.

Number: $0 K 069$

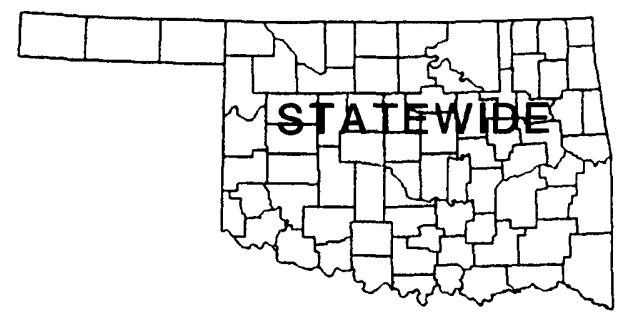

Problem: The flow in many of Oklahoma's streams is augmented in part by springflow. State agencies interested in water rights, ground water contribution to base flows, or streamflow accountability require knowledge of the location, quality and quantity of flow - particularly in areas where large springflows occur. An annotated state-wide springs report does not exist.

Objectives: (1) Do a physical inventory of Oklahoma springs; (2) measure the discharge, $\mathrm{pH}$, temperature and specific conductance; and (3) establish the relation between springflow and ground-water levels.

Progress: The project was suspended in 1986 due to lack of funds.

Cooperating Agency: Oklahoma Geological Survey 
Title: DIGITAL MODEL ANALYSIS OF THE ROUBIDOUX AQUIFER IN NORTHEASTERN OKLAHOMA

Leader: Christenson, Scott C. Number: OK070

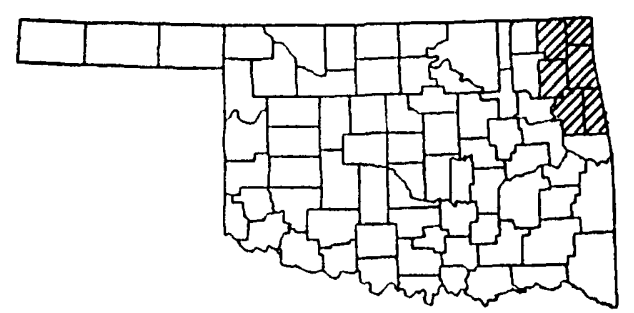

Problem: Water from the Roubidoux aquifer is used for public supplies and industrial purposes. Water users in the area are concerned about declining water levels as a result of withdrawal of water from the aquifer and the possibility of contamination of the water supply from abandoned lead and zinc mines in the northern part of the area. The possibility exists for downward migration of toxic mine waters through existing or abandoned leaky well casings or through fractures in rocks that overlie the Roubidoux aquifer.

Objective: The hydraulic properties of the formations above and below the Roubidoux aquifer are unknown. Information about the hydraulic properties of the rocks in the deep formations is needed for a better understanding of the direction and rates of ground-water flow in the Roubidoux aquifer and overlying geologic units.

Progress: The project is complete except for the report. The results of this study are being included with the results of the Roubidoux aquifer reconnaissance.

Titie: GEOHYDROLOGY OF ALLUVIUM AND TERRACE DEPOSITS OF THE CIMARRON RIVER FROM NEAR THE KANSAS STATE LINE TO GUTHRIE, OKLAHOMA

Leader: Adams, Gregory P.

Number: $\quad$ OKO72

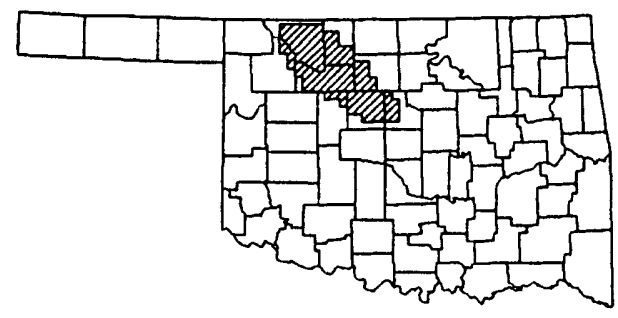

Problem: Ground water in the alluvium and terrace deposits along the Cimarron River in northwestern Oklahoma is used extensively for irrigation, municipal, stock, and domestic supplies. Increasing demand for water from these deposits makes it necessary to have a quantitative knowledge of the hydrologic system for formulation of effective management plans. The area of study extends about 115 miles from near Freedom to Guthrie, Oklahoma. This area includes the Cimarron Terrace and associated aquifers.

Qbjective: (1) To describe the geologic setting of the alluvial and terrace deposits along the Cimarron River from Freedom, Oklahoma to Guthrie, Oklahoma; (2) to estimate the quantity of water in storage and the annual recharge and the discharge from the alluvium and terrace deposits of the 
Cimarron River; (3) to provide estimates of the effects of ground-water withdrawal from the aquifer by means of a digital model of the aquifer-river system; and (4) to identify sources of existing and potential natural saline pollution.

Progress: A comprehensive work plan has been completed for the project. Outlines for the data and interpretive reports have been prepared. Two continuous water-level recorders were estabilished near the Cimarron River to provide aquifer diffusivity data. A monthly water-level network of 57 observation wells was established to record fluctuations in water levels during the study. In September 1987 a low-flow seepage measurement was conducted on the Cimarron River project area; several samples were collected to be analyzed for common ions and trace constituents. Approximately 60 percent of historical ground-water data for domestic, municipal, commercial, industrial, and irrigation wells has been entered in Ground-Water Site Inventory (GWSI). Hydrographs for water-level network wells and multiple-record wells have been compiled.

Cooperating Agency: Oklahoma Geological Survey

Title: NUMERICAL SIMULATION OF SATURATED THICKNESS AND STORAGE CHANGES RESULTING FROM PROJECTED PUMPING FROM THE RUSH SPRINGS-MARLOW AQUIFER, SOUTHWESTERN OKLAHOMA

Leader: Morton, Robert B. Number: $\quad$ OK073

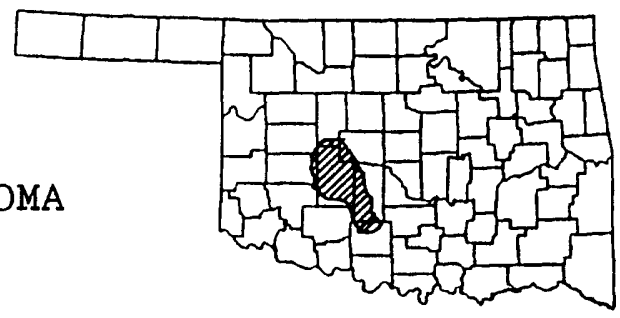

Problem: Since 1950, an estimated 1,500 large capacity irrigation wells have been drilled in the Rush Springs-Marlow aquifer. Well densities in the most intensively developed areas may be as much as four or five wells per square mile. The future economy of the area is dependent upon the sensible use and management of the water resources that are derived mostly from the Rush springs-Marlow aquifer.

Objective: The proposed study will give a better understanding of the hydrogeology of the Rush Springs-Marlow aquifer; will analyze the effect of present and future pumping on the aquifer; and will aid in the management and use of the Rush Springs-Marlow aquifer.

Progress: A base map was prepared. Maps showing the elevation of the potentiometric surface and the elevation of the base of the aquifer were completed. Ground-water samples were collected for quality-water analysis from 138 wells. The median dissolved-solids value is $450 \mathrm{mg} / 1$ based on specific conductance readings. Site-schedule data for the 138 sample 
sites plus site-schedule data for 63 earlier ground-water sites have been entered in Ground water SIte INventory (GWSI). The project has been suspended due to the lack of funding.

Cooperating Agency: Oklahoma Geological Survey

Title: HYDROGEOLOGIC AND GEOCHEMICAL STUDY OF THE ROUBIDOUX AQUIFER IN THE VICINITY OF THE PICHER MINING FIELD, NORTHEASTERN OKLAHOMA Leader: Christenson, scott C. Number: OK074

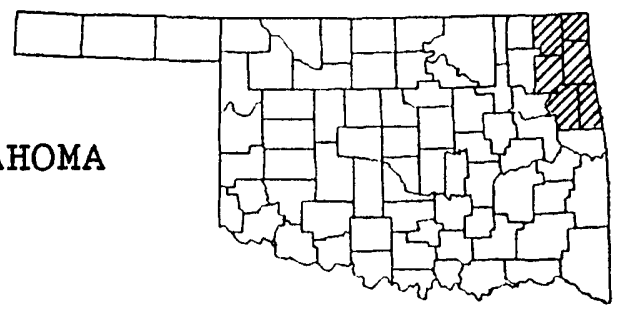

Problem: Most of the water supply for extreme northeastern Oklahoma is obtained from fractured sandstone and dolomite units in the Roubidoux Formation and associated formations of Cambrian and Ordovician ages. The demand for water from the principal aquifer, the Roubidoux, is expected to increase in proportion to the population growth of the area. There is concern that the Roubidoux, which averages 150 feet thick and lies between 800 and 1,000 feet below land surface, may be subject to contamination from abandoned mines of the Picher field. Water in the underground lead-zinc mines contains large concentrations of iron, zinc, cadmium, and lead. The contaminated water may migrate from the mines to the Roubidoux through abandoned water wells. A multi-agency effort to locate and plug all such wells began in late 1984. Participation in this effort will provide information in support of other ongoing investigations of the hydrogeology of the Roubidoux aquifer and of the geochemical mechanisms involved in the contamination of surface and ground waters in the vicinity of the Picher mining field.

Objective: To determine the geologic, hydraulic, and chemical characteristics of the Roubidoux Formation and of the overlying formations that separate the Roubidoux from the mined interval. Specific objectives are to: (1) Obtain a suite of geophysical logs for each abandoned well prior to plugging; recondition selected abandoned wells in order to construct a production well and several observation wells; (3) perform aquifer tests to determine hydraulic properties and leakage characteristics of the Roubidoux; and (4) collect water samples for chemical and isotope analyses to determine the geochemical evolution and age of water in the Roubidoux.

Progress: Attempts at converting old Roubidoux production wells into monitoring wells were unsuccessful. Many of the old wells could not be cleared to the Roubidoux Formation, and the wells that could be cleared were not located in positions that were suitable for an aquifer test. Therefore, the aquifer tests and sampling of wells could not be done. The wells that were cleared were logged with the District's geophysical 
logger. A DISPLA program was written to plot the digitized geophysical logs in report-ready format. Project complete except report.

Cooperating Agency: Oklahoma Water Resources Board

TitIe: IIMNOLOGY OF SELECTED COALMINE PONDS IN THE COAL-MINING REGION OF EASTERN OKLAHOMA

Leader: Parkhurst, Renee S.

Number: OK075

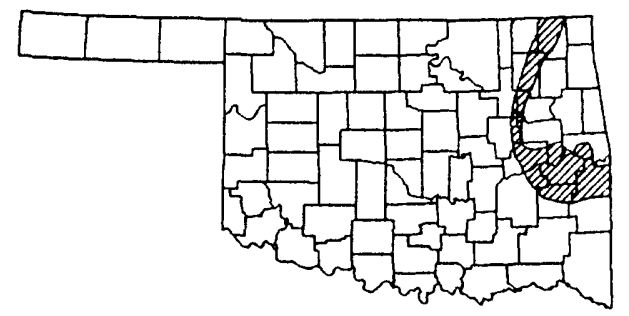

Problem: Information is not available on the limnological characteristics of mine ponds or other man-made ponds in the coal-mining region of eastern oklahoma. Limnological information is needed to manage this water resource created by strip mining, and to further understand the limnological processes occurring in mine ponds.

Objective: (1) Describe the limnological characteristics of the strip-mine ponds and other ponds in the area not associated with coal mining. (2) Determine if the limnological

characteristics of strip-mine ponds are significantly different from those of other ponds. (3) Determine if the limnological characteristics of strip-mine ponds are significantly different among (a) those associated with different coal seams, (b) those associated with different mining and reclamation practices and (c) those of different ages. (4) Intensively study selected strip-mine ponds and non strip-mine ponds to develop an understanding of hydrologic, chemical, and biological processes occurring within the ponds as well as the interrelationships among these processes.

Progress: The final sampling for the project was completed in 1987. All available data have been entered into computerized data files. Some preliminary statistical analyses have been made. Bathometric maps have been completed for one-third of the ponds. The rest of the maps are being completed. A report on the suitability of the strip-mine ponds for various uses is in review. The final interpretive report is being prepared.

Cooperating Agency: Oklahoma Geological Survey 
Title: SIMULATED EFFECTS OF PROJECTED

WATER USE ON THE ANTLERS

AQUIFER, SOUTHEASTERN OKLAHOMA

Leader: Morton, Robert B.

Number: OKO76

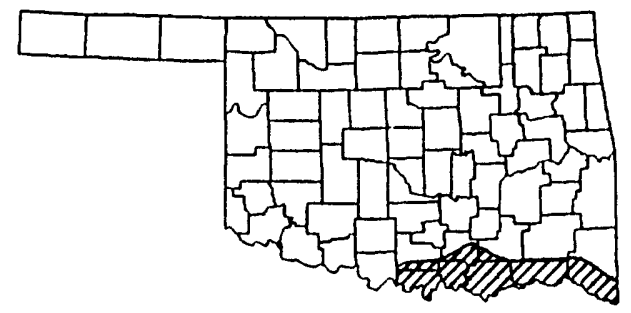

Problem: The Antlers Sandstone (Lower Cretaceous underlies an area of about 4,400 square miles in southeastern Oklahoma. In terms of volume of water in storage, the Antlers is one of the State's most important sources of fresh water. As ground-water supplies in other parts of the state become fully appropriated or become unsuitable for use due to lift requirements or quality considerations, the impetus for interbasin transfer of Antlers water will mount. The U.S. Army Corps of Engineers (USCOE) has made water-use projections for the area that span a period of several decades. The Corps has asked the

U.S. Geological Survey (USGS) to simulate the effects of the projected water use on the Antlers aquifer.

Objective: Simulate the effects that projected water use would have on the Antlers aquifer and present the results in a format that is readily usable by water-management agencies. The specific objectives are to: (1) Complete the data base assembled for earlier studies by incorporating new stratigraphic and hydrologic data; (2) adapt previously constructed data matrices, with appropriate changes in boundary conditions and parameter clarification for use with the modular ground-water model; (3) perform model simulations using projected water withdrawal rates provided by the Corps; and (4) present the results of the simulations as maps and tables that depict pertinent hydrologic information for each decennial year from 1990 to 2040 .

Progress: The final report has been submitted for Headquarters approval.

Cooperating Agency: U.S. Army Corps of Engineers

Title: ANALYSIS OF THE GROUND-WATER LEVEL OBSERVATION-WELL NETWORK FOR OKLAHOMA

Leader: Runkle, Donna L.

Number: OK078

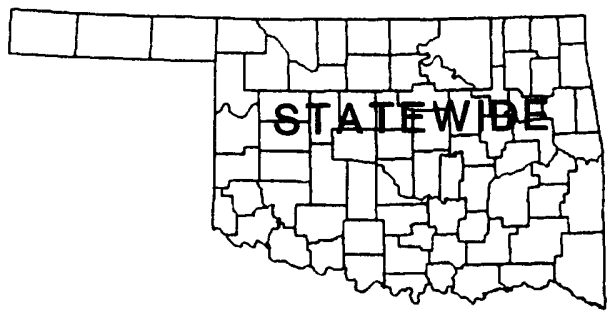

Problem: The U.S. Geological Survey (USGS) and Oklahoma Water Resources Board cooperatively operate a state-wide ground-water-level observation-well network that, in 1984 , consisted of 1,083 wells, 37 of which are measured 
continuously. Nearly all the rest are measured annually. About 40 percent of the wells are located in the four oklahoma counties that are underlain by the Ogallala aquifer. In contrast, 29 of the remaining 73 counties have only one or two network wells. The disproportionate distribution of wells has occurred mainly because the the ground-water network project has lacked a detailed, carefully considered set of objectives. Additions to and deletions from the network generally are made each year, but a sound rationale for doing so was not always apparent.

Objective: (1) Establish specific objectives for the ground-water level monitoring network. (2) Determine the optimum number and location of wells that are needed to meet those objectives. (3) Evaluate the existing network to see which wells should be retained according to this determination. (4) Develop a plan to remedy the deficiencies in the reworked network to bring it into compliance with the newly developed network objectives.

Progress: An extensive literature search was conducted on network design/analysis; a project work plan and an annotated outline of report and figures was prepared; a base map for study was ordered; possibilities were explored for using kriging methods to thin out dense water-level network wells, yet maintain optimum data collection. The project was suspended due to lack of funding.

Cooperating Agency: Oklahoma Water Resources Board

Title: STREAMFLOW STATISTICS FOR OKLAHOMA STREAMS

Leader: Tortorelli, Robert L. Number: OK079

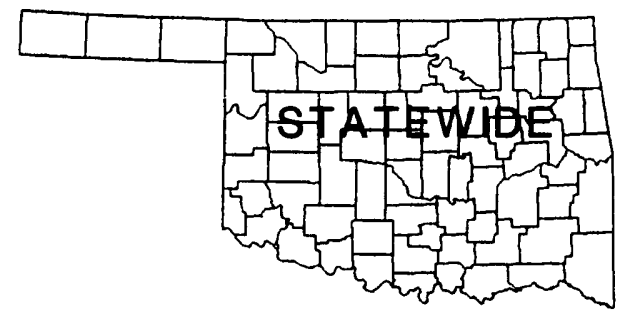

Problem: Most statistical data on streamflows in Oklahoma have not been published using a common period of record. Ten years of additional data have been collected since the last statistical publication. More current statistics are needed for use in design problems.

Objective: Compute streamflow statistics using most current data and publish in one publication.

Progress: Statistical summaries of streamflow at 148 gaging stations are presented in the final report. Summaries are presented for all stations having at least 10 years of data. Streamflow records for 70 of the 148 stations include regulated periods and data for these periods were analyzed separately to account for changes in streamflow due to regulating structures. For each gaging station, a brief description of the location, period of record, and drainage area is given. For those 
stations with regulated streamflow, a brief regulation history is given. This information is followed by tables of monthly and annual flow statistics, low- and high-flow frequency statistics, peak-flow frequency statistics, and flow-duration statistics. Also, daily flow-duration hydrographs are included for most stations. The final report received Director's approval in September. Project is completed.

Title: HYDROGEOLOGIC CHARACTERISTICS OF SELECTED SHALEY FORMATIONS IN OKLAHOMA

Leader: Overton, Myles D. Number: $\quad$ OK080

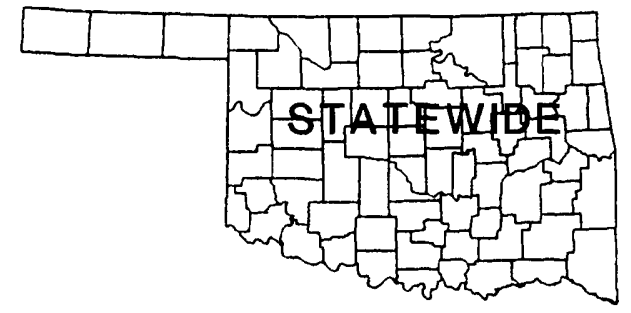

Problem: American industry produces millions of tons of potentially hazardous waste each year. In Oklahoma, industrial wastes are disposed of by near-surface burial and subsurface injection. Only one burial site currently is licensed in Oklahoma, but pressure is mounting for the selection and approval of other sites. In a recent reconnaissance study of the geology of rocks in Oklahoma that may be suitable for the disposal of hazardous waste, it was concluded that thick shales would be most favorable for near-surface burial of wastes. Few data are available, however, on the hydraulic properties of shales and little is known of the role of shales in ground-water flow systems.

Objective: (1) Conduct literature search on the hydrology of shales; (2) determine which physical properties of shale may be used as a relative index of permeability and fracture tendency; (3) select four representative shales for study, map the outcrop areas, and describe the detailed geology of rocks at the test sites; (4) evaluate the hydraulic and selected physical properties of the shales; (5) evaluate the fracture tendency of shales and the resulting effect on ground-water flow; (6) using digital models, estimate rates of ground-water flow through shales under realistic boundary conditions; and (7) suggest hydrogeologic and physical criteria or guidelines for use in evaluating the suitability of shales for waste disposal.

Progress: All necessary equipment has been received for slug testing. Core sampling and slug testing has been done at three of the four selected sites. Core samples have been delivered to the laboratory for physical analysis and determination of hydraulic conductivity. Programs to simplify development of slug-test curves and plotting of geophysical-log data have been developed to reduce analysis time.

Cooperating Agency: Oklahoma Geological Survey 
Title: HYDROGEOLOGY OF THE BLAINE AQUIFER AND ASSOCIATED UNITS IN SOUTHWESTERN OKLAHOMA

Leader: Runkle, Donna L. Number: OK081

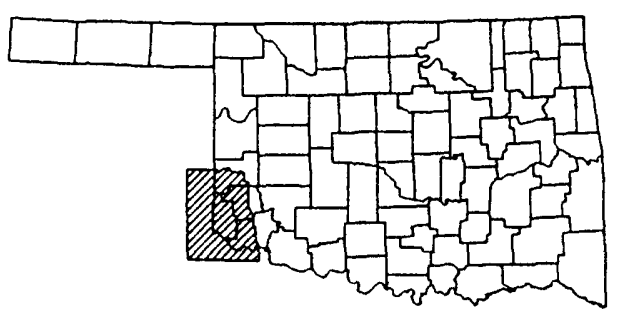

Problem: The Blaine aquifer consists of cavernous gypsum and dolomite beds interlayered with shales in the Permian Blaine Formation in Harmon, Jackson, and Greer Counties in southwestern Oklahoma. Ground water from the Blaine supports a local agriculture based mainly on irrigated cotton, corn, and wheat. Declining water levels in parts of the Blaine aquifer are evidence that water is being withdrawn at rates greater than it is being replenished. To help alleviate the situation, the Blaine aquifer has been artificially recharged since about 1961. The aquifer is recharged at more than 60 different locations by diverting streamflow and storm runoff into sink holes and wells. Water in the Blaine aquifer is not used for human consumption because of the large dissolved-solids concentrations. The calcium sulfate type water in the Blaine is acceptable for irrigation of salt-tolerant crops.

Objective: (1) Evaluate and map the stratigraphy and structure of the Blaine Formation and all geologic units in the study area; (2) evaluate the hydrology of the Blaine Formation and adjacent units; (3) determine the distribution of major and selected trace and organic (agriculturally applied) chemical constituents in the aquifers; (4) analyze the effects that extensive irrigation development and the artificial recharge program have had on the quantity and quality of water in the Blaine and associated aquifers; and (5) determine if opportunities exist for additional artificial recharge.

Progress: Historical ground-water, water-quality, and streamdischarge data have been compiled; aerial inventory of irrigation wells was completed; a monthly measured water-level network of over 125 wells was established; 11 continuous water-level recorders and 5 precipitation gages were installed; vibration time totalizers were installed on 25 wells to collect water-use data; a seepage run was conducted; discharge measurements were made at 7 sites on major streams; 24 test holes were drilled for geologic, water-level, and water-quality data; water samples were collected from wells and the water was analyzed for common ions, trace metals, and selected radiochemical and organic compounds; and geophysical logs were run on 25 open wells.

Cooperating Agencies: Oklahoma Water Resources Board, Oklahoma Geological Survey 
TitIe: CENTRAL OKLAHOMA

(GARBER-WELLINGTON) AQUIFER

NATIONAL WATER QUALITY

ASSESSMENT (NAWQA) PROGRAM

Leader: Christenson, Scott $\mathrm{C}$.

Number: OK082

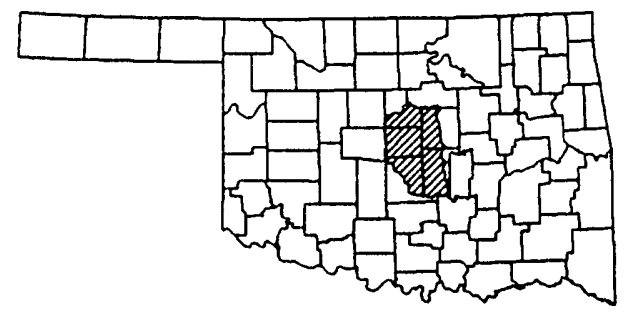

Problem: The Garber-Wellington aquifer and associated alluvial and terrace aquifers (collectively referred to as the Central Oklahoma aquifer) underlies about 3,000 square miles of central Oklahoma and is used for municipal, industrial, and domestic water supplies for most of the metropolitan area outside of Oklahoma City proper. Although pumpage from the aquifer has increased dramatically in recent years and water levels have declined, the principal threat to the continued viability of the Garber-Weliington aquifer is water-quality degradation. Salt water upconing in heavily pumped areas, natural occurrences of radioactive elements, and scattered occurrences of arsenic, chromium, and selenium in excess of the primary drinking-water standards are not uncommon. Urban development over much of the aquifer, particularly on the unconfined recharge area, has had major impacts. Infiltration of polluted urban runoff, leakage from underground storage tanks (containing hydrocarbons and industrial chemicals), percolation from poorly designed and closely spaced septic systems, and disposal of industrial waste are among the "urbanization" problems. Other problems are artifacts of central Oklahoma's history of oil and gas production--seepage from waste pits, leakage through defective well casing, improper brine disposal, and cross-contamination in improperly plugged or unplugged abandoned wells.

Objective: The Central Oklahoma (Garber-Wellington) Aquifer National Water Quality Assessment (NAWQA) has two related objectives. The first objective is to study water-quality problems that affect the beneficial uses of ground water within the study area. The second objective is to develop, test, and improve methods for performing regional assessments of ground-water quality, with an emphasis on nation-wide transfer value of the methods. Special importance will be placed on: (1) The design of the sampling strategy, and (2) the method used to estimate the likelihood and extent of water-quality problems.

Progress: A planning report was given Director's approval and published. A detailed work plan was developed and approved. Data describing the water quality of the Central Oklahoma aquifer have been collected from many sources and have been placed on the Oklahoma District's computer. Water samples have been collected for geochemical investigations.

Structure-contour maps have been prepared for the major strata in the study unit. A ground-water model has been prepared. Soil samples were collected for solid-phase analysis. 
Title: ALTUS AIR FORCE BASE HYDROLOGY: RECONNAISSANCE AND PRESURVEY PHASE

Leader: Stoner, Jerry D. Number: OK083

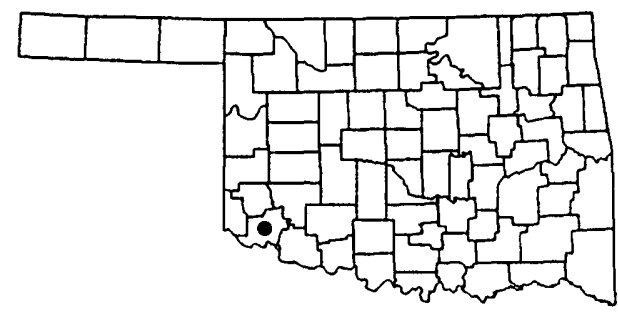

Problem: Altus Air Force Base (AFB) is among the priority sites scheduled for hazardous waste cleanup as part of the Installation Restoration Program (IRP) of the U.S. Air Force. Three sites on the Base were identified during the IRP Phase I study as having potentially hazardous waste. Two sites are fire training areas that may have been repositories for virtually any kind of combustible waste, liquid or solid, since the Base was established during WWII. The other site is a fuel depot that has been subject to an unknown volume of jet fuel spillage and leakage. The Base does not overlie a major aquifer. The Permian shales of the Hennessey Group underlie all but the extreme northern part of the Base. A few feet of Quaternary terrace deposits overlie the Hennessey in that area. The Air Force must assure that whatever contaminants have entered the ground do not migrate from the immediate vicinity of the disposal sites.

Objective: This study is a preliminary investigation, or a "presurvey," to determine the scope of work, and the associated costs, that would be required to conduct a comprehensive assessment of the degree of contamination at the sites, the areal extent of contamination, the ultimate fate of the contaminants in the hydrosphere, and the probable health risks associated with their existence. The final product of this study will be a report that details an investigative approach and the anticipated costs to conduct such an assessment.

Progress: The "presurvey" report was completed and submitted to the U.S. Air Force (USAF). The U.S. Air Force prepared a Statement of Work for the Installation Restoration Program Phase II stage 1 study at Altus AFB. The Phase II study is expected to begin at the end of FY87. The "Presurvey" project is completed.

Cooperating Agency: U.S. Air Force 
Title: PRELIMINARY INVESTIGATION OF THE HYDROGEOLOGY OF THE CHICKASAW NATIONAL RECREATION AREA

Leader: Hanson, Ronald L. Number: $0 K 084$

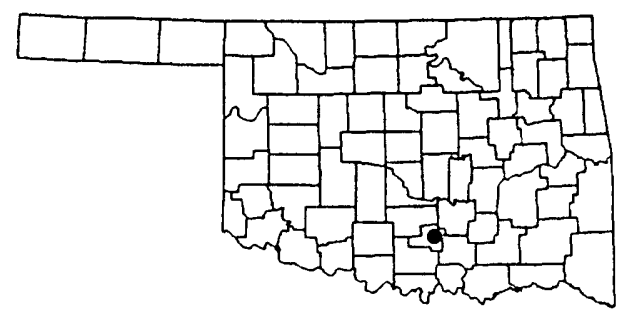

Problem: Spring and stream discharges in the Chickasaw National Recreational Area, located in south-central Oklahoma, have decreased significantly. In 1906, 33 springs flowed in the Park benefitted from 33 flowing springs but in 1987 only 5 springs have sustained flow. Reduced discharge from these springs and periodic cessation of flow in the two streams flowing through the Park have been attributed to increased pumping from the Simpson-Arbuckle aquifer that underlies the Park. Recorded ground-water level declines in the area suggest that two major springs are expected to dry up within 10 years and that all remaining springs in the Park will cease to flow within 40 years. Increased stresses on the system are anticipated when eight new wells upgradient from the Park become operational.

Objective: To collate the hydrogeologic data presently available for the Simpson-Arbuckle Aquifer and overlying surface waters in the Park and to evaluate the adequacy of these data for fully describing the surface-water/ground-water system associated with the area. If this evaluation shows that a more comprehensive study is warranted, a detailed study plan will be prepared describing the approach necessary to obtain a complete understanding of the system and the projected impacts of changes on the system as a result of potential hydrologic stresses imposed on the system.

Progress: All available reports describing the geology and hydrology of the area have been read and studied. The data include precipitation, streamflow, ground-water levels, surface-water quality, and ground-water quality data. The existing information shows that a more detailed study is required to adequately describe the hydrologic system in and adjacent to the Park. Four detailed project proposals---each outlining different levels of work effort but having scientific integrity in and of themselves--have been prepared and submitted to the National Park Service (NPS) for their consideration. A report describing the results of this preliminary investigation is being prepared. Project complete except report.

Cooperating Agency: National Park Service 
Title: FACILITATING THE ANALYSIS OF GROUND-WATER SYSTEMS USING A GEOGRAPHIC INFORMATION SYSTEM

Leader: Scott, Jonathon C.

Number: OKO85

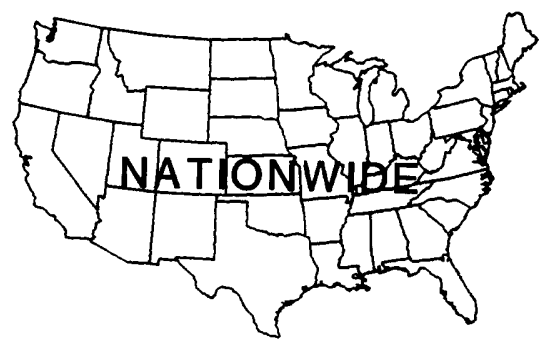

Problem: Ground-water hydrologists need sophisticated computer software to aid them in the analysis of ground-water systems. Geographic Information Systems (GIS) provide powerful map-making and analysis capability, however, system-integration programming is needed to transform this software into a productive tool.

Objective: (1) Develop software to transport water-quality and ground-water site-inventory data in the Geographic Information System (GIS). (2) Develop software to choose a set of arealy distributed sampling locations for a stratified set of polygons describing the study area. (3) Develop software to assist in visualizing the results of finite-difference numerical simulations using the Modular Ground-Water Flow Model. (4) Develop software to aid in the establishment of relations between land use and ground-water quality using statistical methods.

Progress: Software development has been completed for three of the four objectives. These products are now being used and tested. Transport of data from the National Water Information System (NWIS) into a Geographic Information System (GIS), transport of contoured data into a GIS, and an algorithm for stratified random sampling have been prepared. 


\section{OTHER DISTRICT ACTIVITIES IN SUPPORT OF THE STATE'S WATER PROGRAMS}

As part of the Geological Survey's responsibility to provide hydrologic information to all water users, the Survey participates in numerous other activities in addition to the regular Federal and state cooperative programs of hydrologic data collection and analysis. These other activities include involvement in various water-related committees and task forces, the review of technical reports on hydrology prepared by other agencies and universities, answering requests for hydrologic data and related information, and presenting information to schools, civic groups, and other interested groups on the water resources of oklahoma and the Nation. Some of the current special activities are:

Committee Activities--Members of the Oklahoma District staff participate in various technical committees and task forces dealing with water problems. Included are: (1) The Governor's Coordinating Committee on Water Resources Research;

(2) a ground-water committee to develop water-quality standards for Oklahoma's major aquifers; (3) a water-quality advisory board that reviews the state's ambient surface-water quality monitoring network, selects stream- and lake-sampling sites, determines constituents sampled, and establishes sampling frequency; (4) the Board of Trustees of the Applied systems Institute, a non-profit corporation established to promote scientific and educational information and research and development in meteorology and hydrology; (5) three interstate river compact commissions; (6) a Rural Abandoned Mine Program (RAMP) committee, which selects for reclamation abandonded surface mines based on their hazard to life and health; and (6) an American Society of Civil Engineers' task committee on water requirements of native vegetation.

Special Activities--Beginning in 1984 the Geological Survey published the first of an annual series of Water-Supply Papers, "National Water Summary", describing the conditions, trends, availability, quality, and use of the Nation's water resources. Each of these reports contains a chapter devoted to oklahoma. In addition to yearly hydrologic events, the reports address: water issues (1983), selected water-quality trends and ground-water resources (1984), surface-water resources (1985), ground-water quality (1986, unpublished), and water supply and demand (1987, unpublished).

During water year 1987, considerable effort was devoted to collecting flood data for two separate floods during the period from September 25 to October 8, 1986. Discharges for these events exeeded the 100-year recurrence interval at many sites in the central, south-central, and northeastern parts of the 
State. Many reservoirs in northeastern Oklahoma operated and maintained by the U.S. Corps of Engineers, Tulsa District, were filled to near capacity by the september 25 to October 8 flooding, and ensuing releases caused flooding downstream. The Oklahoma District, as a result of this flooding, also began upgrading many streamflow gaging stations, in cooperation with the Corps of Engineers, at sites above and below major reservoirs in eastern Oklahoma so that even during a "probable maximum flood," stage and discharge records may be obtained. 
ORLAHOMA REPORTS BY U.S. GEOLOGICAL SURVEY AND COOPERATING AGENCIES

List of categories:

U.S. GEOLOGICAL SURVEY PROFESSIONAL PAPERS

U.S. GEOLOGICAL SURVEY WATER-SUPPLY PAPERS

U.S. GEOLOGICAL SURVEY HYDROLOGIC ATLASES

U.S. GEOLOGICAL SURVEY MISCELLANEOUS GEOLOGIC INVESTIGATIONS

U.S. GEOLOGICAL SURVEY OPEN-FILE REPORTS

U.S. GEOLOGICAL SURVEY WATER-RESOURCES INVESTIGATIONS REPORTS

U.S. GEOLOGICAL SURVEY FEMA FLOOD REPORTS

U.S. GEOLOGICAL SURVEY WATER-RESOURCES DATA REPORTS (ANNUAL REPORTS)

U.S. GEOLOGICAL SURVEY CIRCULAR

U.S. GEOLOGICAL SURVEY ANNUAL REPORTS

U.S. GEOLOGICAL SURVEY MISCELLANEOUS REPORTS

OKLAHOMA WATER RESOURCES BOARD

OKLAHOMA WATER RESOURCES BOARD HYDROLOGIC INVESTIGATIONS

OKLAHOMA GEOLOGICAL SURVEY BULLETINS

OKLAHOMA GEOLOGICAL SURVEY CIRCULARS

OKLAHOMA GEOLOGICAL SURVEY MINERAL REPORTS

OKLAHOMA GEOLOGICAL SURVEY GEOLOGIC MAPS

OKLAHOMA GEOLOGICAL SURVEY EDUCATIONAL PUBLICATION

OKLAHOMA GEOLOGICAL SURVEY HYDROLOGIC ATLASES

OKLAHOMA GEOLOGICAL SURVEY HOPPER AND GEOLOGY NOTES

OKLAHOMA GEOLOGICAL SURVEY SPECIAL PUBLICATIONS

PROFESSIONAL SOCIETIES' ABSTRACTS AND MISCELLANEOUS REPORTS

MISCELLANEOUS

\section{U.S. GEOLOGICAL SURVEY PROFESSIONAL PAPERS}

Bedinger, M.R., and Sneigocki, R.T., 1976, Summary appraisals of the Nation's ground-water resources --

Arkansas-White-Red Region: U.S. Geological Survey Professional Paper 813-H, $31 \mathrm{p}$.

Bergman, D.L., and Sullivan, C.W., 1963, Channel changes on Sandstone Creek near Cheyenne, Oklahoma, in Geological Survey Research 1963: U.S. Geological Survey Professional Paper 475-C, p. C145-C148.

Clark, W.E., 1963, Evapotranspiration and relation of ground water to surface water in the Pond Creek basin, Oklahoma, Article 221 in Geological Survey Research 1962: U.S. Geological Survey Professional Paper 450-E, p. E142-E145. 
Leonard, A.R., and Ward, P.E., 1962, Use of Na/Cl ratios to distinguish oil field from salt springs brines in western Oklahoma, Article 52 in Geological Survey Research 1962: U.S. Geological Survey Professional Paper 450-B, p. B126-B127.

U.S. Geological Survey, 1954, Water-loss investigations; Lake Hefner studies, technical report: U.S. Geological Survey Professional Paper 269, $158 \mathrm{p}$.

Ward, P.E., 1962, Shallow halite deposits in the Flowerpot Shale in southwestern Oklahoma: U.S. Geological survey Professional Paper 450-E, p. E40-E42.

Ward, P.E., and Leonard, A.R., 1961, Hypothetical circulation of ground water around salt springs in western Oklahoma, Texas, and Kansas, in Geological Survey Research 1961: U.S. Geological Survey, Professional Paper 424-D, p. D150-D151.

\section{U.S. GEOLOGICAI SURVEY WATER-SUPPLY PAPERS}

Bedinger, M.S., Reed, J.E., Wells, C.V., and Swafford, B.F., 1970, Methods and applications of electrical simulation in ground-water studies in the lower Arkansas and Verdigris River valleys, Arkansas and Oklahoma: U.S. Geological Survey Water-Supply Paper 1971, $71 \mathrm{p}$.

Blumer, S.P., 1986, Oklahoma, Surface-water resources, in National water Summary, 1985: U.S. Geological Survey Water-Supply Paper 2300, p. 375-382.

Dover, T.B., Leonard, A.R., and Laine, L.L., 1968, Water for Oklahoma: U.S. Geological Survey Water-Supply Paper 1890 , $107 \mathrm{p}$.

Gould, C.N., 1905, Geology and water resources of Oklahoma: U.S. Geological Survey Water-Supply Paper 148, 178 p., 22 plates.

Havens, J.S., Marcher, M.V., and Schuelein, J.W., 1985, Oklahoma--Ground-water resources, in U.S. Geological Survey, 1985, National Water Summary 1984:

U.S. Geological Survey Water-Supply Paper 2275, $467 \mathrm{p}$.

Kennon, F.W., 1966, Hydrologic effects of small reservoirs in Sandstone Creek watershed, Beckham and Roger Mills Counties, western Oklahoma: U.S. Geological survey Water-Supply Paper 1839-C, $39 \mathrm{p}$. 
Leonard, A.R., 1963, Oklahoma, in The role of ground water in the Nation's water situation: U.S. Geological Survey Water-Supply Paper 1800, p. 671-698.

Marine, I.W., 1963, Correlation of water-level fluctuations with climatic cycles in the Oklahoma Panhandle:

U.S. Geological Survey Water-Supply Paper 1669-K, 10 p.

Renick, B.C., 1925, Additional water supplies for the city of Enid, Oklahoma: U.S. Geological Survey Water-Supply Paper $520-B$, p. $15-26$.

Schwenneson, A.T., 1915a, Ground water for irrigation in the vicinity of Enid, Oklahoma: U.S. Geological Survey Water-Supply Paper 345-B, p. 11-23.

1915b, Ground water for irrigation in the valley of North Fork of Canadian River near Oklahoma City, Oklahoma: U.S. Geological Survey Water-Supply Paper 345-D, p. 41-51.

Tanaka, H.H., 1972, Geohydrology of the lower Verdigris River valley between Muskogee, and Catoosa, Oklahoma:

U.S. Geological Survey Water-Supply Paper 1999-A, 23 p.

Tanaka, H.H., Hollowell, J.R., and Murphy, J.J., 1966, Hydrology of the alluvium of the Arkansas River, Muskogee, Oklahoma, to Fort Smith, Arkansas, with a section on Chemical quality of the water, by J.J. Murphy:

U.S. Geological Survey Water-Supply Paper 1809-T, 42 p.

Thompson, D.C., 1922, Ground water for Irrigation near Gage, Ellis County, Oklahoma: U.S. Geological Survey Water-Supply Paper 500-B, p. 33-53.

U.S. Geological Survey, 1954, Floods of May 1951 in western Oklahoma and northwestern Texas: U.S. Geological Survey Water-Supply Paper 1227-B, p. 135-199.

1984, Oklahoma water issues, in U.S. Geological Survey, 1984, National Water Summary 1983--Hydrologic events and issues, U.S. Geological Survey Water-Supply Paper 2250, $243 \mathrm{p}$.

\section{U.S. GEOLOGICAL SURVEY HYDROLOGIC ATLAS}

Hart, D.L., Jr., 1966, Base of fresh ground water in southern Oklahoma: U.S. Geological Survey Hydrologic Investigations Atlas HA-223, scale 1:250,000, 2 sheets.

Krothe, N.C., Oliver, J.W., and Weeks, J.B., 1982, Dissolved solids and sodium in water from the High Plains aquifer in parts of Colorado, Kansas, Nebraska, New Mexico, Oklahoma, 
South Dakota, Texas, and Wyoming: U.S. Geological Survey Hydrologic Investigations Atlas HA-658, scale $1: 2,500,000$, 2 sheets.

Lohman, S.W., Burtis, V.M., and others, 1953a, Areas of principal ground-water investigations in the Arkansas, White, and Red River basins: U.S. Geological Survey Hydrologic Investigations Atlas HA-2, scale 1:2,500,000, 2 sheets.

1953b, General availability of ground water and depth to water level in the Arkansas, White, and Red River basins: U.S. Geological Survey Hydrologic Investigations Atlas $\mathrm{HA}-3$, scale $1: 2,500,000$.

Luckey, R.R., Gutentag, E.D., and Weeks, J.B., 1981, Water-level and saturated-thickness changes, predevelopment to 1980 , in the High Plains aquifer in parts of Colorado, Kansas, Nebraska, New Mexico, Oklahoma, South Dakota, Texas, and Wyoming: U.S. Geological Survey Hydrologic Investigations Atlas HA-652, scale $1: 2,500,000$, 2 sheets.

Morton, R.B., and Goemaat, R.L., 1972, Reconnaissance of the water resources of Beaver County, Oklahoma:

U.S. Geological Survey Hydrologic Investigations Atlas HA-450, scale $1: 125,000,3$ sheets.

Sapik, D.B., and Goemaat, R.L., 1972, Reconnaissance of the ground-water resources of Cimarron County, Oklahoma: U.S. Geological Survey Hydrologic Investigations Atlas HA-373, scale $1: 125,000,3$ sheets.

Weeks, J.B., and Gutentag, E.D., 1981, Bedrock geology, altitude of base, and 1980 saturated thlckness of the High Plains aquifer in parts of Colorado, Kansas, Nebraska, New Mexico, Oklahoma, South Dakota, Texas, and Wyoming: U.S. Geological Survey Hydrologic Investigations Atlas HA $-648,2$ sheets.

Wood, P.R., and Hart, D.L., Jr., 1967, Avallability of ground water in Texas County, Oklahoma: U.S. Geological Survey Hydrologic Investigations Atlas HA-250, scale $1: 125,000$, 3 sheets. 


\section{U.S. GEOLOGICAL SURVEY MISCELLANEOUS GEOLOGIC INVESTIGATIONS}

Morton, R.B., 1973, Preliminary investigations of the

hydrogeology of the Middle Permian to Tertiary rocks of

the Oklahoma Panhandle: U.S. Geological Survey

Miscellaneous Geologic Investigations Map I-738, scale

approximately $1: 250,000,2$ sheets.

\section{U.S. GEOLOGICAL SURVEY OPEN-FILE REPORTS}

Barclay, J.E., 1951, Ground-water levels in Oklahoma:

U.S. Geological Survey open-file report, 3 p.

Bednar, G.A., and Waldrep, T.E., 1973, Fluvial sediment in Double Creek sub-watershed no. 5, Washington County, Oklahoma: U.S. Geological Survey open-file report, 38 p.

Bergman, D.L., and Huntzinger, T.L., 1981, Rainfall-runoff hydrographs and basin characteristics data for small streams in Oklahoma: U.S. Geological Survey Open-File Report 81-824, $320 \mathrm{p}$.

Bingham, R.H., 1969, Ground-water levels in observation wells in Oklahoma, 1967-68: U.S. Geological Survey open-file report, $92 \mathrm{p}$.

Blumer, S.P., 1983, Sediment data for Mid-Arkansas and Upper Red River basins through 1980: U.S. Geological Survey Open-File Report 83-692, $799 \mathrm{p}$.

Blumer, S.P., and Alf, L.A., 1986, Hydrologic data for selected streams in the coal area of southeastern Oklahoma, July 1978 to September 1982: U.S. Geological Survey Open-File Report 86-319, $337 \mathrm{p}$.

Blumer, S.P., and Hauth, L.D., 1984, Use and availability of continuous streamflow records in Oklahoma:

U.S. Geological Survey Open-File Report 84-747, 23 p.

Blumer, S.P., and Scott, J.C., 1984, Hydrologic data for the Lehigh area, southeastern Oklahoma, May 1977 to January 1982: U.S. Geological Survey Open-File Report 84-599, $212 \mathrm{p}$.

Buckner, H.D., and Kurklin, J.K., 1984, Floods in south-central Oklahoma and north-central Texas: U.S. Geological Survey Open-File Report 84-065, 112 p. (Published by Texas District)

Bohn, J.D., and Hoffman, G.L., 1970, A proposed streamflow data program for Oklahoma: U.S. Geological Survey open-file report, $44 \mathrm{p}$. 
Cady, R.C., 1937 (?), Ground water in Creek County, Oklahoma: U.S. Geological Survey open-file report, $12 \mathrm{p}$.

Carr, J.E., and Havens, J.S., 1976, Records of wells and water quality for the Garber-Wellington aquifer, northern Oklahoma and southern Logan Counties, Oklahoma: U.S. Geological Survey Open-File Report 76-619, 32 p.

Carr, J.E., and Marcher, M.V., 1977, A preliminary appraisal of the Garber-Wellington aquifer, southern Logan and northern Oklahoma Counties, Oklahoma: U.S. Geological Survey Open-File Report 77-278, $23 \mathrm{p}$.

Christenson, S.C., Morton, R.B., Havens, J.S., and Fairchild, R.W., 1988, Geologic logs for selected deep wells in parts of Oklahoma, Texas, and New Mexico: U.S. Geological Survey Open-File Report 86-541, 161 p.

Christenson, S.C., and Parkhurst, D.L., 1987, Ground-water quality assessment of the Central Oklahoma Aquifer, Oklahoma: Project Description: U.S. Geological Survey Open-File Report $87-235,30 \mathrm{p}$.

Corley, R.K., and Huntzinger, T.L., 1979, Flood of August 27-28, 1977, West Cache Creek and Blue Beaver Creek, southwestern Oklahoma: U.S. Geological Survey Open-File Report 79-256, scale $1: 24,000,1$ sheet.

D'Lugosz, J.J., and McClaflin, R.G., 1977, Hydrologic data for the Vamoosa aquifer, east-central Oklahoma:

U.S. Geological Survey Open-File Report 77-487, 38 p. 1978, Geohydrology of the vamoosa aquifer, east-central Oklahoma: U.S. Geological Survey Open-File Report 78-781, $63 \mathrm{p}$.

Davis, L.V., 1955, Ground-water investigations in Oklahoma: U.S. Geological Survey open-file report.

Davis, L.V., and Schoff, S.L., 1948, Ground water in the Blanchard area, MCClain County, Oklahoma: U.S. Geological Survey open-file report, $11 \mathrm{p}$.

Davis, R.E., and Christenson, S.C., 1981, Geohydrology and numerical simulation of the alluvium and terrace aquifer along the Beaver-North Canadian River from the Panhandle to Canton Lake, northwestern Oklahoma, U.S. Geological Survey Open-File Report $81-483,42 \mathrm{p}$.

Davis, R.E., Christenson, S.C., and Blumer, S.P., 1980, Hydrologic data for the alluvium and terrace aquifer along the Beaver-North Canadian River from the Panhandle to 
Canton Lake, northwestern Oklahoma: U.S. Geological Survey Open-File Report 80-159, 77 p.

Davis, R.E., and Hart, D.L., Jr., 1978, Hydrologic data for the Antlers aquifer, southeastern Oklahoma: U.S. Geological Survey Open-File Report 78-1038, 24 p.

Ellis, A.J., 1918, Sources of water supply for the military establishments at Fort Sill, Oklahoma: U.S. Geological Survey open-file report, $11 \mathrm{p}$.

Fader, S.W., and Morton, R.B., 1975a, Ground water in the Verdigris River basin, Kansas and Oklahoma:

U.S. Geological Survey Open-File Report 75-365, 26 p. 1975b, Ground water in the middle Arkansas River basin, Kansas and Oklahoma: U.S. Geological Survey Open-File Report 75-367, $44 \mathrm{p}$.

Fairchild, R.W., 1983, Hydrologic data for Arbuckle Mountain area, south-central Oklahoma: U.S. Geological Survey Open-File Report 83-28, 74 p.

Fairchild, R.W., Hanson, R.L., and Davis, R.E., 1982, Hydrology of the Arbuckle Mountain area: U.S. Geological Survey Open-File Report 82-775, 153 p.

Ferree, D.M., 1983, Ground-water quality data for Oklahoma, 1981, U.S. Geological Survey Open-File Report 83-686, $78 \mathrm{p}$.

1985, Ground-water quality data for Oklahoma--1982-84:

U.S. Geological Survey Open-File Report 85-417, $43 \mathrm{p}$.

Goemaat, R.I., 1976, Ground-water levels in observation wells in Oklahoma, 1971-74: U.S. Geological Survey Open-File Report $76-664,142 \mathrm{p}$.

1977a, Ground-water levels in observation wells in Oklahoma, 1975: U.S. Geological Survey Open-File Report $77-238,35 \mathrm{p}$.

1977b, Selected water-level records for western Oklahoma, 1950-75: U.S. Geological Survey Open-File Report 77-73, $94 \mathrm{p}$.

1977c, Selected water-level records for western Oklahoma, 1975-76: U.S. Geological Survey Open-File Report 77-239, $50 \mathrm{p}$. 
1979, Selected water-level records for Oklahoma, 1976-78:

U.S. Geological Survey Open-File Report 79-1580, 48 p.

Goemaat, R.L., Mize, L.D., Madaj, A.J., and Spiser, D.E., 1986, Ground-water levels in observation wells in Oklahoma, period of record to March 1985: U.S. Geological Survey Open-File Report 86-314, 461 p.

Goemaat, R.L., Mize, D.L., and Spiser, D.E., 1983, Ground-water levels in observation wells in Oklahoma, 1980-82:

U.S. Geological Survey Open-File Report 83-760, 604 p.

1984, Ground-water levels in observation wells in

Oklahoma, 1982-83 climatic years: U.S. Geological Survey Open-File Report 84-472, 583 p.

1985, Ground-water levels in observation wells in Oklahoma, 1983-84 climatic years: U.S. Geological survey Open-File Report 85-87, 588 p.

Goemaat, R.L., and Spiser, D.E., 1978, Selected water-level records for Oklahoma, 1975-77: U.S. Geological Survey Open-File Report 78-721, 58 p.

1979, Selected water-level records for Oklahoma, 1976-78: U.S. Geological Survey Open-File Report 79-1580, 48 p.

Goemaat, R.L., and Willard, C.C., 1983, Ground-water records for the area surrounding the Chickasaw National Recreation Area, Murray County, Oklahoma: U.S. Geological Survey Open-File Report 83-27, 13 p.

Gould, C.N., and Schoff, S.L., 1939, Geological report on water conditions at Platt National Park, Oklahoma: National Park Service Report 249, 38 p., 3 plates. (Also cataloged as U.S. Geological Survey Open-File Report 39-14)

Gutentag, E.D., and Weeks, J.B., 1980, Water table in the High Plains aquifer in 1978 in parts of Colorado, Kansas, Nebraska, New Mexico, Oklahoma, South Dakota, and Wyoming: U.S. Geological Survey Open-File Report 80-50, scale $1: 2,500,000,1$ sheet.

Hanson, R.L., 1984, Droughts: U.S. Geological Survey Open-File Report 84-724 [Chapter in manual for geologic and hydrologic hazards training course.].

Hanson, R.L., Scott, J.C., and Kurklin, J.K., 1983, Oklahoma: A summary of activities of the U.S. Geological Survey, Water Resources Division for 1983: U.S. Geological Survey Open-File Report 83-767, 104 p. 
Hart, D.L., Jr., 1961, Ground water in the alluvium of Beaver Creek basin, Oklahoma: U.S. Geological Survey open-file report, $13 \mathrm{p}$.

1963, Ground-water levels in observation wells in Oklahoma, 1956-60: U.S. Geological Survey open-file report, $196 \mathrm{p}$.

1967, Ground-water levels in observation wells in Oklahoma, 1965-66: U.S. Geological Survey open-file report, $61 \mathrm{p}$.

Hart, D.L., Jr., and Davis, R.E., 1978, Geohydrology of the Antlers area, southeastern Oklahoma: U.S. Geological Survey open-file report, $35 \mathrm{p}$.

Hart, D.L., Jr., Hoffman, G.L., and Goemaat, R.L., 1972, Records of water-level measurements in the Oklahoma Panhandle, 1971-72: U.S. Geological Survey open-file report, $39 \mathrm{p}$.

Hart, D.L., Jr., and others, 1971, Records of water-level measurements in wells in the Oklahoma Panhandle, 1966-70: U.S. Geological Survey open-file report, $66 \mathrm{p}$.

Hauth, L.D., 1985, Floods in central, southwest Oklahoma, October 17-23, 1983: U.S. Geological Survey Open-File Report 85-494, $17 \mathrm{p}$.

Havens, J.S., 1978a, Ground-water records for eastern Oklahoma, Part 2, water-quality records for wells, test-holes, and springs: U.S. Geological Survey Open-File Report 78-357, $139 \mathrm{p}$.

$1978 \mathrm{~b}$, Reconnalssance of ground water in the vicinity of the Wichita Mountains, southwestern Oklahoma:

U.S. Geological Survey Open-File Report 78-857, 27 p. 1985. Hydrologic data: North Canadian River from Lake Overholser to Lake Eufaula, central Oklahoma:

U.S. Geological Survey Open-File Report 84-808, $52 \mathrm{p}$.

Havens, J.S., and Bergman, D.L., 1976a, Ground-water records for northeastern Oklahoma -- Part 1, Records of wells, test holes, and springs: U.S. Geological survey open-file report, $100 \mathrm{p}$.

1976b, Ground-water records for southeastern Oklahoma -Part 1, Records of wells, test holes, and springs:

U.S. Geological Survey open-file report, $59 \mathrm{p}$. 
Hoffman, G.L., and Hart, D.L., Jr., 1973, Records of selected water wells and test holes in the Oklahoma Panhandle:

U.S. Geological Survey open-file report, $59 \mathrm{p}$.

Horak, W.F., and Stoner, J.D., 1988, Oklahoma ground-water quality: U.S. Geological Survey Open-File Report 87-746, 9 p. [Chapter from 1986 National Water Summary]

Hollowell, J.R., 196la, Ground water in the vicinity of Roosevelt, Oklahoma: U.S. Geological Survey open-file report, $8 \mathrm{p}$.

1961b, Ground water in the alluvium of Elk Creek basin, Oklahoma: U.S. Geological Survey open-file report, 20 p.

Huntzinger, T.L., 1978a, High-flow frequencies for selected streams in Oklahoma: U.S. Geological Survey Open-File Report 78-161, $30 \mathrm{p}$.

1978b, Low-flow characteristics of Oklahoma streams: U.S. Geological Survey Open-File Report 78-166, 93 p. 1978c, Application of hydraulic and hydrologic data in urban storm water management: U.S. Geological survey Open-File Report 78-414, $33 \mathrm{p}$.

Kurklin, J.K., 1979, Statistical summaries of surface-waterquality data for selected sites in oklahoma, through the 1975 water year: U.S. Geological Survey Open-File Report $79-219,185 \mathrm{p}$.

Laine, L.L., 1957, Surface-water resources of the Washita River basin in Oklahoma--magnitude, distribution, and quality of streamflow: U.S. Geological survey open-file report, $34 \mathrm{p}$.

1958, Surface waters of North Boggy Creek basin in the Muddy Boggy Creek basin in Oklahoma, with a section on Chemical character of surface water by T.B. Dover: U.S. Geological Survey open-file report, 34 p.

1959a, Surface waters of Little River basin in central Oklahoma, with a section on Chemical character of surface waters by T.B. Dover: U.S. Geological Survey open-file report, $47 \mathrm{p}$.

1959b, Surface waters of Illinois River basin in Arkansas and Oklahoma, with a section on Chemical character of surface waters by T.B. Dover: U.S. Geological Survey open-file report, $65 \mathrm{p}$. 
1962, Surface waters of Cottonwood Creek in the Cimarron River basin in central Oklahoma, with a section on

Chemical quality of surface waters by R.P. Orth:

U.S. Geological Survey open-file report, 41 p.

1963, Surface water of Kiamichi River basin in southeastern Oklahoma, with a section on Quality of water by T.R. Cummings: U.S. Geological Survey open-file report, $39 \mathrm{p}$.

Laine, L.L., and Murphy, J.J., 1962, Surface water of Beaver Creek basin in south-central Oklahoma: U.S. Geological Survey open-file report, $28 \mathrm{p}$.

Leonard, A.R., Davis, L.V., and Stacy, B.L., 1958, Ground water in the alluvial deposits of the Washita River and its tributaries in Oklahoma: U.S. Geological Survey open-file report, $10 \mathrm{p}$.

Marcher, M.V., Bergman, D.L., Slack, L.J., Blumer, S.P., and Goemaat, R.I., 1984, Hydrology of Area 41, Western Region, Interior Coal Province, Oklahoma and Arkansas:

U.S. Geological Survey Water-Resources Investigations Open-File Report 84-129, 86 p.

Marcher, M.V., Kenny, J.F., and others, 1984, Hydrology of Area 40, Western Region, Interior Coal Province, Kansas, Oklahoma, and Missouri: U.S. Geological Survey Water-Resources Investigations Open-File Report 83-266, $97 \mathrm{p}$.

Mize, L.D., 1975, Statistical summaries of streamflow records, Oklahoma, through 1974: U.S. Geological survey open-file report, $399 \mathrm{p}$.

Moore, R.L., 1972, Ground-water levels in observation wells in Oklahoma, 1969-70: U.S. Geological Survey open-file report, $85 \mathrm{p}$.

Morton, R.B., 1980, Digital-model projection of saturated thickness and recoverable water in the ogallala aquifer, Texas County, Oklahoma: U.S. Geological Survey Open-File Report $79-565,34 \mathrm{p}$.

Morton, R.B., and Fader, S.W., 1975, Ground water in the Grand (Neosho) River basin, Kansas and Oklahoma:

U.S. Geological Survey Open-File Report 75-366, 35 p.

Parkhurst, D.L., 1987, Chemical analyses of water samples from the Picher mining area, northeast Oklahoma and southeast Kansas: U.S. Geological Survey Open-File Report 87-453, $43 \mathrm{p}$. 
Playton, S.J., and Davis, R.E., 1977, Preliminary report on the quality of water in abandoned zinc mines in northeastern Oklahoma and southeastern Kansas: U.S. Geological Survey Open-File Report 77-163, $36 \mathrm{p}$.

Schoff, S.L., 1948a, Ground-water conditions in the vicinity of Enid, Oklahoma: U.S. Geological Survey open-file report, $4 \mathrm{p}$.

1948b, Ground-water in the Beggs area, Okmulgee County, Oklahoma: U.S. Geological Survey open-file report, 7 p.

1948c, Ground-water available in the Davenport area [Lincoln County], Oklahoma: U.S. Geological Survey open-file report, $6 \mathrm{p}$.

Schoff, S.L., and Davis, L.V., 1948, Ground water in the Blanchard area, McClain County, Oklahoma: U.S. Geological Survey open-file report.

Slack, L.J., and Blumer, S.P., 1984, Physical and chemical characteristics of water in coal-mine ponds, eastern Oklahoma, June to November 1977-81: U.S. Geological Survey Open-File Report 84-446, 185 p. [Published as Oklahoma Geological Survey Special Publication 87-2.]

Stacy, B.I., 1960, Ground water in the alluvial deposits of Cottonwood Creek basin, Oklahoma: U.S. Geological Survey open-file report, $8 \mathrm{p}$.

1961, Ground-water resources of the alluvial deposits of the Canadian River valley near Norman, Oklahoma:

U.S. Geological Survey open-file report, $61 \mathrm{p}$.

Stoner, J.D., 1977, Index of published surface-water-quality data for Okla- homa, 1946-1975: U.S. Geological Survey Open-File Report 77-204, $212 \mathrm{p}$.

1980, Reconnaissance of polychlorinated biphenyls in the Arkansas River between Muskogee Webbers Falls, Oklahoma: U.S. Geological Survey Open-File Report 80-216, $6 \mathrm{p}$.

Tanaka, H.H., 1960, Maps of parts of Caddo, Custer, and Washita Counties, Oklahoma, showing changes in altitude of water table: U.S. Geological Survey open-file report, 4 sheets.

Tanaka, H.H., Hart, D.I., Jr., and Knott, R.K., 1965, Ground-water data of selected test holes and wells along the Verdigris River in Wagoner and Rogers Counties and along the Arkansas River in Muskogee, Sequoyah, Haskell, and Le Flore Counties, Oklahoma: U.S. Geological Survey open-file report, 4 volumes, $1157 \mathrm{p}$. 
Theis, C.V., 1934, Preliminary geological report on the Salt Plains reservoir site [Alfalfa County], Oklahoma: U.S. Geological Survey open-file report, $15 \mathrm{p}$.

Thomas, W.O., Jr., and Corley, R.K., 1974, Floodflows from small drainage areas in Oklahoma: Progress report and data compilation: U.S. Geological Survey open-file report, $50 \mathrm{p}$.

Tortorelli, R.L., Huntzinger, T.L., Bergman, D.L., and Patneaude, A.L., Jr., 1983, Urban flood analysis in Oklahoma City, Oklahoma: U.S. Geological Survey Open-File Report 83-26, $94 \mathrm{p}$.

Turner, S.F., 1931, Report on water supply for the proposed Southwestern Reformatory at El Reno, Oklahoma: U.S. Geological Survey open-file report, $20 \mathrm{p}$.

U.S. Geological Survey, 1966, Ground water in the Cimarron River basin, New Mexico, Colorado, Kansas, and Oklahoma: U.S. Geological Survey open-file report, 51 p.

1977, Water-resources investigations in Oklahoma, 1976: U.S. Geological Survey open-file report, $6 \mathrm{p}$.

1985, Oklahoma: A summary of activities of the U.S. Geological Survey, Water Resources Division, for 1985: U.S. Geological Survey Open-File Report 85-328, $129 \mathrm{p}$.

Ward, P.E., 1961, Geology and ground-water features of salt springs, seeps, and plains in the Arkansas and Red River basins of western Oklahoma and adjacent parts of Kansas and Texas: U.S. Geological Survey open-file report, 94 p.

Westfall, A.O., 1962a, Surface waters of Elk Creek basin in southwestern Oklahoma: U.S. Geological Survey open-file report, $18 \mathrm{p}$.

1962b, Surface waters of Otter Creek basin in southwestern Oklahoma, with a section on Chemical quality of surface water by J.J. Murphy: U.S. Geological Survey open-file report, $37 \mathrm{p}$.

1963a, Surface water of Little River basin in southeastern Oklahoma, with a section on Quality of water by R.P. Orth: U.S. Geological Survey open-file report, $66 \mathrm{p}$.

1963b, Surface water of Muddy Boggy River basin in south-central Oklahoma, with a section on Quality of water, by T.R. Cummings: U.S. Geological Survey open-file report, $71 \mathrm{p}$. 
Westfall, A.O., and Patterson, J.L., 1964, Floods in Oklahoma, magnitude and frequency: U.S. Geological Survey open-file report, $105 \mathrm{p}$.

Wood, P.R., 1965a, Ground-water levels in observation wells in Oklahoma, 1963-64: U.S. Geological Survey open-file report, $82 \mathrm{p}$.

1965b, Records of ground-water levels and effects of pumping in the Ardmore well-field area, Carter County, Oklahoma: U.S. Geological Survey open-file report, 14 p.

Wood, P.R., and Moeller, M.D., 1964, Ground-water levels in observation wells in Oklahoma, 1961-62: U.S. Geological Survey open-file report, $119 \mathrm{p}$.

\section{U.S. GEOLOGICAL SURVEY WATER-RESOURCES INVESTIGATIONS REPORTS}

Bingham, R.H., Bergman, D.L., and Thomas, W.O., Jr., 1974 , Flood of October 1973 in Enid and vicinity, north-central Oklahoma: U.S. Geological Survey Water-Resources Investigations 27-74, scale 1:250,000, 1:126,720, 2 sheets.

Christenson, S.C., 1983, Numerical simulation of the alluvium and terrace aquifer along the North Canadian River from Canton Lake to Lake Overholser, central Oklahoma: U.S. Geological Survey Water Resources Investigations Report 83-4076, 36 p., 13 plates.

Feder, G.L., and Krothe, N.C., 1981, Results of a reconnaissance water-quality sampling program of the Ogallala aquifer in Colorado, Kansas, Nebraska, Oklahoma, South Dakota, and Texas: U.S. Geological Survey Water-Resources Investigations $81-65,7 \mathrm{p}$.

Hart, D.L., Jr., Hoffman, G.L., and Goemaat, R.L., 1976 , Geohydrology of the Oklahoma Panhandle, Beaver, Cimarron, and Texas Counties: U.S. Geological Survey Water-Resources Investigation 25-75, $62 \mathrm{p}$.

Havens, J.S., 1982a, Altitude and configuration of the 1980 water table in the High Plains regional aquifer, northwestern Oklahoma: U.S. Geological survey Water-Resources Investigations Open-File Report 81-100, scale 1:250,000, 2 sheets.

1982b, Altitude and configuration of the predevelopment water table in the High Plains regional aquifer, northwestern Oklahoma: U.S. Geological Survey Water-Resources Investigations Open-File Report 81-275, scale 1:250,000, 2 sheets. 
19820, Saturated thickness of the High Plains regional aquifer in 1980, northwestern Oklahoma: U.S. Geological Survey Water-Resources Investigations Open-File Report 81-760, scale 1:250,000, 2 sheets.

1982d, Generalized altitude and configuration of the base of the High Plains regional aquifer, northwestern Oklahoma: U.S. Geological Survey Water-Resources Investigations Open-File Report 81-1117, scale 1:250,000, 2 sheets.

1983, Water-level changes in the High Plains regional aquifer, northwestern Oklahoma, predevelopment to 1980: U.S. Geological Survey Water-Resources Investigations Report 83-4073, scale 1:500,000, 1 sheet.

Havens, J.S., and Christenson, S.C., 1983, Numerical simulation of the High Plains regional aquifer, northwestern Oklahoma: U.S. Geological Survey Water-Resources Investigations Report 83-4269, 27 p., 8 plates.

Heimes, F.J., and Luckey, R.R., 1982, Method for estimating historical irrigation requirements from ground water in the High Plains in parts of Colorado, Kansas, Nebraska, New Mexico, Oklahoma, South Dakota, Texas, and Wyoming: U.S. Geological Survey Water-Resources Investigations Report, $82-40,64 \mathrm{p}$.

Krothe, N.C., and Oliver, J.W., 1982, Sulfur isotopic composition and water chemistry in water from the High Plains aquifer, Oklahoma Panhandle and southwestern Kansas: U.S. Geological Survey Water-Resources Investigations $82-12,27 \mathrm{p}$.

Kurklin, J.K., 1985, Water quality in the Blue Creek arm of Lake Eufaula and Blue Creek, Oklahoma, March-October 1978: U.S. Geological Survey Water-Resources Investigations Report 85-4039, $91 \mathrm{p}$.

1986, Water quality in Gaines Creek and Gaines Creek arm of Eufaula Lake, Oklahoma: U.S. Geological Survey Water-Resources Investigations Report 86-4169, $153 \mathrm{p}$. (Being prepared for publication as of 10/02/86)

Marcher, M.V., Bergman, D.L., Slack, L.J., and Blumer, S.P., 1984, Hydrology of Area 41, Western Region, Interior Coal Province, Oklahoma and Arkansas: U.S. Geological Survey Water-Resources Investigations Open-File Report 84-129, $86 \mathrm{p}$. 
Marcher, M.V., Bergman, D.L., Stoner, J.D., and Blumer, S.P., 1981, Preliminary appraisal of the hydrology of the Blocker area, Pittsburg County, Oklahoma: U.S. Geological Survey Water-Resources Investigations Report 81-1187, 48 p., 3 plates.

1983a, Preliminary appraisal of the hydrology of the Rock Island area, Le Flore County, Oklahoma: U.S. Geological Survey Water-Resources Investigations Report 83-4013, 35 p., 3 plates.

1983b, Preliminary appraisal of the hydrology of the Red Oak area, Latimer County, Oklahoma: U.S. Geological Survey Water-Resources Investigations Report 83-4166, 44 p., 3 plates.

Marcher, M.V., Huntzinger, T.L., Stoner, J.D., and Blumer, S.P., 1983, Preliminary appraisal of the hydrology of the Stigler area, Haskell County, Oklahoma: U.S. Geological Survey Water-Resources Investigations Report 82-4099, 37 p., 3 plates.

Marcher, M.V., Kenny, J.F., and others, 1984, Hydrology of Area 40, Western Region, Interior Coal Province, Kansas, Oklahoma, and Missouri: U.S. Geological survey Water-Resources Investigations Open-File Report 83-266, $97 \mathrm{p}$.

Parkhurst, R.S., and Christenson, S.C., 1987, Selected chemical analyses of water from formations of Mesozoic and Paleozoic age in parts of Oklahoma, northern Texas, and Union County, New Mexico: U.S. Geological Survey Water-Resources Investigations Report 86-4355, $222 \mathrm{p}$.

Reed, J.E., 1982, Preliminary projections of the effects of chloride-control structures on the Quaternary aquifer at Great Salt Plains, Oklahoma: U.S. Geological Survey Water-Resources Investigations Report 80-120, $45 \mathrm{p}$.

Sauer, V.B., 1974a, An approach to estimating flood frequency for urban areas in Oklahoma: U.S. Geological Survey Water-Resources Investigations $23-74,10 \mathrm{p}$.

1974b, Flood characteristics of Oklahoma streams: U.S. Geological Survey Water-Resources Investigations $52-73,301 \mathrm{p}$.

Slack, L.J., 1983, Hydrology of an abandoned coal-mining area near McCurtain, Haskell County, Oklahoma: U.S. Geological Survey Water-Resources Investigations Report 83-4202, $117 \mathrm{p}$. 
Stoner, J.D., 1981a, Water type and suitability of Oklahoma surface waters for public supply and irrigation, Part 1;

Arkansas River mainstem and Verdigris, Neosho, and Illinois River basins through 1978: U.S. Geological Survey Water-Resources Investigations 81-33, $297 \mathrm{p}$.

1981b, Water type and suitability of Oklahoma surface waters for public supply and irrigation, Part 2; Salt Fork Arkansas and Cimarron River basins through 1978:

U.S. Geological Survey Water-Resources Investigations $81-39,150 \mathrm{p}$.

1981c, Water type and suitability of Oklahoma surface waters for public supply and irrigation, Part 3; Canadian, North Canadian, and Deep Fork River basins through 1979: U.S. Geological Survey Water-Resources Investigations $81-80,210 \mathrm{p}$.

1982a, Water type and suitability of Oklahoma surface waters for public supply and irrigation, Part 4; Red River mainstem and North Fork Red River through 1979:

U.S. Geological Survey Water-Resources Investiga tions $82-9,235 \mathrm{p}$.

1982b, Water type and suitability of Oklahoma surface waters for public supply and irrigation, Part 5; Washita River basin through 1979: U.S. Geological Survey

Water-Resources Investigations 82-29, $150 \mathrm{p}$.

1984, Estimate of self-supplied domestic water use in Oklahoma during 1980: U.S. Geological Survey

Water-Resources Investigations Report 83-4223, $20 \mathrm{p}$.

1985, Reported withdrawals and estimated use of water in Oklahoma during 1982: U.S. Geological Survey

Water-Resources Investigations Report 85-4084, 96 p.

Thomas, W.O., Jr., 1976, Techniques for estimating flood depths for Oklahoma streams: U.S. Geological Survey

Water-Resources Investigations 2-76, $170 \mathrm{p}$.

Thomas, W.O., Jr., and Corley, R.K., 1973, 1971-72 Floods of Glover Creek and Little River in southeastern Oklahoma: U.S. Geological Survey Water-Resources Investigations 5-73, scale 1:24,000, 2 sheets.

1977, Techniques for estimating flood discharges for Oklahoma streams: U.S. Geological Survey Water-Resources Investigations 77-54, $170 \mathrm{p}$. 
Tortorelli, R.L., and Bergman, D.L., 1984, Techniques for estimating flood peak discharges for unregulated streams and streams regulated by small floodwater retarding structures in Oklahoma: U.S. Geological Survey Water-Resources Investigations Report 84-4358, $85 \mathrm{p}$.

Weeks, J.B., 1978, Plan of study for the High Plains regional aquifer systems analysis in parts of Colorado, Kansas, New Mexico, Oklahoma, South Dakota, Texas, and Wyoming: U.S. Geological Survey Water-Resources Investigations $78-70,28 \mathrm{p}$.

\section{U.S. GEOLOGICAL SURVEY FEMA FLOOD REPORTS}

Bergman, D.L., 1980a, City of Sallisaw, Oklahoma: Federal Emergency Management Agency Flood Insurance Study, community number 400199, Washington D.C., 23 p.

1980b, City of Edmond, Oklahoma: Federal Emergency Management Agency Flood Insurance Study, community number 400252, Washington D.C., 41 p.

1980c, City of the Village, Oklahoma: Federal Emergency Management Agency Flood Insurance Study, community number 400420, Washingt on D.C., 15 p.

1982, City of Nichols Hills, Oklahoma: Federal Emergency Management Agency Flood Insurance Study, community number 400423, Washington D.C., $15 \mathrm{p}$.

Bergman, D.L., and Walton, C., 1979, City of Duncan, Oklahoma: Federal Emergency Management Agency Flood Insurance Study, community number 400202, Washington D.C., 24 p.

Corley, R.K., 1980, City of Ponca City, Oklahoma: Federal Emergency Management Agency Flood Insurance Study, community number 400080, Washington D.C., 23 p.

Huntzinger, T.L., 1979a, City of Enid, Oklahoma: Federal Emergency Management Agency Flood Insurance Study, community number 400062, Washington D.C., 31 p.

1979b, City of McAlester, Oklahoma: Federal Emergency Management Agency Flood Insurance Study, community number 400170, Washington D.C., 22 p.

1980a, City of Mustang, Oklahoma: Federal Emergency Management Agency Flood Insurance Study, community number 400409, Washington D.C., 15 p. 
1980b, Town of Nicoma Park, Oklahoma: Federal Emergency Management Agency Flood Insurance Study, community number 400424, Washington D.C., 15 p.

1981a, Town of North Enid, Oklahoma: Federal Emergency Management Agency Flood Insurance Study, community number 400425, Washington D.C., $14 \mathrm{p}$.

1981b, City of Choctaw, Oklahoma: Federal Emergency Management Agency Flood Insurance Study, community number 400357, Washington D.C., $23 \mathrm{p}$.

1981c, City of Midwest City, Oklahoma: Federal Emergency Management Agency Flood Insurance Study, community number 400405, Washington D.C., 21 p.

Huntzinger, T.L., and Tortorelli, R.L., 1980a, City of Spencer, Oklahoma: Federal Emergency Management Agency Flood Insurance study, community number 400412 , Washington D.C., $15 \mathrm{p}$.

1980b, City of Warr Acres, Oklahoma: Federal Emergency Management Agency Flood Insurance Study, community number 400449, Washington D.C., 13 p.

1981a, Town of Jones, Oklahoma: Federal Emergency Management Agency Flood Insurance Study, community number 400141, Washington D.C., $20 \mathrm{p}$.

1981b, Town of Valley Brook, Oklahoma: Federal Emergency Management Agency Flood Insurance Study, community number 400445, Washington D.C., 13 p.

Huntzinger, T.L., Tortorel1i, R.L., and Bergman, D.L., 1982 , City of Oklahoma City, Oklahoma: Federal Emergency Management Agency Flood Insurance Study, community number 405378, Washington, D.C., 194 p.

Tortorelli, R.L., 1982, Town of Piedmont, Oklahoma: Federal Emergency Management Agency Flood Insurance Study, community number 400027, Washington, D.C., 17 p.

1986, Limited detail flood insurance study, City of Tishomingo, Johnston County, Oklahoma: Federal Emergency Management Agency Flood Insurance Study, community number 400077, Washington, D.C., 9 p.

Tortorelli, R.L., Huntzinger, T.L., and Bergman, D.L., 1982, Oklahoma County, Oklahoma: Federal Emergency Management Agency Flood Insurance Study, community number 400466 , Washington, D.C., $21 \mathrm{p}$. 


\section{U.S. GEOLOGICAL SURVEY WATER-RESOURCES DATA REPORTS}

(ANNUAL REPORTS)

Hauth, L.D., Kurklin, J.K., and Walters, D.M., 1985, Water resources data for Oklahoma, water year 1983:

U.S. Geological Survey Water-Data Report OK-83-1, 286 p.

1986, Water resources data, Oklahoma, water year 1984:

U.S. Geological Survey Water-Data Report OK-84-1, 302 p.

Hauth, L.D., Kurklin, J.K., Walters, D.M., and Ferree, D.M., 1984, Water resources data, Oklahoma, water year 1982: U.S. Geological Survey Water-Data Report OK-82-1, 336 p.

U.S. Geological Survey, 1961-64, Surface-water records of Oklahoma. (One volume for each year).

1964, Quality-of-water records of Oklahoma.

1965-74, Water resources data for Oklahoma -- Part 1 , Surface-water records; Part 2, Water-quality records. (One volume of each for each year).

1976, Water resources data for Oklahoma, water year 1975:

U.S. Geological Survey Water-Data Report OK-75-1, 528 p.

1977a, Water resources data for Oklahoma, water year 1976 , Volume 1, Arkansas River basin: U.S. Geological Survey

Water-Data Report OK-76-1, $513 \mathrm{p}$.

1977b, Water resources data for Oklahoma, water year 1976 , Volume 2, Red River basin: U.S. Geological Survey

Water-Data Report OK-76-2, $216 \mathrm{p}$.

1978a, Water resources data for Oklahoma, water year 1977 , Volume 1, Arkansas River basin: U.S. Geolog1cal Survey

Water-Data Report OK-77-1, $542 \mathrm{p}$.

1978b, Water resources data for Oklahoma, water year 1977 , Volume 2, Red River basin: U.S. Geological Survey

Water-Data Report OK-77-2, $235 \mathrm{p}$.

1979a, Water resources data for Oklahoma, water year 1978, Volume 1, Arkansas River basin: U.S. Geological Survey

Water-Data Report OK-78-1, $523 \mathrm{p}$.

1979b, Water resources data for Oklahoma, water year 1978, Volume 2, Red River basin: U.S. Geological Survey

Water-Data Report OK-78-2, $249 \mathrm{p}$. 
1981a, Water resources data for Oklahoma, water year 1979, Volume 1, Arkansas River basin: U.S. Geological Survey

Water-Data Report OK-79-1, $629 \mathrm{p}$.

1981b, Water resources data for Oklahoma, water year 1979, Volume 2, Red River basin: U.S. Geological Survey

Water-Data Report OK-79-2, $249 \mathrm{p}$.

1982, Water resources data for Oklahoma, water year 1980, Volume 1: U.S. Geological Survey Water-Data Report

OK-80-1, $613 \mathrm{p}$.

1983, Water resources data - Oklahoma, water year 1981:

U.S. Geological Survey Water-Data Report OK-81-1, 546 p.

1984, Water resources data - Oklahoma, water year 1982:

U.S. Geological Survey Water-Data Report OK-82-1, 336 p.

\section{U.S. GEOLOGICAL SURVEY CIRCULAR}

Irwin, J.H., and Morton, R.B., 1969, Hydrogeologic information on the Glorieta Sandstone and Ogallala Formation in the Oklahoma Panhandle and adjoining areas as related to underground waste disposal: U.S. Geological survey Circular 630, $26 \mathrm{p}$.

\section{U.S. GEOLOGICAL SURVEY ANNUAL REPORTS}

Johnson, W.D., 1901, The High Plains and their utilization:

U.S. Geological Survey Twenty-first Annual Report, IV,

p. 601-741.

1902, The High Plains and their utilization: U.S. Geological Survey Twenty-second Annual Report, IV, p. 631-639.

\section{U.S. GEOLOGICAL SURVEY MISCELLANEOUS REPORTS}

Miser, H.D., 1854, Geologic map of Oklahoma: Oklahoma Geological Survey and U.S. Geological Survey, scale 1:500,000.

U.S. Geological Survey, 1976, Hydrologic Unit Map of Oklahoma - 1974: U.S. Geological Survey, scale 1:500,000,

1 sheet.

OKLAHOMA WATER RESOURCES BOARD

Barclay, J.E., and Burton, L.C., 1953, Ground-water resources of the terrace deposits and alluvium of western Tillman County, Oklahoma: Oklahoma Planning and Resources Board Division of Water Resources Bulletin 12, 71 p. 
Burton, L.C., 1965, Ground water in terrace deposits of central Beckham County, Oklahoma: Oklahoma Water Resources Board Bulletin 25, $30 \mathrm{p}$.

Cummings, T.R., 1963, Chemical character of surface waters in Oklahoma 1957-58: Oklahoma Water Resources Board Bulletin $19,165 \mathrm{p}$.

1964, Chemical character of surface waters in Oklahoma 1958-59: Oklahoma Water Resources Board Bulletin 20, $133 \mathrm{p}$.

1965a, Chemical character of surface waters in Oklahoma 1960-61: Oklahoma Water Resources Board Bulletin 23, $178 \mathrm{p}$.

1965b, Chemical character of surface waters in Oklahoma 1961-62: Oklahoma Water Resources Board Bulletin 24, $203 \mathrm{p}$.

1966a, Chemical character of surface waters in Oklahoma 1959-60: Oklahoma Water Resources Board Bulletin 22, $167 \mathrm{p}$.

1966b, Chemical character of surface waters in Oklahoma 1962-63: Oklahoma Water Resources Board Bulletin 30 , $200 \mathrm{p}$.

Dover, T.B., 1953a, Chemical character of surface waters in Oklahoma 1950-51: Oklahoma Planning and Resources Board Division of Water Resources Bulletin 7, $88 \mathrm{p}$.

1953b, Chemical character of public water supplies of Oklahoma 1953: Oklahoma Planning and Resources Board Division of Water Resources Bulletin 8, $47 \mathrm{p}$.

1954, Chemical character of surface waters of Oklahoma 1951-52: Oklahoma Planning and Resources Board Division of Water Resources Bulletin 10, $115 \mathrm{p}$.

1956, Chemical character of surface waters of Oklahoma 1953-54: Oklahoma Planning and Resources Board Division of Water Resources Bulletin 14, $141 \mathrm{p}$.

1958, Chemical character of surface waters of Oklahoma 1954-55: Oklahoma Water Resources Board Bulletin 15, $117 \mathrm{p}$.

1959, Chemical character of surface waters of Oklahoma 1955-56: Oklahoma Water Resources Board Bulletin 16, $144 \mathrm{p}$. 
Dover, T.B., and Murphy, J.J., 1955, A reconnaissance of the chemical and physical quality of Pryor Creek in the vicinity of Pryor, Oklahoma: Oklahoma Planning and Resources Board Division of Water Resources Bulletin 13, $32 \mathrm{p}$.

Gilbert, C.R., 1959, Hydrologic and physical data for Sandstone Creek watershed in western Oklahoma 1951-56: Oklahoma Water Resources Board Bulletin 17, $124 \mathrm{p}$.

Hart, D.L., Jr., 1965, Ground water in the alluvial deposits of the Washita River between Clinton and Anadarko, Oklahoma: Oklahoma Water Resources Board Bulletin 26, 23 p.

Hollowell, J.R., 1965a, Ground water in the alluvium of Otter Creek basin, Oklahoma: Oklahoma Water Resources Board Bulletin 27, $15 \mathrm{p}$.

1965b, Ground water in the alluvium of Elk Creek basin, Oklahoma: Oklahoma Water Resources Board Bulletin 28, $12 \mathrm{p}$.

Laine, L.L., 1940(?), Provisional report on flood of September 1940, and miscellaneous discharge measurements, in Dam failure at Cleveland caused by storm of September 4, 1940: Oklahoma Planning and Resources Board Division of Water Resources, p. 7-18.

Laine, L.L., Schoff, S.L., and Dover, T.B., 1951, Public water supplies in Oklahoma: Oklahoma Planning and Resources Board Division of Water Resources, $110 \mathrm{p}$.

Leonard, A.R., 1960, Ground water in Oklahoma: Oklahoma Water Resources Board, $12 \mathrm{p}$.

Murphy, J.J., 1955, Chemical character of surface waters of Oklahoma 1952-53: Oklahoma Planning and Resources Board Division of Water Resources Bulletin 11, $128 \mathrm{p}$.

Pate, C.O., Murphy, J.J., and Orth, R.P., 1961, Chemical character of surface waters of Oklahoma 1956-57: Oklahoma Water Resources Board Bulletin 18, $138 \mathrm{p}$.

Reed, E.W., Mogg, J.L., Barclay, J.E., and Peden, G.H., 1952, Ground-water resources of the terrace deposits along the northeast side of the Cimarron River in Alfalfa, Garfield, Kingfisher, and Major Counties, Oklahoma: Oklahoma Planning and Resources Board Division of Water Resources Bulletin 9, $101 \mathrm{p}$. 
Reed, E.W., Oakland, G.L., and Jacobsen, C.L., 1945, Oklahoma water: Oklahoma Planning and Resources Board Division of Water Resources, $145 \mathrm{p}$.

Steele, C.E., and Barclay, J.E., 1965, Ground-water resources of Harmon County and adjacent parts of Greer and Jackson Counties, Oklahoma: Oklahoma Water Resources Board Bulletin 29, $96 \mathrm{p}$.

Walling, I.W., 1949, Chemical character of surface waters in the Washita River basin of Oklahoma, 1946-47: Oklahoma Planning and Resources Board Division of Water Resources Bulletin 4, $31 \mathrm{p}$.

1952, Chemical character of surface waters in Oklahoma 1949-50: Oklahoma Planning and Resouroes Board Division of Water Resources Bulletin 6,70 p.

Walling, I.W., Schoff, S.L., and Dover, T.B., 1951, Chemical character of surface waters in Oklahoma 1946-49: Oklahoma Planning and Resources Board Division of Water Resources Bulletin 5, $180 \mathrm{p}$.

Wood, P.R., and Stacy, B.L., 1965, Geology and ground-water resources of Woodward County, Oklahoma: Oklahoma Water Resources Board Bulletin 21, 114 p.

\section{OKLAHOMA WATER RESOURCES BOARD HYDROLOGIC INVESTIGATIONS}

Wickersham, Ginia, 1979, Ground water resources of the southern part of the Garber-Wellington ground water basin, in Cleveland and southern Oklahoma Counties and parts of Pottawatomie County, Oklahoma: Oklahoma Water Resources Board Hydrologic Investigations, Publication 86,3 sheets.

\section{OKLAHOMA GEOLOGICAL SURVEY BULLETIN}

Bingham, R.H., 1979, Water Resources [of Noble County, Oklahoma], in Shelton, John W., 1979, Geology and mineral resources of Noble County, Oklahoma: Oklahoma Geological Survey Bulletin 128, $66 \mathrm{p}$.

Davis, L.V., 1955, Geology and water resources of the Grady and northern Stephens Counties, Oklahoma: Oklahoma Geological Survey Bulletin 73, $184 \mathrm{p}$.

1960, Geology and ground-water resources of southern McCurtain County, Oklahoma: Oklahoma Geological Survey Bulletin 86, $108 \mathrm{p}$. 
Hart, D.L., Jr., 1978, Ground water in Custer County, in Fay, Robert 0., 1978, Geology and mineral resources (exclusive of petroleum) of Custer County, Oklahoma: Oklahoma Geological Survey Bulletin 114, $88 \mathrm{p}$.

Marine, I.W., and Schoff, S.L., 1962, Ground-water resources of Beaver County, Oklahoma: Oklahoma Geological Survey Bulletin $97,74 \mathrm{p}$.

Mogg, J.L., Schoff, S.L., and Reed, E.W., 1960, Ground water resources of Canadian County, Oklahoma: Oklahoma Geological Survey Bulletin 7, $112 \mathrm{p}$.

Motts, W.S., 1963, Water resources of Okmulgee County, part 2 of Oklahoma Geological Survey, Geology and water resources of Okmulgee County, Oklahoma: Oklahoma Geological Survey Bulletin 91, p. 5-6, 81-123.

Schoff, S.L., 1939, Geology and ground-water resources of Texas County, Oklahoma: Oklahoma Geological survey Bulletin 59, $248 \mathrm{p}$.

1943, Geology and ground-water resources of Cimarron County, Oklahoma, with a section on Mesozoic stratigraphy by J.W. Stovall: Oklahoma Geological Survey Bulletin 64, $317 \mathrm{p}$.

Schoff, S.L., Reed, E.W., and Branson, C.C., 1955, Geology and ground-water resources of Ottawa County, Oklahoma:

Oklahoma Geological Survey Bulletin 72, 203 p.

Warren, J.H., 1952, Water resources of Tulsa County, in Geology and mineral resources of Tulsa County, Oklahoma: Oklahoma Geological Survey Bulletin 69, p. 140-155.

\section{OKLAHOMA GEOLOGICAL SURVEY CIRCULARS}

D'Lugosz, J.J., McClaflin, R.G., and Marcher, M.V., 1986, Geohydrology of the Vamoosa-Ada aquifer, east-central Oklahoma: Oklahoma Geological Survey Circular 87, 42 p., $3 \mathrm{pl}$.

Havens, J.S., 1983, Reconnaissance of ground water in the vicinity of the Wichita Mountains, southwestern Oklahoma: Oklahoma Geological Survey Circular 85, 13 p.

Morton, R.B., 1986, Effects of brine on the chemical quality of water in parts of Creek, Lincoln, Okfuskee, Payne, Pottawatomie, and Seminole Counties, Oklahoma: Oklahoma Geological Survey Circular 89, 38 p., 1 plate. 
Playton, S.J., Davis, R.E., and MoClaflin, R.G., 1980, Chemical quality of water in abandoned zino mines in northeastern Oklahoma and southeastern Kansas: Oklahoma Geological Survey Circular $82,49 \mathrm{p}$. (Also released as USGS OFR 78-294.)

Schoff, S.L., and Reed, E.W., 1951a, Ground-water resources of the Arkansas River flood plain near Fort Gibson, Muskogee County, Oklahoma: Oklahoma Geological Survey Circular 28, $55 \mathrm{p}$.

Tanaka, H.H., and Davis, L.V., 1963, Ground-water resources of the Rush Springs Sandstone in the Caddo County area, Oklahoma: Oklahoma Geological Survey Ciroular 61, 63 p.

Wood, P.R., and Burton, L.C., 1968, Ground-water resources of Cleveland and Oklahoma Counties, Oklahoma: Oklahoma Geological Survey Circular 71, 75 p.

OKLAHOMA GEOLOGICAL SURVEY MINERAL REPORTS

Davis, L.V., 1950, Ground water in the Pond Creek basin, Caddo County, Oklahoma: Oklahoma Geological Survey Mineral Report 22, $23 \mathrm{p}$.

Dott, R.H., 1942, Geology of Oklahoma ground-water supplies: Oklahoma Geological Survey Mineral Report 11, 26 p.

Jacobsen, C.L., and Reed, E.W., 1949, Ground-water supplies in the Oklahoma City area, Oklahoma: Oklahoma Geological Survey Mineral Report 20, $25 \mathrm{p}$.

Schoff, S.L., 1948, Ground-water irrigation in the Duke area, Jackson and Greer Counties, Oklahoma: Oklahoma Geological Survey Mineral Report 18, $8 \mathrm{p}$.

1949, Ground-water in Kingfisher County, Oklahoma: Oklahoma Geological Survey Mineral Report 19, 20 p. 1950, Ground-water in the Cherokee area, Alfalfa County, Oklahoma: Oklahoma Geological Survey Mineral Report 21, $17 \mathrm{p}$.

OKIAHOMA GEOLOGICAL SURVEY GEOLOGIC MAP

Schoff, S.L., 1955, Map of ground-water reservoirs in Oklahoma: Oklahoma Geological Survey Map GM-2, scale 1:750,000, 1 sheet. 


\section{ORLAHOMA GEOLOGICAL SURVEY EDUCATIONAL PUBLICATION}

Marcher, M.V., 1972, Major sources of water in Oklahoma, in Johnson, K.S., and others, 1972, Geology and earth resources of Oklahoma, an atlas of maps and oross sections: Oklahoma Geological Survey Educational Publication 1, p. 8 .

\section{OKLAHOMA GEOLOGICAL SURVEY HYDROLOGIC ATLAS}

Bingham, R.H., and Bergman, D.L., 1980, Reconnalssance of the water resources of the Enid quadrangle, north-central Oklahoma: Oklahoma Geological Survey Hydrologic Atlas HA-7, 4 sheets, scale $1: 250,000$.

Bingham, R.H., and Moore, R.L., 1975, Reconnaissance of the water resources of the Oklahoma City quadrangle, central Oklahoma: Oklahoma Geological Survey Hydrologic Atlas 4, scale $1: 250,000,4$ sheets.

Carr, J.E., and Bergman, D.L., 1976, Reconnaissance of the water resources of the clinton quadrangle, west-central Oklahoma: Oklahoma Geological Survey Hydrologic Atlas 5, scale $1: 250,000,4$ sheets.

Hart, D.L., Jr., 1974, Reconnaissance of the water resources of the Ardmore and Sherman quadrangles, southern Oklahoma: Oklahoma Geological Survey Hydrologic Atlas 3, scale $1: 250,000,4$ sheets.

Havens, J.S., 1977, Reconnaissance of the water resources of the Lawton quadrangle, southwestern Oklahoma: Oklahoma Geological Survey Hydrologic Atlas 6, scale 1:250,000, 4 sheets.

Marcher, M.V., 1969, Reconnaissance of the water resources of the Fort Smith quadrangle, east-central Oklahoma:

Oklahoma Geological Survey Hydrologic Atlas 1, scale $1: 250,000,4$ sheets.

Marcher, M.V., and Bergman, D.L., 1983, Reconnaissance of the water resources of the MoAlester and Texarkana quadrangles, southeastern Oklahoma: Oklahoma Geological Survey Hydrologic Atlas 9, scale 1:250,000, 4 sheets.

Marcher, M.V., and Bingham, R.H., 1971, Reconnaissance of the water resources of the Tulsa quadrangle, northeastern Oklahoma: Oklahoma Geological Survey Hydrologic Atlas 2, scale $1: 250,000,4$ sheets. 
Morton, R.B., 1980b, Reconnaissance of the water resources of the Woodward quadrangle, northwest Oklahoma: Oklahoma Geological Survey Hydrologic Atlas 8, scale 1:250,000, 4 sheets.

\section{ORLAHOMA GEOLOGICAL SURVEY HOPPER AND GEOLOGY NOTES}

Reed, E.W., 1949, Unusual fluctuations in Rush Springs wells: Oklahoma Geological Survey, The Hopper, v. 9, no. 7, p. $69-70$.

1950. Increased use of ground water for irrigation in the Duke area, Oklahoma: Oklahoma Geological Survey, The Hopper, v. 10, p. 86-90.

Reed, E.W., and Schoff, S.L., 1947, Ground-water storage increases in Tillman County, Oklahoma: Oklahoma

Geological Survey, The Hopper, v. 7, p. 77-80.

Schoff, S.L., 1942, Geology and ground-water resources of Beaver County, Oklahoma: Oklahoma Geological Survey, The Hopper, v. 2, no. 10, p. 94-97.

1948, Ground-water at high stage in Oklahoma Panhandle: Oklahoma Geological Survey, The Hopper, v. 8, no. 8, p. 74-76.

1950, Deep well irrigation in Oklahoma Panhandle: Oklahoma Geological Survey, The Hopper, v. 10, no. 8, p. 76 .

1953, Ground-water pumpage and water levels in Oklahoma: Oklahoma Geological Survey, The Hopper, v. 13, no. 9-12, p. 51-57.

Bingham, R.H., 1969a, Springs in northeastern Oklahoma: Oklahoma Geological survey Oklahoma Geology Notes, v. 29, no. 1, p. 19 (Abstract).

1969b, Springs in the Ozark Region, northeastern Oklahoma: Oklahoma Geological Survey Oklahoma Geology Notes, v. 29, no. 6, p. $135-145$.

Blumer, S.P., and Slack, L.J., 1986, Physical and chemical characteristics of water in coal-mine ponds, eastern Oklahoma: Oklahoma Geological Survey Oklahoma Geology Notes, v. 46, no.4, p. 128-134.

Davis, L.V., 1958a, Ground water in the Arbuckle and Simpson Groups in the Arbuckle Mountains, Oklahoma: Oklahoma Geological Survey Oklahoma Geology Notes, v. 18, no. 10, p. 152-157. 
1958b, Oklahoma's underground water: Oklahoma Geological Survey Oklahoma Geology Notes, v. 18, no. 12, p. 189-202.

Fairchild, R.W., 1984, Springs in the Arbuckle Mountain area, south-central Oklahoma: Oklahoma Geological Survey Oklahoma Geology Notes, v. 44, no. 1, p. 4-11.

Hart, D.L., Jr., 1961, Fluctuations of water levels in wells: Oklahoma Geological Survey Oklahoma Geology Notes, v. 21 , no. 2, p. $41-47$.

Hauth, L.D., 1985, An overview of hydrologic-data collection by the U.S. Geological Survey in Oklahoma: Oklahoma Geological Survey Oklahoma Geology Notes, v. 45, no. 4, p. 149-161.

Havens, J.S., 1985, Water-level changes in the Ogallala aquifer, northwestern Oklahoma: Oklahoma Geological Survey Oklahoma Geology Notes, v. 45, no. 5, p. 205-210.

Scott, J.C., 1984, Use of minicomputers in water-resources investigations: Oklahoma Geological Survey Oklahoma Geology Notes, v. 44, no. 6, p. 188-89.

Tanaka, H.H., 1958, Changes in ground-water levels in Oklahoma during 1957: Oklahoma Geological Survey Oklahoma Geology Notes, v. 18 , no. $3, \mathrm{p} .57$.

U.S. Geological Survey, 1974, Summary of October 1973 rainstorm, Enid and vicinity, north-central oklahoma: Oklahoma Geological survey Oklahoma Geology Notes, v. 34 , no. 6, p. 209-212.

Ward, P.E., 196la, Salt springs in Oklahoma: Oklahoma Geological Survey Oklahoma Geology Notes, v. 21, no. 3, p. 81-84.

1961b, Shallow halite deposits in northern Woodward and southern Woods Counties, Oklahoma: Oklahoma Geological Survey Oklahoma Geology Notes, v. 21, no. 10, p. 275-277.

\section{OKLAHOMA GEOLOGICAL SURVEY SPECIAL PUBLICATIONS}

Slack, L.J., and Blumer, S.P., 1987, Physical and chemical characteristics of water in coal-mine ponds, eastern Oklahoma: Oklahoma Geological Survey Special Publication 87-2, $116 \mathrm{p}$. 
Dover, T.B., Horton, John, and Leonard, A.R., 1957, A look at the water resources of Oklahoma: Oklahoma City Geological Society Shale Shaker, v. 7, no. 10, p. 18-22, 24-32.

Fairchild, R.W., and Christenson, S.C., 1982, Potential contamination of the Roubidoux aquifer by water from abandoned zinc mines, northeastern Oklahoma: Abstract in Geological Society of America Abstracts with Programs, v. 13 , no. 3 .

Fairchild, R.W., and Davis, R.E., 1978, Structural control of ground-water flow in the Arbuckle Mountain area, south-central Oklahoma: Abstract in Geological Society of America Abstracts with Programs, v. 10, no. 1, p. 5.

Falrchild, R.W., Davis, R.E., and Hanson, R.L., 1979, Aquifer characteristics of the Arbuckle aquifer, south-central Oklahoma: Abstract in Proceedings of the Geological Society of America, South-Central Section, Mountain View, Arkansas, April 9-10, 1979, v. 11, no. 2, p. 147.

Frye, J.C., and Schoff, S.L., 1942, Deep-seated solution in the Meade basin and vicinity, Kansas and Oklahoma: American Geophysical Union Transactions, v. 24, pt. 1, p. 35-39.

Hart, D.L., Jr., and Davis, R.E., 1979, Geohydrology of the Antlers aquifer, southeastern Oklahoma: Abstract in Proceedings of the Geological Society of America, South-Central Section, Mountain View, Arkansas, April $9-10,1979$, v. 11 , no. 2, p. 148.

Irwin, J.H., 1971, Ground-water investigations in Oklahoma, in Environmental Aspects of Geology and Engineering in Oklahoma, Annals of the Oklahoma Academy of Science Publication No. 2: Oklahoma Geological Survey, p. 58.

1977. Water resources of Oklahoma, in Geography of Oklahoma, edited by John W. Morris: Oklahoma Historical Society, Oklahoma City, Oklahoma, p. 25-39.

Irwin, J.H., and Morton, R.B., 1970, Hydrogeologic information on the Ogallala Formation in the Oklahoma Panhandle and adjoining areas as related to underground waste disposal: Abstract in Symposium on the Ogallala Aquifer, Lubbock, Texas: Texas Technological University, p. 30.

Moench, A.F., Sauer, V.B., and Jennings, M.E., 1974, Modification of routed streamflow by channel loss and base flow: American Geophysical Union, Water Resources Research, v. 10, p. 963-968. 
Muller, A.B., Parkhurst, D.L., and Tasker, P.W., 1986, Use of the PHREEQE code in modelling environmental geochemical problems encountered in performance assessment modelling, in Symposium on ground-water flow and transport modelling for performance assessment of deep geologic disposal of radioactive waste--A critical evaluation of the state of the art: Sponsored by U.S. Department of Energy, Civilian Waste Management, May 20-21, 1985, Albuquerque, New Mexico.

Playton, S.J., Davis, R.E., and McClaflin, R.G., 1978, Water quality in abandoned zinc mines in the Picher field, Tri-State mining district: Abstract in Geological Society of America Abstracts with Programs, v. 10, no. 1, p. 24.

Reed, E.W., and Schoff, S.L., 1952, Aquifers in Ottawa County, Oklahoma: Oklahoma Academy of Science Proceedings, v. 33 , p. 194-195.

Sauer, V.B., 1973, Unit response method of open-channel flow routing: American Society of Civil Engineers Proceedings, Journal of the Hydraulios Division, v. 99, p. 179-193.

Schoff, S.L., 1948, Ground-water supplies and uses in Oklahoma, in Report of Proceedings, Oklahoma Conservation Conferences, 3d: Stiliwater, Oklahoma Research Foundation, Oklahoma Agricultural and Mechanical College, p. 24-36.

1955, Triassic rocks on Goff Creek, Texas County, Oklahoma: Oklahoma Academy of Science, v. XXXLV, p. 149-152.

Schoff, S.I., and Reed, E.W., 1951, Ground water in alluvial deposits in Oklahoma: Economic Geology, v. 46, no. 1, p. $76-83$.

Smith, O.M., 1942, The chemical analysis of the waters of Oklahoma, with contributions by R.H. Dott and E.C. Warkentin: Oklahoma Agricultural and Mechanical College, Engineering Experiment Station Publication no. $52,474 \mathrm{p}$.

\section{MISCELLANEOUS}

Gould, C.N., and Schoff, S.I., 1939, Geological report on water conditions at Platt National Park, Oklahoma: National Park Service Report 249, 38 p., 3 plates. (Also cataloged as U.S. Geological Survey Open-File Report 39-14) 
Irwin, J.H., 1971, The Glorieta Sandstone and the Ogallala Formation as related to underground disposal in the High Plains: Arkansas-White-Red River Basins Interagency Committee, January 1971, Minutes, Tulsa, Oklahoma, appendix X, p. 2-15.

Oklahoma Water Resources Board, 1975, Salt water detection in the Cimarron terrace, Oklahoma: U.S. Environmental Protection Agency Ecological Research Series EPA-660/3-74-033, $166 \mathrm{p}$. 


\section{SOURCES OF O.S. GEOLOGICAL SURVBY}

PUBLICATIONS AND BOOKS

BOOKS, MAPS, AND PERIODICALS

Since 1879, the U.S. Geological Survey has served the public, and Federal, State, and local governments by collecting, analyzing, and publishing detailed information about the Nation's mineral, land, and water resources. This information is in a variety of map, book, and other formats, and is available from several sources within the Geological Survey.

Text Products - - Books and Open-File Reports

To order USGS book publications, catalogs, and pamphlets, and for information on the availability of microfiche or paper duplicate copies of selected open-file reports, write:

Books and Open-File Reports Section

U.S. Geological Survey

Federal Center, Box 25425

Denver, CO 80225

(303)236-7476

Maps

To order geologic, hydrologic, topographic, and land-use and land-cover maps published by the USGS, write:

Map Distribution Section

U.S. Geological Survey

Federal Center, Box 25286

Denver, CO 80225

(303) $236-7477$

New Publications

To get on the mailing list for the free monthly catalog

"New Publications of the Geological Survey" write:

U.S. Geological Survey

582 National Center

Reston, VA 22092

\section{INFORMATION}

\section{Public Inquiries office}

USGS Public Inquiries Offices provide general information about the Geological Survey's programs and its publications, and they sell, over the counter, maps of local areas and books 
of local and general interest. The nearest Public Inquiries Office to the Oklahoma City area is:

Public Inquiries Office

U.S. Geological Survey

169 Federal Building

1961 Stout street

Denver, CO 80294

(303) $844-4169$

\section{Data Information Services:}

The Oklahoma District also manages the following data information services that provide water-resources data in an easy-to-use and readily available form:

NAWDEX (NAtional Water Data EXchange) provides information on the location and types of data available on water and related subjects. (District contact for this information is Lionel D. Mize.)

WATSTORE (WATer Data STOrage and REtrieval System) provides the following types of information:

1. Well depth, depth to water, well yield, name of aquifer, and well-construction data for nearly 20,000 ground-water wells in Oklahoma.

2. Current discharge and quality-of-water data from about 150 streams, lakes, and springs in Oklahoma.

3. Current peak-flow data from about 40 partial-record stations in Oklahoma.

(District contacts for this information are Joanne $\mathrm{K}$. Kurklin and Donna I. Runkle.)

General information on Oklahoma water resources and publications:

For general information of water resources and availability of publications dealing with Oklahoma, contact:

Charles R. Burchett, District Chief

U.S. Geological Survey, Water Resources Division

Rm. 621, 215 Dean A. McGee Avenue

Oklahoma City, OK 73102

(405) 231-4256 
Table 1.--Alphabetical listing of current and historical gaging stations maintained by the U.S. Geological Survey

September 30, 1987

[Symbols defined on last page of report]

\begin{tabular}{|c|c|c|c|c|c|c|c|}
\hline $\begin{array}{l}\text { Station } \\
\text { Number }\end{array}$ & Station & $\begin{array}{c}\text { Type } \\
\text { of } \\
\text { Data }\end{array}$ & $\begin{array}{l}\text { Q.W. } \\
\text { Para- } \\
\text { meters }\end{array}$ & $\begin{array}{l}\text { Stage } \\
\text { Source }\end{array}$ & $\begin{array}{l}\text { Coop- } \\
\text { orator }\end{array}$ & $\begin{array}{c}\text { Drainage } \\
\text { Area } \\
\text { (mile) }\end{array}$ & Period of Record \\
\hline $\begin{array}{l}\emptyset 724355 \emptyset \\
\varnothing 724355 \emptyset \\
\emptyset 724216 \emptyset \\
\emptyset 724216 \emptyset \\
\varnothing 7329849\end{array}$ & $\begin{array}{l}\text { Adams } C k n r \text { Beggs } \\
\text { Adams } C k n r \text { Beggs } \\
\text { A labama } C k n r \text { Weleotka } \\
\text { A labama Ck nr Weleotka } \\
\text { Antelope Springs at Sulphur }\end{array}$ & $\begin{array}{l}\text { CS } \\
\text { CSR } \\
\text { CS } \\
\text { CSR } \\
\text { STR }\end{array}$ & & & $\begin{array}{l}\text { HG } \\
\text { HGJ } \\
\text { b }\end{array}$ & $\begin{array}{c}5.90 \\
5.96 \\
16.5 \\
16.5\end{array}$ & $\begin{array}{l}1965-85 \\
1965-76 \\
1965- \\
1965-83 \\
1986-\end{array}$ \\
\hline $\begin{array}{l}\emptyset 723265 \emptyset \\
\varnothing 722943 \emptyset \\
\varnothing 732989 \emptyset \\
\varnothing 724234 \emptyset \\
\emptyset 716421 \emptyset\end{array}$ & $\begin{array}{l}\text { Aqua Frio Ck } n r \text { Felt } \\
\text { Arbeca } C k n r \text { Allen } \\
\text { Arbuckle } L n r \text { Dougherty } \\
\text { Arcadia } L n r \text { Arcadia } \\
\text { Ark } R \text { at Keystone } L \text {. TW }\end{array}$ & $\begin{array}{l}\text { CSR } \\
\text { CS } \\
\text { RES } \\
\text { RES } \\
\text { STGU }\end{array}$ & & $\begin{array}{l}D C P \\
D C P \\
D C P\end{array}$ & $\begin{array}{l}c \\
C \\
C\end{array}$ & $\begin{array}{l}31.0 \\
2.26\end{array}$ & $\begin{array}{l}1964-75 \\
1964-74 \\
1986- \\
1986-\end{array}$ \\
\hline $\begin{array}{l}67194651 \\
67194651 \\
6719465 \varnothing \\
6716446 \emptyset \\
6716446 \emptyset\end{array}$ & $\begin{array}{l}\text { Ark } R \text { at Wober Falls } L D \\
\text { Ark } R \text { at Wober Falls } L D \\
\text { Ark } R \text { at Wober Falls } L D \text { (US) } \\
\text { Arkansas } R \text { Sand Springs Br nr Tulsa } \\
\text { Arkansas } R \text { Sand Springs Br nr Tulsa }\end{array}$ & $\begin{array}{l}\text { QWD } \\
\text { STGU } \\
\text { STGU } \\
\text { QWH } \\
\text { QWMP }\end{array}$ & $\begin{array}{l}\mathrm{CP} \\
\mathrm{BCNP} \\
\mathrm{CNP}\end{array}$ & $D C P$ & $\begin{array}{l}c \\
c\end{array}$ & $\begin{array}{l}97049 . \\
97049 . \\
97049 . \\
74616 . \\
74615\end{array}$ & $\begin{array}{l}1949,57-63 \\
1986- \\
1986- \\
1946-77 \\
1976-77\end{array}$ \\
\hline $\begin{array}{l}\sigma 71644 \varnothing \emptyset \\
671465 \varnothing \emptyset \\
671465 \varnothing \emptyset \\
671465 \varnothing \emptyset \\
\sigma 716552 \emptyset\end{array}$ & $\begin{array}{l}\text { Arkansas } R \text { Sand Springs Br } n r \text { Tulsa } \\
\text { Arkansas } R \text { at Arkansas City KS } \\
\text { Arkansas } R \text { at Arkansas City KS } \\
\text { Arkansas } R \text { at Arkansas City KS } \\
\text { Arkansas } R \text { at Bixby }\end{array}$ & $\begin{array}{l}\text { SED } \\
\text { STRP } \\
\text { QWD } \\
\text { QWMP } \\
\text { QWD }\end{array}$ & $\begin{array}{l}\text { S } \\
\text { CNP } \\
\text { CNP } \\
\text { CP }\end{array}$ & BDT & $w$ & $\begin{array}{l}74615 \\
43713 \\
43713 \\
43713\end{array}$ & $\begin{array}{l}1974-77 \\
1955-76 \\
1952- \\
1972-75 \\
1949\end{array}$ \\
\hline $\begin{array}{l}\emptyset 725656 \emptyset \\
67148128 \\
\emptyset 716561 \emptyset \\
671525 \varnothing \emptyset \\
6715250 \emptyset\end{array}$ & $\begin{array}{l}\text { Arkansas } R \text { at Dam } 13 n r \text { Van Buren } \\
\text { Arkansas } R \text { at Kaw City } \\
\text { Arkansas } R \text { at Muskogee } \\
\text { Arkansas } R \text { at Ralston } \\
\text { Arkansas } R \text { at Ralston }\end{array}$ & $\begin{array}{l}\text { QWMP } \\
\text { QWD } \\
\text { QWP } \\
\text { STR } \\
\text { QWH }\end{array}$ & $\begin{array}{l}\text { CNP } \\
\text { CNP } \\
\text { CP } \\
\text { BCNP }\end{array}$ & $D C P$ & C & $\begin{array}{r}156547 . \\
8670 . \\
54465 \\
54465\end{array}$ & $\begin{array}{l}1976-77 \\
1949-51 \\
1957,62,63 \\
1925- \\
1950-63,65-80\end{array}$ \\
\hline $\begin{array}{l}\emptyset 71525 \varnothing \emptyset \\
671525 \varnothing \emptyset \\
\emptyset 71525 \varnothing \emptyset \\
\varnothing 71525 \varnothing \emptyset \\
\emptyset 71645 \varnothing \emptyset\end{array}$ & $\begin{array}{l}\text { Arkansas } R \text { at } R a l s t o n \\
\text { Arkansas } R \text { at } R a l s t o n \\
\text { Arkansas } R \text { at } R a l s t o n \\
\text { Arkansas } R \text { at } R a l s t o n \\
\text { Arkansas } R \text { at Tulsa }\end{array}$ & $\begin{array}{l}\text { QWMP } \\
\text { SED } \\
\text { QWP } \\
\text { QWD } \\
\text { STR }\end{array}$ & $\begin{array}{l}\text { CNP } \\
S \\
\text { BCNP } \\
P\end{array}$ & $\begin{array}{l}\text { OBS } \\
\text { DCP }\end{array}$ & $\begin{array}{l}\text { CAE } \\
\text { CAE } \\
C A \\
C\end{array}$ & $\begin{array}{l}54465 . \\
54465 \\
54465 \\
54465 \\
74615\end{array}$ & $\begin{array}{l}1973-79 \\
* * * 1973- \\
1981- \\
1950-63,68- \\
1925-\end{array}$ \\
\hline $\begin{array}{l}\emptyset 71645 \varnothing \emptyset \\
671645 \varnothing \emptyset \\
\emptyset 71645 \varnothing \emptyset \\
671646 \varnothing \emptyset \\
\emptyset 71646 \varnothing \emptyset\end{array}$ & $\begin{array}{l}\text { Arkansas } R \text { at Tulsa } \\
\text { Arkansas } R \text { at Tulsa } \\
\text { Arkansas } R \text { at Tulsa } \\
\text { Arkansas } R \text { at Tulsa } \\
\text { Arkansas } R \text { at Tulsa }\end{array}$ & $\begin{array}{l}\text { QWH } \\
\text { QWMP } \\
\text { SED } \\
\text { QWP } \\
\text { QWD }\end{array}$ & $\begin{array}{l}\text { BCNP } \\
\text { CNP } \\
\text { S } \\
\text { BCNP } \\
P\end{array}$ & OBS & $\begin{array}{l}C A \\
C A \\
C\end{array}$ & $\begin{array}{l}74615 \\
74615 \\
74615 \\
74615 \\
74615\end{array}$ & $\begin{array}{l}1960-61,77-8 \varnothing \\
1977 \\
1977- \\
1960-61,81- \\
1977-\end{array}$ \\
\hline 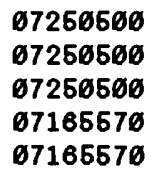 & $\begin{array}{l}\text { Arkansas } R \text { at Van Buren } A R \\
\text { Arkansas } R \text { at Van Buren } A R \\
\text { Arkansas } R \text { at Van Buren } A R \\
\text { Arkansas } R n r \text { Haskell } \\
\text { Arkansas } R n r \text { Haskell }\end{array}$ & $\begin{array}{l}\text { QWP } \\
\text { QWMP } \\
\text { STGU } \\
\text { STR } \\
\text { QWP }\end{array}$ & $\begin{array}{l}\text { CP } \\
\text { CNP } \\
\text { BCNP }\end{array}$ & $\begin{array}{l}D C P \\
D C P\end{array}$ & $\begin{array}{l}C \\
C\end{array}$ & $\begin{array}{l}160482 \\
160482 \\
160482 \\
76473 \\
76473\end{array}$ & $\begin{array}{l}1960-61 \\
1973-75 \\
1986- \\
1972- \\
1974-75\end{array}$ \\
\hline
\end{tabular}

* Continuous streamflow records for this period.

* Some records in intervening years.

*** Some records may have been collected previously. 
Table 1.--Alphabetical listing of current and historical gaging stations maintained by the U.S. Geological Survey

September 30, 1987--Continued

[Symbols defined on last page of report]

\begin{tabular}{|c|c|c|c|c|c|c|c|}
\hline $\begin{array}{l}\text { Station } \\
\text { Number }\end{array}$ & Station & $\begin{array}{l}\text { Type } \\
\text { of } \\
\text { Data }\end{array}$ & $\begin{array}{l}\text { Q.W. } \\
\text { Para- } \\
\text { meters }\end{array}$ & $\begin{array}{l}\text { Stage } \\
\text { Source }\end{array}$ & $\begin{array}{l}\text { Coop- } \\
\text { erator }\end{array}$ & $\begin{array}{c}\text { Drainage } \\
\text { Areaz } \\
\left(m i l e^{2}\right)\end{array}$ & Period of Record \\
\hline $\begin{array}{l}\varnothing 716557 \emptyset \\
\varnothing 716557 \emptyset \\
\varnothing 71945 \varnothing \emptyset \\
\varnothing 71945 \varnothing \emptyset \\
\varnothing 71945 \varnothing \emptyset\end{array}$ & $\begin{array}{l}\text { Arkansas } R n r \text { Haskell } \\
\text { Arkansas } R n r \text { Haskell } \\
\text { Arkansas } R n r \text { Muskogee } \\
\text { Arkansas } R n r \text { Muskogee } \\
\text { Arkansas } R \quad n r \text { Muskogee }\end{array}$ & $\begin{array}{l}\text { QWMP } \\
\text { SED } \\
\text { STR } \\
\text { QWP } \\
\text { QWMP }\end{array}$ & $\begin{array}{l}\text { CNP } \\
S \\
\text { CP } \\
\text { CNP }\end{array}$ & BDT & & $\begin{array}{l}75473 \\
75473 \\
96674 \\
96674 \\
96674\end{array}$ & $\begin{array}{l}1974-79 \\
1974-76 \\
1926-7 \varnothing \\
195763 \\
1973-79\end{array}$ \\
\hline $\begin{array}{l}\emptyset 71945 \varnothing \emptyset \\
\varnothing 714814 \emptyset \\
\varnothing 714814 \emptyset \\
\varnothing 714814 \varnothing \\
\varnothing 72465 \varnothing \emptyset\end{array}$ & $\begin{array}{l}\text { Arkansas } R n r \text { Muskogee } \\
\text { Arkansas } R n r \text { Ponca City } \\
\text { Arkansas } R n r \text { Ponca City } \\
\text { Arkansas } R n r \text { Ponca City } \\
\text { Arkansas } R n r \text { Sallisaw }\end{array}$ & $\begin{array}{l}\text { STGU } \\
\text { STR } \\
\text { QWMP } \\
\text { SED } \\
\text { STRP }\end{array}$ & $\begin{array}{l}\text { CNP } \\
\mathbf{S}\end{array}$ & $D C P$ & $\begin{array}{l}\mathrm{C} \\
\mathrm{C} \\
\mathrm{C}\end{array}$ & $\begin{array}{l}96674 . \\
46530 \\
46530 \\
46530 \\
147767\end{array}$ & $\begin{array}{l}1986- \\
1977- \\
1978-79 \\
1978- \\
1948-7 \varnothing\end{array}$ \\
\hline $\begin{array}{l}\varnothing 72465 \varnothing \emptyset \\
\varnothing 71656 \varnothing \emptyset \\
\varnothing 73246 \varnothing \emptyset \\
\varnothing 73246 \varnothing \emptyset \\
\varnothing 71969 \varnothing \emptyset\end{array}$ & $\begin{array}{l}\text { Arkansas } R n r \text { Sallisaw } \\
\text { Arkansas } R n r \text { Tullahasseo } \\
\text { Barnitz Ck } n r \text { Arapaho } \\
\text { Barnitz Ck nr Arapaho } \\
\text { Baron Fk at Dutch Mills Ark }\end{array}$ & $\begin{array}{l}\text { QWD } \\
\text { STR } \\
\text { STR } \\
\text { QWP } \\
\text { QWP }\end{array}$ & $\begin{array}{l}\mathrm{CP} \\
\mathrm{CP} \\
\mathrm{C}\end{array}$ & & & $\begin{array}{r}147767 \\
75815 \\
243 \\
243 \\
43 .\end{array}$ & $\begin{array}{l}195759-63 \\
1971-72 \\
1943-64 \\
1952,55 \\
1959-61\end{array}$ \\
\hline $\begin{array}{l}\varnothing 7197 \varnothing \varnothing \emptyset \\
67197 \varnothing \varnothing \emptyset \\
\varnothing 7197 \varnothing \varnothing \emptyset \\
\varnothing 7194612 \\
\varnothing 73 \varnothing \varnothing 15 \emptyset\end{array}$ & $\begin{array}{l}\text { Baron Fk at EIdon } \\
\text { Baron Fk at EIdon } \\
\text { Baron Fk at EIdon } \\
\text { Bayou Manard nr Ft Gibson } \\
\text { Bear Ck nr Vinson }\end{array}$ & $\begin{array}{l}\text { STR } \\
\text { QWP } \\
\text { QWMP } \\
\text { QWP } \\
\text { CS }\end{array}$ & $\begin{array}{l}\text { CP } \\
C N P \\
C P\end{array}$ & DCP & CJG & $\begin{array}{l}307 . \\
367 . \\
367 . \\
7.24\end{array}$ & $\begin{array}{l}1948- \\
1958-60 \\
1975-79 \\
1961 \\
1964-85\end{array}$ \\
\hline $\begin{array}{l}\emptyset 736 \varnothing 15 \emptyset \\
\varnothing 72324 \varnothing \emptyset \\
\varnothing 716238 \emptyset \\
\varnothing 7148126 \\
\varnothing 731276 \varnothing\end{array}$ & $\begin{array}{l}\text { Bear Ck nr Vinson } \\
\text { Beaver (Sand) Ck nr Texhoma } \\
\text { Beaver Ck (E Br) nr Grainola } \\
\text { Beaver Ck nr Kaw City } \\
\text { Beaver Ck nr Lawton }\end{array}$ & $\begin{array}{l}\text { CSR } \\
\text { LF } \\
\text { QWP } \\
\text { QWP } \\
\text { QWP }\end{array}$ & $\begin{array}{l}C P \\
C O P \\
C P\end{array}$ & & & $\begin{array}{l}7.24 \\
217 .\end{array}$ & $\begin{array}{l}1964-76 \\
1967-71 \\
195 \varnothing \\
1950,64-56 \\
1948,61\end{array}$ \\
\hline $\begin{array}{l}\varnothing 73137 \varnothing 2 \\
\varnothing 73135 \varnothing \emptyset \\
\varnothing 73136 \varnothing \emptyset \\
\varnothing 73135 \varnothing \emptyset \\
\varnothing 73135 \varnothing \varnothing\end{array}$ & $\begin{array}{l}\text { Beaver Ck nr Ryan } \\
\text { Beaver Ck nr Waurika } \\
\text { Beaver Ck nr Waurika } \\
\text { Beaver Ck nr Waurika } \\
\text { Beaver Ck nr Waurika }\end{array}$ & $\begin{array}{l}\text { QWP } \\
\text { STR } \\
\text { QWP } \\
\text { QWMP } \\
\text { SED }\end{array}$ & $\begin{array}{l}C P \\
C P \\
C N P \\
S\end{array}$ & & C & $\begin{array}{l}563 . \\
563 . \\
663 . \\
563 .\end{array}$ & $\begin{array}{l}1960-61 \\
1953- \\
1953-66 \\
1975-79 \\
1953-\end{array}$ \\
\hline $\begin{array}{l}\varnothing 7234 \varnothing \varnothing \emptyset \\
\varnothing 7234 \varnothing \varnothing \emptyset \\
\varnothing 7234 \varnothing \varnothing \emptyset \\
\varnothing 7234 \varnothing \varnothing \emptyset \\
\varnothing 7234 \varnothing \varnothing \emptyset\end{array}$ & $\begin{array}{l}\text { Beaver } R \text { at Beaver } \\
\text { Beaver } R \text { at Beaver } \\
\text { Beaver } R \text { at Beaver } \\
\text { Beaver } R \text { at Beaver } \\
\text { Beaver } R \text { at Beaver }\end{array}$ & $\begin{array}{l}\text { STR } \\
\text { QWD } \\
\text { QWMP } \\
\text { SED } \\
\text { QWP }\end{array}$ & $\begin{array}{l}\text { BCNP } \\
\text { CNP } \\
S \\
\text { BCNP }\end{array}$ & $D C P$ & $\begin{array}{l}A \\
A\end{array}$ & $\begin{array}{l}7955 . \\
7955 . \\
7955 . \\
7955 . \\
7955 .\end{array}$ & $\begin{array}{l}1937- \\
1952,58-59,62-82 \\
1973-77 \\
1974- \\
1983-\end{array}$ \\
\hline $\begin{array}{l}\emptyset 7232246 \\
\varnothing 723225 \varnothing \\
\varnothing 723225 \varnothing \\
\varnothing 72346 \varnothing \emptyset \\
\varnothing 72325 \varnothing \varnothing\end{array}$ & $\begin{array}{l}\text { Beaver } R \text { at Felt } \\
\text { Beaver } R n r \text { Felt } \\
\text { Beaver } R n r \text { Folt } \\
\text { Beaver } R n r \text { Fort Supply } \\
\text { Beaver } R n r \text { Guymon }\end{array}$ & $\begin{array}{l}\text { QWMP } \\
\text { CS } \\
\text { STR } \\
\text { STGU } \\
\text { STR }\end{array}$ & CNP & DCP & $\begin{array}{l}\text { JG } \\
\mathrm{C}\end{array}$ & $\begin{array}{l}850 . \\
879 . \\
879 . \\
9616 . \\
2139 .\end{array}$ & $\begin{array}{l}1977 \\
1972-79 \\
1979- \\
* 1937-51,61-79 \\
1937-\end{array}$ \\
\hline
\end{tabular}

* Continuous streamflow records for this period.

** Some records in intervening years.

*** Some records may have been collected previously. 
Table 1.--Alphabetical listing of current and historical gaging stations maintained by the U.S. Geological Survey

September $30,1987-$-Continued

[Symbols defined on last page of report]

\begin{tabular}{|c|c|c|c|c|c|c|c|}
\hline $\begin{array}{l}\text { Station } \\
\text { Number }\end{array}$ & Station & $\begin{array}{l}\text { Type } \\
\text { of } \\
\text { Data }\end{array}$ & $\begin{array}{l}\text { Q.W. } \\
\text { Para- } \\
\text { meters }\end{array}$ & $\begin{array}{l}\text { Stage } \\
\text { Source }\end{array}$ & $\begin{array}{l}\text { Coop- } \\
\text { erator }\end{array}$ & $\begin{array}{c}\text { Drainage } \\
\text { Area } \\
\left(m i l \theta^{2}\right)\end{array}$ & Period of Record \\
\hline $\begin{array}{l}\varnothing 72325 \varnothing \emptyset \\
\varnothing 72325 \varnothing \emptyset \\
\varnothing 72325 \varnothing \emptyset \\
\varnothing 723321 \emptyset \\
\varnothing 723321 \emptyset\end{array}$ & $\begin{array}{l}\text { Beaver } R \mathrm{nr} \text { Guymon } \\
\text { Beaver } R \mathrm{nr} \text { Guymon } \\
\text { Beaver } R \mathrm{nr} \text { Guymon } \\
\text { Beaver } R \mathrm{nr} \text { Hardesty } \\
\text { Beaver } R \mathrm{nr} \text { Hardesty }\end{array}$ & $\begin{array}{l}\text { QWD } \\
\text { QWMP } \\
\text { SED } \\
\text { STR } \\
\text { SED }\end{array}$ & $\begin{array}{l}\text { BCNP } \\
\text { CNP } \\
S \\
S\end{array}$ & & $\begin{array}{l}c \\
c \\
c\end{array}$ & $\begin{array}{l}2139 . \\
2139 . \\
2139 . \\
477 \varnothing . \\
4770 .\end{array}$ & $\begin{array}{l}1952,54-63,68-79 \\
1973-77 \\
1975- \\
1978- \\
1978-\end{array}$ \\
\hline $\begin{array}{l}\emptyset 723263 \emptyset \\
\varnothing 723263 \emptyset \\
\varnothing 723261 \emptyset \\
\varnothing 72425 \varnothing \emptyset \\
\varnothing 72425 \varnothing \emptyset\end{array}$ & $\begin{array}{l}\text { Beaver } R \mathrm{nr} \text { Hooker } \\
\text { Beaver } R \mathrm{nr} \text { Hooker } \\
\text { Beaver } R \mathrm{nr} \text { Optima } \\
\text { Bell cow Ck at Chandler } \\
\text { Bell cow Ck at Chandler }\end{array}$ & $\begin{array}{l}\text { QWP } \\
\text { QWMP } \\
\text { QWP } \\
\text { STR } \\
\text { QWP }\end{array}$ & $\begin{array}{l}\text { CP } \\
\text { CNP } \\
C P \\
C P\end{array}$ & & & $\begin{array}{l}3017 . \\
3017 \\
46 . \varnothing \\
46 . \varnothing\end{array}$ & $\begin{array}{l}1972-73,75-79 \\
1977 \\
1972-73 \\
1948-56 \\
1949-54\end{array}$ \\
\hline $\begin{array}{l}\varnothing 72378 \varnothing \emptyset \\
\varnothing 72378 \varnothing \emptyset \\
\varnothing 72378 \varnothing \emptyset \\
\varnothing 724663 \emptyset \\
\varnothing 733722 \varnothing\end{array}$ & $\begin{array}{l}\text { Bent Ck nr Seiling } \\
\text { Bent Ck nr Seiling } \\
\text { Bent Ck nr Seiling } \\
\text { Big Black Fox Ck nr Long } \\
\text { Big Br nr Ringold }\end{array}$ & $\begin{array}{l}\text { CS } \\
\text { QWP } \\
\text { STR } \\
\text { CS } \\
\text { CS }\end{array}$ & $\mathrm{CP}$ & & $\begin{array}{l}J G \\
G J \\
H G\end{array}$ & $\begin{array}{l}139 . \\
139 . \\
139 . \\
5.32 \\
1.99\end{array}$ & $\begin{array}{l}* 1966-70,71- \\
1956 \\
1966-7 \varnothing \\
1964-85 \\
1964-74\end{array}$ \\
\hline $\begin{array}{l}\varnothing 7196597 \\
67196597 \\
\varnothing 7196597 \\
\varnothing 7191 \varnothing \varnothing \emptyset \\
\varnothing 7191 \varnothing \varnothing \emptyset\end{array}$ & $\begin{array}{l}\text { Big Cabin Ck Trib nr Welch } \\
\text { Big Cabin Ck Trib nr Welch } \\
\text { Big Cabin Ck Trib nr Welch } \\
\text { Big Cabin Ck nr Big Cabin } \\
\text { Big Cabin Ck nr Big Cabin }\end{array}$ & $\begin{array}{l}\text { QWMP } \\
\text { PR } \\
\text { SED } \\
\text { STR } \\
\text { QWP }\end{array}$ & $\begin{array}{l}\mathrm{CP} \\
\mathrm{S} \\
\mathrm{CP}\end{array}$ & DCP & LGC & $\begin{array}{l}466 . \\
466 .\end{array}$ & $\begin{array}{l}1980-83 \\
1980-83 \\
1980-83 \\
1947- \\
1951-60,64-71\end{array}$ \\
\hline $\begin{array}{l}\varnothing 7191606 \\
\varnothing 71966 \varnothing \emptyset \\
\varnothing 7196650 \\
67196596 \\
\varnothing 7196595\end{array}$ & $\begin{array}{l}\text { Big Cabin Ck nr Big Cabin } \\
\text { Big Cabin Ck nr Pyramid Corners } \\
\text { Big Cabin Ck nr Vinita } \\
\text { Big Cabin Ck nr Welch } \\
\text { Big Cabin Ck nr Welch }\end{array}$ & $\begin{array}{l}\text { QWMP } \\
\text { CS } \\
\text { QWP } \\
\text { QWMP } \\
\text { PR }\end{array}$ & $\begin{array}{l}C N P \\
C P \\
C P\end{array}$ & & & $\begin{array}{l}466 . \\
71.1 \\
28.1 \\
28.1\end{array}$ & $\begin{array}{l}1976-77 \\
1963-72 * *, 73-79 \\
1949-51 \\
198 \emptyset-83 \\
1980-83\end{array}$ \\
\hline $\begin{array}{l}\emptyset 7196596 \\
\emptyset 717122 \emptyset \\
\varnothing 7328 \emptyset 3 \emptyset \\
\varnothing 717865 \emptyset \\
\varnothing 7176465\end{array}$ & $\begin{array}{l}\text { Big Cabin Ck nr Welch } \\
\text { Big Ck nr Nowata } \\
\text { Big Dry Ck nr Alex } \\
\text { Billy Ck Trib nr Wagoner } \\
\text { Birch Ck blw Birch Lake nr Barnsdall }\end{array}$ & $\begin{array}{l}\text { SED } \\
\text { QWP } \\
\text { CS } \\
\text { CS } \\
\text { STR }\end{array}$ & $\begin{array}{l}S \\
C P\end{array}$ & & $C$ & $\begin{array}{r}28.1 \\
7.57 \\
5.71 \\
66.0\end{array}$ & $\begin{array}{l}1980-83 \\
1952-53,59 \\
1961-74 \\
1966-72 \\
1978-\end{array}$ \\
\hline $\begin{array}{l}\varnothing 7176466 \\
\varnothing 717646 \varnothing \\
\varnothing 71776 \varnothing \emptyset \\
\varnothing 71776 \varnothing \emptyset \\
\varnothing 71765 \varnothing \emptyset\end{array}$ & $\begin{array}{l}\text { Birch Ck nr Barnsdall } \\
\text { Birch Lake nr Barnsdall } \\
\text { Bird Ck at 66th St at Tulsa } \\
\text { Bird Ck at 66th St at Tulsa } \\
\text { Bird Ck at Avant }\end{array}$ & $\begin{array}{l}\text { QWP } \\
\text { RES } \\
\text { STR } \\
\text { QWH } \\
\text { STR }\end{array}$ & $\mathrm{CP}$ & $\begin{array}{l}D C P \\
D C P\end{array}$ & $\begin{array}{l}C \\
P \\
P \\
C\end{array}$ & $\begin{array}{l}66 . \emptyset \\
364 .\end{array}$ & $\begin{array}{l}1965-66 \\
1977- \\
1986- \\
1986- \\
1945-\end{array}$ \\
\hline $\begin{array}{l}\emptyset 71765 \varnothing \emptyset \\
\varnothing 71765 \varnothing \emptyset \\
\varnothing 71784 \varnothing \emptyset \\
\varnothing 717635 \emptyset \\
\varnothing 7178 \varnothing 5 \emptyset\end{array}$ & $\begin{array}{l}\text { Bird Ck at Avant } \\
\text { Bird Ck at Avant } \\
\text { Bird Ck at Catoosa } \\
\text { Bird Ck nr Barnsdall } \\
\text { Bird Ck nr Catoosa }\end{array}$ & $\begin{array}{l}\text { QWP } \\
\text { QWMP } \\
\text { QWMP } \\
\text { QWP } \\
\text { QWP }\end{array}$ & $\begin{array}{l}\text { CP } \\
\text { CNP } \\
\text { CNP } \\
\text { CP } \\
C P\end{array}$ & & JG & $\begin{array}{l}364 . \\
364 .\end{array}$ & $\begin{array}{l}1965-66 \\
1973-79 \\
1978-79 \\
1949-53 \\
1965-\end{array}$ \\
\hline
\end{tabular}

* Continuous streamflow records for this period.

** Some records in intervening years.

*** Some records may have been collected previously. 
Table 1.--Alphabetical listing of current and historical gaging stations maintained by the U.S. Geological Survey

September s0, 1987--Continued

[Symbols defined on last page of report]

\begin{tabular}{|c|c|c|c|c|c|c|c|}
\hline $\begin{array}{r}\text { Station } \\
\text { Number }\end{array}$ & Station & $\begin{array}{l}\text { Type } \\
\text { of } \\
\text { Data }\end{array}$ & $\begin{array}{l}\text { Q.W. } \\
\text { Para- } \\
\text { meters }\end{array}$ & $\begin{array}{l}\text { Stage } \\
\text { Source }\end{array}$ & $\begin{array}{l}\text { Coop- } \\
\text { erator }\end{array}$ & $\begin{array}{c}\text { Drainage } \\
\text { Area } \\
\left(m i l e^{2}\right)\end{array}$ & Period of Record \\
\hline 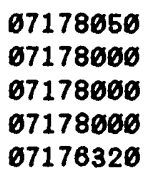 & $\begin{array}{l}\text { Bird Ck nr Catoosa } \\
\text { Bird Ck nr Owasso } \\
\text { Bird Ck nr Owasso } \\
\text { Bird Ck nr Owasso } \\
\text { Bird Ck nr Pawhuska }\end{array}$ & $\begin{array}{l}\text { QWMP } \\
\text { STR } \\
\text { QWP } \\
\text { QWH } \\
\text { QWP }\end{array}$ & $\begin{array}{l}C N P \\
C P \\
P \\
C P\end{array}$ & & $\mathbf{P}$ & $\begin{array}{r}1680 . \\
1622 . \\
1622 . \\
1622 . \\
167 .\end{array}$ & $\begin{array}{l}1975-79 \\
1936-39,86- \\
1948-53 \\
1986- \\
1952-53\end{array}$ \\
\hline $\begin{array}{l}\emptyset 7176910 \\
6717750 \emptyset \\
67177560 \\
67177560 \\
67177560\end{array}$ & $\begin{array}{l}\text { Bird Ck nr Skiatook } \\
\text { Bird Ck nr Sperry } \\
\text { Bird Ck nr Sperry } \\
\text { Bird Ck nr Sperry } \\
\text { Bird Ck nr Sperry }\end{array}$ & $\begin{array}{l}\text { QWP } \\
\text { STR } \\
\text { QWD } \\
\text { QWMP } \\
\text { QWH }\end{array}$ & $\begin{array}{l}\mathrm{CP} \\
\mathrm{CP} \\
\mathrm{CNP} \\
\mathrm{P}\end{array}$ & $D C P$ & $P$ & $\begin{array}{l}965 . \\
905 . \\
965 . \\
965 .\end{array}$ & $\begin{array}{l}1948-53 \\
1938- \\
1952-53, * *, 64-76 \\
1976-77 \\
1986-\end{array}$ \\
\hline $\begin{array}{l}67229655 \\
67229655 \\
672997 \varnothing 2 \\
67299765 \\
6715252 \emptyset\end{array}$ & $\begin{array}{l}\text { Bishop } \mathrm{Cr} \text { at Norman } \\
\text { Bishop } \mathrm{Cr} \text { at Norman } \\
\text { Bitter Ck nr Hollis } \\
\text { Bitter Ck nr Hollis } \\
\text { Black Bear Ck Trib nr Garber }\end{array}$ & $\begin{array}{l}\text { CSR } \\
\text { CSR } \\
\text { STR } \\
\text { CS } \\
\text { CS }\end{array}$ & & & J & $\begin{array}{r}11.3 \\
0.97\end{array}$ & $\begin{array}{l}1987- \\
1988- \\
1987- \\
1964-72 \\
1964-75\end{array}$ \\
\hline $\begin{array}{l}67152520 \\
671536 \varnothing \emptyset \\
671536 \varnothing \emptyset \\
671536 \varnothing \emptyset \\
671536 \varnothing 0\end{array}$ & $\begin{array}{l}\text { Black Bear Ck Trib nr Garber } \\
\text { Black Bear Ck at Pawnee } \\
\text { Black Bear Ck at Pawnee } \\
\text { Black Bear Ck at Pawnee } \\
\text { Black Bear Ck at Pawnee }\end{array}$ & $\begin{array}{l}\text { QWP } \\
\text { STR } \\
\text { QWP } \\
\text { QWMP } \\
\text { SED }\end{array}$ & $\begin{array}{l}C P \\
C P \\
C N P \\
S\end{array}$ & $D C P$ & C & $\begin{array}{l}\quad .97 \\
576 . \\
576 . \\
576 . \\
576\end{array}$ & $\begin{array}{l}1958-59 \\
1944- \\
1952-53,56-59,61-71 \\
1978-79 \\
1978-\end{array}$ \\
\hline $\begin{array}{l}67152950 \\
67152590 \\
672367 \varnothing 8 \\
67311200 \\
67311200\end{array}$ & $\begin{array}{l}\text { Black Bear Ck nr Morrison } \\
\text { Black Bear Ck nr Perry } \\
\text { Blacksmith Ck nr Pearson } \\
\text { Blue Beaver Ck nr Cache } \\
\text { Blue Beaver Ck nr Cache }\end{array}$ & $\begin{array}{l}\text { QWP } \\
\text { QWP } \\
\text { QWP } \\
\text { STR } \\
\text { QWP }\end{array}$ & $\begin{array}{l}\mathrm{CP} \\
\mathrm{CP} \\
\mathrm{CP} \\
\mathrm{BCNP}\end{array}$ & & $\begin{array}{l}A \\
A\end{array}$ & $\begin{array}{l}24.6 \\
24.6\end{array}$ & $\begin{array}{l}1951,58-59 \\
1958,58-59 \\
1961 \\
1964- \\
1965-\end{array}$ \\
\hline 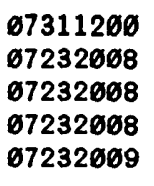 & $\begin{array}{l}\text { Blue Beaver Ck nr Cache } \\
\text { Blue Ck Trib A nr Blocker } \\
\text { Blue Ck Trib A nr Blocker } \\
\text { Blue Ck Trib A nr Blocker } \\
\text { Blue Ck Trib B nr Blocker }\end{array}$ & $\begin{array}{l}\text { SED } \\
\text { PR } \\
\text { QWP } \\
\text { SED } \\
\text { PR }\end{array}$ & $\begin{array}{l}S \\
\text { CNP } \\
S\end{array}$ & & A & $\begin{array}{r}24.6 \\
4.67 \\
4.67 \\
4.67 \\
0.22\end{array}$ & $\begin{array}{l}1965- \\
1978-81 \\
1978-81 \\
1978-81 \\
1976-78\end{array}$ \\
\hline 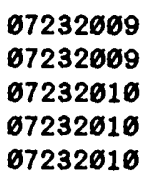 & $\begin{array}{l}\text { Blue Ck Trib B nr Blocker } \\
\text { Blue Ck Trib B nr Blocker } \\
\text { Blve Ck nr Blocker } \\
\text { Blue Ck nr Blocker } \\
\text { Blue Ck nr Blocker }\end{array}$ & $\begin{array}{l}\text { QWP } \\
\text { SED } \\
\text { STR } \\
\text { QWP } \\
\text { SED }\end{array}$ & $\begin{array}{l}\text { CNP } \\
S \\
\text { CNP } \\
S\end{array}$ & & & $\begin{array}{l}0.22 \\
0.22 \\
12.1 \\
12.1 \\
12.1\end{array}$ & $\begin{array}{l}1976-78 \\
1976-78 \\
1976-82 \\
1976-82 \\
1976-81\end{array}$ \\
\hline $\begin{array}{l}6733245 \emptyset \\
6733235 \emptyset \\
6733246 \emptyset \\
673324 \varnothing \emptyset \\
6733250 \emptyset\end{array}$ & $\begin{array}{l}\text { Blue } R \text { at Armstrong } \\
\text { Blue } R \text { at Connerville } \\
\text { Blue } R \text { at Milburn } \\
\text { Blue } R \text { at Milburn } \\
\text { Blue } R \text { nr Blue }\end{array}$ & $\begin{array}{l}\text { QWMP } \\
\text { QWP } \\
\text { STR } \\
\text { QWP } \\
\text { STRP }\end{array}$ & $\begin{array}{l}\text { CNP } \\
C P \\
C P\end{array}$ & & $\begin{array}{l}J G \\
C\end{array}$ & $\begin{array}{l}224 . \\
263 . \\
203 \\
476\end{array}$ & $\begin{array}{l}1977 \\
1951-57,62 \\
1965-87 \\
1956-66 \\
1936-\end{array}$ \\
\hline
\end{tabular}

* Continuous streamflow records for this period.

* Some records in intervening years.

*** Some records may have been collected previously. 
Table 1.--Alphabetical listing of current and historical gaging stations maintained by the U.S. Geological Survey

September 30, 1987--Continued

[Symbols defined on last page of report]

\begin{tabular}{|c|c|c|c|c|c|c|c|}
\hline $\begin{array}{r}\text { Station } \\
\text { Number }\end{array}$ & Station & $\begin{array}{l}\text { Type } \\
\text { of } \\
\text { Data }\end{array}$ & $\begin{array}{l}\text { Q.W. } \\
\text { Para- } \\
\text { meters }\end{array}$ & $\begin{array}{l}\text { Stage } \\
\text { Source }\end{array}$ & $\begin{array}{l}\text { Coop- } \\
\text { erator }\end{array}$ & $\begin{array}{c}\text { Drainage } \\
\text { Area } \\
(\mathrm{mile})\end{array}$ & Period of Record \\
\hline $\begin{array}{l}\varnothing 73325 \varnothing \emptyset \\
673325 \varnothing \emptyset \\
6733239 \emptyset \\
\varnothing 715962 \emptyset \\
\varnothing 71595 \varnothing \emptyset\end{array}$ & $\begin{array}{l}\text { Blue } R \text { nr Blue } \\
\text { Blue } R \text { nr Blue } \\
\text { Blue } R n r \text { Connerville } \\
\text { Bluff Ck abv Bethany Swg Trmt PInt *OKC } \\
\text { Bluff Ck abv Lake Hefner nr OKC }\end{array}$ & $\begin{array}{l}\text { QWD } \\
\text { QWMP } \\
\text { STR } \\
\text { QWP } \\
\text { STR }\end{array}$ & $\begin{array}{l}\text { CP } \\
\text { CNP } \\
\text { CNOP }\end{array}$ & & & $\begin{array}{l}476 . \\
476 . \\
162 . \\
\quad 1.62\end{array}$ & $\begin{array}{l}1951-58,60-63 \\
1973-79 \\
1977-79 \\
1983-84 \\
195 \emptyset-58\end{array}$ \\
\hline $\begin{array}{l}\emptyset 715946 \emptyset \\
\varnothing 715964 \emptyset \\
\varnothing 715794 \emptyset \\
\varnothing 716794 \emptyset \\
\varnothing 716 \varnothing 31 \emptyset\end{array}$ & $\begin{array}{l}\text { Bluff } \mathrm{Ck} \text { at OKC } \\
\text { Bluff } \mathrm{Ck} \text { blw Bethany Swg Trmt PInt *OKC } \\
\text { Bluff Ck nr Buttermilk KS } \\
\text { Bluff Ck nr Buttermilk KS } \\
\text { Boggy Ck Trib at Garriott Rd, Enid }\end{array}$ & $\begin{array}{l}\text { CS } \\
\text { QWP } \\
\text { STR } \\
\text { QWH } \\
\text { QWP }\end{array}$ & $\begin{array}{l}\text { CNOP } \\
\text { CP } \\
\text { BCNP }\end{array}$ & & & $\begin{array}{l}1.64 \\
657 \\
657\end{array}$ & $\begin{array}{l}1974-78 \\
1983-84 \\
1973-79 \\
1973-79 \\
1975\end{array}$ \\
\hline $\begin{array}{l}\emptyset 716 \varnothing 315 \\
\varnothing 716 \varnothing 3 \emptyset \emptyset \\
6716 \varnothing 3 \emptyset \emptyset \\
\varnothing 716 \varnothing 28 \emptyset \\
\varnothing 716 \varnothing 28 \emptyset\end{array}$ & $\begin{array}{l}\text { Boggy Ck abv Swg Trtmt PInt Enid } \\
\text { Boggy } C k \text { at } 36 \text { th St, Enid } \\
\text { Boggy } C k \text { at } 30 \text { th St, Enid } \\
\text { Boggy Ck at Enid Ave, Enid } \\
\text { Boggy Ck at Enid Ave, Enid }\end{array}$ & $\begin{array}{l}\text { QWP } \\
\text { STR } \\
\text { QWD } \\
\text { STR } \\
\text { QWP }\end{array}$ & $\begin{array}{l}\text { BCNP } \\
\text { BCNP } \\
\text { BCNP }\end{array}$ & & & $\begin{array}{l}35.8 \\
35.8 \\
20.8 \\
20.8\end{array}$ & $\begin{array}{l}1975 \\
1975 \\
1975 \\
1975 \\
1975\end{array}$ \\
\hline $\begin{array}{l}\emptyset 716 \varnothing 32 \emptyset \\
\varnothing 715225 \emptyset \\
\varnothing 715225 \emptyset \\
\varnothing 7336785 \\
\varnothing 733532 \varnothing\end{array}$ & $\begin{array}{l}\text { Boggy Ck blw Swg Trtmt Plnt Enid } \\
\text { Bois D'Arc Ck nr Ponca City } \\
\text { Bois D'Arc Ck nr Ponca City } \\
\text { Bokchito Ck nr Garvin } \\
\text { Bokchito Ck nr Soper }\end{array}$ & $\begin{array}{l}\text { QWP } \\
\text { QWP } \\
\text { LF } \\
\text { CSR } \\
\text { CS }\end{array}$ & $\begin{array}{l}\text { BCNP } \\
C P\end{array}$ & & & $\begin{array}{c}100 . \\
100 . \\
2.96 \\
16.6\end{array}$ & $\begin{array}{l}1975 \\
1963,58-63 \\
1985-73 \\
1965-75 \\
1965-75\end{array}$ \\
\hline $\begin{array}{l}67249 \emptyset 73 \\
\varnothing 7249673 \\
\varnothing 7249 \emptyset 73 \\
\emptyset 72492 \emptyset \emptyset \\
\varnothing 724966 \emptyset\end{array}$ & $\begin{array}{l}\text { Brazil Ck nr Lodi } \\
\text { Brazil Ck nr Lodi } \\
\text { Brazil Ck nr Lodi } \\
\text { Brazil Ck nr Panama } \\
\text { Brazil Ck nr Red Oak }\end{array}$ & $\begin{array}{l}\text { PR } \\
\text { QWP } \\
\text { SED } \\
\text { QWP } \\
\text { PR }\end{array}$ & $\begin{array}{l}\text { CNP } \\
S \\
C P\end{array}$ & & & $\begin{array}{l}28.8 \\
28.8 \\
28.8 \\
2.74\end{array}$ & $\begin{array}{l}198 \emptyset-81 \\
198 \varnothing-81 \\
198 \varnothing-81 \\
196 \varnothing-61 \\
1978-81\end{array}$ \\
\hline $\begin{array}{l}\emptyset 7249 \emptyset 6 \emptyset \\
\varnothing 724966 \emptyset \\
\varnothing 724968 \emptyset \\
\emptyset 724968 \emptyset \\
\varnothing 724908 \emptyset\end{array}$ & $\begin{array}{l}\text { Brazil Ck nr Red Oak } \\
\text { Brazil Ck nr Red Oak } \\
\text { Brazil Ck nr Walls } \\
\text { Brazil Ck nr Walls } \\
\text { Brazil Ck nr Walls }\end{array}$ & $\begin{array}{l}\text { QWP } \\
\text { SED } \\
\text { STR } \\
\text { QWP } \\
\text { SED }\end{array}$ & $\begin{array}{l}\text { CNP } \\
S \\
\text { CNP } \\
S\end{array}$ & OBS & $A$ & $\begin{array}{r}2.74 \\
2.74 \\
69.1 \\
69.1 \\
69.1\end{array}$ & $\begin{array}{l}1978-81 \\
1978-81 \\
1978-81,84- \\
1978-81 \\
1978-81,84-\end{array}$ \\
\hline $\begin{array}{l}\emptyset 731614 \emptyset \\
\varnothing 731614 \emptyset \\
\varnothing 731614 \emptyset \\
\varnothing 724122 \emptyset \\
\varnothing 73389 \emptyset \emptyset\end{array}$ & $\begin{array}{l}\text { Brier Ck nr Powell } \\
\text { Brier Ck nr Powell } \\
\text { Brier Ck nr Powell } \\
\text { Brock Ck at Sw59, OKC } \\
\text { Broken Bow Lake nr Broken Bow }\end{array}$ & $\begin{array}{l}\text { CSR } \\
\text { CS } \\
\text { CS } \\
\text { CSR } \\
\text { RESP }\end{array}$ & & RES & $\begin{array}{l}\text { HG } \\
\text { HG } \\
\text { HG } \\
\text { C }\end{array}$ & $\begin{array}{l}12.0 \\
12.6 \\
12.0 \\
2.26 \\
754\end{array}$ & $\begin{array}{l}1965-76 \\
1965- \\
1965-86 \\
1976-81 \\
1968-\end{array}$ \\
\hline $\begin{array}{l}\emptyset 724479 \emptyset \\
\emptyset 7231975 \\
\emptyset 7231975 \\
\emptyset 7231975 \\
\emptyset 719126 \varnothing\end{array}$ & $\begin{array}{l}\text { Brooken Ck nr Enterprise } \\
\text { Brushy Ck nr Haileyville } \\
\text { Brushy Ck nr Haileyville } \\
\text { Brushy Ck nr Haileyville } \\
\text { Brushy Ck nr Jay }\end{array}$ & $\begin{array}{l}\text { CS } \\
\text { STR } \\
\text { QWP } \\
\text { SED } \\
\text { CS }\end{array}$ & $\begin{array}{l}\text { BCNP } \\
\mathrm{S}\end{array}$ & & & $\begin{array}{l}5.66 \\
139 . \\
139 . \\
139 . \\
16.0\end{array}$ & $\begin{array}{l}1964-71 \\
1978-82 \\
1978-82 \\
1978-81 \\
1965-72\end{array}$ \\
\hline
\end{tabular}

* Continuous streamflow records for this period.

* Some records in intervening years.

*** Some records may have been collected previously. 
Table 1.--Alphabetical listing of current and historical gaging stations maintained by the U.S. Geological Survey

September so, 1987--Continued

[Symbols defined on last page of report]

\begin{tabular}{|c|c|c|c|c|c|c|c|}
\hline $\begin{array}{c}\text { Station } \\
\text { Number }\end{array}$ & Station & $\begin{array}{c}\text { Type } \\
\text { of } \\
\text { Data }\end{array}$ & $\begin{array}{l}\text { Q.W. } \\
\text { Para- } \\
\text { meters }\end{array}$ & $\begin{array}{l}\text { Stage } \\
\text { Source }\end{array}$ & $\begin{array}{l}\text { Coop- } \\
\text { erator }\end{array}$ & $\begin{array}{c}\text { Drainage } \\
\text { Area } \\
\left(m i l e^{2}\right)\end{array}$ & Period of Record \\
\hline $\begin{array}{l}\emptyset 73359 \emptyset \emptyset \\
\varnothing 715796 \emptyset \\
\varnothing 715796 \varnothing \\
\varnothing 715796 \varnothing \\
\varnothing 715796 \varnothing\end{array}$ & $\begin{array}{l}\text { Buck Ck nr Moyers } \\
\text { Buffalo Ck nr Lovedale } \\
\text { Buffalo Ck nr Lovedale } \\
\text { Buffalo Ck nr Lovedale } \\
\text { Buffalo Ck nr Lovedale }\end{array}$ & $\begin{array}{l}\text { QWP } \\
\text { STR } \\
\text { QWH } \\
\text { QWMP } \\
\text { SED }\end{array}$ & $\begin{array}{l}\mathrm{CP} \\
\mathrm{CP} \\
\mathrm{CNP} \\
\mathrm{S}\end{array}$ & & JG & $\begin{array}{l}160 . \\
468 \\
468 \\
468 \\
468\end{array}$ & $\begin{array}{l}1966-67,66 \\
1966- \\
1974-79 \\
1976-79 \\
1978-79\end{array}$ \\
\hline $\begin{array}{l}\emptyset 7329847 \\
6717864 \emptyset \\
\varnothing 73314 \emptyset 1 \\
\varnothing 733141 \emptyset \\
\varnothing 73342 \varnothing \emptyset\end{array}$ & $\begin{array}{l}\text { Buffalo Spring at Sulphur } \\
\text { Bull Ck nr Inola } \\
\text { Butcher Pen Ck nr Tishomingo } \\
\text { Buzzard Ck nr Reagan } \\
\text { Byrds Mill Spring nr Fittstown }\end{array}$ & $\begin{array}{l}\text { STR } \\
\text { CS } \\
\text { QWP } \\
\text { CS } \\
\text { STR }\end{array}$ & $\mathrm{CP}$ & & $\mathbf{R G}$ & $\begin{array}{l}10.7 \\
4.30\end{array}$ & $\begin{array}{l}1986- \\
1965-75 \\
1961 \\
1965-75 \\
1959-\end{array}$ \\
\hline $\begin{array}{l}\emptyset 73342 \emptyset \emptyset \\
\emptyset 72466 \varnothing \emptyset \\
\varnothing 72466 \varnothing \emptyset \\
673365 \varnothing \emptyset \\
\varnothing 733656 \varnothing\end{array}$ & $\begin{array}{l}\text { Byrds Mill Spring nr Fittstown } \\
\text { Cache Ck nr Cowl ington } \\
\text { Cache Ck nr Cowlington } \\
\text { Caddo Ck nr Ardmore } \\
\text { Caddo Ck nr Ardmore }\end{array}$ & $\begin{array}{l}\text { QWP } \\
\text { CS } \\
\text { QWP } \\
\text { STR } \\
\text { QWP }\end{array}$ & $\begin{array}{l}\mathrm{CP} \\
\mathrm{CP}\end{array}$ & & & $\begin{array}{l}20.6 \\
20.6 \\
298 . \\
298 .\end{array}$ & $\begin{array}{l}1953,65-66 \\
1964-72 \\
1959-61 \\
1936-5 \emptyset \\
1950-51,68\end{array}$ \\
\hline $\begin{array}{l}\emptyset 717168 \emptyset \\
\varnothing 722896 \emptyset \\
\varnothing 72286 \emptyset \emptyset \\
\emptyset 72285 \emptyset \emptyset \\
\varnothing 72285 \varnothing \emptyset\end{array}$ & $\begin{array}{l}\text { California Ck nr Nowata } \\
\text { Canadian } R \text { Trib nr Newcastle } \\
\text { Canadian } R \text { at Bridgeport } \\
\text { Canadian } R \text { at Bridgeport } \\
\text { Canadian } R \text { at Bridgeport }\end{array}$ & $\begin{array}{l}\text { QWP } \\
\text { CS } \\
\text { STR } \\
\text { QWD } \\
\text { QWMP }\end{array}$ & $\begin{array}{l}\text { CP } \\
\text { CNP } \\
\text { CNP }\end{array}$ & OBS & JG & $\begin{array}{l}\quad 3.32 \\
26229 . \\
26229 . \\
26229 .\end{array}$ & $\begin{array}{l}1952-53,69 \\
1965-75 \\
1944-64,69- \\
1949-61,64,76-81 \\
1973-79\end{array}$ \\
\hline $\begin{array}{l}\emptyset 72286 \emptyset \emptyset \\
\varnothing 72285 \emptyset \emptyset \\
\varnothing 72315 \emptyset \emptyset \\
\varnothing 72315 \varnothing \emptyset \\
\varnothing 72315 \emptyset \emptyset\end{array}$ & $\begin{array}{l}\text { Canadian } R \text { at Bridgeport } \\
\text { Canadian } R \text { at Bridgeport } \\
\text { Canadian } R \text { at Calvin } \\
\text { Canadian } R \text { at Calvin } \\
\text { Canadian } R \text { at Calvin }\end{array}$ & $\begin{array}{l}\text { SED } \\
\text { QWP } \\
\text { STR } \\
\text { QWP } \\
\text { QWMP }\end{array}$ & $\begin{array}{l}\text { S } \\
\text { CNOPS } \\
\text { BCNP } \\
\text { CNP }\end{array}$ & DCP & $\begin{array}{l}\text { JG } \\
C \\
J G A E\end{array}$ & $\begin{array}{l}26229 \text {. } \\
26229 \\
27952 \\
27952 \\
27952\end{array}$ & $\begin{array}{l}1978-81 \\
1981- \\
* * 1965-66,44- \\
196 \varnothing-63,66-61,65- \\
1973-77\end{array}$ \\
\hline $\begin{array}{l}\emptyset 72315 \varnothing \emptyset \\
\varnothing 723965 \emptyset \\
\varnothing 724496 \emptyset \\
\varnothing 722965 \emptyset \\
\varnothing 72292 \varnothing \emptyset\end{array}$ & $\begin{array}{l}\text { Canadian } R \text { at Calvin } \\
\text { Canadian } R \text { at Cemetary Rd nr Yukon } \\
\text { Canadian } R \text { at L Eufaula TW } \\
\text { Canadian } R \text { at Norman } \\
\text { Canadian } R \text { at Purcell }\end{array}$ & $\begin{array}{l}\text { SED } \\
\text { QWP } \\
\text { RESU } \\
\text { QWP } \\
\text { STR }\end{array}$ & $\begin{array}{l}\mathrm{S} \\
\text { CNOP } \\
\mathrm{CP}\end{array}$ & & $\begin{array}{l}\text { CAE } \\
\text { JG }\end{array}$ & 25939 & $\begin{array}{l}1975-85 \\
1973 \\
1986- \\
1968 \\
1960-61,79-82,86-\end{array}$ \\
\hline $\begin{array}{l}\emptyset 72292 \varnothing \emptyset \\
\varnothing 72292 \varnothing \emptyset \\
\varnothing 72292 \varnothing \emptyset \\
\varnothing 722825 \emptyset \\
\emptyset 7228 \varnothing \emptyset \emptyset\end{array}$ & $\begin{array}{l}\text { Canadian } R \text { at Purcell } \\
\text { Canadian } R \text { at Purcell } \\
\text { Canadian } R \text { at Purcell } \\
\text { Canadian } R \text { at Taloga } \\
\text { Canadian } R \text { nr Canadian TX }\end{array}$ & $\begin{array}{l}\text { QWD } \\
\text { QWMP } \\
\text { SED } \\
\text { QWP } \\
\text { QWMP }\end{array}$ & $\begin{array}{l}\text { CNP } \\
\text { CNP } \\
\text { S } \\
\text { C } \\
\text { CNP }\end{array}$ & & & $\begin{array}{l}25939 . \\
25939 . \\
25939 \text {. } \\
22866 \text {. }\end{array}$ & $\begin{array}{l}1962, * * 60-63,74-76 \\
1975-79 \\
1979-82 \\
1953 \\
1973-77\end{array}$ \\
\hline $\begin{array}{l}\emptyset 7229 \varnothing \emptyset \emptyset \\
\varnothing 72291 \varnothing \emptyset \\
\varnothing 72291 \varnothing \emptyset \\
\emptyset 72291 \varnothing \emptyset \\
\varnothing 72291 \varnothing \emptyset\end{array}$ & $\begin{array}{l}\text { Canadian } R n r \text { Newcastle } \\
\text { Canadian } R n r \text { Noble } \\
\text { Canadian } R n r \text { Noble } \\
\text { Canadian } R n r \text { Noble } \\
\text { Canadian } R n r \text { Noble }\end{array}$ & $\begin{array}{l}\text { STR } \\
\text { STR } \\
\text { QWD } \\
\text { QWMP } \\
\text { SED }\end{array}$ & $\begin{array}{l}\text { CNP } \\
\text { CNP } \\
S\end{array}$ & & & $\begin{array}{l}25763 . \\
25911 \\
26911 \\
25911 \\
26911\end{array}$ & $\begin{array}{l}1939-46 \\
1960-61,63-75 \\
1966-71,73-74 \\
1973-76 \\
1976\end{array}$ \\
\hline
\end{tabular}

* Continuous streamflow records for this period.

* Some records in intervening years.

*** Some records may have been collected previously. 
Tabl- 1.--Alphabetical listing of current and historical gaging stations maintained by the

U.S. Geological Survey

September 90, 1987--Continued

[Symbols defined on last page of report]

\begin{tabular}{|c|c|c|c|c|c|c|c|}
\hline $\begin{array}{r}\text { Station } \\
\text { Number }\end{array}$ & Station & $\begin{array}{l}\text { Type } \\
\text { of } \\
\text { Data }\end{array}$ & $\begin{array}{l}\text { Q.W. } \\
\text { Para- } \\
\text { moters }\end{array}$ & $\begin{array}{l}\text { Stage } \\
\text { Source }\end{array}$ & $\begin{array}{l}\text { Coop- } \\
\text { erator }\end{array}$ & $\begin{array}{c}\text { Drainage } \\
\text { Area } \\
(\text { mile })\end{array}$ & Poriod of Record \\
\hline $\begin{array}{l}\varnothing 72282 \varnothing \emptyset \\
\varnothing 72282 \varnothing \emptyset \\
\varnothing 72283 \varnothing \emptyset \\
\varnothing 72287 \varnothing \emptyset \\
\varnothing 7246 \varnothing \varnothing \emptyset\end{array}$ & $\begin{array}{l}\text { Canadian } R n r \text { Roll } \\
\text { Canadian } R n r \text { Roll } \\
\text { Canadian } R n r \text { Thomas } \\
\text { Canadian } R n r \text { Union City } \\
\text { Canadian } R n r \text { Whitofield }\end{array}$ & $\begin{array}{l}\text { QWP } \\
\text { QWMP } \\
\text { QWP } \\
\text { QWP } \\
\text { STR }\end{array}$ & $\begin{array}{l}\text { CP } \\
\text { CNP } \\
\text { CP } \\
\text { CNP }\end{array}$ & DCP & $c$ & $\begin{array}{l}23616 \\
23615\end{array}$ & $\begin{array}{l}1950-53,62-63 \\
1950-51,76-77 \\
1952-53,61-63 \\
1962-53,73 \\
1938-\end{array}$ \\
\hline $\begin{array}{l}\varnothing 7246 \varnothing \varnothing \varnothing \\
\varnothing 7246 \varnothing \varnothing \emptyset \\
\varnothing 7246 \varnothing \varnothing \emptyset \\
\varnothing 7246 \varnothing \varnothing \emptyset \\
\varnothing 7229427\end{array}$ & $\begin{array}{l}\text { Canadian } R n r \text { Whitofield } \\
\text { Canadian } R n r \text { Whitofiold } \\
\text { Canadian } R n r \text { Whitefield } \\
\text { Canadian } R n r \text { Whitofiold } \\
\text { Canadian Sandy Ck nr Ada }\end{array}$ & $\begin{array}{l}\text { QWP } \\
\text { QWMP } \\
\text { SED } \\
\text { QWD } \\
\text { STR }\end{array}$ & $\begin{array}{l}\text { BCNP } \\
\text { CNP } \\
S \\
P\end{array}$ & OBS & $\begin{array}{l}J G A \\
\text { JGA } \\
B\end{array}$ & $\begin{array}{l}47576 \\
47576 \\
47576 \\
47576\end{array}$ & $\begin{array}{l}1947-64,67-86 \\
1973-77 \\
1976- \\
1944-46,46-64,66-86 \\
1986-\end{array}$ \\
\hline $\begin{array}{l}\emptyset 7229427 \\
\varnothing 7229427 \\
\varnothing 7176525 \\
\varnothing 717686 \emptyset \\
\varnothing 7166581\end{array}$ & $\begin{array}{l}\text { Canadian Sandy Ck nr Ada } \\
\text { Canadian Sandy Ck nr Ada } \\
\text { Candy Ck } n r \text { Avant } \\
\text { Candy Ck nr Wolco } \\
\text { Cane Ck nr Jamesville }\end{array}$ & $\begin{array}{l}\text { QWP } \\
\text { SED } \\
\text { QWP } \\
\text { STRP } \\
\text { QWP }\end{array}$ & $\begin{array}{l}C \\
S \\
C P \\
C P\end{array}$ & $\mathbf{N}$ & $\begin{array}{l}P \\
B\end{array}$ & 30.6 & $\begin{array}{l}1986- \\
1987- \\
1952,65-66 \\
1969-81 \\
1960-61\end{array}$ \\
\hline $\begin{array}{l}\varnothing 73328 \varnothing \emptyset \\
\varnothing 71746 \varnothing \emptyset \\
\varnothing 71746 \varnothing \emptyset \\
\varnothing 71746 \varnothing \emptyset \\
\varnothing 71746 \varnothing \emptyset\end{array}$ & $\begin{array}{l}\text { Caney Boggy Ck nr Ashland } \\
\text { Caney Ck nr Copan } \\
\text { Caney } R \text { at Bartlesville } \\
\text { Caney } R \text { at Bartlesville } \\
\text { Caney } R \text { at Bartlesville }\end{array}$ & $\begin{array}{l}\text { QWD } \\
\text { STRP } \\
\text { STRP } \\
\text { QWP } \\
\text { STR }\end{array}$ & CNP & DCP & C & $\begin{array}{l}49.0 \\
424 . \\
1465 \\
1465 \\
1465\end{array}$ & $\begin{array}{l}1972-76 \\
1944-58 \\
1950-56 \\
1952-53,67-68 \\
1950-56,86-\end{array}$ \\
\hline $\begin{array}{l}\varnothing 717247 \varnothing \\
\varnothing 717555 \emptyset \\
\varnothing 717555 \varnothing \\
\varnothing 717555 \varnothing \\
\varnothing 717206 \varnothing\end{array}$ & $\begin{array}{l}\text { Caney R nr Boulangerville } \\
\text { Caney R nr Collinsville } \\
\text { Caney R nr Collinsville } \\
\text { Caney R nr Collinsville } \\
\text { Caney R nr Elgin KS }\end{array}$ & $\begin{array}{l}\text { QWP } \\
\text { QWP } \\
\text { LF } \\
\text { STGU } \\
\text { SED }\end{array}$ & $\begin{array}{l}C P \\
C P\end{array}$ & DCP & $\begin{array}{l}2 G \\
C\end{array}$ & 445 . & $\begin{array}{l}1952-53 \\
1949-53,69 \\
1979-84 \\
1986- \\
1973-75\end{array}$ \\
\hline $\begin{array}{l}\varnothing 717306 \varnothing \\
\varnothing 717306 \varnothing \\
\varnothing 717306 \varnothing \\
\varnothing 71747 \varnothing \varnothing \\
\varnothing 71747 \varnothing \varnothing\end{array}$ & $\begin{array}{l}\text { Caney R nr Hulah } \\
\text { Caney R nr Hulah } \\
\text { Caney R nr Hulah } \\
\text { Caney R nr Ochelata } \\
\text { Caney R nr Ochelata }\end{array}$ & $\begin{array}{l}\text { STR } \\
\text { QWP } \\
\text { QWMP } \\
\text { STRP } \\
\text { QWP }\end{array}$ & $\begin{array}{l}C P \\
C N P \\
C P\end{array}$ & & $\mathrm{C}$ & $\begin{array}{l}736 \\
736 \\
736 \\
1753 \\
1753\end{array}$ & $\begin{array}{l}1937- \\
1952-63, * *, 60,63-64 \\
1975-79 \\
1956-76 \\
1960-61\end{array}$ \\
\hline $\begin{array}{l}\varnothing 71747 \varnothing \varnothing \\
\varnothing 71756 \varnothing \emptyset \\
\varnothing 71756 \varnothing \varnothing \\
\varnothing 71756 \varnothing \emptyset \\
\varnothing 72385 \varnothing \varnothing\end{array}$ & $\begin{array}{l}\text { Caney } R \mathrm{nr} \text { Ochelata } \\
\text { Caney } R \mathrm{nr} \text { Ramona } \\
\text { Caney } R \mathrm{nr} \text { Ramona } \\
\text { Caney } R \mathrm{nr} \text { Ramona } \\
\text { Canton Lake nr Canton }\end{array}$ & $\begin{array}{l}\text { QWMP } \\
\text { STR } \\
\text { QWP } \\
\text { QWMP } \\
\text { RES }\end{array}$ & $\begin{array}{l}\text { CNP } \\
\text { CNP } \\
\text { CNP }\end{array}$ & DCP & $\begin{array}{l}\mathrm{C} \\
\mathrm{JG} \\
\mathrm{C}\end{array}$ & $\begin{array}{l}1753 \\
1955 \\
1955 \\
1956 \\
12483\end{array}$ & $\begin{array}{l}1973-75 \\
1935-391935-3945- \\
1952-53, * * 65- \\
1975-79 \\
1948-\end{array}$ \\
\hline $\begin{array}{l}\emptyset 72386 \varnothing \emptyset \\
\varnothing 736948 \emptyset \\
\varnothing 72286 \varnothing \emptyset \\
\varnothing 71544 \varnothing \emptyset \\
\varnothing 72486 \varnothing \emptyset\end{array}$ & $\begin{array}{l}\text { Canton Lake nr Canton } \\
\text { Canyon Ck nr Medicine Park } \\
\text { Canyon View Ck nr Geary } \\
\text { Carrizozo Ck nr Kenton } \\
\text { Caston Ck at Wister }\end{array}$ & $\begin{array}{l}\text { QWP } \\
\text { CS } \\
\text { CS } \\
\text { CS } \\
\text { STR }\end{array}$ & $\mathrm{CP}$ & & JG & $\begin{array}{c}12483 . \\
3.35 \\
11.8 \\
111 . \\
72.9\end{array}$ & $\begin{array}{l}* * 1949-50,68- \\
1965-75 \\
1964-72 \\
1953-70 \\
1978-82\end{array}$ \\
\hline
\end{tabular}

* Continuous streamflow records for this period.

* Some records in intervening years.

*** Some records may have been collected previously. 
Table 1.--Alphabetical listing of current and historical gaging stations maintained by the U.S. Geological Survey

September 90, 1987--Continued

[Symbols defined on last page of report]

\begin{tabular}{|c|c|c|c|c|c|c|c|}
\hline $\begin{array}{l}\text { Station } \\
\text { Number }\end{array}$ & Station & $\begin{array}{l}\text { Type } \\
\text { of } \\
\text { Data }\end{array}$ & $\begin{array}{l}\text { Q.W. } \\
\text { Para- } \\
\text { meters }\end{array}$ & $\begin{array}{l}\text { Stage } \\
\text { Source }\end{array}$ & $\begin{array}{l}\text { Coop- } \\
\text { orator }\end{array}$ & $\begin{array}{c}\text { Drainage } \\
\text { Area } \\
\left(m i l e^{2}\right)\end{array}$ & Period of Record \\
\hline $\begin{array}{l}\emptyset 72486 \varnothing \emptyset \\
\varnothing 72486 \varnothing \emptyset \\
\varnothing 733333 \emptyset \\
\varnothing 73335 \varnothing \emptyset \\
\varnothing 733350 \varnothing\end{array}$ & $\begin{array}{l}\text { Caston Ck at Wister } \\
\text { Caston Ck at Wister } \\
\text { Chickasaw Ck Trib nr Stringtown } \\
\text { Chickasaw Ck nr Stringtown } \\
\text { Chickasaw Ck nr Stringtown }\end{array}$ & $\begin{array}{l}\text { QWP } \\
\text { SED } \\
\text { CS } \\
\text { CS } \\
\text { QWP }\end{array}$ & $\begin{array}{l}\text { BCNP } \\
S\end{array}$ & & & $\begin{array}{l}72.9 \\
72.9 \\
3.19 \\
32.7 \\
32.7\end{array}$ & $\begin{array}{l}1978-81 \\
1978-81 \\
1965-72 \\
* 1956-68,69-75 \\
1955-58,6 \varnothing\end{array}$ \\
\hline $\begin{array}{l}\emptyset 7333500 \\
\varnothing 7329772 \\
\emptyset 7151903 \\
\varnothing 7152000 \\
\varnothing 7152000\end{array}$ & $\begin{array}{l}\text { Chickasaw Ck nr Stringtown } \\
\text { Chigley Sandy Ck nr Davis } \\
\text { Chikaskia } R \text { blw Braman Lake nr Braman } \\
\text { Chikaskia } R \text { n Blackwell } \\
\text { Chikaskia } R \text { nr Blackwell }\end{array}$ & $\begin{array}{l}\text { STR } \\
\text { QWP } \\
\text { QWP } \\
\text { STR } \\
\text { QWP }\end{array}$ & $\begin{array}{r}\mathrm{CNP} \\
\mathrm{CP} \\
\mathrm{CP} \\
\mathrm{CP}\end{array}$ & $D C P$ & JGC & $\begin{array}{l}1859 . \\
1859 .\end{array}$ & $\begin{array}{l}1955-68 \\
1955,61 \\
1958,59,62 \\
1935- \\
1952-63\end{array}$ \\
\hline 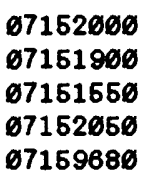 & $\begin{array}{l}\text { Chikaskia } R n r \text { Blackwell } \\
\text { Chikaskia } R \text { nr Braman } \\
\text { Chikaskia } R n r \text { Drury KS } \\
\text { Chikaskia } R \text { nr Tonkawa } \\
\text { Chisholm Ck at Village Dr, OKC }\end{array}$ & $\begin{array}{l}\text { QWMP } \\
\text { QWMP } \\
\text { QWP } \\
\text { QWP } \\
\text { CSR }\end{array}$ & $\begin{array}{l}\text { CNP } \\
\text { CNP } \\
C \\
C P\end{array}$ & & & $\begin{array}{l}1859 . \\
1610 . \\
1.22\end{array}$ & $\begin{array}{l}1975-79 \\
1977 \\
1960-61 \\
1948,51,53,58-63 \\
1976-78\end{array}$ \\
\hline $\begin{array}{l}\emptyset 717867 \varnothing \\
\varnothing 717867 \varnothing \\
\varnothing 7178646 \\
\varnothing 733247 \varnothing \\
\varnothing 715812 \varnothing\end{array}$ & $\begin{array}{l}\text { Chouteau L D nr Chouteau (DS) } \\
\text { Chouteau L D nr Chouteau (DS) } \\
\text { Chouteau L D nr Chouteau (US) } \\
\text { Chuckwa Ck nr Durant } \\
\text { Cimarron R Trib nr Isabel la }\end{array}$ & $\begin{array}{l}\text { QWP } \\
\text { STGU } \\
\text { STGU } \\
\text { QWP } \\
\text { CS }\end{array}$ & $\mathrm{CP}$ & $D C P$ & $\begin{array}{l}c \\
c\end{array}$ & 0.62 & $\begin{array}{l}1952-53,60-63 \\
1986- \\
1986- \\
1953 \\
1964-72\end{array}$ \\
\hline $\begin{array}{l}\varnothing 715802 \emptyset \\
\varnothing 71550 \varnothing \varnothing \\
\varnothing 715798 \varnothing \\
\varnothing 715798 \varnothing \\
\varnothing 715798 \varnothing\end{array}$ & $\begin{array}{l}\text { Cimarron } R \text { Trib nr Lone Wolf } \\
\text { Cimarron } R \text { abv Ute Ck nr Boise City } \\
\text { Cimarron } R \text { at Freedom } \\
\text { Cimarron } R \text { at Freedom } \\
\text { Cimarron } R \text { at Freedom }\end{array}$ & $\begin{array}{l}\text { CS } \\
\text { STR } \\
\text { STR } \\
\text { QWH } \\
\text { QWMP }\end{array}$ & $\begin{array}{l}\text { CP } \\
\text { CNP }\end{array}$ & & & $\begin{array}{l}4.07 \\
1955 . \\
127 ø 6 . \\
12706 \\
12706\end{array}$ & $\begin{array}{l}1964-75 \\
1965-67,43-54 \\
1973-80 \\
195374-80 \\
1976-77\end{array}$ \\
\hline $\begin{array}{l}07164000 \\
07164000 \\
\emptyset 7163500 \\
\emptyset 7163500 \\
67163500\end{array}$ & $\begin{array}{l}\text { Cimarron } R \text { at Mannford } \\
\text { Cimarron } R \text { at Mannford } \\
\text { Cimarron } R \text { at } 0 i l \text { ton } \\
\text { Cimarron } R \text { at } 0 i l \text { ton } \\
\text { Cimarron } R \text { at } 0 i l t o n\end{array}$ & $\begin{array}{l}\text { STR } \\
\text { QWP } \\
\text { CS } \\
\text { STR } \\
\text { QWP }\end{array}$ & $\mathrm{CP}$ & & & $\begin{array}{l}18849 \\
18849 \\
18669 \\
18669 \\
18669\end{array}$ & $\begin{array}{l}1939-50,59-63 \\
1950-62,60-63 \\
1964-75 \\
1934-45 \\
1944\end{array}$ \\
\hline $\begin{array}{l}\varnothing 7161000 \\
\varnothing 7161000 \\
\varnothing 7161000 \\
\varnothing 7161000 \\
\varnothing 7161000\end{array}$ & $\begin{array}{l}\text { Cimarron } R \text { at Perkins } \\
\text { Cimarron } R \text { at Perkins } \\
\text { Cimarron } R \text { at Perkins } \\
\text { Cimarron } R \text { at Perkins } \\
\text { Cimarron } R \text { at Perkins }\end{array}$ & $\begin{array}{l}\text { STR } \\
\text { QWH } \\
\text { QWMP } \\
\text { SED } \\
\text { QWP }\end{array}$ & $\begin{array}{l}\text { BCNP } \\
\text { CNP } \\
S \\
\text { BCNP }\end{array}$ & $D C P$ & $\begin{array}{l}\text { CAE } \\
\text { AE }\end{array}$ & $\begin{array}{l}17852 \\
17852 \\
17852 \\
17852 \\
17852\end{array}$ & $\begin{array}{l}1939- \\
1959,53-63,69-80 \\
1973-77 \\
* * * 1973- \\
1981-\end{array}$ \\
\hline $\begin{array}{l}\emptyset 715650 \emptyset \\
\emptyset 715795 \emptyset \\
\varnothing 715795 \emptyset \\
\varnothing 715795 \emptyset \\
\varnothing 715795 \varnothing\end{array}$ & $\begin{array}{l}\text { Cimarron } R \text { nr Boise City } \\
\text { Cimarron R nr Buffalo } \\
\text { Cimarron R nr Buffalo } \\
\text { Cimarron R nr Buffalo } \\
\text { Cimarron } R \text { nr Buffalo }\end{array}$ & $\begin{array}{l}\text { STR } \\
\text { STR } \\
\text { QWD } \\
\text { QWMP } \\
\text { SED }\end{array}$ & $\begin{array}{l}\text { BCNP } \\
\text { CNP } \\
S\end{array}$ & OBS & A & $\begin{array}{l}2214 \\
12004 \\
12004 \\
12004 \\
12004\end{array}$ & $\begin{array}{l}1939-42 \\
1960- \\
1953,60-63,68-79 \\
1973-77 \\
* * * 1973-\end{array}$ \\
\hline
\end{tabular}

* Continuous streamflow records for this period.

** Some records in intervening years.

*** Some records may have been collected previously. 
Table 1.--Alphabetical listing of current and historical gaging stations maintained by the U.S. Geological Survey

September 90, 1987--Continued

[Symbols defined on last page of report]

\begin{tabular}{|c|c|c|c|c|c|c|c|}
\hline $\begin{array}{r}\text { Station } \\
\text { Number }\end{array}$ & Station & $\begin{array}{l}\text { Typo } \\
\text { of } \\
\text { Data }\end{array}$ & $\begin{array}{l}\text { Q.W. } \\
\text { Para- } \\
\text { moters }\end{array}$ & $\begin{array}{l}\text { Stage } \\
\text { Source }\end{array}$ & $\begin{array}{l}\text { Coop- } \\
\text { orator }\end{array}$ & $\begin{array}{c}\text { Drainago } \\
\text { Areag } \\
\left(\mathrm{mi} \mid \mathrm{l}^{2}\right)\end{array}$ & Period of Record \\
\hline 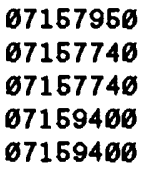 & $\begin{array}{l}\text { Cimarron } R \text { nr Buffalo } \\
\text { Cimarron } R \text { nr Buttermilk KS } \\
\text { Cimarron R nr Buttermilk KS } \\
\text { Cimarron R nr Crescont } \\
\text { Cimarron R nr Crescont }\end{array}$ & $\begin{array}{l}\text { QWP } \\
\text { STR } \\
\text { QWH } \\
\text { STR } \\
\text { QWP }\end{array}$ & $\begin{array}{l}\mathrm{BCNP} \\
\mathrm{CP} \\
\mathrm{CP}\end{array}$ & & $J G A$ & $\begin{array}{l}12004 \\
11120 \\
11120 \\
16453 \\
16463\end{array}$ & $\begin{array}{l}1980- \\
1973-79 \\
1973-79 \\
1970-72 \\
1953\end{array}$ \\
\hline $\begin{array}{l}\sigma 715916 \emptyset \\
6715916 \emptyset \\
\sigma 715916 \emptyset \\
6715758 \emptyset \\
\sigma 716758 \emptyset\end{array}$ & $\begin{array}{l}\text { Cimarron } R n r \text { Dover } \\
\text { Cimarron } R n r \text { Dover } \\
\text { Cimarron R } n r \text { Dover } \\
\text { Cimarron } R \mathrm{nr} \text { Enlgewood KS } \\
\text { Cimarron R nr Enlgewood KS }\end{array}$ & $\begin{array}{l}\text { STR } \\
\text { QWH } \\
\text { QWMP } \\
\text { STR } \\
\text { QWP }\end{array}$ & $\begin{array}{l}\text { CP } \\
\text { CNP } \\
\text { CNP }\end{array}$ & DCP & $\begin{array}{l}J G \\
J G\end{array}$ & $\begin{array}{l}16713 \\
15713 \\
15713 \\
10096 \\
10096\end{array}$ & $\begin{array}{l}1973- \\
1951,53,74-79 \\
1975-79 \\
1982-87 \\
1982-87\end{array}$ \\
\hline $\begin{array}{l}\emptyset 715758 \emptyset \\
671569 \varnothing \emptyset \\
\varnothing 716 \varnothing \varnothing \varnothing \emptyset \\
6716 \varnothing \varnothing \varnothing \emptyset \\
\varnothing 716 \varnothing \varnothing \varnothing \emptyset\end{array}$ & $\begin{array}{l}\text { Cimarron } R \text { nr Enlgowood KS } \\
\text { Cimarron } R \text { nr Forgan KS } \\
\text { Cimarron } R \text { nr Guthrie } \\
\text { Cimarron } R \text { nr Guthrie } \\
\text { Cimarron } R \text { nr Guthrie }\end{array}$ & $\begin{array}{l}\text { SED } \\
\text { STR } \\
\text { STR } \\
\text { QWD } \\
\text { QWMP }\end{array}$ & $\begin{array}{l}\text { CP } \\
\text { CNP }\end{array}$ & & $\begin{array}{l}J G \\
J G \\
J G\end{array}$ & $\begin{array}{r}10696 \\
8536 \\
16892 \\
16892 \\
16892\end{array}$ & $\begin{array}{l}1982-87 \\
1965- \\
1949,63-63,83- \\
1949,63-63 \\
1973-79\end{array}$ \\
\hline $\begin{array}{l}\emptyset 716 \varnothing \emptyset \emptyset \emptyset \\
671545 \varnothing \emptyset \\
\varnothing 71546 \varnothing \emptyset \\
671546 \varnothing \emptyset \\
671546 \varnothing \emptyset\end{array}$ & $\begin{array}{l}\text { Cimarron } R n r \text { Guthrie } \\
\text { Cimarron } R n r \text { Kenton } \\
\text { Cimarron } R n r \text { Kenton } \\
\text { Cimarron } R n r \text { Kenton } \\
\text { Cimarron } R n r \text { Kenton }\end{array}$ & $\begin{array}{l}\text { SED } \\
\text { STR } \\
\text { QWP } \\
\text { QWMP } \\
\text { QWP }\end{array}$ & $\begin{array}{l}S \\
\text { CP } \\
\text { CNP } \\
\text { CNP }\end{array}$ & & B & $\begin{array}{r}16892 \\
1166 \\
1166 \\
1166 \\
1166\end{array}$ & $\begin{array}{l}1976 \\
1950- \\
1952-63 \\
1977 \\
1987-\end{array}$ \\
\hline 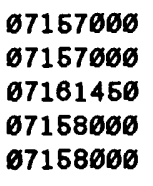 & $\begin{array}{l}\text { Cimarron } R \text { nr Mocane } \\
\text { Cimarron } R \mathrm{nr} \text { Mocane } \\
\text { Cimarron } R \mathrm{nr} R \mathrm{R} \text { ipley } \\
\text { Cimarron } R \mathrm{nr} \text { Waynoka } \\
\text { Cimarron R nr Waynoka }\end{array}$ & $\begin{array}{l}\text { PR } \\
\text { QWP } \\
\text { STR } \\
\text { STR } \\
\text { QWH }\end{array}$ & $\mathrm{CP}$ & DCP & $\begin{array}{l}\mathrm{C} \\
\mathrm{C}\end{array}$ & $\begin{array}{l}867 \varnothing . \\
8670 . \\
13334 \\
13334\end{array}$ & $\begin{array}{l}* 1943-65,76-79 \\
1947-49,52-64,76-79 \\
1987- \\
1937- \\
1961-63,68-79\end{array}$ \\
\hline $\begin{array}{l}\varnothing 7158 \varnothing \varnothing \varnothing \\
\varnothing 73348 \varnothing \emptyset \\
\varnothing 73356 \varnothing \emptyset \\
\varnothing 73356 \varnothing \emptyset \\
\varnothing 7335 \varnothing \varnothing \emptyset\end{array}$ & $\begin{array}{l}\text { Cimarron R nr Waynoka } \\
\text { Clear Boggy Ck abv Caney Ck nr Caney } \\
\text { Clear Boggy Ck nr Caney } \\
\text { Clear Boggy Ck nr Caney } \\
\text { Clear Boggy Ck nr Caney }\end{array}$ & $\begin{array}{l}\text { QWMP } \\
\text { QWMP } \\
\text { STRP } \\
\text { QWP } \\
\text { QWMP }\end{array}$ & $\begin{array}{l}\text { CP } \\
\text { CNP }\end{array}$ & & $\mathrm{C}$ & $\begin{array}{l}13334 . \\
720 \\
720 \\
720\end{array}$ & $\begin{array}{l}1973-77 \\
1975-78 \\
1942- \\
1952-75 \\
1975-79\end{array}$ \\
\hline $\begin{array}{l}\emptyset 73344 \emptyset \emptyset \\
\varnothing 73344 \varnothing \emptyset \\
\varnothing 73345 \varnothing \emptyset \\
\varnothing 723429 \emptyset \\
\varnothing 717112 \emptyset\end{array}$ & $\begin{array}{l}\text { Clear Boggy Ck nr Tupelo } \\
\text { Clear Boggy Ck nr Tupelo } \\
\text { Clear Boggy Ck nr Wapanucka } \\
\text { Clear Ck Trib nr Catosby } \\
\text { Clear Ck Trib nr Hollow }\end{array}$ & $\begin{array}{l}\text { LF } \\
\text { QWP } \\
\text { STR } \\
\text { CS } \\
\text { CS }\end{array}$ & COP & & $\begin{array}{l}H G \\
H J G\end{array}$ & $\begin{array}{l}248 . \\
248 \\
516 \\
8.51 \\
2.19\end{array}$ & $\begin{array}{l}1958-73 \\
1958,60,62 \\
194 \emptyset-43 \\
1966-85 \\
1966-75,79-85\end{array}$ \\
\hline $\begin{array}{l}\emptyset 72341 \varnothing \emptyset \\
\varnothing 723416 \emptyset \\
\emptyset 72343 \varnothing \emptyset \\
\varnothing 72296 \varnothing 1 \\
\varnothing 7249415\end{array}$ & $\begin{array}{l}\text { Clear Ck nr Elmwood } \\
\text { Clear Ck nr Elmwood } \\
\text { Clear Ck nr May } \\
\text { Clear Ck nr Norman } \\
\text { Coal Ck Trib nr Bokoshe }\end{array}$ & $\begin{array}{l}\text { STR } \\
\text { QWP } \\
\text { QWP } \\
\text { QWP } \\
\text { PR }\end{array}$ & $\begin{array}{l}C P \\
C P \\
C P\end{array}$ & & $J G$ & $\begin{array}{l}170 . \\
170 \\
169 . \\
1.26\end{array}$ & $\begin{array}{l}1965- \\
1952-53 \\
1954-58,60 \\
1961 \\
1976-79\end{array}$ \\
\hline
\end{tabular}

* Continuous streamflow records for this poriod.

** Some records in intervening years.

*** Some records may have been collected previously. 
Table 1.--Alphabetical listing of current and historical gaging stations maintained by the U.S. Geological Survey

September 30, 1987--Continued

[Symbols defined on last page of report]

\begin{tabular}{|c|c|c|c|c|c|c|c|}
\hline $\begin{array}{r}\text { Station } \\
\text { Number }\end{array}$ & Station & $\begin{array}{l}\text { Type } \\
\text { of } \\
\text { Data }\end{array}$ & $\begin{array}{l}\text { Q.W. } \\
\text { Para- } \\
\text { meters }\end{array}$ & $\begin{array}{l}\text { Stage } \\
\text { Source }\end{array}$ & $\begin{array}{l}\text { Coop- } \\
\text { erator }\end{array}$ & $\begin{array}{c}\text { Drainage } \\
\text { Area } \\
\left(m i e^{2}\right)\end{array}$ & Period of Record \\
\hline $\begin{array}{l}\varnothing 7249416 \\
\varnothing 7249416 \\
\varnothing 73329 \varnothing \emptyset \\
\varnothing 73329 \varnothing \emptyset \\
\varnothing 73329 \varnothing \emptyset\end{array}$ & $\begin{array}{l}\text { Coal Ck Trib nr Bokoshe } \\
\text { Coal Ck Trib nr Bokoshe } \\
\text { Coal Ck nr Lehigh } \\
\text { Coal Ck nr Lehigh } \\
\text { Coal Ck nr Lehigh }\end{array}$ & $\begin{array}{l}\text { QWP } \\
\text { SED } \\
\text { STR } \\
\text { QWH } \\
\text { SED }\end{array}$ & $\begin{array}{l}\text { CNP } \\
\mathrm{S} \\
\text { CNOP } \\
\mathrm{S}\end{array}$ & & & $\begin{array}{l}1.26 \\
1.26 \\
8.10 \\
8.10 \\
8.10\end{array}$ & $\begin{array}{l}1976-79 \\
1976-79 \\
1978-81 \\
1977-81 \\
1978-81\end{array}$ \\
\hline $\begin{array}{l}\emptyset 7232627 \\
\emptyset 7246615 \\
\varnothing 7246615 \\
\varnothing 7246615 \\
\varnothing 7246615\end{array}$ & $\begin{array}{l}\text { Coal Ck nr McAlester } \\
\text { Coal Ck nr Spiro } \\
\text { Coal Ck } n r \text { Spiro } \\
\text { Coal Ck nr Spiro } \\
\text { Coal Ck nr Spiro }\end{array}$ & $\begin{array}{l}\text { QWP } \\
\text { STR } \\
\text { QWH } \\
\text { SED } \\
\text { QWP }\end{array}$ & $\begin{array}{l}\text { CP } \\
P \\
S \\
\text { BCNP }\end{array}$ & & & $\begin{array}{r}196 . \\
18.1 \\
18.1 \\
18.1 \\
18.1\end{array}$ & $\begin{array}{l}1960-61 \\
1978-82 \\
1978-82 \\
1978-81 \\
1978-81\end{array}$ \\
\hline $\begin{array}{l}\emptyset 73258 \varnothing \emptyset \\
\varnothing 7326 \varnothing \emptyset \emptyset \\
\varnothing 7326 \varnothing \emptyset \emptyset \\
\varnothing 71652 \varnothing \emptyset \\
\varnothing 71551 \varnothing \emptyset\end{array}$ & $\begin{array}{l}\text { Cobb Ck nr Eakly } \\
\text { Cobb Ck nr Ft Cobb } \\
\text { Cobb Ck nr Ft Cobb } \\
\text { Cold Springs Ck nr Castaneda } \\
\text { Cold Springs Ck nr Wheeless }\end{array}$ & $\begin{array}{l}\text { STR } \\
\text { QWP } \\
\text { STR } \\
\text { LF } \\
\text { CS }\end{array}$ & $\mathrm{CP}$ & & $\begin{array}{l}\text { NG } \\
\text { NG } \\
\text { HG }\end{array}$ & $\begin{array}{l}132 . \\
313 \\
313 . \\
129 . \\
11 .\end{array}$ & $\begin{array}{l}1988- \\
* * 1947-58,60,63 \\
1939- \\
1965-71 \\
1964-85\end{array}$ \\
\hline $\begin{array}{l}\varnothing 723295 \varnothing \\
\varnothing 7233 \varnothing \varnothing \emptyset \\
\varnothing 7233 \varnothing \varnothing \emptyset \\
\varnothing 722815 \emptyset \\
\varnothing 717451 \emptyset\end{array}$ & $\begin{array}{l}\text { Coldwater Ck nr Guymon } \\
\text { Coldwater Ck nr Hardesty } \\
\text { Coldwater Ck nr Hardesty } \\
\text { Commission Ck nr Grand } \\
\text { Coon Ck nr Dewey }\end{array}$ & $\begin{array}{l}\text { STR } \\
\text { STR } \\
\text { QWP } \\
\text { LF } \\
\text { QWP }\end{array}$ & $\begin{array}{l}\mathrm{CP} \\
\mathrm{CP}\end{array}$ & & GJ & $\begin{array}{l}1967 . \\
1967 . \\
67.8\end{array}$ & $\begin{array}{l}1981- \\
1939-64 \\
1952-63 \\
1965-66 \\
1952-63\end{array}$ \\
\hline $\begin{array}{l}\varnothing 71743 \varnothing \emptyset \\
\varnothing 7163 \varnothing 2 \emptyset \\
\varnothing 717416 \emptyset \\
\varnothing 717416 \emptyset \\
\varnothing 731668 \emptyset\end{array}$ & $\begin{array}{l}\text { Copan Lake nr Copan } \\
\text { Corral Ck } n r \text { Yale } \\
\text { Cotton Ck } n r \text { Copan } \\
\text { Cotton Ck } n r \text { Copan } \\
\text { Cottonwood Ck Trib nr Loco }\end{array}$ & $\begin{array}{l}\text { RES } \\
\text { CS } \\
\text { QWP } \\
\text { QWMP } \\
\text { CS }\end{array}$ & $\begin{array}{l}C P \\
C N P\end{array}$ & DCP & $H G$ & 1.74 & $\begin{array}{l}1983- \\
1964-65 \\
1962-63,67-68 \\
1973-79 \\
1964-86\end{array}$ \\
\hline $\begin{array}{l}\emptyset 71598 \emptyset 5 \\
\varnothing 715976 \emptyset \\
\varnothing 715975 \emptyset \\
\varnothing 715975 \emptyset \\
\emptyset 715975 \emptyset\end{array}$ & $\begin{array}{l}\text { Cottonwood } \mathrm{Ck} \text { at Guthrie } \\
\text { Cottonwood } \mathrm{Ck} \text { at Seward } \\
\text { Cottonwood } \mathrm{Ck} \text { at Seward } \\
\text { Cottonwood } \mathrm{Ck} \text { at Seward } \\
\text { Cottonwood } \mathrm{Ck} \text { at Seward }\end{array}$ & $\begin{array}{l}\text { PR } \\
\text { STR } \\
\text { QWD } \\
\text { QWMP } \\
\text { SED }\end{array}$ & $\begin{array}{l}\text { CNP } \\
\text { CNP } \\
S\end{array}$ & & & $\begin{array}{l}376 . \\
316 . \\
316 . \\
316 . \\
316\end{array}$ & $\begin{array}{l}1889-56 \\
1973-82 \\
1973-82 \\
1976-79 \\
1978-82\end{array}$ \\
\hline $\begin{array}{l}\emptyset 71598 \varnothing \emptyset \\
\varnothing 71598 \varnothing \emptyset \\
\emptyset 715972 \emptyset \\
\emptyset 716972 \emptyset \\
\emptyset 716972 \varnothing\end{array}$ & $\begin{array}{l}\text { Cottonwood Ck } n r \text { Guthrie } \\
\text { Cottonwood Ck } n r \text { Guthrie } \\
\text { Cottonwood Ck } n r \text { Navina } \\
\text { Cottonwood Ck } n r \text { Navina } \\
\text { Cottonwood Ck } n r \text { Navina }\end{array}$ & $\begin{array}{l}\text { LF } \\
\text { QWP } \\
\text { STR } \\
\text { QWD } \\
\text { SED }\end{array}$ & $\begin{array}{l}\mathrm{CP} \\
\mathrm{COP} \\
\mathrm{S}\end{array}$ & OBS & JG & $\begin{array}{l}366 . \\
366 \\
247 \\
247 \\
247\end{array}$ & $\begin{array}{l}1952-73 \\
1951 \\
1978-80,82- \\
1978-80 \\
1982-\end{array}$ \\
\hline $\begin{array}{l}\emptyset 716972 \varnothing \\
\varnothing 715972 \emptyset \\
\emptyset 723775 \emptyset \\
\emptyset 7163 \varnothing \emptyset \emptyset \\
\emptyset 7163 \varnothing \emptyset \emptyset\end{array}$ & $\begin{array}{l}\text { Cottonwood Ck nr Navina } \\
\text { Cottonwood Ck nr Navina } \\
\text { Cottonwood Ck nr Vici } \\
\text { Council Ck nr Stillwater } \\
\text { Council Ck nr Stillwater }\end{array}$ & $\begin{array}{l}\text { QWP } \\
\text { QWP } \\
\text { CS } \\
\text { STR } \\
\text { QWP }\end{array}$ & $\begin{array}{l}\mathrm{CP} \\
\mathrm{COB}\end{array}$ & & $\begin{array}{l}J G \\
B \\
H G \\
J G\end{array}$ & $\begin{array}{l}247 \\
247 \\
11.6 \\
31 . \varnothing \\
31 . \varnothing\end{array}$ & $\begin{array}{l}1981- \\
1984 \\
1964-86 \\
1934- \\
1944\end{array}$ \\
\hline
\end{tabular}

\footnotetext{
* Continuous streamflow records for this period.

* Some records in intervening years.

*** Some records may have been collected previously.
} 
Table 1.--Alphabetical listing of current and historical gaging stations maintained by the U.S. Geological Survey

September 90 , 1987--Continued

[Symbols defined on last page of report]

\begin{tabular}{|c|c|c|c|c|c|c|c|}
\hline $\begin{array}{l}\text { Station } \\
\text { Number }\end{array}$ & Station & $\begin{array}{l}\text { Type } \\
\text { of } \\
\text { Data }\end{array}$ & $\begin{array}{l}\text { Q.W. } \\
\text { Para- } \\
\text { meters }\end{array}$ & $\begin{array}{l}\text { Stage } \\
\text { Source }\end{array}$ & $\begin{array}{l}\text { Coop- } \\
\text { orator }\end{array}$ & $\begin{array}{c}\text { Drainage } \\
\text { Area } \\
\left(m i l e^{2}\right)\end{array}$ & Period of Record \\
\hline $\begin{array}{l}\emptyset 7163 \varnothing \varnothing \emptyset \\
\varnothing 73136 \varnothing \emptyset \\
\varnothing 73136 \varnothing \emptyset \\
\varnothing 73136 \varnothing \emptyset \\
\varnothing 73136 \varnothing \emptyset\end{array}$ & $\begin{array}{l}\text { Council Ck nr Still water } \\
\text { Cow Ck at Waurika } \\
\text { Cow Ck at Waurika } \\
\text { Cow Ck at Waurika } \\
\text { Cow Ck at Waurika }\end{array}$ & $\begin{array}{l}\text { SED } \\
\text { CS } \\
\text { QWP } \\
\text { QWMP } \\
\text { STR }\end{array}$ & $\begin{array}{l}S \\
C P \\
C N P\end{array}$ & & GJ & $\begin{array}{l}31.0 \\
193 \\
193 \\
193 \\
193\end{array}$ & $\begin{array}{l}1934-37 \\
* 1967-70,71- \\
1960-63,67-70 \\
1978-79 \\
1967-7 \varnothing\end{array}$ \\
\hline $\begin{array}{l}\emptyset 7313533 \\
\emptyset 7166572 \\
\varnothing 7328173 \\
673282 \varnothing \emptyset \\
671575 \varnothing \emptyset\end{array}$ & $\begin{array}{l}\text { Cow Ck nr Comanche } \\
\text { Coweta Ck nr Coweta } \\
\text { Criner Ck nr Criner } \\
\text { Criner Ck nr Payne } \\
\text { Crooked Ck nr Nye KS }\end{array}$ & $\begin{array}{l}\text { QWP } \\
\text { QWP } \\
\text { QWP } \\
\text { QWP } \\
\text { QWP }\end{array}$ & $\begin{array}{l}C P \\
C P \\
C P \\
C P \\
C P\end{array}$ & & & 1167. & $\begin{array}{l}1959,61 \\
1961 \\
1961 \\
1958-60 \\
1947,68-63\end{array}$ \\
\hline $\begin{array}{l}\emptyset 72416 \varnothing 8 \\
\emptyset 731142 \emptyset \\
\emptyset 716494 \emptyset \\
\emptyset 72311 \emptyset 2 \\
\emptyset 7242219\end{array}$ & $\begin{array}{l}\text { Crutcho Ck Tribat Reno Midwest City } \\
\text { Deadman Ck Trib at Manitou } \\
\text { Deep Ck nr Olive } \\
\text { Deep Ck nr Spaulding } \\
\text { Deep Fk Trib at NW } 50 \text {, OKC }\end{array}$ & $\begin{array}{l}\text { CSR } \\
\text { CS } \\
\text { CS } \\
\text { QWP } \\
\text { CSR }\end{array}$ & $\mathrm{CP}$ & & & $\begin{array}{l}1.41 \\
2.67 \\
3.25 \\
2.65\end{array}$ & $\begin{array}{l}1976-81 \\
1966-72 \\
1967-72 \\
1961 \\
1976-81\end{array}$ \\
\hline $\begin{array}{l}\emptyset 724222 \emptyset \\
\emptyset 7242217 \\
\varnothing 72422 \varnothing \emptyset \\
\emptyset 724238 \emptyset \\
\emptyset 724238 \emptyset\end{array}$ & $\begin{array}{l}\text { Deep Fk at Eastern, OKC } \\
\text { Deep Fk at Pennsylvania Ave, OKC } \\
\text { Deep Fk at Portland, OKC } \\
\text { Deep Fk at Warwick } \\
\text { Deep Fk at Warwick }\end{array}$ & $\begin{array}{l}\text { CSR } \\
\text { QWP } \\
\text { CSR } \\
\text { STR } \\
\text { SED }\end{array}$ & $S$ & $D C P$ & $\begin{array}{l}c \\
c\end{array}$ & $\begin{array}{l}28.2 \\
2.98 \\
622 . \\
622 .\end{array}$ & $\begin{array}{l}1976-81 \\
1960-62 \\
1974-81 \\
1984- \\
1984-\end{array}$ \\
\hline $\begin{array}{l}\emptyset 72423 \varnothing \emptyset \\
\varnothing 724235 \emptyset \\
\varnothing 724235 \emptyset \\
\varnothing 724236 \emptyset \\
\varnothing 724235 \emptyset\end{array}$ & $\begin{array}{l}\text { Deep Fk at Witcher } \\
\text { Deep Fk nr Arcadia } \\
\text { Deep Fk nr Arcadia } \\
\text { Deep Fk nr Arcadia } \\
\text { Deep Fk nr Arcadia }\end{array}$ & $\begin{array}{l}\text { QWP } \\
\text { STR } \\
\text { QWP } \\
\text { QWMP } \\
\text { SED }\end{array}$ & $\begin{array}{l}\text { CNOP } \\
\text { CNP } \\
\text { CNP } \\
S\end{array}$ & OBS & $\begin{array}{l}c \\
C \\
c\end{array}$ & $\begin{array}{l}105 . \\
165 . \\
165 . \\
105 .\end{array}$ & $\begin{array}{l}1968-62,73 \\
1969-87 \\
197 \varnothing-87 \\
1973-79 \\
1978-87\end{array}$ \\
\hline $\begin{array}{l}\emptyset 72435 \varnothing \emptyset \\
\emptyset 72436 \varnothing \emptyset \\
\varnothing 72436 \varnothing \emptyset \\
\varnothing 72435 \varnothing \emptyset \\
\varnothing 72435 \varnothing \emptyset\end{array}$ & $\begin{array}{l}\text { Deep Fk nr Beggs } \\
\text { Deep Fk nr Beggs } \\
\text { Deep Fk } n r \text { Beggs } \\
\text { Deep Fk } n r \text { Beggs } \\
\text { Deep Fk } n r \text { Beggs }\end{array}$ & $\begin{array}{l}\text { STR } \\
\text { QWP } \\
\text { QWMP } \\
\text { SED } \\
\text { QWD }\end{array}$ & $\begin{array}{l}\text { BCNP } \\
\text { CNP } \\
S \\
P\end{array}$ & $D C P$ & $\begin{array}{l}\text { C } \\
\text { JGAE } \\
\text { CAE } \\
\text { JGA }\end{array}$ & $\begin{array}{l}2018 . \\
2018 . \\
2018 . \\
2018 . \\
2018 .\end{array}$ & $\begin{array}{l}1938- \\
1952- \\
1973-79 \\
1978- \\
1951-\end{array}$ \\
\hline 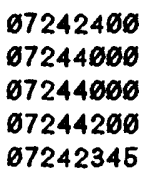 & $\begin{array}{l}\text { Deep Fk nr Chandler } \\
\text { Deep Fk nr Dewar } \\
\text { Deep Fk nr Dewar } \\
\text { Deep Fk nr Pierce } \\
\text { Deep Fork blw Arcadia L nr Arcadia }\end{array}$ & $\begin{array}{l}\text { QWP } \\
\text { STRP } \\
\text { QWD } \\
\text { QWP } \\
\text { STR }\end{array}$ & $\begin{array}{l}C P \\
C P \\
C P \\
Q W P\end{array}$ & & C & $\begin{array}{l}2367 . \\
2307 \\
2548 .\end{array}$ & $\begin{array}{l}196 \emptyset-62 \\
1938-5 \emptyset \\
1949-61 \\
196 \emptyset-63 \\
1987-\end{array}$ \\
\hline $\begin{array}{l}\emptyset 7242346 \\
\emptyset 7242346 \\
\emptyset 73116 \varnothing \emptyset \\
\emptyset 73116 \varnothing \emptyset \\
\varnothing 731150 \emptyset\end{array}$ & $\begin{array}{l}\text { Deep Fork blw Arcadia L nr Arcadia } \\
\text { Deep Fork blw Arcadia L nr Arcadia } \\
\text { Deep Red Run } n r \text { Randlett } \\
\text { Deep Red Run } n r \text { Randlett } \\
\text { Deep Red Run } n r \text { Randlett }\end{array}$ & $\begin{array}{l}\text { QWP } \\
\text { SED } \\
\text { STR } \\
\text { QWD } \\
\text { QWMP }\end{array}$ & $\begin{array}{l}\text { CNP } \\
\text { CP } \\
\text { CNP }\end{array}$ & & $\begin{array}{l}\mathrm{C} \\
\mathrm{C} \\
\mathrm{JG}\end{array}$ & $\begin{array}{l}617 . \\
617 \\
617\end{array}$ & $\begin{array}{l}1987- \\
1987- \\
1949- \\
* * 1948,69-7 \emptyset \\
1975\end{array}$ \\
\hline
\end{tabular}

* Continuous streamflow records for this period.

* Some records in intervening years.

*** Some records may have been collected previously. 
Table 1.--Alphabetical listing of current and historical gaging stations maintained by the U.S. Geological Survey

September s0, 1987--Continued

[Symbols defined on last page of report]

\begin{tabular}{|c|c|c|c|c|c|c|c|}
\hline $\begin{array}{c}\text { Station } \\
\text { Number }\end{array}$ & Station & $\begin{array}{l}\text { Type } \\
\text { of } \\
\text { Data }\end{array}$ & $\begin{array}{l}\text { Q.W. } \\
\text { Para- } \\
\text { meters }\end{array}$ & $\begin{array}{l}\text { Stage } \\
\text { Source }\end{array}$ & $\begin{array}{l}\text { Coop- } \\
\text { orator }\end{array}$ & $\begin{array}{c}\text { Drainage } \\
\text { Area } \\
(\mathrm{mile})\end{array}$ & Period of Record \\
\hline $\begin{array}{l}\emptyset 73116 \varnothing 5 \\
073116 \varnothing 5 \\
\varnothing 722846 \emptyset \\
\emptyset 715963 \emptyset \\
672284 \varnothing \emptyset\end{array}$ & $\begin{array}{l}\text { Deep Red Run nr Taylor } \\
\text { Deep Red Run nr Taylor } \\
\text { Deer Ck Trib nr Hydro } \\
\text { Deer Ck abv Bethany Swg Trtmt PInt *OKC } \\
\text { Deer Ck at Hydro }\end{array}$ & $\begin{array}{l}\text { QWP } \\
\text { QWMP } \\
\text { CS } \\
\text { QWP } \\
\text { STR }\end{array}$ & $\begin{array}{l}\text { CP } \\
\text { CNP } \\
\text { CNOP }\end{array}$ & & & $\begin{array}{l}1121 . \\
1121 \\
27.31 \\
274\end{array}$ & $\begin{array}{l}1959 \\
1976-79 \\
1964-76 \\
1983-84 \\
1961-64,77-8 \emptyset\end{array}$ \\
\hline $\begin{array}{l}\emptyset 72284 \varnothing \emptyset \\
\varnothing 7169646 \\
\varnothing 7232 \varnothing 24 \\
\varnothing 7232 \varnothing 24 \\
\varnothing 7232624\end{array}$ & $\begin{array}{l}\text { Deer Ck at Hydro } \\
\text { Deer Ck blw Deer Ck Swg Trtmt PInt OKC } \\
\text { Deer Ck } n r \text { McAlester } \\
\text { Deer Ck } n r \text { McAlester } \\
\text { Deer Ck nr McAlester }\end{array}$ & $\begin{array}{l}\text { QWP } \\
\text { QWP } \\
\text { STR } \\
\text { QWP } \\
\text { SED }\end{array}$ & $\begin{array}{l}\text { CNP } \\
\text { CNOP } \\
\text { CNP } \\
\text { S }\end{array}$ & & & $\begin{array}{l}274 . \\
38.3 \\
38.3 \\
38.3\end{array}$ & $\begin{array}{l}1951-58,6 \varnothing-63,77-8 \emptyset \\
1983-84 \\
1978-8 \emptyset \\
1978-8 \emptyset \\
1978-8 \emptyset\end{array}$ \\
\hline 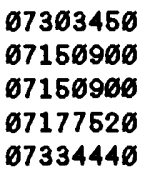 & $\begin{array}{l}\text { Deer Ck nr Plainviow } \\
\text { Deer Ck nr Tonkawa } \\
\text { Deer Ck nr Tonkawa } \\
\text { Delaware Ck nr Sperry } \\
\text { Delaware Ck nr Wapanucka }\end{array}$ & $\begin{array}{l}\text { CSR } \\
\text { LF } \\
\text { QWP } \\
\text { QWP } \\
\text { STR }\end{array}$ & $\begin{array}{l}C P \\
C P\end{array}$ & & & $\begin{array}{l}27.8 \\
160 . \\
160 . \\
45.8\end{array}$ & $\begin{array}{l}1964-77 \\
1965-73 \\
1958-59,62 \\
1952-53 \\
1958-73\end{array}$ \\
\hline $\begin{array}{l}\emptyset 733444 \varnothing \\
\emptyset 731588 \emptyset \\
\varnothing 71985 \varnothing \emptyset \\
\varnothing 71985 \varnothing \emptyset \\
\emptyset 717855 \emptyset\end{array}$ & $\begin{array}{l}\text { Delaware Ck nr Wapanucka } \\
\text { Demijohn Ck nr Wilson } \\
\text { Dirty Ck nr Warner } \\
\text { Dirty Ck nr Warner } \\
\text { Dog Ck nr Claremore }\end{array}$ & $\begin{array}{l}\text { QWP } \\
\text { CS } \\
\text { STRP } \\
\text { QWP } \\
\text { LF }\end{array}$ & $\mathrm{CP}$ & & ZG & $\begin{array}{l}45.8 \\
5.74 \\
227 . \\
227 . \\
63.6\end{array}$ & $\begin{array}{l}1958,6 \emptyset \\
1964-73 \\
194 \varnothing-46 \\
196 \emptyset-61 \\
1979-84\end{array}$ \\
\hline $\begin{array}{l}\emptyset 7175 \varnothing \varnothing \varnothing \\
\varnothing 7175 \varnothing \varnothing \varnothing \\
\varnothing 7313566 \\
\varnothing 7243 \varnothing \varnothing \varnothing \\
\varnothing 7243 \varnothing \varnothing \emptyset\end{array}$ & $\begin{array}{l}\text { Double Ck SWS \#5 nr Ramona } \\
\text { Double Ck SWS \#5 nr Ramona } \\
\text { Dry Ck nr Comanche } \\
\text { Dry Ck nr Kendrick } \\
\text { Dry Ck nr Kendrick }\end{array}$ & $\begin{array}{l}\text { STRP } \\
\text { QWP } \\
\text { QWP } \\
\text { STR } \\
\text { QWP }\end{array}$ & $\begin{array}{l}C P \\
C P \\
C O P\end{array}$ & & $A$ & $\begin{array}{l}2.39 \\
2.39 \\
69.6 \\
69.0\end{array}$ & $\begin{array}{l}1955-69 \\
1954-55, * *, 67-69 \\
1961 \\
1955- \\
* * 1955,70-73\end{array}$ \\
\hline $\begin{array}{l}\varnothing 7367 \varnothing 6 \varnothing \\
\varnothing 717457 \emptyset \\
\varnothing 7162 \varnothing 59 \\
\varnothing 723413 \emptyset \\
\varnothing 732744 \varnothing\end{array}$ & $\begin{array}{l}\text { Dry Fk Otter Ck nr Mountain Park } \\
\text { Dry Hol low nr Pawhuska } \\
\text { Duck Ck nr Tonkawa } \\
\text { Duck Pond } \mathrm{Ck} n r \text { Clear Lake } \\
\text { E Bitter Ck nr Tabler }\end{array}$ & $\begin{array}{l}\text { STR } \\
\text { CS } \\
\text { QWP } \\
\text { LF } \\
\text { QWD }\end{array}$ & $\begin{array}{l}\mathrm{CP} \\
\mathrm{CP}\end{array}$ & & & $\begin{array}{l}12 . \varnothing \\
1.67 \\
97 . \varnothing \\
35.6\end{array}$ & $\begin{array}{l}1995-\varnothing 6 \\
1965-72 \\
1953,62 \\
1966-73 \\
1960-61,68-71\end{array}$ \\
\hline $\begin{array}{l}\emptyset 73225 \varnothing \emptyset \\
\varnothing 73225 \varnothing \emptyset \\
\varnothing 73 \varnothing 889 \emptyset \\
\varnothing 73 \varnothing 9 \varnothing \varnothing \emptyset \\
\varnothing 73 \varnothing 9 \varnothing \varnothing \emptyset\end{array}$ & $\begin{array}{l}\text { E Br Sandstone Ck nr Elk City } \\
\text { E Br Sandstone Ck nr Elk City } \\
\text { E Cache Ck nr Apache } \\
\text { E Cache Ck nr Elgin } \\
\text { E Cache Ck nr Elgin }\end{array}$ & $\begin{array}{l}\text { STR } \\
\text { QWP } \\
\text { QWP } \\
\text { STR } \\
\text { QWD }\end{array}$ & $\begin{array}{l}\mathrm{CP} \\
\mathrm{C} \\
\mathrm{CP}\end{array}$ & & & $\begin{array}{l}23 . \emptyset \\
23 . \emptyset \\
248 . \\
248 .\end{array}$ & $\begin{array}{l}1951-72 \\
1958 \\
1951 \\
1956-58 \\
1956,58\end{array}$ \\
\hline $\begin{array}{l}\varnothing 73 \varnothing 9 \varnothing \varnothing \emptyset \\
\varnothing 7311 \varnothing \varnothing \varnothing \\
\varnothing 7311 \varnothing \varnothing \varnothing \\
\varnothing 7311 \varnothing \varnothing \varnothing \\
\varnothing 73 \varnothing 44 \varnothing 3\end{array}$ & $\begin{array}{l}\text { E Cache Ck nr Elgin } \\
\text { E Cache Ck nr Walters } \\
\text { E Cache Ck nr Walters } \\
\text { E Cache Ck nr Walters } \\
\text { E Elk Ck nr Rocky }\end{array}$ & $\begin{array}{l}\text { QWMP } \\
\text { STR } \\
\text { QWP } \\
\text { QWMP } \\
\text { QWP }\end{array}$ & $\begin{array}{l}\text { CNP } \\
\text { CP } \\
\text { CNP } \\
\text { CP }\end{array}$ & & $\begin{array}{l}\text { UG } \\
\text { UG }\end{array}$ & $\begin{array}{l}248 . \\
675 \\
675 \\
675\end{array}$ & $\begin{array}{l}1975-79 \\
1938-68,69- \\
* * 1947-48,58-63,70- \\
1975-79 \\
1961\end{array}$ \\
\hline
\end{tabular}

* Continuous streamflow records for this period.

* Some records in intervening years.

*** Some records may have been collected previously. 
Table 1.--Alphabetical listing of current and historical gaging stations maintained by the U.S. Geological Survey

September $90,198 \%$-Continued

[Symbols defined on last page of report]

\begin{tabular}{|c|c|c|c|c|c|c|c|}
\hline $\begin{array}{c}\text { Station } \\
\text { Number }\end{array}$ & Station & $\begin{array}{l}\text { Type } \\
\text { of } \\
\text { Data }\end{array}$ & $\begin{array}{l}\text { Q.W. } \\
\text { Para- } \\
\text { meters }\end{array}$ & $\begin{array}{l}\text { Stage } \\
\text { Source }\end{array}$ & $\begin{array}{l}\text { Coop- } \\
\text { erator }\end{array}$ & $\begin{array}{c}\text { Drainage } \\
\text { Area } \\
\left(m i l e^{2}\right)\end{array}$ & Period of Record \\
\hline $\begin{array}{l}\emptyset 7229442 \\
67171165 \\
67171165 \\
67171165 \\
67246591\end{array}$ & $\begin{array}{l}\text { E Elm Ck nr Moore } \\
\text { E Fk Big Ck nr Hollow } \\
\text { E Fk Big Ck nr Hollow } \\
\text { E Fk Big Ck nr Hollow } \\
\text { E Pond Outlet to Mule Ck nr MeCurtain }\end{array}$ & $\begin{array}{l}\text { QWP } \\
\text { QWMP } \\
\text { PR } \\
\text { SED } \\
\text { QWP }\end{array}$ & $\begin{array}{l}C P \\
C P \\
S \\
C P\end{array}$ & & & $\begin{array}{l}14.4 \\
14.4 \\
14.4\end{array}$ & $\begin{array}{l}1980 \\
1980-83 \\
1980-83 \\
1980-83 \\
1979-82\end{array}$ \\
\hline 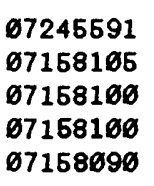 & $\begin{array}{l}\text { E Pond Outlet to Mule Ck nr McCurtain } \\
\text { Eagle Chief Ck at Cleo Springs } \\
\text { Eagle Chief Ck nr Aline } \\
\text { Eagle Chief Ck nr Aline } \\
\text { Eagle Chief Ck nr Carmen }\end{array}$ & $\begin{array}{l}\text { PR } \\
\text { QWP } \\
\text { LF } \\
\text { QWP } \\
\text { QWP }\end{array}$ & $\begin{array}{l}C P \\
C P \\
C P\end{array}$ & & & $\begin{array}{l}406 . \\
486 .\end{array}$ & $\begin{array}{l}1979-82 \\
1952-55 \\
* 1953-65,61-73 \\
1963-65,58-59 \\
1953-54\end{array}$ \\
\hline $\begin{array}{l}67299725 \\
67304167 \\
67304500 \\
67304600 \\
67304500\end{array}$ & $\begin{array}{l}\text { Eldorado Spring } n r \text { Eldorado } \\
\text { Elk Ck nr Elk City } \\
\text { Elk Ck nr Hobart } \\
\text { Elk Ck nr Hobart } \\
\text { Elk Ck nr Hobart }\end{array}$ & $\begin{array}{l}\text { QWP } \\
\text { QWP } \\
\text { STR } \\
\text { QWP } \\
\text { QWMP }\end{array}$ & $\begin{array}{l}C P \\
C P \\
C P \\
C N P\end{array}$ & OBS & $\begin{array}{l}D G \\
D G\end{array}$ & $\begin{array}{l}549 . \\
549 . \\
549\end{array}$ & $\begin{array}{l}1950 \\
1960 \\
* * 1904-68,49- \\
* * 1949,54-63,70-87 \\
1975-79\end{array}$ \\
\hline 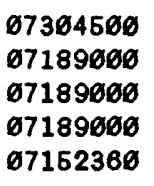 & $\begin{array}{l}\text { Elk Ck nr Hobart } \\
\text { Elk R nr Tiff City MO } \\
\text { Elk R nr Tiff City MO } \\
\text { Elk R nr Tiff City MO } \\
\text { Elm Ck nr Foraker }\end{array}$ & $\begin{array}{l}\text { QWD } \\
\text { STR } \\
\text { QWP } \\
\text { QWMP } \\
\text { CS }\end{array}$ & $\begin{array}{l}P \\
C P \\
C N P\end{array}$ & DCP & $\begin{array}{l}\text { DG } \\
\text { LGC }\end{array}$ & $\begin{array}{l}649 . \\
872 . \\
872 . \\
872 . \\
18.2\end{array}$ & $\begin{array}{l}1949-51,58-63,69-87 \\
1939- \\
1948-49,51-58,68-61 \\
1973-77 \\
1984-76\end{array}$ \\
\hline $\begin{array}{l}\emptyset 7229441 \\
67363395 \\
67303506 \\
\varnothing 7303606 \\
67363560\end{array}$ & $\begin{array}{l}\text { Elm Ck } n r \text { Moore } \\
\text { Elm Fk } N \text { Fk Red } R \text { at Salton Crossing } \\
\text { Elm Fk } N \text { Fk Red } R \text { nr Mangum } \\
\text { Elm Fk } N \text { Fk Red } R \text { nr Mangum } \\
\text { Elm Fk } N \text { Fk Red R nr Mangum }\end{array}$ & $\begin{array}{l}\text { QWP } \\
\text { QWH } \\
\text { STR } \\
\text { QWH } \\
\text { QWMP }\end{array}$ & $\begin{array}{l}C P \\
C P \\
C P \\
C N P\end{array}$ & & & $\begin{array}{l}411 . \\
838 . \\
838 . \\
838\end{array}$ & $\begin{array}{l}1960-61 \\
1980-61,73-79 \\
* 1905-68,65-76 \\
* * 1951,1888-76 \\
1976-79\end{array}$ \\
\hline $\begin{array}{l}\emptyset 7363420 \\
\varnothing 730342 \emptyset \\
\varnothing 7363466 \\
\varnothing 7303466 \\
\varnothing 736346 \varnothing\end{array}$ & $\begin{array}{l}\text { Elm Fk } N \text { Fk Red } R n r \text { Reed } \\
\text { EIm Fk } N \text { Fk Red } R n r \text { Reed } \\
\text { Elm Fk } N \text { Fk Red } R n r \text { Vinson } \\
\text { Elm Fk } N \text { Fk Red } R n r \text { Vinson } \\
\text { EIm Fk } N \text { Fork Red R nr Carl }\end{array}$ & $\begin{array}{l}\text { STR } \\
\text { QWP } \\
\text { PR } \\
\text { QWP } \\
\text { PR }\end{array}$ & $\begin{array}{l}\mathrm{CP} \\
\mathrm{CP}\end{array}$ & & & $\begin{array}{l}679 . \\
679 . \\
428 \\
428 \\
416\end{array}$ & $\begin{array}{l}1985-67 \\
1978 \\
1978-81 \\
1978-81 \\
* 1959-79,80-82\end{array}$ \\
\hline 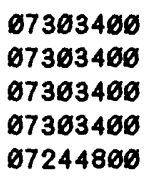 & $\begin{array}{l}\text { Elm Fk N Fork Red R nr Carl } \\
\text { Elm Fk N Fork Red R nr Carl } \\
\text { Elm Fk N Fork Red R nr Carl } \\
\text { Elm Fk N Fork Red R nr Carl } \\
\text { Eufaula Lake nr Brooken }\end{array}$ & $\begin{array}{l}\text { QWP } \\
\text { QWMP } \\
\text { SED } \\
\text { STR } \\
\text { RES }\end{array}$ & $\begin{array}{l}\text { CP } \\
\text { CNP } \\
S\end{array}$ & DCP & $\begin{array}{l}J \\
C\end{array}$ & $\begin{array}{r}416 \\
416 \\
416 \\
416 \\
47622\end{array}$ & $\begin{array}{l}1960-63,68-82 \\
1976-77 \\
1978-79 \\
1959-79 \\
1964-\end{array}$ \\
\hline $\begin{array}{l}\emptyset 72448 \varnothing \emptyset \\
\varnothing 733792 \emptyset \\
\emptyset 732825 \emptyset \\
\emptyset 73283 \varnothing \emptyset \\
\emptyset 73 \varnothing 34 \varnothing 2\end{array}$ & $\begin{array}{l}\text { Eufaula Lake nr Brooken } \\
\text { Fifteen Ck nr Glover } \\
\text { Finn Ck nr Payne } \\
\text { Finn Ck nr Story } \\
\text { Fish Ck nr Vinson }\end{array}$ & $\begin{array}{l}\text { QWD } \\
\text { CS } \\
\text { QWP } \\
\text { QWP } \\
\text { PR }\end{array}$ & $\begin{array}{l}\mathrm{CP} \\
\mathrm{CP} \\
\mathrm{CP}\end{array}$ & & & $\begin{array}{c}47522 . \\
1.23 \\
67.2 \\
31.5\end{array}$ & $\begin{array}{l}1965-67 \\
1967-73 \\
1961 \\
1951-60 \\
1978-79\end{array}$ \\
\hline
\end{tabular}

* Continuous streamflow records for this period.

** Some records in intervening years.

*** Some records may have been collected previously. 
Table 1.--Alphabetical listing of current and historical gaging stations maintained by the U.S. Geological Survey

September 30, 1987--Continued

[Symbols defined on last page of report]

\begin{tabular}{|c|c|c|c|c|c|c|c|}
\hline $\begin{array}{c}\text { Station } \\
\text { Number }\end{array}$ & Station & $\begin{array}{c}\text { Type } \\
\text { of } \\
\text { Data }\end{array}$ & $\begin{array}{l}\text { Q.W. } \\
\text { Para- } \\
\text { meters }\end{array}$ & $\begin{array}{l}\text { Stage } \\
\text { Source }\end{array}$ & $\begin{array}{l}\text { Coop- } \\
\text { erator }\end{array}$ & $\begin{array}{c}\text { Drainage } \\
\text { Area } \\
(\mathrm{mile})\end{array}$ & Period of Record \\
\hline $\begin{array}{l}\emptyset 73634 \varnothing 2 \\
672421 \varnothing 9 \\
\varnothing 715651 \emptyset \\
671777 \varnothing \emptyset \\
\varnothing 71777 \varnothing \emptyset\end{array}$ & $\begin{array}{l}\text { Fish Ck nr Vinson } \\
\text { Fish Ck nr Wetumka } \\
\text { Flagg Springs Ck Trib nr Boise City } \\
\text { Flat Rock at Hwy } 75 \text { at Tulsa } \\
\text { Flat Rock at Hwy } 76 \text { at Tulsa }\end{array}$ & $\begin{array}{l}\text { QWP } \\
\text { QWP } \\
\text { CS } \\
\text { STR } \\
\text { QWH }\end{array}$ & $\begin{array}{l}C P \\
C P\end{array}$ & & $\begin{array}{l}P \\
P\end{array}$ & $\begin{array}{l}31.5 \\
5.15\end{array}$ & $\begin{array}{l}1978-79 \\
1980 \\
1985-72 \\
1986- \\
1987-\end{array}$ \\
\hline 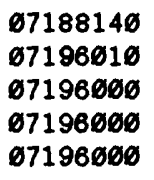 & $\begin{array}{l}\text { Flint Br nr Peoria } \\
\text { Flint Ck Trib nr Flint } \\
\text { Flint Ck nr Kansas } \\
\text { Flint Ck nr Kansas } \\
\text { Flint Ck nr Kansas }\end{array}$ & $\begin{array}{l}\text { CS } \\
\text { CS } \\
\text { QWP } \\
\text { QWMP } \\
\text { STR }\end{array}$ & $\begin{array}{l}\text { CP } \\
\text { CNP }\end{array}$ & & HG & $\begin{array}{l}4.90 \\
6.94 \\
110 . \\
110 \\
110\end{array}$ & $\begin{array}{l}1964-85 \\
1966-72 \\
1955-61,63 \\
1975-79 \\
1979-\end{array}$ \\
\hline 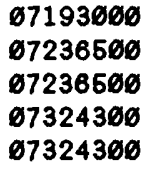 & $\begin{array}{l}\text { Fort Gibson Lake nr Fort Gibson } \\
\text { Fort Supply Lake nr Fort Supply } \\
\text { Fort Supply Lake nr Fort Supply } \\
\text { Foss Reservoir nr Foss } \\
\text { Foss Reservoir nr Foss }\end{array}$ & $\begin{array}{l}\text { RES } \\
\text { RES } \\
\text { QWP } \\
\text { RESP } \\
\text { QWP }\end{array}$ & $\begin{array}{l}C P \\
C P\end{array}$ & $\begin{array}{l}\text { DCP } \\
\text { DCP } \\
\text { RES }\end{array}$ & $\begin{array}{l}C \\
C \\
\text { JG } \\
\text { VG }\end{array}$ & $\begin{array}{r}12492 \\
1736 \\
1736 \\
1496 \\
1496\end{array}$ & $\begin{array}{l}1949- \\
1942- \\
1949,56,53 \\
1961- \\
1963-87\end{array}$ \\
\hline $\begin{array}{l}\emptyset 73243 \varnothing \emptyset \\
\varnothing 72475 \varnothing \emptyset \\
\emptyset 72475 \varnothing \emptyset \\
\emptyset 72475 \varnothing \emptyset \\
\varnothing 724745 \varnothing\end{array}$ & $\begin{array}{l}\text { Foss Reservoir nr Foss } \\
\text { Fourche Maline nr Red Oak } \\
\text { Fourche Maline nr Red Oak } \\
\text { Fourche Maline nr Red Oak } \\
\text { Fourche Maline nr Wilburton }\end{array}$ & $\begin{array}{l}\text { QWMP } \\
\text { STR } \\
\text { QWP } \\
\text { QWMP } \\
\text { STR }\end{array}$ & $\begin{array}{l}\text { CNP } \\
\text { CP } \\
\text { CNP }\end{array}$ & & JG & $\begin{array}{l}1496 \\
122 \\
122 \\
122 \\
56.2\end{array}$ & $\begin{array}{l}1976-77 \\
1938- \\
1952,54,56-66,63 \\
1978-79 \\
1978-81\end{array}$ \\
\hline $\begin{array}{l}\emptyset 724745 \emptyset \\
\emptyset 724746 \emptyset \\
\emptyset 724745 \emptyset \\
\emptyset 733652 \emptyset \\
\emptyset 73269 \emptyset \emptyset\end{array}$ & $\begin{array}{l}\text { Fourche Maline nr Wilburton } \\
\text { Fourche Maline nr Wilburton } \\
\text { Fourche Maline nr Wilburton } \\
\text { Frazier Ck nr Oleta } \\
\text { Ft Cobb Reservoir nr Ft Cobb }\end{array}$ & $\begin{array}{l}\text { QWH } \\
\text { SED } \\
\text { QWP } \\
\text { CS } \\
\text { RESP }\end{array}$ & $\begin{array}{l}P \\
S \\
C N P\end{array}$ & RES & $\begin{array}{l}H G \\
N G\end{array}$ & $\begin{array}{r}66.2 \\
56.2 \\
66.2 \\
19.4 \\
364 .\end{array}$ & $\begin{array}{l}1978-8 \emptyset \\
1978-81 \\
1978-81 \\
1964-86 \\
1959-\end{array}$ \\
\hline $\begin{array}{l}\emptyset 732590 \emptyset \\
6723205 \emptyset \\
\emptyset 7231956 \\
\emptyset 7231955 \\
\emptyset 723260 \varnothing\end{array}$ & $\begin{array}{l}\text { Ft Cobb Reservoir nr Ft Cobb } \\
\text { Gaines Ck nr Canadian } \\
\text { Gaines Ck nr Higgins } \\
\text { Gaines Ck nr Higgins } \\
\text { Gaines Ck nr Krobs }\end{array}$ & $\begin{array}{l}\text { QWP } \\
\text { QWP } \\
\text { PR } \\
\text { QWP } \\
\text { STRP }\end{array}$ & $\begin{array}{l}\mathrm{CP} \\
\mathrm{CP} \\
\mathrm{CNP}\end{array}$ & & & 304 & $\begin{array}{l}196 \emptyset-62 \\
196 \emptyset-62 \\
1978-8 \emptyset \\
1978-8 \emptyset \\
1943-63\end{array}$ \\
\hline $\begin{array}{l}\emptyset 723206 \emptyset \\
673653 \varnothing 2 \\
\varnothing 73379 \varnothing \emptyset \\
\varnothing 73379 \varnothing \emptyset \\
\varnothing 733796 \varnothing\end{array}$ & $\begin{array}{l}\text { Gaines Ck nr Krebs } \\
\text { Glenn Ck nr Cold Spring } \\
\text { Glover Ck nr Glover } \\
\text { Glover Ck nr Glover } \\
\text { Glover Ck nr Glover }\end{array}$ & $\begin{array}{l}\text { QWP } \\
\text { QWP } \\
\text { STRP } \\
\text { QWP } \\
\text { QWMP }\end{array}$ & $\begin{array}{l}\mathrm{CP} \\
\mathrm{CP} \\
\mathrm{CP} \\
\mathrm{CNP}\end{array}$ & DAR & C & $\begin{array}{l}315 . \\
315 . \\
315 .\end{array}$ & $\begin{array}{l}1946-47,50-51,60-62 \\
1961 \\
1981- \\
1949,53,62-63 \\
1975-79\end{array}$ \\
\hline $\begin{array}{l}\emptyset 723259 \emptyset \\
6723258 \emptyset \\
\varnothing 7162290 \\
\emptyset 71500 \varnothing \emptyset \\
\varnothing 716000 \varnothing\end{array}$ & $\begin{array}{l}\text { Goff Ck } n r \text { Guymon } \\
\text { Goff Ck } n r \text { Hough } \\
\text { Greasy Ck nr Watchorn } \\
\text { Great Salt Plains Lake } n r \text { Jet } \\
\text { Great Salt Plains Lake } n r \text { Jet }\end{array}$ & $\begin{array}{l}\text { LF } \\
\text { LF } \\
\text { STR } \\
\text { RES } \\
\text { QWP }\end{array}$ & $C P$ & $\begin{array}{l}\text { RES } \\
\text { DCP }\end{array}$ & C & $\begin{array}{l}510 . \\
470 \\
28.0 \\
3200 \\
3200\end{array}$ & $\begin{array}{l}1964-68 \\
1968-71 \\
1974-76 \\
1941- \\
1950\end{array}$ \\
\hline
\end{tabular}

Continuous streamflow records for this period.

Some records in intervening years.

Some records may have been collected previously. 
Table 1.--Alphabetical listing of current and historical gaging stations maintained by the U.S. Geological Survey

September 30, 1987--Continued

[Symbols defined on last page of report]

\begin{tabular}{|c|c|c|c|c|c|c|c|}
\hline $\begin{array}{r}\text { Station } \\
\text { Number }\end{array}$ & Station Name & $\begin{array}{l}\text { Type } \\
\text { of } \\
\text { Data }\end{array}$ & $\begin{array}{l}\text { Q.W. } \\
\text { Para- } \\
\text { metors }\end{array}$ & $\begin{array}{l}\text { Stage } \\
\text { Source }\end{array}$ & $\begin{array}{l}\text { Coop- } \\
\text { orator }\end{array}$ & $\begin{array}{c}\text { Drainage } \\
\text { Area } \\
(m i l e)\end{array}$ & Period of Record \\
\hline $\begin{array}{l}\emptyset 714996 \emptyset \\
67194546 \\
\emptyset 719454 \emptyset \\
\emptyset 714836 \emptyset \\
\emptyset 7242090\end{array}$ & $\begin{array}{l}\text { Great Salt Plains NADP Site nr Jet } \\
\text { Greenleaf Ck nr Braggs } \\
\text { Greenleaf Lake nr Braggs } \\
\text { Greenwood Ck nr Winchester } \\
\text { Grief Ck nr Wetumka }\end{array}$ & $\begin{array}{l}\text { QWP } \\
\text { QWP } \\
\text { QWP } \\
\text { LF } \\
\text { QWP }\end{array}$ & $\begin{array}{l}C P \\
C P \\
C P \\
C P\end{array}$ & & JG & 41.2 & $\begin{array}{l}1984- \\
1953-66 \\
1949,51,52 \\
1972- \\
1962-63\end{array}$ \\
\hline $\begin{array}{l}\emptyset 729977 \varnothing \\
\varnothing 729978 \emptyset \\
6729978 \emptyset \\
6729978 \emptyset \\
6729978 \emptyset\end{array}$ & $\begin{array}{l}\text { Gypsum Ck } n r \text { Creta } \\
\text { Gypsum Ck } n r \text { Ol ustee } \\
\text { Gypsum Ck } n r \text { Ol usteo } \\
\text { Gypsum Ck } n r \text { Olusteo } \\
\text { Gypsum Ck } n r \text { Ol ustee }\end{array}$ & $\begin{array}{l}\text { QWP } \\
\text { LF } \\
\text { QWP } \\
\text { MISC } \\
\text { STR }\end{array}$ & $\begin{array}{l}C P \\
C P\end{array}$ & & $J$ & $\begin{array}{l}167 . \\
167 . \\
167 . \\
167 .\end{array}$ & $\begin{array}{l}1961 \\
1954-67,65-73 \\
1956-57 \\
1987- \\
1987-\end{array}$ \\
\hline $\begin{array}{l}\emptyset 7165060 \\
\varnothing 716506 \emptyset \\
\emptyset 7316 \varnothing 7 \emptyset \\
\emptyset 72298 \varnothing 1 \\
6717472 \emptyset\end{array}$ & $\begin{array}{l}\text { Heyburn Lake } n r \text { Heyburn } \\
\text { Heyburn Lake nr Heyburn } \\
\text { Hickory Ck nr Marietta } \\
\text { Hog Ck nr Stella } \\
\text { Hogshooter Ck Trib nr Bartlesville }\end{array}$ & $\begin{array}{l}\text { RES } \\
\text { QWP } \\
\text { LF } \\
\text { QWP } \\
\text { CS }\end{array}$ & $\begin{array}{l}\mathrm{CP} \\
\mathrm{CP}\end{array}$ & $D C P$ & HG & $\begin{array}{l}123 . \\
123 \\
116 \\
6.94\end{array}$ & $\begin{array}{l}1950- \\
1961 \\
1964-73 \\
1960-61 \\
1965-86\end{array}$ \\
\hline $\begin{array}{l}\emptyset 7249422 \\
\emptyset 7249422 \\
\emptyset 7249422 \\
\emptyset 7177410 \\
07176950\end{array}$ & $\begin{array}{l}\text { Holi-Tuska Ck nr Panama } \\
\text { Holi-Tuska Ck nr Panama } \\
\text { Holi-Tuska Ck nr Panama } \\
\text { Hominy Ck blw Skiatook Lk nr Skiatook } \\
\text { Hominy Ck nr Hominy }\end{array}$ & $\begin{array}{l}\text { STRP } \\
\text { QWP } \\
\text { SED } \\
\text { STR } \\
\text { QWP }\end{array}$ & $\begin{array}{l}S \\
C P\end{array}$ & & C & $\begin{array}{r}4.39 \\
4.39 \\
4.39 \\
364 .\end{array}$ & $\begin{array}{l}1978-81 \\
1978-81 \\
1978-81 \\
1984- \\
1950-53,66,65-66\end{array}$ \\
\hline $\begin{array}{l}07177000 \\
67177000 \\
6717746 \emptyset \\
\emptyset 7329870 \\
0732987 \emptyset\end{array}$ & $\begin{array}{l}\text { Hominy Ck nr Skiatook } \\
\text { Hominy Ck nr Skiatook } \\
\text { Hominy Ck nr Sperry } \\
\text { Honey Ck nr Davis } \\
\text { Honey Ck nr Davis }\end{array}$ & $\begin{array}{l}\text { STRP } \\
\text { QWP } \\
\text { QWP } \\
\text { CS } \\
\text { QWP }\end{array}$ & $\begin{array}{l}\mathrm{CP} \\
\mathrm{CP} \\
\mathrm{CP}\end{array}$ & & $H G$ & $\begin{array}{l}340 . \\
340 . \\
18.7 \\
18.7\end{array}$ & $\begin{array}{l}1944-80 \\
1948-53,65-66 \\
1952-53 \\
1964-85 \\
1953,55-56\end{array}$ \\
\hline $\begin{array}{l}07328860 \\
67189720 \\
67189700 \\
07306000 \\
67163900\end{array}$ & $\begin{array}{l}\text { Honey Ck nr Turner Falls } \\
\text { Horse Ck Trib nr Afton } \\
\text { Horse Ck at Afton } \\
\text { Horse Ck nr Mountain Park } \\
\text { House Ck nr Terlton }\end{array}$ & $\begin{array}{l}\text { QWP } \\
\text { CS } \\
\text { CS } \\
\text { STR } \\
\text { QWP }\end{array}$ & $C P$ & & HG & $\begin{array}{l}.81 \\
21.9 \\
11.1 \\
0.81\end{array}$ & $\begin{array}{l}1949-51,54 \\
1966-72 \\
1966-85 \\
1966 \\
1953-55\end{array}$ \\
\hline $\begin{array}{l}07168140 \\
07336600 \\
\emptyset 71726 \varnothing \emptyset \\
\emptyset 7196380 \\
67197520\end{array}$ & $\begin{array}{l}\text { Hoyle Ck nr Ames } \\
\text { Hugo Lake nr Hugo } \\
\text { Hulah Lake nr Hulah } \\
\text { Illinois R Trib nr Tahlequah } \\
\text { Illinois R at Tenkiller L TW }\end{array}$ & $\begin{array}{l}\text { QWP } \\
\text { RESP } \\
\text { RES } \\
\text { CS } \\
\text { STGU }\end{array}$ & $\mathrm{CP}$ & $D C P$ & $\begin{array}{l}\mathrm{C} \\
\mathrm{C} \\
\mathrm{C}\end{array}$ & $\begin{array}{l}1769 . \\
732 . \\
3.69\end{array}$ & $\begin{array}{l}1962-54 \\
1974- \\
1950- \\
1985-75 \\
1986-\end{array}$ \\
\hline 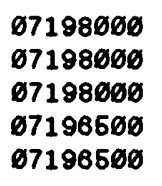 & $\begin{array}{l}\text { Illinois } R \mathrm{nr} \text { Gore } \\
\text { Illinois } \mathrm{nr} \text { Gore } \\
\text { Illinois } R \mathrm{nr} \text { Gore } \\
\text { Illinois } \mathrm{nr} \text { Tahlequah } \\
\text { Illinois } \mathrm{nr} \text { Tahlequah }\end{array}$ & $\begin{array}{l}\text { STR } \\
\text { QWP } \\
\text { QWMP } \\
\text { STR } \\
\text { QWP }\end{array}$ & $\begin{array}{l}C P \\
C N P \\
C P\end{array}$ & $\begin{array}{l}D C P \\
D C P\end{array}$ & $\begin{array}{l}C \\
J G \\
J G\end{array}$ & $\begin{array}{r}1626 . \\
1626 . \\
1626 . \\
959 . \\
959 .\end{array}$ & $\begin{array}{l}1939 * * *, 48,52,54- \\
1948- \\
1973-79 \\
1935- \\
1980-61\end{array}$ \\
\hline
\end{tabular}

* Continuous streamflow records for this poriod.

** Some records in intervening years.

*** Some records may have been collected previously. 
Table 1.--Alphabetical listing of current and historical gaging stations maintained by the U.S. Geological Survey

September so, 1987--Continued

[Symbols defined on last page of report]

\begin{tabular}{|c|c|c|c|c|c|c|c|}
\hline $\begin{array}{r}\text { Station } \\
\text { Number }\end{array}$ & Station Name & $\begin{array}{l}\text { Type } \\
\text { of } \\
\text { Data }\end{array}$ & $\begin{array}{l}\text { Q.W. } \\
\text { Para- } \\
\text { meters }\end{array}$ & $\begin{array}{l}\text { Stage } \\
\text { Source }\end{array}$ & $\begin{array}{l}\text { Coop- } \\
\text { erator }\end{array}$ & $\begin{array}{c}\text { Drainage } \\
\text { Area } 2 \\
\left(m i l \theta^{2}\right)\end{array}$ & Period of Record \\
\hline 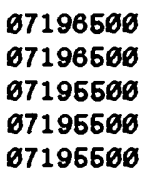 & $\begin{array}{l}\text { Illinois } R n r \text { Tahlequah } \\
\text { Illinois } R n r \text { Tahlequah } \\
\text { Illinois } R \text { nr Watts } \\
\text { Illinois } R \text { nr Watts } \\
\text { Illinois } R \text { nr Watts }\end{array}$ & $\begin{array}{l}\text { QWMP } \\
\text { SED } \\
\text { STR } \\
\text { QWP } \\
\text { QWMP }\end{array}$ & $\begin{array}{l}\text { CNP } \\
\mathbf{S} \\
\text { CNP } \\
\text { CNP }\end{array}$ & $D C P$ & $\begin{array}{l}\mathrm{C} \\
\mathrm{C}\end{array}$ & $\begin{array}{l}959 . \\
959 . \\
636 \\
636 \\
636\end{array}$ & $\begin{array}{l}1976-79 \\
1976- \\
1965- \\
1966-61,63,69-73 \\
1976-79\end{array}$ \\
\hline $\begin{array}{l}\varnothing 7301496 \\
\varnothing 7237696 \\
\varnothing 733225 \varnothing \\
\varnothing 7336786 \\
\varnothing 724684 \varnothing\end{array}$ & $\begin{array}{l}\text { Indian Ck nr Carter } \\
\text { Indian Ck nr Woodward } \\
\text { Island Bayou nr Albany } \\
\text { Jacks Fk Ck at Sardis Lake } \\
\text { Jackson Ck nr Stigler }\end{array}$ & $\begin{array}{l}\text { CS } \\
\text { QWP } \\
\text { LF } \\
\text { STRP } \\
\text { PR }\end{array}$ & $\mathrm{CP}$ & & $C$ & $\begin{array}{l}24.9 \\
132 . \\
276 . \\
7.33\end{array}$ & $\begin{array}{l}1966-76 \\
1961-67 \\
1966-73 \\
1984- \\
1980-81\end{array}$ \\
\hline $\begin{array}{l}\varnothing 7246 \varnothing 4 \varnothing \\
\varnothing 7245 \varnothing 4 \varnothing \\
\varnothing 72494 \varnothing \emptyset \\
\varnothing 72494 \varnothing \varnothing \\
\varnothing 72494 \varnothing \varnothing\end{array}$ & $\begin{array}{l}\text { Jackson Ck nr Stigler } \\
\text { Jackson Ck } n r \text { Stigler } \\
\text { James Fk } n r \text { Hackett AR } \\
\text { James Fk } n r \text { Hackett AR } \\
\text { James Fk } n r \text { Hackett AR }\end{array}$ & $\begin{array}{l}\text { QWP } \\
\text { SED } \\
\text { STR } \\
\text { QWP } \\
\text { SED }\end{array}$ & $\begin{array}{l}\text { CNP } \\
S \\
\text { CNP } \\
S\end{array}$ & & $W$ & $\begin{array}{l}7.33 \\
7.33 \\
148 . \\
148 . \\
148 .\end{array}$ & $\begin{array}{l}1980-81 \\
1980-81 \\
1968- \\
1960-61,76-81 \\
1976-81\end{array}$ \\
\hline $\begin{array}{l}\varnothing 724941 \emptyset \\
\varnothing 724941 \emptyset \\
\varnothing 724941 \varnothing \\
\varnothing 722942 \varnothing \\
\varnothing 714813 \varnothing\end{array}$ & $\begin{array}{l}\text { James Fk nr Williams } \\
\text { James Fk nr Williams } \\
\text { James Fk nr Williams } \\
\text { Julian Ck Trib nr Asher } \\
\text { Kaw Lake nr Ponca City }\end{array}$ & $\begin{array}{l}\text { PR } \\
\text { QWP } \\
\text { SED } \\
\text { CS } \\
\text { RES }\end{array}$ & $\begin{array}{l}\text { CNP } \\
S\end{array}$ & $D C P$ & $\begin{array}{l}H G \\
C\end{array}$ & $\begin{array}{l}198 . \\
198 . \\
198 . \\
2.28 \\
46630\end{array}$ & $\begin{array}{l}1976-81 \\
1976-81 \\
1976-81 \\
1964-86 \\
1977-\end{array}$ \\
\hline $\begin{array}{l}\emptyset 716316 \emptyset \\
\varnothing 716420 \emptyset \\
\varnothing 71642 \varnothing \emptyset \\
\varnothing 71642 \varnothing \emptyset \\
\varnothing 733576 \varnothing\end{array}$ & $\begin{array}{l}\text { Keystone Lake nr Cleveland } \\
\text { Keystone Lake nr Sand Springs } \\
\text { Keystone Lake nr Sand Springs } \\
\text { Keystone Lake nr Sand Springs } \\
\text { Kiamichi R Trib nr Albion }\end{array}$ & $\begin{array}{l}\text { QWP } \\
\text { RES } \\
\text { QWP } \\
\text { QWMP } \\
\text { CS }\end{array}$ & $\begin{array}{l}\mathrm{CP} \\
\mathrm{CP} \\
\mathrm{COP}\end{array}$ & DCP & C & $\begin{array}{c}74606 . \\
74606 \\
74606 . \\
1.50\end{array}$ & $\begin{array}{l}1966-74 \\
1964- \\
1965-76 \\
1973-75 \\
1965-72\end{array}$ \\
\hline $\begin{array}{l}67336260 \\
67336200 \\
67336500 \\
67336500 \\
67336700\end{array}$ & $\begin{array}{l}\text { Kiamichi R nr Antlers } \\
\text { Kiamichi R nr Antlers } \\
\text { Kiamichi R } n r \text { Belzoni } \\
\text { Kiamichi R nr Belzoni } \\
\text { Kiamichi R nr Big Cedar }\end{array}$ & $\begin{array}{l}\text { STRP } \\
\text { QWMP } \\
\text { STRP } \\
\text { QWD } \\
\text { STR }\end{array}$ & $\begin{array}{l}\text { CNP } \\
C P\end{array}$ & DAR & c & $\begin{array}{l}1138 \\
1138 \\
1423 \\
1423 \\
46.1\end{array}$ & $\begin{array}{l}1972- \\
1976-79 \\
1926-72 \\
1948-64,62-63 \\
1966-\end{array}$ \\
\hline $\begin{array}{l}\varnothing 7336700 \\
\varnothing 73367 \varnothing 0 \\
\varnothing 7336700 \\
\varnothing 73367 \varnothing 0 \\
\varnothing 7336790\end{array}$ & $\begin{array}{l}\text { Kiamichi R nr Big Codar } \\
\text { Kiamichi R } n r \text { Big Codar } \\
\text { Kiamichi R nr Big Codar } \\
\text { Kiamichi R nr Big Codar } \\
\text { Kiamichi R nr Clayton }\end{array}$ & $\begin{array}{l}\text { QWP } \\
\text { QWMP } \\
\text { SED } \\
\text { QWP } \\
\text { QWMP }\end{array}$ & $\begin{array}{l}\text { BCNPR } \\
\text { CNP } \\
\text { S } \\
\text { BCNPRS } \\
\text { CNP }\end{array}$ & & $\begin{array}{l}A \\
C A \\
A\end{array}$ & $\begin{array}{l}40.1 \\
40.1 \\
40.1 \\
40.1 \\
768 .\end{array}$ & $\begin{array}{l}1966- \\
1973-79 \\
1974- \\
1982- \\
1977\end{array}$ \\
\hline $\begin{array}{l}\emptyset 7336790 \\
67336700 \\
\varnothing 7336700 \\
\varnothing 7169200 \\
67159200\end{array}$ & $\begin{array}{l}\text { Kiamichi R nr Clayton } \\
\text { Kiamichi R nr Sawyer } \\
\text { Kiamichi R nr Sawyer } \\
\text { Kingfisher Ck nr Kingfisher } \\
\text { Kingfisher Ck nr Kingfisher }\end{array}$ & $\begin{array}{l}\text { STRP } \\
\text { QWP } \\
\text { QWMP } \\
\text { CS } \\
\text { QWP }\end{array}$ & $\begin{array}{l}C P \\
C N P \\
C P\end{array}$ & & JG & $\begin{array}{l}167 . \\
167 .\end{array}$ & $\begin{array}{l}1980- \\
1962 \\
1978-79 \\
* 1966-76,71-83 \\
1969\end{array}$ \\
\hline
\end{tabular}

* Continuous streamflow records for this poriod.

** Some records in intervening years.

*** Some records may have been collected previously. 
Table 1.--Alphabetical listing of current and historical gaging stations maintained by the U.S. Geological Survey

September 30, 1987--Cont inued

[Symbols defined on last page of report]

\begin{tabular}{|c|c|c|c|c|c|c|c|}
\hline $\begin{array}{l}\text { Station } \\
\text { Number }\end{array}$ & Station & $\begin{array}{l}\text { Type } \\
\text { of } \\
\text { Data }\end{array}$ & $\begin{array}{l}\text { Q.W. } \\
\text { Para- } \\
\text { meters }\end{array}$ & $\begin{array}{l}\text { Stage } \\
\text { Source }\end{array}$ & $\begin{array}{l}\text { Coop- } \\
\text { erator }\end{array}$ & $\begin{array}{c}\text { Drainage } \\
\text { Area } \\
(m i l e)\end{array}$ & Period of Record \\
\hline $\begin{array}{l}\varnothing 723425 \emptyset \\
\varnothing 72342 \emptyset \emptyset \\
\varnothing 72342 \varnothing \emptyset \\
\varnothing 73 \varnothing 26 \varnothing \emptyset \\
\varnothing 73625 \varnothing \emptyset\end{array}$ & $\begin{array}{l}\text { Kiowa Ck Trib nr Laverne } \\
\text { Kiowa Ck nr Slapout } \\
\text { Kiowa Ck nr Slapout } \\
\text { Lake Altus at Lugert } \\
\text { Lake Altus at Lugert }\end{array}$ & $\begin{array}{l}\text { CS } \\
\text { LF } \\
\text { QWP } \\
\text { RES } \\
\text { QWP }\end{array}$ & $\begin{array}{l}\mathrm{CP} \\
\mathrm{CP}\end{array}$ & RES & TQG & $\begin{array}{l}2.14 \\
371 \\
371 \\
2516 \\
2516\end{array}$ & $\begin{array}{l}1964-72 \\
1945,49-61,63-73 \\
1952-58,6 \emptyset \\
1943- \\
1949,56-52,55-57\end{array}$ \\
\hline $\begin{array}{l}\emptyset 71616 \varnothing \emptyset \\
\emptyset 732585 \emptyset \\
\emptyset 73 ø 899 \emptyset \\
\emptyset 724 \varnothing \varnothing \varnothing \emptyset \\
\emptyset 71914 \varnothing \emptyset\end{array}$ & $\begin{array}{l}\text { Lake Carl Blackwell nr Still water } \\
\text { Lake Ck nr Eakly } \\
\text { Lake Ellsworth nr Elgin } \\
\text { Lake Hefner Canal nr OKC } \\
\text { Lake Hudson nr Locust Grove }\end{array}$ & $\begin{array}{l}\text { QWP } \\
\text { STR } \\
\text { RESU } \\
\text { STR } \\
\text { RESP }\end{array}$ & $C P$ & RES & $\begin{array}{l}\text { UG } \\
K G \\
L G\end{array}$ & $\begin{array}{r}62 . \varnothing \\
249 . \\
11634\end{array}$ & $\begin{array}{l}1947,49,51,56 \\
1969-78 \\
1964- \\
1944- \\
1964-\end{array}$ \\
\hline $\begin{array}{l}\emptyset 73 \varnothing 96 \varnothing \emptyset \\
\varnothing 73161 \varnothing \emptyset \\
\varnothing 7196 \varnothing \varnothing \emptyset \\
\varnothing 7196 \varnothing \varnothing \emptyset \\
\varnothing 724 \varnothing 6 \varnothing \emptyset\end{array}$ & $\begin{array}{l}\text { Lake Lawtonka nr Lawton } \\
\text { Lake Murray nr Ardmore } \\
\text { Lake O', The Cherokees at Langley } \\
\text { Lake O' The Cherokees at Langley } \\
\text { Lake Overholser nr OKC }\end{array}$ & $\begin{array}{l}\text { RESU } \\
\text { QWP } \\
\text { RES } \\
\text { QWP } \\
\text { RESP }\end{array}$ & $\begin{array}{l}\mathrm{CP} \\
\mathrm{CP}\end{array}$ & $\begin{array}{l}\text { RES } \\
\text { RES } \\
\text { RES }\end{array}$ & $\begin{array}{l}\text { UG } \\
\text { LG } \\
\text { KG }\end{array}$ & $\begin{array}{l}93 . \emptyset \\
16298 . \\
16298 . \\
13221\end{array}$ & $\begin{array}{l}1966- \\
1949-63,56-57 \\
1946- \\
1948-49 \\
1937-\end{array}$ \\
\hline $\begin{array}{l}\emptyset 72416 \varnothing \emptyset \\
\varnothing 73316 \varnothing \emptyset \\
\varnothing 73316 \varnothing \emptyset \\
\varnothing 72299 \varnothing \emptyset \\
\varnothing 72299 \varnothing \emptyset\end{array}$ & $\begin{array}{l}\text { Lake Shawnee nr Shawnee } \\
\text { Lake Texoma nr Denison TX } \\
\text { Lake Texoma nr Denison TX } \\
\text { Lake Thunderbird nr Norman } \\
\text { Lake Thunderbird nr Norman }\end{array}$ & $\begin{array}{l}\text { RESU } \\
\text { RESP } \\
\text { QWP } \\
\text { RESP } \\
\text { QWP }\end{array}$ & $\begin{array}{l}\mathrm{CP} \\
\mathrm{CP}\end{array}$ & $\begin{array}{l}\text { RES } \\
\text { RES }\end{array}$ & $\begin{array}{l}C \\
\text { GM } \\
\text { JGB }\end{array}$ & $\begin{array}{l}34.0 \\
39719 . \\
39719 \\
256 \\
256\end{array}$ & $\begin{array}{l}1957-78 \\
1942- \\
1949-61 \\
1965- \\
1965-\end{array}$ \\
\hline $\begin{array}{l}\emptyset 732988 \emptyset \\
\varnothing 732988 \emptyset \\
\emptyset 723132 \emptyset \\
\varnothing 733442 \emptyset \\
\varnothing 733442 \varnothing\end{array}$ & $\begin{array}{l}\text { Lawrence Spring nr Drake } \\
\text { Lawrence Spring nr Drake } \\
\text { Leader Ck Trib nr Atwood } \\
\text { Leader Ck t Tupelo } \\
\text { Leader Ck at Tupelo }\end{array}$ & $\begin{array}{l}\text { QWP } \\
\text { QWP } \\
\text { CS } \\
\text { LF } \\
\text { QWP }\end{array}$ & $\begin{array}{l}C P \\
C P\end{array}$ & & HG & $\begin{array}{l}0.72 \\
64.3 \\
64.3\end{array}$ & $\begin{array}{l}1953 \\
1962,56-56 \\
1964-85 \\
1958-73 \\
1958,60\end{array}$ \\
\hline $\begin{array}{l}\emptyset 72498 \emptyset \emptyset \\
\varnothing 72498 \emptyset \emptyset \\
\varnothing 724123 \emptyset \\
\varnothing 717124 \emptyset \\
\varnothing 7313 \varnothing \varnothing \emptyset\end{array}$ & $\begin{array}{l}\text { Lee Ck nr Short } \\
\text { Lee Ck nr Short } \\
\text { Lightning Ck at Sw74 OKC } \\
\text { Lightning Ck nr Alluwe } \\
\text { Little Beaver Ck nr Duncan }\end{array}$ & $\begin{array}{l}\text { QWP } \\
\text { QWMP } \\
\text { CS } \\
\text { QWP } \\
\text { STR }\end{array}$ & $\begin{array}{l}\mathrm{CP} \\
\mathrm{CNP} \\
\mathrm{CP}\end{array}$ & & & $\begin{array}{l}236 . \\
236 . \\
3.96 \\
158 .\end{array}$ & $\begin{array}{l}1958-61 \\
1976-77 \\
1976-81 \\
1962-63,69 \\
1949-63\end{array}$ \\
\hline $\begin{array}{l}\emptyset 7313 \varnothing \varnothing \emptyset \\
\varnothing 7313 \varnothing \varnothing 9 \\
\varnothing 731296 \emptyset \\
\varnothing 719685 \emptyset \\
\varnothing 717431 \emptyset\end{array}$ & $\begin{array}{l}\text { Little Beaver Ck nr Duncan } \\
\text { Little Beaver Ck nr Gas City } \\
\text { Little Beaver Ck nr Marlow } \\
\text { Little Cabin Ck nr Vinita } \\
\text { Little Caney R blw Copan Lake nr Copan }\end{array}$ & $\begin{array}{l}\text { QWP } \\
\text { QWP } \\
\text { CS } \\
\text { QWP } \\
\text { STR }\end{array}$ & $\begin{array}{l}C P \\
C P \\
C P\end{array}$ & & C & $\begin{array}{l}168 . \\
35.40\end{array}$ & $\begin{array}{l}* 1948-49,58-63 \\
1981 \\
1984-75 \\
1948-51 \\
1984-\end{array}$ \\
\hline $\begin{array}{l}\emptyset 71742 \varnothing \emptyset \\
\varnothing 724346 \emptyset \\
\varnothing 7328 \emptyset 4 \emptyset \\
\varnothing 72499 \varnothing \emptyset \\
\varnothing 731 \varnothing \varnothing \varnothing \emptyset\end{array}$ & $\begin{array}{l}\text { Little Caney R blw Cotton Ck nr Copan } \\
\text { Little Deep Fk Ck nr Edna } \\
\text { Little Dry Ck nr Alex } \\
\text { Little Lee Ck nr Short } \\
\text { Little Medicine Bluff Ck nr Lawton }\end{array}$ & $\begin{array}{l}\text { STRP } \\
\text { QWP } \\
\text { CS } \\
\text { QWMP } \\
\text { STR }\end{array}$ & $\begin{array}{l}\mathrm{CP} \\
\mathrm{CNP}\end{array}$ & & & $\begin{array}{l}602 . \\
6.88 \\
163 . \\
7.06\end{array}$ & $\begin{array}{l}1958-80 \\
1951-67,60-62 \\
1961-74 \\
1978-79 \\
1913-19\end{array}$ \\
\hline
\end{tabular}

* Continuous streamflow records for this period.

** Some records in intervening years.

*** Some records may have been collected previously. 
Table 1.--Alphabetical listing of current and historical gaging stations maintained by the U.S. Geological Survey

September 30, 1987--Continued

[Symbols defined on last page of report]

\begin{tabular}{|c|c|c|c|c|c|c|c|}
\hline $\begin{array}{l}\text { Station } \\
\text { Number }\end{array}$ & Station & $\begin{array}{c}\text { Type } \\
\text { of } \\
\text { Data }\end{array}$ & $\begin{array}{l}\text { Q.W. } \\
\text { Para- } \\
\text { meters }\end{array}$ & $\begin{array}{l}\text { Stage } \\
\text { Source }\end{array}$ & $\begin{array}{l}\text { Coop- } \\
\text { erator }\end{array}$ & $\begin{array}{c}\text { Drainage } \\
\text { Area } \\
(\mathrm{mile})\end{array}$ & Period of Record \\
\hline 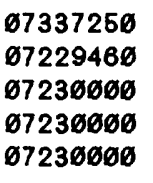 & $\begin{array}{l}\text { Little R ab Pine Ck Lake nr Wright Cty } \\
\text { Little R abv Lake Thunderbird nr Norman } \\
\text { Little R blw Lake Thunderbird nr Norman } \\
\text { Little R blw Lake Thunderbird nr Norman } \\
\text { Little R blw Lake Thunderbird nr Norman }\end{array}$ & $\begin{array}{l}\text { QWMP } \\
\text { QWP } \\
\text { STR } \\
\text { QWP } \\
\text { QWMP }\end{array}$ & $\begin{array}{l}\text { CNP } \\
\text { BCNP } \\
\text { CP } \\
\text { CNP }\end{array}$ & & $\begin{array}{l}B \\
G M\end{array}$ & $\begin{array}{l}257 \\
257 \\
257\end{array}$ & $\begin{array}{l}1976-77 \\
1983-85 \\
1952- \\
1953-65 \\
1975-79\end{array}$ \\
\hline $\begin{array}{l}\varnothing 73385 \varnothing \emptyset \\
673385 \varnothing \emptyset \\
\varnothing 73385 \varnothing \emptyset \\
\varnothing 733715 \emptyset \\
\varnothing 723 \varnothing 597\end{array}$ & $\begin{array}{l}\text { Little R blw Lukfata Ck nr Idabel } \\
\text { Little R blw Lukfata Ck } n r \text { Idabel } \\
\text { Little R blw Lukfata Ck nr Idabel } \\
\text { Little R nr Alikchi } \\
\text { Little R nr Bowlegs }\end{array}$ & $\begin{array}{l}\text { STRP } \\
\text { QWP } \\
\text { QWMP } \\
\text { QWP } \\
\text { STR }\end{array}$ & $\begin{array}{l}\text { COP } \\
\text { CNP } \\
\text { CP }\end{array}$ & $\begin{array}{l}\text { DAR } \\
\text { OBS }\end{array}$ & $c$ & $\begin{array}{l}1226 . \\
1226 . \\
1226 . \\
556 .\end{array}$ & $\begin{array}{l}1946- \\
1948-54,61-63,69-73 \\
* 1947-54,76-79 \\
1953 \\
1983-\end{array}$ \\
\hline $\begin{array}{l}67236597 \\
67236597 \\
6733916 \varnothing \\
6733716 \varnothing \\
\varnothing 733795 \varnothing\end{array}$ & $\begin{array}{l}\text { Little R nr Bowlegs } \\
\text { Little R nr Bowlegs } \\
\text { Little R nr Cerrogordo } \\
\text { Little R nr Cloudy } \\
\text { Little R nr Garvin }\end{array}$ & $\begin{array}{l}\text { QWP } \\
\text { SED } \\
\text { QWP } \\
\text { QWMP } \\
\text { QWP }\end{array}$ & $\begin{array}{l}\text { CNP } \\
S \\
C P \\
\text { CNP } \\
C P\end{array}$ & & $\begin{array}{l}B \\
B\end{array}$ & $\begin{array}{l}560 . \\
550 . \\
324 .\end{array}$ & $\begin{array}{l}1953,58,61,83- \\
1983- \\
1961-63 \\
1976-79 \\
1953\end{array}$ \\
\hline 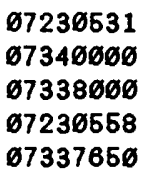 & $\begin{array}{l}\text { Little R nr Harjo } \\
\text { Little R nr Horatio AR } \\
\text { Little R nr Idabel } \\
\text { Little R nr Maud } \\
\text { Little R nr Millerton }\end{array}$ & $\begin{array}{l}\text { QWP } \\
\text { QWMP } \\
\text { STRP } \\
\text { QWP } \\
\text { QWP }\end{array}$ & $\begin{array}{l}\text { CP } \\
\text { CNP } \\
\text { CP } \\
\text { CPS }\end{array}$ & & & $\begin{array}{l}2874 . \\
1173 .\end{array}$ & $\begin{array}{l}1961 \\
1978-79 \\
1930-46 \\
1959,61 \\
1953\end{array}$ \\
\hline $\begin{array}{l}\emptyset 72295 \varnothing \emptyset \\
\varnothing 72295 \varnothing \emptyset \\
\varnothing 73372 \varnothing \varnothing \\
\varnothing 7231 \varnothing \varnothing \emptyset \\
\varnothing 7231 \varnothing \varnothing \varnothing\end{array}$ & $\begin{array}{l}\text { Little R nr Norman } \\
\text { Little R nr Norman } \\
\text { Little R } n r \text { Ringold } \\
\text { Little R } n r \text { Sasakwa } \\
\text { Little R nr Sasakwa }\end{array}$ & $\begin{array}{l}\text { STR } \\
\text { QWP } \\
\text { QWP } \\
\text { STR } \\
\text { QWP }\end{array}$ & $\begin{array}{l}C P \\
C P \\
C P\end{array}$ & & $\begin{array}{l}\text { JG } \\
\text { JG }\end{array}$ & $\begin{array}{l}120 . \\
120 . \\
885 \\
865\end{array}$ & $\begin{array}{l}1952-55 \\
1951-57,69,61 \\
1962 \\
1942- \\
1951-\end{array}$ \\
\hline 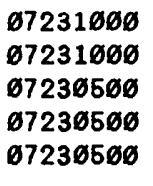 & $\begin{array}{l}\text { Little R } n r \text { Sasakwa } \\
\text { Little R } n r \text { Sasakwa } \\
\text { Little R } n r \text { Tecumseh } \\
\text { Little R } n r \text { Tecumseh } \\
\text { Little R nr Tecumseh }\end{array}$ & $\begin{array}{l}\text { QWMP } \\
\text { SED } \\
\text { STR } \\
\text { QWP } \\
\text { SED }\end{array}$ & $\begin{array}{l}\mathrm{CN} \\
\mathrm{S} \\
\mathrm{CP} \\
\mathrm{S}\end{array}$ & & C & $\begin{array}{l}865 . \\
865 \\
456 \\
466 \\
456\end{array}$ & $\begin{array}{l}1975-79 \\
1976 \\
1943- \\
1951-63 \\
* * * 1973-78\end{array}$ \\
\hline $\begin{array}{l}\emptyset 73375 \varnothing \emptyset \\
673376 \varnothing \emptyset \\
673375 \varnothing \emptyset \\
6722828 \emptyset \\
67245119\end{array}$ & $\begin{array}{l}\text { Little R nr Wright City } \\
\text { Little R nr Wright City } \\
\text { Little R nr Wright City } \\
\text { Little Robe Ck nr Oakwood } \\
\text { Little Vian Ck nr Vian }\end{array}$ & $\begin{array}{l}\text { STRP } \\
\text { QWP } \\
\text { QWMP } \\
\text { CS } \\
\text { QWP }\end{array}$ & $\begin{array}{l}\text { CP } \\
\text { CNP } \\
C P\end{array}$ & & $c$ & $\begin{array}{l}645 . \\
646 . \\
646 . \\
6.30\end{array}$ & $\begin{array}{l}1929-31,44- \\
1953 \\
1975-76 \\
1966-72 \\
1960\end{array}$ \\
\hline $\begin{array}{l}\emptyset 73275 \varnothing \emptyset \\
\emptyset 73275 \varnothing \emptyset \\
\varnothing 732749 \varnothing \\
\varnothing 732749 \emptyset \\
6724268 \emptyset\end{array}$ & $\begin{array}{l}\text { Little Washita } R \text { at Ninnekah } \\
\text { Little Washita } R \text { at Ninnekah } \\
\text { Little Washita } R \text { nr Ninnekah } \\
\text { Little Washita } R \text { nr Ninnekah } \\
\text { Little Wewoka Ck nr Wetumka }\end{array}$ & $\begin{array}{l}\text { STR } \\
\text { QWP } \\
\text { STRP } \\
\text { QWD } \\
\text { QWP }\end{array}$ & $\begin{array}{l}\mathrm{CP} \\
\mathrm{CNP} \\
\mathrm{CP}\end{array}$ & & $J G$ & $\begin{array}{l}227 . \\
227 . \\
268 . \\
268 .\end{array}$ & $\begin{array}{l}1952-63 \\
1958-69,61-63 \\
1963-86 \\
1948-56,68-71 \\
1962-63\end{array}$ \\
\hline
\end{tabular}

* Continuous streamflow records for this period.

* Some records in intervening years.

*** Some records may have been collected previously. 
Table 1.--Alphabetical listing of current and historical gaging stations maintained by the U.S. Geological Survey

September 90, 1987--Continued

[Symbols defined on last page of report]

\begin{tabular}{|c|c|c|c|c|c|c|c|}
\hline $\begin{array}{r}\text { Station } \\
\text { Number }\end{array}$ & Station & $\begin{array}{l}\text { Type } \\
\text { of } \\
\text { Data }\end{array}$ & $\begin{array}{l}\text { Q.W. } \\
\text { Para- } \\
\text { meters }\end{array}$ & $\begin{array}{l}\text { Stage } \\
\text { Source }\end{array}$ & $\begin{array}{l}\text { Coop- } \\
\text { erator }\end{array}$ & $\begin{array}{c}\text { Drainage } \\
\text { Area } \\
\left(m i l e^{2}\right)\end{array}$ & Period of Record \\
\hline $\begin{array}{l}\emptyset 72357 \varnothing \emptyset \\
\varnothing 724455 \emptyset \\
\varnothing 71885 \varnothing \emptyset \\
\emptyset 71885 \varnothing \emptyset \\
\varnothing 7190625\end{array}$ & $\begin{array}{l}\text { Little Wolf Ck Trib nr Gage } \\
\text { Longtown Ck nr Enterprise } \\
\text { Lost Ck at SeneCa MO } \\
\text { Lost Ck at Seneca MO } \\
\text { M Fk Big Cabin Ck nr Pyramid Corners }\end{array}$ & $\begin{array}{l}\text { CS } \\
\text { QWP } \\
\text { CS } \\
\text { QWP } \\
\text { QWMP }\end{array}$ & $\begin{array}{l}\mathrm{CP} \\
\mathrm{CP} \\
\mathrm{CP}\end{array}$ & & & $\begin{array}{l}18.4 \\
42.0 \\
42.0 \\
13.4\end{array}$ & $\begin{array}{l}1964-74 \\
1952-53 \\
1949-59 *, 60-74 \\
1951-58 \\
1980-83\end{array}$ \\
\hline $\begin{array}{l}\emptyset 7190625 \\
\emptyset 7196625 \\
\emptyset 7158010 \\
\emptyset 7232029 \\
\emptyset 7232029\end{array}$ & $\begin{array}{l}\text { M Fk Big Cabin Ck nr Pyramid Corners } \\
\text { M Fk Big Cabin Ck nr Pyramid Corners } \\
\text { Main Ck nr Waynoka } \\
\text { Mathuldy Ck nr Crowder } \\
\text { Mathuldy Ck nr Crowder }\end{array}$ & $\begin{array}{l}\text { PR } \\
\text { SED } \\
\text { LF } \\
\text { PR } \\
\text { QWP }\end{array}$ & CNP & & & $\begin{array}{l}13.4 \\
13.4 \\
105 . \\
5.41 \\
5.41\end{array}$ & $\begin{array}{l}1980-83 \\
1980-83 \\
1965-73 \\
1976-81 \\
1976-82\end{array}$ \\
\hline $\begin{array}{l}\varnothing 7232 \varnothing 29 \\
\varnothing 7327449 \\
\varnothing 733391 \varnothing \\
\varnothing 733391 \varnothing \\
\varnothing 733391 \varnothing\end{array}$ & $\begin{array}{l}\text { Mathuldy Ck nr Crowder } \\
\text { McCardo Ck nr Cement } \\
\text { McGee Ck nr Farris } \\
\text { McGee Ck nr Farris } \\
\text { McGee Ck nr Farris }\end{array}$ & $\begin{array}{l}\text { SED } \\
\text { QWP } \\
\text { STR } \\
\text { QWH } \\
\text { QWP }\end{array}$ & $\begin{array}{l}S \\
C P \\
C P \\
C N P\end{array}$ & & & $\begin{array}{l}5.41 \\
176 \\
176 \\
176\end{array}$ & $\begin{array}{l}1976-81 \\
1968-60 \\
1976-82 \\
1976-82 \\
1976-82\end{array}$ \\
\hline $\begin{array}{l}\varnothing 733391 \emptyset \\
\varnothing 73338 \varnothing \emptyset \\
\varnothing 73338 \varnothing \emptyset \\
\varnothing 73165 \varnothing \emptyset \\
\varnothing 736995 \emptyset\end{array}$ & $\begin{array}{l}\text { McGee Ck nr Farris } \\
\text { McGee Ck nr Stringtown } \\
\text { McGee Ck nr Stringtown } \\
\text { Medicine Bluff Ck nr Lawton } \\
\text { Medicine Ck nr Fort Sill }\end{array}$ & $\begin{array}{l}\text { SED } \\
\text { CS } \\
\text { QWP } \\
\text { STR } \\
\text { QWP }\end{array}$ & $\begin{array}{l}\mathrm{S} \\
\mathrm{CP} \\
\mathrm{CP}\end{array}$ & & & $\begin{array}{l}176 . \\
86.6 \\
86.6 \\
161 .\end{array}$ & $\begin{array}{l}1978-81 \\
* 1955-68,69-75 \\
1956-58 \\
1913-19 \\
1951\end{array}$ \\
\hline $\begin{array}{l}\emptyset 7149302 \\
07149600 \\
07149000 \\
\varnothing 722963 \emptyset \\
6723166 \varnothing\end{array}$ & $\begin{array}{l}\text { Medicine Lodge R nr Driftwood } \\
\text { Medicine Lodge R nr Kiowa, KS } \\
\text { Medicine Lodge R nr Kiowa, KS } \\
\text { Merkle Ck at Norman } \\
\text { Middle Ck nr Carson }\end{array}$ & $\begin{array}{l}\text { QWP } \\
\text { QWH } \\
\text { QWMP } \\
\text { CSR } \\
\text { CS }\end{array}$ & $\begin{array}{l}C P \\
C P \\
C N P\end{array}$ & & $\begin{array}{l}W \\
c\end{array}$ & $\begin{array}{l}903 . \\
903 . \\
7.46\end{array}$ & $\begin{array}{l}1962 \\
1965-67,60-62,73- \\
1976-77 \\
1988- \\
1964-74\end{array}$ \\
\hline $\begin{array}{l}\emptyset 73312 ø \emptyset \\
\varnothing 73312 \varnothing \emptyset \\
\varnothing 7194615 \\
\varnothing 733125 \emptyset \\
\varnothing 733125 \varnothing\end{array}$ & $\begin{array}{l}\text { Mill Ck nr Mill Creek } \\
\text { Mill Ck nr Mill Creek } \\
\text { Mill Ck nr Park Hill } \\
\text { Mill Ck nr Ravia } \\
\text { Mill Ck nr Ravia }\end{array}$ & $\begin{array}{l}\text { LF } \\
\text { QWP } \\
\text { CS } \\
\text { STR } \\
\text { QWP }\end{array}$ & $\mathrm{CP}$ & & HG & $\begin{array}{l}46.4 \\
46.4 \\
2.57 \\
89.2 \\
89.2\end{array}$ & $\begin{array}{l}1952-55,68-71 \\
1952-55,60 \\
1965-85 \\
1969-71 \\
1969\end{array}$ \\
\hline $\begin{array}{l}\emptyset 7178 \varnothing 4 \varnothing \\
\varnothing 717864 \varnothing \\
\varnothing 724862 \emptyset \\
\varnothing 724862 \emptyset \\
\varnothing 724862 \emptyset\end{array}$ & $\begin{array}{l}\text { Mingo } C k \text { at } N 46 \text { th } S t \text { at Tulsa } \\
\text { Mingo } C k \text { at } N 46 \text { th } S t \text { at Tulsa } \\
\text { Morris } C k \text { at Howe } \\
\text { Morris } C k \text { at Howe } \\
\text { Morris } C k \text { at Howe }\end{array}$ & $\begin{array}{l}\text { STR } \\
\text { QWH } \\
\text { STR } \\
\text { QWP } \\
\text { SED }\end{array}$ & $\begin{array}{l}P \\
\text { CNP } \\
S\end{array}$ & & $\begin{array}{l}P \\
P\end{array}$ & $\begin{array}{l}19.4 \\
19.4 \\
19.4\end{array}$ & $\begin{array}{l}1987- \\
1987- \\
1978-81 \\
1978-81 \\
1978-81\end{array}$ \\
\hline $\begin{array}{l}\varnothing 733878 \emptyset \\
\varnothing 7339 \varnothing 1 \varnothing \\
\varnothing 73396 \varnothing \emptyset \\
\varnothing 733884 \varnothing \\
\varnothing 73167 \varnothing \varnothing\end{array}$ & $\begin{array}{l}\text { Mountain Fk Trib nr Smithville } \\
\text { Mountain Fk blw Eagletown } \\
\text { Mountain Fk nr Eagletown } \\
\text { Mountain Fk nr Smithville } \\
\text { Mud Ck nr Courtney }\end{array}$ & $\begin{array}{l}\text { CS } \\
\text { QWP } \\
\text { STRP } \\
\text { QWMP } \\
\text { STR }\end{array}$ & $\begin{array}{l}\mathrm{CP} \\
\mathrm{CNP}\end{array}$ & DAR & $\begin{array}{l}\text { HG } \\
\mathrm{C} \\
\mathrm{JG}\end{array}$ & $\begin{array}{l}0.85 \\
787 . \\
672 .\end{array}$ & $\begin{array}{l}1965-86 \\
1961-63 \\
1924-25,29- \\
1976-79 \\
1960-\end{array}$ \\
\hline
\end{tabular}

* Continuous streamflow records for this period.

* Some records in intervening years.

*** Some records may have been collected previously. 
Table 1.--Alphabetica' listing of current and historical gaging stations maintained by the U.S. Geological Survey

September 90, 1987--Continued

[Symbols defined on last page of report]

\begin{tabular}{|c|c|c|c|c|c|c|c|}
\hline $\begin{array}{c}\text { Station } \\
\text { Number }\end{array}$ & Station & $\begin{array}{l}\text { Type } \\
\text { of } \\
\text { Data }\end{array}$ & $\begin{array}{l}\text { Q.W. } \\
\text { Para- } \\
\text { meters }\end{array}$ & $\begin{array}{l}\text { Stage } \\
\text { Source }\end{array}$ & $\begin{array}{l}\text { Coop- } \\
\text { erator }\end{array}$ & $\begin{array}{c}\text { Orainage } \\
\text { Area } \\
(\mathrm{mile})\end{array}$ & Period of Record \\
\hline $\begin{array}{l}\emptyset 73157 \varnothing \emptyset \\
\varnothing 73167 \varnothing \emptyset \\
\varnothing 7315697 \\
\varnothing 733296 \emptyset \\
\varnothing 733296 \varnothing\end{array}$ & $\begin{array}{l}\text { Mud Ck nr Courtney } \\
\text { Mud Ck nr Courtney } \\
\text { Mud Ck nr Grady } \\
\text { Muddy Boggy Ck at Atoka } \\
\text { Muddy Boggy Ck at Atoka }\end{array}$ & $\begin{array}{l}\text { QWP } \\
\text { QWMP } \\
\text { QWP } \\
\text { STR } \\
\text { SED }\end{array}$ & $\begin{array}{l}\text { CP } \\
C N P \\
C P \\
S\end{array}$ & & & $\begin{array}{l}572 . \\
572 . \\
445 \\
445\end{array}$ & $\begin{array}{l}1960,62-63 \\
1975-79 \\
1961-52,60-61 \\
1978-81 \\
1978-81\end{array}$ \\
\hline $\begin{array}{l}\emptyset 733296 \varnothing \\
\varnothing 733276 \varnothing \\
\varnothing 7334 \varnothing \varnothing \varnothing \\
\varnothing 7334 \varnothing \varnothing \emptyset \\
\varnothing 7334 \varnothing \varnothing \varnothing\end{array}$ & $\begin{array}{l}\text { Muddy Boggy Ck at Atoka } \\
\text { Muddy Boggy Ck nr Coalgate } \\
\text { Muddy Boggy Ck nr Farris } \\
\text { Muddy Boggy Ck nr Farris } \\
\text { Muddy Boggy Ck nr Farris }\end{array}$ & $\begin{array}{l}\text { QWP } \\
\text { QWP } \\
\text { STRP } \\
\text { QWP } \\
\text { QWMP }\end{array}$ & $\begin{array}{l}\text { CNP } \\
\text { CP } \\
\text { CP } \\
\text { CNP }\end{array}$ & & C & $\begin{array}{l}446 . \\
1087 \\
1087 \\
1887\end{array}$ & $\begin{array}{l}1978-81 \\
1962 \\
1937- \\
1948,50-58,62-64 \\
1973-79\end{array}$ \\
\hline $\begin{array}{l}\emptyset 73327 \varnothing \varnothing \\
\varnothing 73353 \varnothing \emptyset \\
\varnothing 733536 \varnothing \\
\varnothing 724558 \emptyset \\
\varnothing 724558 \emptyset\end{array}$ & $\begin{array}{l}\text { Muddy Boggy Ck nr Parker } \\
\text { Muddy Boggy Ck nr Unger } \\
\text { Muddy Boggy Ck nr Unger } \\
\text { Mule Ck at SR } 31 \mathrm{nr} \text { McCurtain } \\
\text { Mule Ck at SR } 31 \mathrm{nr} \text { McCurtain }\end{array}$ & $\begin{array}{l}\text { LF } \\
\text { STR } \\
\text { QWP } \\
\text { QWP } \\
\text { PR }\end{array}$ & $\begin{array}{l}C P \\
C P\end{array}$ & OBS & $J G$ & 174. & $\begin{array}{l}1958-73 \\
1982- \\
1962 \\
1981-82 \\
1981-82\end{array}$ \\
\hline $\begin{array}{l}\emptyset 729972 \varnothing \\
\varnothing 724659 \emptyset \\
\varnothing 724559 \emptyset \\
\varnothing 7245592 \\
\varnothing 7246592\end{array}$ & $\begin{array}{l}\text { Mule Ck nr Eldorado } \\
\text { Mule Ck nr MeCurtain } \\
\text { Mule Ck nr McCurtain } \\
\text { Mule Ck nr McCurtain } \\
\text { Mule Ck nr McCurtain }\end{array}$ & $\begin{array}{l}\text { CS } \\
\text { QWP } \\
\text { PR } \\
\text { QWP } \\
\text { PR }\end{array}$ & $\begin{array}{l}\mathrm{CP} \\
\mathrm{CP}\end{array}$ & & & 3.84 & $\begin{array}{l}1965-72 \\
1979-82 \\
1979-82 \\
1981-82 \\
1981-82\end{array}$ \\
\hline $\begin{array}{l}\emptyset 7246594 \\
\emptyset 7246594 \\
\varnothing 7160290 \\
67166290 \\
\varnothing 7333060\end{array}$ & $\begin{array}{l}\text { Mule Ck nr McCurtain } \\
\text { Mule Ck nr McCurtain } \\
N \text { Boggy Ck at Garriott Rd, Enid } \\
N \text { Boggy Ck at Garriott Rd, Enid } \\
N \text { Boggy Ck nr Stringtown }\end{array}$ & $\begin{array}{l}\text { QWP } \\
\text { PR } \\
\text { STR } \\
\text { QWP } \\
\text { STR }\end{array}$ & $\begin{array}{l}\mathrm{CP} \\
\mathrm{BCNP}\end{array}$ & & & $\begin{array}{l} \\
8.98 \\
8.98 \\
136 .\end{array}$ & $\begin{array}{l}1981-82 \\
1981-82 \\
1976 \\
1976 \\
1956-59\end{array}$ \\
\hline 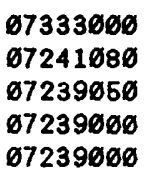 & $\begin{array}{l}N \text { Boggy Ck } n r \text { Stringtown } \\
N \text { Canadian } R \text { Trib at } N W 10 \text {, OKC } \\
N \text { Canadian } R \text { Trib nr Eagle City } \\
N \text { Canadian } R \text { at Canton } \\
N \text { Canadian } R \text { at Canton }\end{array}$ & $\begin{array}{l}\text { QWP } \\
\text { CS } \\
\text { CS } \\
\text { STR } \\
\text { QWD }\end{array}$ & $C P$ & $D C P$ & $\mathrm{C}$ & $\begin{array}{l}136 . \\
1.78 \\
0.52 \\
12484 \\
12484\end{array}$ & $\begin{array}{l}1966-59 \\
1976-81 \\
1964-75 \\
1937- \\
1951-59\end{array}$ \\
\hline $\begin{array}{l}\varnothing 723900 \varnothing \\
\varnothing 7239 \varnothing \varnothing \emptyset \\
\varnothing 723976 \varnothing \\
\varnothing 723750 \varnothing \\
\varnothing 7237500\end{array}$ & $\begin{array}{l}N \text { Canadian } R \text { at Canton } \\
N \text { Canadian } R \text { at Canton } \\
N \text { Canadian } R \text { at Morgan Rd nr Yukon } \\
N \text { Canadian } R \text { at Woodward } \\
N \text { Canadian } R \text { at Woodward }\end{array}$ & $\begin{array}{l}\text { QWMP } \\
\text { SED } \\
\text { QWP } \\
\text { STR } \\
\text { QWP }\end{array}$ & $\begin{array}{l}\text { CNP } \\
\text { S } \\
\text { CNOP } \\
\text { BCNP }\end{array}$ & DCP & $\begin{array}{l}C \\
A\end{array}$ & $\begin{array}{l}12484 \\
12484 \\
11589 \\
11689\end{array}$ & $\begin{array}{l}1973-79 \\
1976 \\
1973 \\
1938- \\
* * 1965,61-63,75-\end{array}$ \\
\hline 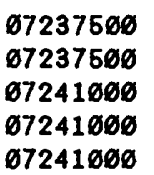 & $\begin{array}{l}N \text { Canadian } R \text { at Woodward } \\
N \text { Canadian } R \text { at Woodward } \\
N \text { Canadian } R \text { blw Lake Ovrholsr nr OKC } \\
N \text { Canadian } R \text { blw Lake Ovrholsr nr OKC } \\
N \text { Canadian } R \text { blw Lake Ovrholsr nr OKC }\end{array}$ & $\begin{array}{l}\text { QWMP } \\
\text { SED } \\
\text { STR } \\
\text { QWD } \\
\text { SED }\end{array}$ & $\begin{array}{l}\text { CNP } \\
\mathrm{S}\end{array}$ & $O C P$ & $\begin{array}{l}A \\
K G\end{array}$ & $\begin{array}{l}11589 \\
11589 \\
13222 \\
13222 \\
13222\end{array}$ & $\begin{array}{l}1973-77 \\
1975- \\
* * 1952-68,73- \\
1968-61 \\
1978-\end{array}$ \\
\hline
\end{tabular}

* Continuous streamflow records for this period.

** Some records in intervening years.

*** Some records may have been collected previously. 
Table 1.--Alphabetical listing of current and historical gaging stations maintained by the U.S. Geological Survey

September 30, 1987--Continued

[Symbols defined on last page of report]

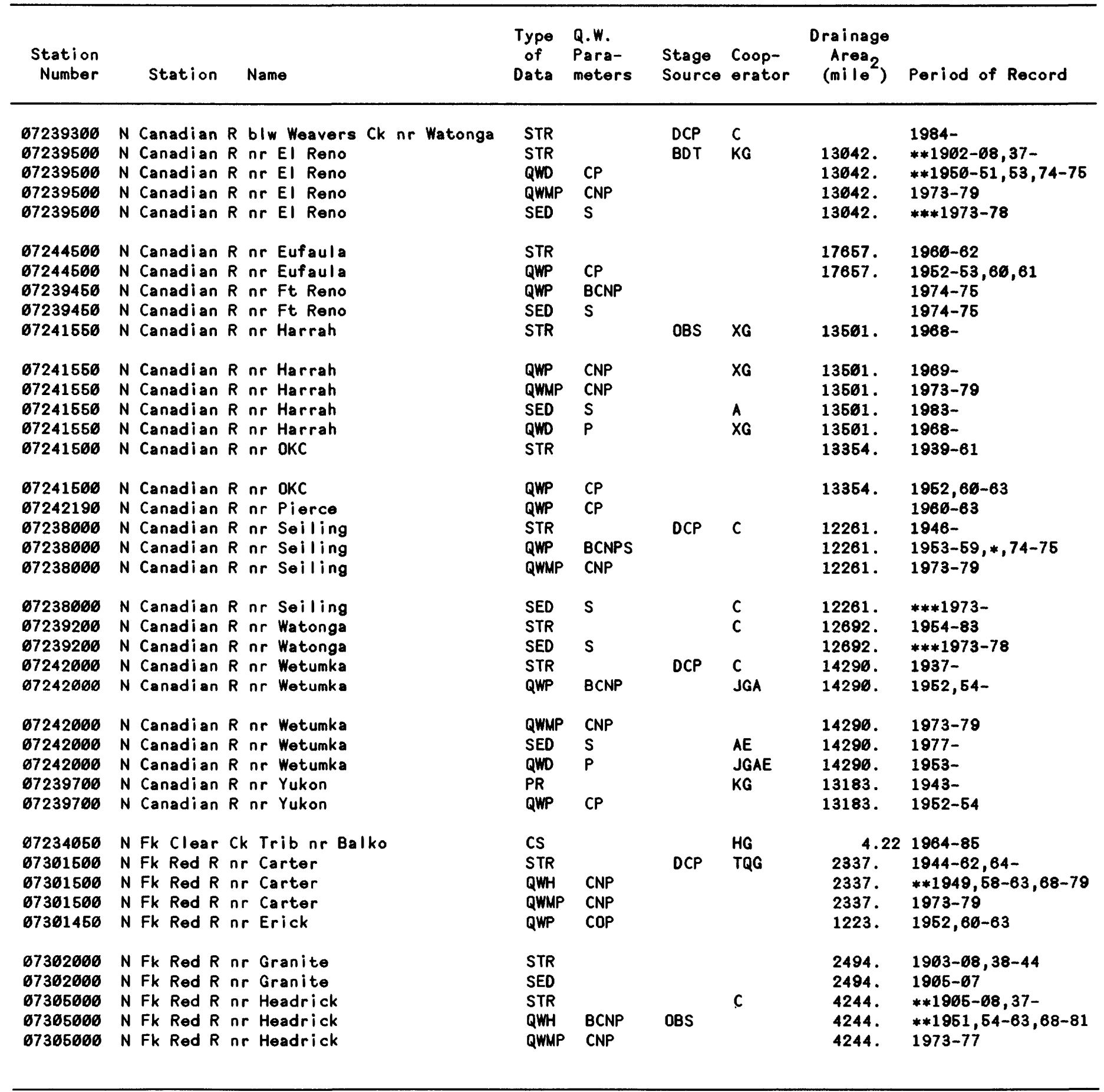

* Continuous streamflow records for this period.

** Some records in intervening years.

*** Some records may have been collected previously. 
Table 1.--Alphabetical listing of current and historical gaging stations maintained by the U.S. Geological Survey

September 30, 1987--Continued

[Symbols defined on last page of report]

\begin{tabular}{|c|c|c|c|c|c|c|c|}
\hline $\begin{array}{r}\text { Station } \\
\text { Number }\end{array}$ & Station & $\begin{array}{l}\text { Type } \\
\text { of } \\
\text { Data }\end{array}$ & $\begin{array}{l}\text { Q.W. } \\
\text { Para- } \\
\text { meters }\end{array}$ & $\begin{array}{l}\text { Stage } \\
\text { Source }\end{array}$ & $\begin{array}{l}\text { Coop- } \\
\text { erator }\end{array}$ & $\begin{array}{c}\text { Drainage } \\
\text { Area } \\
(\mathrm{mile})\end{array}$ & Period of Record \\
\hline 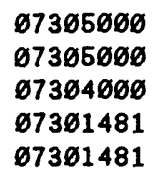 & $\begin{array}{l}\text { N Fk Red R nr Headrick } \\
\text { N Fk Red R nr Headrick } \\
\text { N Fk Red R nr Lugert } \\
\text { N Fk Red R nr Sayre } \\
\text { N Fk Red R nr Sayre }\end{array}$ & $\begin{array}{l}\text { SED } \\
\text { QWP } \\
\text { STR } \\
\text { STR } \\
\text { STGU }\end{array}$ & $\begin{array}{l}S \\
\text { BCNP }\end{array}$ & OBS & $\hat{A}$ & $\begin{array}{l}4244 . \\
4244 . \\
3435 . \\
2169 . \\
2169 .\end{array}$ & $\begin{array}{l}* * * 1973- \\
1982- \\
1936-31 \\
1978-83 \\
1984-\end{array}$ \\
\hline 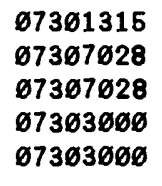 & $\begin{array}{l}\text { N Fk Red R nr Texola } \\
N \text { Fk Red R } n r \text { Tipton } \\
N \text { Fk Red R } n r \text { Tipton } \\
\text { N Fork Red } R \text { blw Lake Altus } n r \text { Lugert } \\
\text { N Fork Red R blw Lake Altus nr Lugert }\end{array}$ & $\begin{array}{l}\text { QWMP } \\
\text { STR } \\
\text { QWP } \\
\text { STR } \\
\text { QWP }\end{array}$ & $\begin{array}{l}\mathrm{CNP} \\
\mathrm{CP} \\
\mathrm{CP}\end{array}$ & OBS & $\begin{array}{l}J \\
\text { TQG }\end{array}$ & $\begin{array}{l}1284 . \\
4681 . \\
4681 \\
2516 \\
2515\end{array}$ & $\begin{array}{l}1977 \\
1983- \\
196 \emptyset \\
* * 1936-32,64- \\
1963\end{array}$ \\
\hline 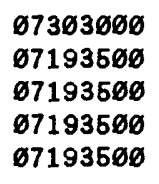 & $\begin{array}{l}\text { N Fork Red R blw Lake Altus } n r \text { Lugert } \\
\text { Neosho } R \text { blw Ft Gibson L nr Ft Gibson } \\
\text { Neosho R blw Ft Gibson L } n r \text { Ft Gibson } \\
\text { Neosho R blw Ft Gibson L nr Ft Gibson } \\
\text { Neosho R blw Ft Gibson L nr Ft Gibson }\end{array}$ & $\begin{array}{l}\text { QWMP } \\
\text { STGU } \\
\text { QWP } \\
\text { QWMP } \\
\text { SED }\end{array}$ & $\begin{array}{l}\text { CNP } \\
\text { BCNP } \\
\text { CNP } \\
S\end{array}$ & OBS & $\begin{array}{l}C \\
A \\
A\end{array}$ & $\begin{array}{l}2515 \\
12495 \\
12495 \\
12495 \\
12495\end{array}$ & $\begin{array}{l}1973-74 \\
1950- \\
1952- \\
1973-77 \\
1973-\end{array}$ \\
\hline $\begin{array}{l}\emptyset 71916 \varnothing \emptyset \\
\varnothing 71915 \emptyset \emptyset \\
\varnothing 71915 \emptyset \emptyset \\
\varnothing 7185 \varnothing \emptyset \emptyset \\
\varnothing 7185 \varnothing \emptyset \emptyset\end{array}$ & $\begin{array}{l}\text { Neosho } R n r \text { Chouteau } \\
\text { Neosho } R n r \text { Chouteau } \\
\text { Neosho } R n r \text { Chouteau } \\
\text { Neosho } R n r \text { Commerce } \\
\text { Neosho } R n r \text { Commerce }\end{array}$ & $\begin{array}{l}\text { STR } \\
\text { QWP } \\
\text { QWMP } \\
\text { STR } \\
\text { QWP }\end{array}$ & $\begin{array}{l}\text { CP } \\
\text { CNP } \\
\text { CNP }\end{array}$ & $D C P$ & LG & $\begin{array}{l}11646 \\
11546 \\
11546 \\
5876 \\
5876\end{array}$ & $\begin{array}{l}1938-56,63- \\
1951-58,60 \\
1973-79 \\
1939- \\
1948-54,60-73\end{array}$ \\
\hline $\begin{array}{l}\emptyset 7185 \varnothing \emptyset \emptyset \\
\emptyset 7185 \varnothing \emptyset \emptyset \\
\emptyset 71895 \varnothing \emptyset \\
\emptyset 719 \varnothing 5 \varnothing \emptyset \\
\emptyset 719 \varnothing 5 \varnothing \emptyset\end{array}$ & $\begin{array}{l}\text { Neosho } R n r \text { Commerce } \\
\text { Neosho } R n r \text { Commerce } \\
\text { Neosho } R n r \text { Grove } \\
\text { Neosho } R n r \text { Langley } \\
\text { Neosho } R n r \text { Langley }\end{array}$ & $\begin{array}{l}\text { SED } \\
\text { QWMP } \\
\text { STR } \\
\text { STR } \\
\text { QWP }\end{array}$ & $\begin{array}{l}S \\
\text { CNP }\end{array}$ & & LG & $\begin{array}{r}5876 . \\
5876 \\
9969 . \\
16336 \\
10335\end{array}$ & $\begin{array}{l}1978- \\
1973-79 \\
1925-39 \\
1939- \\
1950-59\end{array}$ \\
\hline $\begin{array}{l}\emptyset 719 ø 5 \emptyset \emptyset \\
\emptyset 71926 \varnothing \emptyset \\
\varnothing 71925 \varnothing \emptyset \\
\varnothing 717862 \emptyset \\
\varnothing 717862 \emptyset\end{array}$ & $\begin{array}{l}\text { Neosho } R n r \text { Langley } \\
\text { Neosho } R n r \text { Wagoner } \\
\text { Neosho } R n r \text { Wagoner } \\
\text { Newt Graham L D nr Inola } \\
\text { Newt Graham L D nr Inola }\end{array}$ & $\begin{array}{l}\text { QWMP } \\
\text { STR } \\
\text { QWP } \\
\text { QWP } \\
\text { QWMP }\end{array}$ & $\begin{array}{l}\text { CNP } \\
\text { CP } \\
\text { BCNP } \\
\text { CNP }\end{array}$ & & $A$ & $\begin{array}{l}16336 \\
12307 \\
12307 \\
8630 \\
8630\end{array}$ & $\begin{array}{l}1973-79 \\
1924-49 \\
1948-5 \emptyset \\
1971- \\
1973-77\end{array}$ \\
\hline $\begin{array}{l}\emptyset 717862 \emptyset \\
\varnothing 717862 \emptyset \\
\emptyset 7178625 \\
\emptyset 731285 \emptyset \\
\emptyset 731285 \emptyset\end{array}$ & $\begin{array}{l}\text { Newt Graham L D nr Inola } \\
\text { Newt Graham L D nr Inola } \\
\text { Newt Graham L D nr Inola } \\
\text { Nine Mile Beaver Ck nr Elgin } \\
\text { Nine Mile Beaver Ck nr Elgin }\end{array}$ & $\begin{array}{l}\text { SED } \\
\text { RESU } \\
\text { STGU } \\
\text { CSR } \\
\text { CS }\end{array}$ & $S$ & $D C P$ & $\begin{array}{l}A \\
C \\
C \\
H G \\
H G\end{array}$ & $\begin{array}{l}8030 . \\
8030 . \\
6.29 \\
6.29\end{array}$ & $\begin{array}{l}1974- \\
1986- \\
1986- \\
1964-76 \\
1964-\end{array}$ \\
\hline $\begin{array}{l}\emptyset 731285 \emptyset \\
\emptyset 71713 \varnothing \emptyset \\
\emptyset 72332 \emptyset \emptyset \\
\emptyset 716 \varnothing 652 \\
\emptyset 73665 \varnothing \varnothing\end{array}$ & $\begin{array}{l}\text { Nine Mile Beaver Ck nr Elgin } \\
\text { Oologah Lake nr Dologah } \\
\text { Optima Lake nr Hardesty } \\
\text { Osage Ck nr Medford } \\
\text { Otter Ck at Mt Park }\end{array}$ & $\begin{array}{l}\text { CS } \\
\text { RES } \\
\text { RES } \\
\text { QWP } \\
\text { STR }\end{array}$ & $C P$ & $D C P$ & $\begin{array}{l}\mathrm{HG} \\
\mathrm{C} \\
\mathrm{C}\end{array}$ & $\begin{array}{l}6.29 \\
4339 . \\
6029 . \\
164 .\end{array}$ & $\begin{array}{l}1964-85 \\
1963- \\
1978- \\
1962 \\
1946-61\end{array}$ \\
\hline
\end{tabular}

* Continuous streamflow records for this period.

** Some records in intervening years.

*** Some records may have been collected previously. 
Table 1.--Alphabetical listing of current and historical gaging stations maintained by the U.S. Geological Survey

September 30 , 1987--Continued

[Symbols defined on last page of report]

\begin{tabular}{|c|c|c|c|c|c|c|c|}
\hline $\begin{array}{c}\text { Station } \\
\text { Number }\end{array}$ & Station Name & $\begin{array}{l}\text { Type } \\
\text { of } \\
\text { Data }\end{array}$ & $\begin{array}{l}\text { Q.W. } \\
\text { Para- } \\
\text { meters }\end{array}$ & $\begin{array}{l}\text { Stage } \\
\text { Source }\end{array}$ & $\begin{array}{l}\text { Coop- } \\
\text { erator }\end{array}$ & $\begin{array}{c}\text { Drainage } \\
\text { Area } \\
\left(m i l e^{2}\right)\end{array}$ & Period of Record \\
\hline $\begin{array}{l}\varnothing 73 \varnothing 66 \varnothing \emptyset \\
\varnothing 73 \varnothing 7 \varnothing 1 \varnothing \\
\varnothing 73 \varnothing 7 \varnothing 1 \varnothing \\
\varnothing 717868 \emptyset \\
\varnothing 73 \varnothing 7 \varnothing 2 \varnothing\end{array}$ & $\begin{array}{l}\text { Otter Ck at Mt Park } \\
\text { Otter Ck nr Snyder } \\
\text { Otter Ck nr Snyder } \\
\text { Otter Ck nr Tiawah } \\
\text { Otter Ck nr Tipton }\end{array}$ & $\begin{array}{l}\text { QWP } \\
\text { QWP } \\
\text { STGU } \\
\text { CS } \\
\text { QWP }\end{array}$ & $\begin{array}{l}C P \\
C P \\
C P\end{array}$ & $D C P$ & C & 16.2 & $\begin{array}{l}1962 \\
1961,60-63 \\
1984- \\
1966-72 \\
1960-63\end{array}$ \\
\hline $\begin{array}{l}\emptyset 72491 \varnothing \emptyset \\
\varnothing 72491 \varnothing \emptyset \\
\varnothing 72491 \varnothing \emptyset \\
\varnothing 72337 \varnothing \emptyset \\
\varnothing 72337 \varnothing \emptyset\end{array}$ & $\begin{array}{l}\text { Owl Ck nr McCurtain } \\
\text { Owl Ck nr McCurtain } \\
\text { Owl Ck nr McCurtain } \\
\text { Palo Duro Ck nr Range } \\
\text { Palo Duro Ck nr Range }\end{array}$ & $\begin{array}{l}\text { STR } \\
\text { QWP } \\
\text { SED } \\
\text { LF } \\
\text { QWP }\end{array}$ & $\begin{array}{l}\text { CNP } \\
S \\
\text { CNP }\end{array}$ & & & $\begin{array}{r}27.9 \\
27.9 \\
27.9 \\
1745 . \\
1746 .\end{array}$ & $\begin{array}{l}1978-81 \\
1978-81 \\
1978-81 \\
1951-73 \\
1952-62\end{array}$ \\
\hline $\begin{array}{l}\emptyset 72328 \emptyset \emptyset \\
\emptyset 723199 \emptyset \\
\varnothing 723199 \emptyset \\
\emptyset 723199 \emptyset \\
\emptyset 730820 \emptyset\end{array}$ & $\begin{array}{l}\text { Panhandle Univ. - NADP at Goodwell } \\
\text { Peaceable Ck nr Haileyville } \\
\text { Peaceable Ck nr Haileyville } \\
\text { Peaceable Ck nr Haileyville } \\
\text { Pease R nr Vernon TX }\end{array}$ & $\begin{array}{l}\text { QWH } \\
\text { STR } \\
\text { QWP } \\
\text { SED } \\
\text { QWP }\end{array}$ & $\begin{array}{l}\text { BCNP } \\
S \\
C\end{array}$ & OBS & A & $\begin{array}{l}134 \\
134 \\
134 \\
3488\end{array}$ & $\begin{array}{l}1986- \\
1978-82 \\
1978-82 \\
1978-82 \\
1960\end{array}$ \\
\hline $\begin{array}{l}\emptyset 724661 \emptyset \\
\varnothing 73313 \varnothing \emptyset \\
\varnothing 73313 \varnothing \emptyset \\
\varnothing 733678 \emptyset \\
\varnothing 72377 \varnothing \emptyset\end{array}$ & $\begin{array}{l}\text { Pecan Ck nr Spiro } \\
\text { Pennington Ck nr Reagan } \\
\text { Pennington Ck } n r \text { Reagan } \\
\text { Perry Ck } n r \text { Idabel } \\
\text { Persimmon Ck nr Mutual }\end{array}$ & $\begin{array}{l}\text { CSR } \\
\text { LF } \\
\text { QWP } \\
\text { CS } \\
\text { LF }\end{array}$ & $\mathrm{CP}$ & & & $\begin{array}{l}6.90 \\
65.7 \\
65.7 \\
7.63 \\
164 .\end{array}$ & $\begin{array}{l}1964-76 \\
1951-66,68-73 \\
1951-65,68-59 \\
1965-73 \\
1968-61,65-73\end{array}$ \\
\hline $\begin{array}{l}\emptyset 72377 \varnothing \emptyset \\
\varnothing 73373 \varnothing \emptyset \\
\emptyset 723195 \emptyset \\
\varnothing 71665 \varnothing \emptyset \\
\emptyset 71655 \varnothing \emptyset\end{array}$ & $\begin{array}{l}\text { Persimmon Ck nr Mutual } \\
\text { Pine Ck Lake nr Wright City } \\
\text { Pine Ck nr Higgins } \\
\text { Polecat Ck at Heyburn } \\
\text { Polecat Ck at Heyburn }\end{array}$ & $\begin{array}{l}\text { QWP } \\
\text { RESP } \\
\text { CS } \\
\text { STRP } \\
\text { QWP }\end{array}$ & $C P$ & RES & $\begin{array}{l}\mathrm{C} \\
\mathrm{HG}\end{array}$ & $\begin{array}{l}164 . \\
635 . \\
9.99 \\
123 . \\
123 .\end{array}$ & $\begin{array}{l}1951,58 \\
1969- \\
1964-86 \\
1943-79 \\
1952-53,56-58,61\end{array}$ \\
\hline $\begin{array}{l}\emptyset 71666 \varnothing \emptyset \\
\emptyset 716651 \emptyset \\
\varnothing 716 \varnothing 7 \varnothing \emptyset \\
\emptyset 723262 \emptyset \\
\varnothing 7247 \varnothing \emptyset \emptyset\end{array}$ & $\begin{array}{l}\text { Polecat } C k \text { at Heyburn } \\
\text { Polecat } C k n r \text { Jenks } \\
\text { Pond } C k n r \text { Lamont } \\
\text { Pony } C k n r \text { Optima } \\
\text { Poteau } R \text { at Cauthron AR }\end{array}$ & $\begin{array}{l}\text { SED } \\
\text { QWP } \\
\text { QWP } \\
\text { LF } \\
\text { QWMP }\end{array}$ & $\begin{array}{l}S \\
C O P \\
C P \\
\text { CNP }\end{array}$ & & & $\begin{array}{l}123 . \\
223 . \\
203 .\end{array}$ & $\begin{array}{l}1978-79 \\
1960-63 \\
1961-66,58-69,62 \\
1966-71 \\
1973-76\end{array}$ \\
\hline $\begin{array}{l}\emptyset 7249 \emptyset \emptyset \emptyset \\
\varnothing 7249 \varnothing \varnothing \emptyset \\
\varnothing 7249 \varnothing \varnothing \emptyset \\
\varnothing 7249438 \\
\varnothing 724944 \emptyset\end{array}$ & $\begin{array}{l}\text { Poteau } R \text { at Poteau } \\
\text { Poteau } R \text { at Poteau } \\
\text { Poteau } R \text { at Poteau } \\
\text { Poteau } R \text { nr Braden } \\
\text { Poteau } R \text { nr Fort Smith AR }\end{array}$ & $\begin{array}{l}\text { STR } \\
\text { QWP } \\
\text { STGU } \\
\text { QWP } \\
\text { QWMP }\end{array}$ & $\begin{array}{l}C P \\
C P \\
C N P\end{array}$ & & C & $\begin{array}{l}1240 . \\
1240 . \\
1240 .\end{array}$ & $\begin{array}{l}1938-46 \\
1944-45 \\
1986- \\
1958-59,62-63 \\
1975-77\end{array}$ \\
\hline $\begin{array}{l}\emptyset 724735 \emptyset \\
\emptyset 7249419 \\
\emptyset 7249419 \\
\emptyset 7249419 \\
\emptyset 7249419\end{array}$ & $\begin{array}{l}\text { Poteau R nr Heavener } \\
\text { Poteau R nr Panama } \\
\text { Poteau R nr Panama } \\
\text { Poteau R nr Panama } \\
\text { Poteau R nr Panama }\end{array}$ & $\begin{array}{l}\text { QWMP } \\
\text { CS } \\
\text { QWP } \\
\text { SED } \\
\text { STGU }\end{array}$ & $\begin{array}{l}\text { CNP } \\
\text { CNP } \\
\mathrm{S}\end{array}$ & $D C P$ & $C$ & & $\begin{array}{l}1976-79 \\
1976-79 \\
1976-79 \\
1977-79 \\
1986-\end{array}$ \\
\hline
\end{tabular}

* Continuous streamflow records for this period.

** Some records in intervening years.

*** Some records may have been collected previously. 
Table 1.--Alphabetical listing of current and historical gaging stations maintained by the U.S. Geological Survey

September 30, 1987--Continued

[Symbols defined on last page of report]

\begin{tabular}{|c|c|c|c|c|c|c|c|}
\hline $\begin{array}{r}\text { Station } \\
\text { Number }\end{array}$ & Station & $\begin{array}{l}\text { Type } \\
\text { of } \\
\text { Data }\end{array}$ & $\begin{array}{l}\text { Q.W. } \\
\text { Para- } \\
\text { meters }\end{array}$ & $\begin{array}{l}\text { Stage } \\
\text { Source }\end{array}$ & $\begin{array}{l}\text { Coop- } \\
\text { erator }\end{array}$ & $\begin{array}{c}\text { Drainage } \\
\text { Area } \\
(m i l e)\end{array}$ & Period of Record \\
\hline $\begin{array}{l}\emptyset 72486 \varnothing \emptyset \\
\varnothing 72486 \varnothing \emptyset \\
\varnothing 72486 \varnothing \emptyset \\
\varnothing 72985 \varnothing \emptyset \\
\varnothing 71586 \varnothing \emptyset\end{array}$ & $\begin{array}{l}\text { Poteau R nr Wister } \\
\text { Poteau } R \text { nr Wister } \\
\text { Poteau } R n r \text { Wister } \\
\text { Prairie Dog Town Fk Red R nr Brice TX } \\
\text { Preacher Ck nr Dover }\end{array}$ & $\begin{array}{l}\text { STGU } \\
\text { QWP } \\
\text { QWMP } \\
\text { QWP } \\
\text { CS }\end{array}$ & $\begin{array}{l}\mathrm{CP} \\
\mathrm{CNP} \\
\mathrm{C}\end{array}$ & & $H G$ & $\begin{array}{l}993 . \\
993 . \\
993 . \\
6082 . \\
14.6\end{array}$ & $\begin{array}{l}1938- \\
1948,62,65-69 \\
1973-79 \\
1960 \\
* 1951-57,64-85\end{array}$ \\
\hline $\begin{array}{l}\emptyset 71585 \varnothing \emptyset \\
6715849 \emptyset \\
6719203 \emptyset \\
6719265 \emptyset \\
6719206 \emptyset\end{array}$ & $\begin{array}{l}\text { Preacher Ck nr Dover } \\
\text { Preacher Ck nr Hennessey } \\
\text { Pryor Ck at Elliot St Bridge nr Pryor } \\
\text { Pryor Ck at Hwy 69A Bridge nr Pryor } \\
\text { Pryor Ck blw Sulphur Ck nr Pryor }\end{array}$ & $\begin{array}{l}\text { QWP } \\
\text { QWP } \\
\text { QWP } \\
\text { QWP } \\
\text { QWP }\end{array}$ & $\begin{array}{l}\text { CP } \\
\text { CP } \\
\text { CNP } \\
\text { CP } \\
\text { CNP }\end{array}$ & & & 14.5 & $\begin{array}{l}1952-54 \\
1953 \\
1966-71 \\
1958,62-63 \\
1966-75\end{array}$ \\
\hline 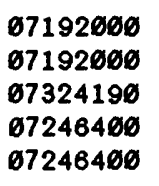 & $\begin{array}{l}\text { Pryor Ck nr Pryor } \\
\text { Pryor Ck nr Pryor } \\
\text { Quartermaster Ck nr Hammon } \\
R S \text { Kerr L D nr Sallisaw (Ark R) } \\
\text { RS Kerr L D nr Sallisaw (Ark R) }\end{array}$ & $\begin{array}{l}\text { STR } \\
\text { QWP } \\
\text { QWP } \\
\text { QWP } \\
\text { QWMP }\end{array}$ & $\begin{array}{l}C P \\
C P \\
C P \\
C N P\end{array}$ & & $J G$ & $\begin{array}{r}229 . \\
229 . \\
147756 . \\
147756 .\end{array}$ & $\begin{array}{l}1948-64 \\
1948-63 \\
197 \varnothing-71 \\
197 \varnothing- \\
1975-79\end{array}$ \\
\hline $\begin{array}{l}\emptyset 724646 \varnothing \\
6724631 \varnothing \\
\varnothing 732636 \varnothing \\
\varnothing 732536 \varnothing \\
\emptyset 715316 \varnothing\end{array}$ & $\begin{array}{l}\text { R S Kerr L D nr Sallisaw (Ark R) } \\
R \text { S Kerr L D nr Sallisaw (Ark US) } \\
\text { Rainy Mtn Ck at Mountain View } \\
\text { Rainy Mtn Ck at Mountain View } \\
\text { Ranch Ck at Cleveland Dam nr Cleveland }\end{array}$ & $\begin{array}{l}\text { RESU } \\
\text { RESU } \\
\text { LF } \\
\text { QWP } \\
\text { STR }\end{array}$ & $\mathrm{CP}$ & DCP & $\begin{array}{l}c \\
c\end{array}$ & $\begin{array}{r}147766 \\
309 . \\
309 . \\
21.9\end{array}$ & $\begin{array}{l}1986- \\
1986- \\
1951-55,58-59 \\
1952-65,58 \\
1945-63\end{array}$ \\
\hline $\begin{array}{l}\emptyset 716310 \emptyset \\
\emptyset 731141 \varnothing \\
\emptyset 724756 \varnothing \\
\emptyset 724765 \varnothing \\
\emptyset 724766 \varnothing\end{array}$ & $\begin{array}{l}\text { Ranch Ck at Cleveland Dam nr Cleveland } \\
\text { Red Ck nr Snyder } \\
\text { Red Oak Ck nr Red Oak } \\
\text { Red Oak Ck nr Red Oak } \\
\text { Red Oak Ck nr Red Oak }\end{array}$ & $\begin{array}{l}\text { QWP } \\
\text { CS } \\
\text { STR } \\
\text { QWP } \\
\text { SED }\end{array}$ & $\begin{array}{l}\text { CNP } \\
S\end{array}$ & & & $\begin{array}{l}21.9 \\
6.12 \\
13.1 \\
13.1 \\
13.1\end{array}$ & $\begin{array}{l}1953 \\
1965-74 \\
1978-82 \\
1978-81 \\
1978-81\end{array}$ \\
\hline $\begin{array}{l}\emptyset 724755 \varnothing \\
\varnothing 73356 \varnothing \emptyset \\
\varnothing 73356 \varnothing \emptyset \\
\varnothing 73356 \varnothing \emptyset \\
\varnothing 73316 \varnothing \varnothing\end{array}$ & $\begin{array}{l}\text { Red Oak Ck nr Red Oak } \\
\text { Red } R \text { at Arthur City TX } \\
\text { Red } R \text { at Arthur City TX } \\
\text { Red } R \text { at Arthur City TX } \\
\text { Red } R \text { at Denison Dam nr Denison TX }\end{array}$ & $\begin{array}{l}\text { QWH } \\
\text { STRP } \\
\text { QWP } \\
\text { QWMP } \\
\text { STRP }\end{array}$ & $\begin{array}{l}P \\
C P \\
C N P\end{array}$ & DAR & $C$ & $\begin{array}{l}13.1 \\
44531 \\
44531 \\
44531 \\
39720\end{array}$ & $\begin{array}{l}1980-81 \\
* * 1936- \\
1960-63 \\
1976-79 \\
1961-\end{array}$ \\
\hline $\begin{array}{l}\varnothing 7331600 \\
67337060 \\
\varnothing 7308500 \\
\varnothing 7332006 \\
67332006\end{array}$ & $\begin{array}{l}\text { Red } R \text { at Denison Dam } n r \text { Denison } T X \\
\text { Red } R \text { at Index AR } \\
\text { Red } R n r \text { Burkburnett } T X \\
\text { Red } R \text { nr Colbert } \\
\text { Red } R \text { nr Colbert }\end{array}$ & $\begin{array}{l}\text { QWMP } \\
\text { QWD } \\
\text { QWMP } \\
\text { STR } \\
\text { QWP }\end{array}$ & $\begin{array}{l}\mathrm{CP} \\
\mathrm{C} \\
\mathrm{CNP} \\
\mathrm{CP}\end{array}$ & & & $\begin{array}{l}39720 \\
48030 \\
20570 \\
39777 \\
39777\end{array}$ & $\begin{array}{l}1973-77 \\
1960-63 \\
1973-77 \\
1924-61 \\
1960-61\end{array}$ \\
\hline $\begin{array}{l}\emptyset 733682 \emptyset \\
\emptyset 7316 \varnothing 0 \varnothing \\
\varnothing 7316 \varnothing \varnothing \emptyset \\
\varnothing 7316 \varnothing 0 \varnothing \\
\varnothing 7299665\end{array}$ & $\begin{array}{l}\text { Red R nr De Kalb TX } \\
\text { Red R nr Gainesville TX } \\
\text { Red R nr Gainesville TX } \\
\text { Red R nr Gainesville TX } \\
\text { Red R nr Hollis }\end{array}$ & $\begin{array}{l}\text { QWMP } \\
\text { STRP } \\
\text { QWP } \\
\text { QWMP } \\
\text { QWMP }\end{array}$ & $\begin{array}{l}\text { CNP } \\
\text { COP } \\
\text { CNP } \\
\text { CNP }\end{array}$ & BDT & $C$ & $\begin{array}{c}47348 . \\
36782 . \\
30782 . \\
30782 . \\
8154 .\end{array}$ & $\begin{array}{l}1973-77 \\
1936- \\
1953-63 \\
1973-77 \\
1977\end{array}$ \\
\hline
\end{tabular}

* Continuous streamflow records for this period.

* Some records in intervening years.

*** Some records may have been collected previously. 
Table 1.--Alphabetical listing of current and historical gaging stations maintained by the U.S. Geological Survey

September 30, 1987--Continued

[Symbols defined on last page of report]

\begin{tabular}{|c|c|c|c|c|c|c|c|}
\hline $\begin{array}{c}\text { Station } \\
\text { Number }\end{array}$ & Station & $\begin{array}{l}\text { Type } \\
\text { of } \\
\text { Data }\end{array}$ & $\begin{array}{l}\text { Q.W. } \\
\text { Para- } \\
\text { meters }\end{array}$ & $\begin{array}{l}\text { Stage } \\
\text { Source }\end{array}$ & $\begin{array}{l}\text { Coop- } \\
\text { erator }\end{array}$ & $\begin{array}{c}\text { Drainage } \\
\text { Area } \\
(\mathrm{mile})\end{array}$ & Period of Record \\
\hline $\begin{array}{l}\emptyset 733676 \emptyset \\
\varnothing 733685 \emptyset \\
\varnothing 729967 \emptyset \\
\emptyset 73156 \varnothing \emptyset \\
\varnothing 733673 \emptyset\end{array}$ & $\begin{array}{l}\text { Red } R n r \text { Millerton } \\
\text { Red } R n r \text { New Boston TX } \\
\text { Red } R n r \text { Quanah TX } \\
\text { Red } R n r \text { Terral } \\
\text { Red } R n r \text { Valliant }\end{array}$ & $\begin{array}{l}\text { QWP } \\
\text { QWP } \\
\text { QWMP } \\
\text { QWMP } \\
\text { QWP }\end{array}$ & $\begin{array}{l}\text { CNP } \\
\text { C } \\
\text { CNP } \\
\text { CNP } \\
\text { CNP }\end{array}$ & & & $\begin{array}{l}46930 . \\
8321 \\
28723 \text {. } \\
46730 \text {. }\end{array}$ & $\begin{array}{l}1971-76 \\
1961-63 \\
1973-77 \\
1973-77 \\
1971-76\end{array}$ \\
\hline $\begin{array}{l}6731272 \varnothing \\
6715236 \emptyset \\
67329843 \\
67329853 \\
6732996 \varnothing\end{array}$ & $\begin{array}{l}\text { Red R nr Waurika } \\
\text { Red Rock nr Red Rock } \\
\text { Rock Ck N of Sulphur } \\
\text { Rock Ck S of Platt N Pk nr Surphur } \\
\text { Rock Ck at Dougherty }\end{array}$ & $\begin{array}{l}\text { QWMP } \\
\text { QWP } \\
\text { QWP } \\
\text { QWP } \\
\text { STR }\end{array}$ & $\begin{array}{l}\text { CNP } \\
\text { CNP } \\
C P \\
C P\end{array}$ & & & 138. & $\begin{array}{l}1978-79 \\
1951-69,62-63 \\
1960,69 \\
1960 \\
1956-67\end{array}$ \\
\hline $\begin{array}{l}6732990 \varnothing \\
6733267 \varnothing \\
6733531 \varnothing \\
67232631 \\
6724907 \varnothing\end{array}$ & $\begin{array}{l}\text { Rock Ck at Dougherty } \\
\text { Rock Ck nr Achille } \\
\text { Rock Ck nr Boswell } \\
\text { Rock Ck nr Crowder } \\
\text { Rock Ck nr Red Oak }\end{array}$ & $\begin{array}{l}\text { QWD } \\
\text { CS } \\
\text { CS } \\
\text { QWP } \\
\text { PR }\end{array}$ & $\begin{array}{l}\mathrm{CP} \\
\mathrm{CNP}\end{array}$ & & HG & $\begin{array}{r}138 . \\
6.72 \\
6.94 \\
12.6\end{array}$ & $\begin{array}{l}1951-60 \\
1965-74 \\
1965-85 \\
1961 \\
1978-81\end{array}$ \\
\hline $\begin{array}{l}6724967 \varnothing \\
6724967 \varnothing \\
67336716 \\
67152416 \\
67165507\end{array}$ & $\begin{array}{l}\text { Rock Ck nr Red Oak } \\
\text { Rock Ck nr Red Oak } \\
\text { Rock Ck nr Sawyer } \\
\text { Rock Ck nr Shidler } \\
\text { Rock Cr at Sapulpa }\end{array}$ & $\begin{array}{l}\text { QWP } \\
\text { SED } \\
\text { CS } \\
\text { CS } \\
\text { LF }\end{array}$ & $\begin{array}{l}\text { CNP } \\
S\end{array}$ & & SG & $\begin{array}{c}12.0 \\
12.0 \\
3.39 \\
9.13 \\
67.3\end{array}$ & $\begin{array}{l}1978-81 \\
1978-81 \\
1964-74 \\
1985-72 \\
1979-85\end{array}$ \\
\hline $\begin{array}{l}6722829 \emptyset \\
673296 \varnothing \varnothing \\
\varnothing 73296 \varnothing \varnothing \\
673295 \varnothing \varnothing \\
\varnothing 73295 \varnothing \varnothing\end{array}$ & $\begin{array}{l}\text { Rough Ck nr Thomas } \\
\text { Rush Ck at Purdy } \\
\text { Rush Ck at Purdy } \\
\text { Rush Ck nr Maysville } \\
\text { Rush Ck nr Maysville }\end{array}$ & $\begin{array}{l}\text { CS } \\
\text { STR } \\
\text { QWP } \\
\text { STR } \\
\text { QWD }\end{array}$ & $\begin{array}{l}\mathrm{CP} \\
\mathrm{CP}\end{array}$ & OBS & $\begin{array}{c}H G \\
J G\end{array}$ & $\begin{array}{l}10.4 \\
145 \\
145 \\
206 \\
206\end{array}$ & $\begin{array}{l}1964-85 \\
1946-63,82- \\
1947-54,58 \\
1953-76 \\
1954-71\end{array}$ \\
\hline $\begin{array}{l}\varnothing 73295 \varnothing \varnothing \\
\varnothing 73295 \varnothing \varnothing \\
\varnothing 732955 \varnothing \\
673287 \varnothing \varnothing \\
67241631\end{array}$ & $\begin{array}{l}\text { Rush Ck nr Maysville } \\
\text { Rush Ck nr Maysville } \\
\text { Rush Ck nr Pauls Valley } \\
\text { Rush Ck nr Rush Springs } \\
\text { S Br T, T2 Mst'g Ck at Sw74, Mustang }\end{array}$ & $\begin{array}{l}\text { SED } \\
\text { CS } \\
\text { QWP } \\
\text { LF } \\
\text { CSR }\end{array}$ & $\begin{array}{l}\mathrm{S} \\
\mathrm{CP}\end{array}$ & & JG & $\begin{array}{l}206 . \\
206 .\end{array}$ & $\begin{array}{l}1976 \\
1983- \\
1952-53,58-59 \\
1952 \\
1976-81\end{array}$ \\
\hline $\begin{array}{l}6724163 \varnothing \\
6719135 \varnothing \\
6724556 \varnothing \\
6724556 \varnothing \\
672456 \varnothing \varnothing\end{array}$ & $\begin{array}{l}\text { S Br T2 Mst'g Ck at Sw74, Mustang } \\
\text { Salina Ck nr Salina } \\
\text { Sallisaw Ck nr Sallisaw } \\
\text { Sallisaw Ck nr Sallisaw } \\
\text { Sallisaw Ck nr Sallisaw }\end{array}$ & $\begin{array}{l}\text { CSR } \\
\text { QWP } \\
\text { STRP } \\
\text { QWP } \\
\text { QWMP }\end{array}$ & $\begin{array}{l}\mathrm{CP} \\
\mathrm{CP} \\
\mathrm{CNP}\end{array}$ & & & $\begin{array}{l}0.54 \\
182 . \\
182 . \\
182 .\end{array}$ & $\begin{array}{l}1976-81 \\
1948-49,51-53,58-69 \\
1942-76 \\
1952-63 \\
1977\end{array}$ \\
\hline $\begin{array}{l}\emptyset 715818 \emptyset \\
\varnothing 717123 \emptyset \\
\varnothing 72368 \varnothing \emptyset \\
\varnothing 723 ø 8 \varnothing \emptyset \\
\varnothing 715815 \emptyset\end{array}$ & $\begin{array}{l}\text { Salt Ck Trib nr Okeene } \\
\text { Salt Ck nr Alluwe } \\
\text { Salt Ck nr Dewright } \\
\text { Salt Ck nr Dewright } \\
\text { Salt Ck nr Hitchcock }\end{array}$ & $\begin{array}{l}\text { CS } \\
\text { QWP } \\
\text { STR } \\
\text { QWP } \\
\text { STR }\end{array}$ & $\begin{array}{l}\mathrm{CP} \\
\mathrm{CP}\end{array}$ & & & $\begin{array}{l}8.23 \\
216 . \\
210 . \\
44.4\end{array}$ & $\begin{array}{l}1964-74 \\
1952-53,59 \\
1960-63,66-67 \\
1952-63 \\
1960,68-70\end{array}$ \\
\hline
\end{tabular}

* Continuous streamflow records for this period.

* Some records in intervening years.

*** Some records may have been collected previously. 
Table 1.--Alphabetical listing of current and historical gaging stations maintained by the U.S. Geological Survey

September 90, 1987--Continued

[Symbols defined on last page of report]

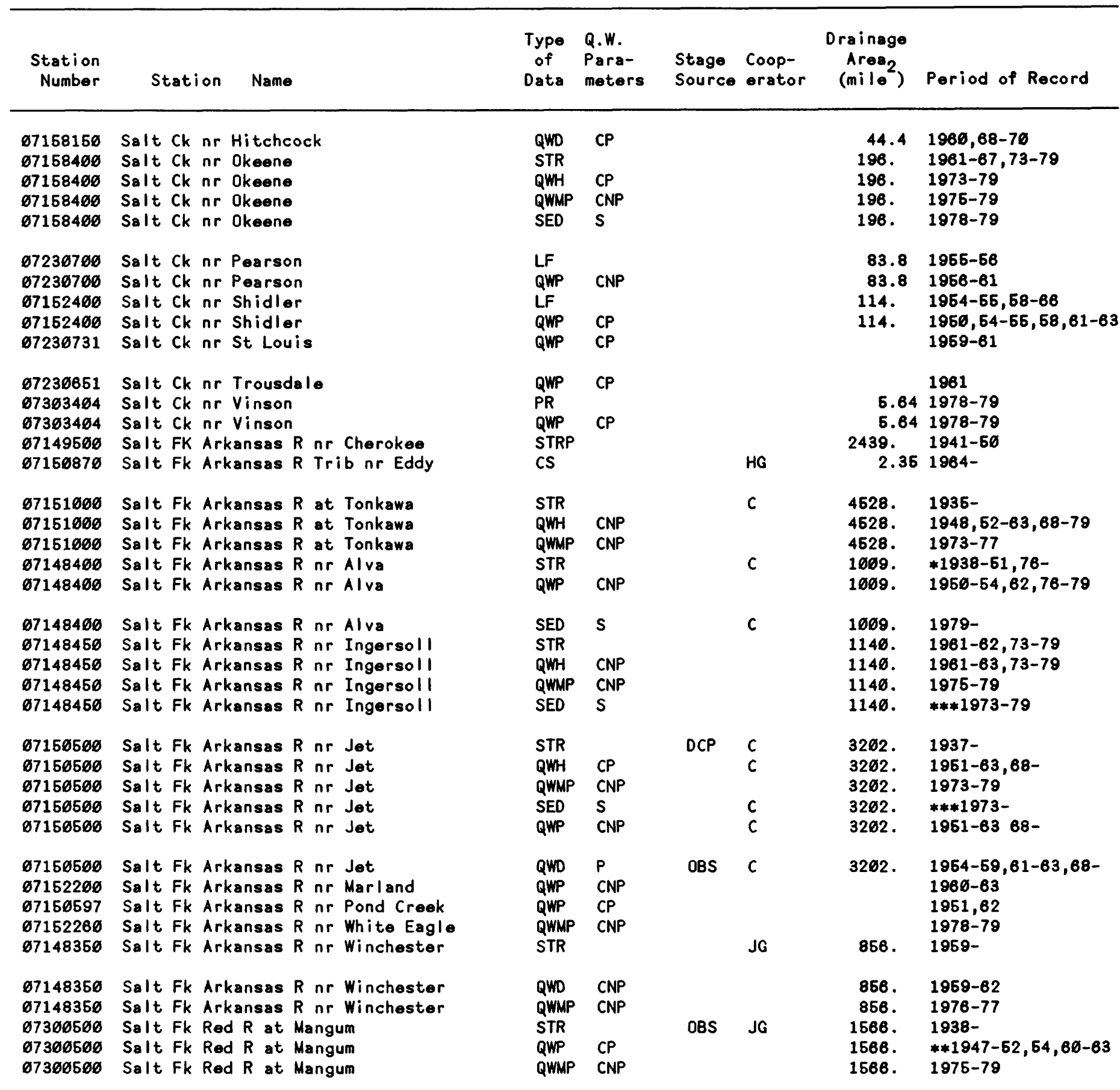

* Continuous streamflow records for this period.

** Some records in intervening years.

*** Some records may have been collected previously. 
Table 1.--Alphabetical listing of current and historical gaging stations maintained by the U.S. Geological Survey

September So, 1987--Continued

[Symbols defined on last page of report]

\begin{tabular}{|c|c|c|c|c|c|c|c|}
\hline $\begin{array}{l}\text { Station } \\
\text { Number }\end{array}$ & Station & $\begin{array}{l}\text { Type } \\
\text { of } \\
\text { Data }\end{array}$ & $\begin{array}{l}\text { Q.W. } \\
\text { Para- } \\
\text { meters }\end{array}$ & $\begin{array}{l}\text { Stage } \\
\text { Source }\end{array}$ & $\begin{array}{l}\text { Coop- } \\
\text { erator }\end{array}$ & $\begin{array}{c}\text { Drainage } \\
\text { Area } \\
\left(m i l e^{2}\right)\end{array}$ & Period of Record \\
\hline 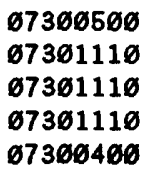 & $\begin{array}{l}\text { Salt Fk Red } R \text { at Mangum } \\
\text { Salt Fk Red } R \text { nr Elmer } \\
\text { Salt Fk Red } R \text { nr Elmer } \\
\text { Salt Fk Red R nr Elmer } \\
\text { Salt Fk Red R nr Vinson }\end{array}$ & $\begin{array}{l}\text { SED } \\
\text { STR } \\
\text { QWP } \\
\text { SED } \\
\text { QWP }\end{array}$ & $\begin{array}{l}S \\
\text { BCNP } \\
S \\
\text { CP }\end{array}$ & OBS & $\begin{array}{l}J G \\
A \\
A\end{array}$ & $\begin{array}{l}1566 . \\
1878 . \\
1878 \\
1878 . \\
1421\end{array}$ & $\begin{array}{l}1976-78 \\
1979- \\
1978- \\
1978- \\
1952-54,60-63\end{array}$ \\
\hline $\begin{array}{l}\emptyset 73 ø 64 \varnothing \emptyset \\
\varnothing 736 \varnothing \varnothing \varnothing \emptyset \\
\varnothing 715 \varnothing 58 \emptyset \\
\varnothing 7158 \varnothing 8 \emptyset \\
\varnothing 717465 \emptyset\end{array}$ & $\begin{array}{l}\text { Salt Fk Red R nr Vinson } \\
\text { Salt Fk Red R nr Wellington TX } \\
\text { Sand Ck Trib nr Kremlin } \\
\text { Sand Ck Trib nr Waynoka } \\
\text { Sand Ck at Bartlesville }\end{array}$ & $\begin{array}{l}\text { QWMP } \\
\text { QWMP } \\
\text { CS } \\
\text { CSR } \\
\text { QWP }\end{array}$ & $\begin{array}{l}\text { CNP } \\
\text { CNP } \\
\text { CP }\end{array}$ & & & $\begin{array}{r}1421 . \\
1222 . \\
7.21 \\
1.61\end{array}$ & $\begin{array}{l}1977 \\
1976-77 \\
1964-74 \\
1964-75 \\
1952-53\end{array}$ \\
\hline $\begin{array}{l}\emptyset 71746 \varnothing \emptyset \\
\emptyset 71746 \varnothing \emptyset \\
\emptyset 724188 \emptyset \\
\emptyset 7156548 \\
\varnothing 73236 \varnothing \emptyset\end{array}$ & $\begin{array}{l}\text { Sand Ck at Okesa } \\
\text { Sand } C k \text { at Okesa } \\
\text { Sand Ck nr Cromwell } \\
\text { Sand Ck nr Hawley } \\
\text { Sandstone Ck SWS } 22 \text { nr Cheyenne }\end{array}$ & $\begin{array}{l}\text { STR } \\
\text { QWP } \\
\text { CS } \\
\text { QWP } \\
\text { STR }\end{array}$ & $\begin{array}{l}\mathrm{CP} \\
\mathrm{CP}\end{array}$ & & $\begin{array}{l}\mathrm{C} \\
\mathrm{HG}\end{array}$ & $\begin{array}{l}139 . \\
139 . \\
9.48 \\
2.26\end{array}$ & $\begin{array}{l}1959- \\
1952-55,66-62 \\
1964-85 \\
1962 \\
1963-72\end{array}$ \\
\hline $\begin{array}{l}\emptyset 73216 \varnothing \emptyset \\
\emptyset 7321 \varnothing \varnothing \emptyset \\
\emptyset 732 \varnothing 5 \varnothing \emptyset \\
\emptyset 7322 \varnothing \varnothing \emptyset \\
\emptyset 7324 \varnothing \varnothing \emptyset\end{array}$ & $\begin{array}{l}\text { Sandstone Ck SWS } 3 \mathrm{nr} \text { Elk City } \\
\text { Sandstone Ck SWS } 5 \mathrm{nr} \text { Elk City } \\
\text { Sandstone Ck SWS } 6 \mathrm{nr} \text { Elk City } \\
\text { Sandstone Ck SWS } 9 \mathrm{nr} \text { Elk City } \\
\text { Sandstone Ck SWS } 1 \mathrm{nr} \text { Cheyenne }\end{array}$ & $\begin{array}{l}\text { STR } \\
\text { STR } \\
\text { STR } \\
\text { STR } \\
\text { STR }\end{array}$ & & & & $\begin{array}{l}0.62 \\
3.89 \\
6.46 \\
3.60 \\
6.33\end{array}$ & $\begin{array}{l}1953-74 \\
1953-74 \\
1953-74 \\
1952-74 \\
1952-74\end{array}$ \\
\hline $\begin{array}{l}\emptyset 7324 \varnothing \varnothing \emptyset \\
\varnothing 732 \varnothing \varnothing \varnothing \emptyset \\
\varnothing 73185 \varnothing \emptyset \\
\varnothing 7318 \varnothing \varnothing \emptyset \\
\varnothing 73176 \varnothing \emptyset\end{array}$ & $\begin{array}{l}\text { Sandstone CK SWS I nr Cheyenne } \\
\text { Sandstone CK SWS } 16 \mathrm{~A} \mathrm{nr} \text { EIk City } \\
\text { Sandstone CK SWS } 14 \mathrm{nr} \text { Cheyenne } \\
\text { Sandstone CK SWS } 16 \mathrm{nr} \text { Cheyenne } \\
\text { Sandstone CK SWS } 16 \mathrm{~A} \mathrm{nr} \text { Cheyenne }\end{array}$ & $\begin{array}{l}\text { QWP } \\
\text { STR } \\
\text { STR } \\
\text { STR } \\
\text { STR }\end{array}$ & $\mathrm{COP}$ & & & $\begin{array}{c}6.33 \\
2.87 \\
1.62 \\
11.5 \\
8.78\end{array}$ & $\begin{array}{l}1969-76 \\
1952-74 \\
1953-74 \\
1953-74 \\
1952-74\end{array}$ \\
\hline $\begin{array}{l}\emptyset 7319 \varnothing \varnothing \emptyset \\
\varnothing 7319 \varnothing \varnothing \emptyset \\
\varnothing 73195 \varnothing \emptyset \\
\varnothing 73196 \varnothing \emptyset \\
\varnothing 7323 \varnothing \varnothing \emptyset\end{array}$ & $\begin{array}{l}\text { Sandstone Ck SWS } 17 \mathrm{nr} \text { Cheyenne } \\
\text { Sandstone Ck SWS } 17 \mathrm{nr} \text { Cheyenne } \\
\text { Sandstone Ck } \mathrm{nr} \text { Berlin } \\
\text { Sandstone Ck } \mathrm{nr} \text { Berlin } \\
\text { Sandstone Ck nr Cheyenne }\end{array}$ & $\begin{array}{l}\text { STR } \\
\text { QWP } \\
\text { STR } \\
\text { QWP } \\
\text { STR }\end{array}$ & $\begin{array}{l}\mathrm{CP} \\
\mathrm{CP}\end{array}$ & & & $\begin{array}{l}10.1 \\
10.1 \\
44.9 \\
44.9 \\
87.1\end{array}$ & $\begin{array}{l}1953-74 \\
1969-7 \varnothing \\
1953-72 \\
1968 \\
1951-74\end{array}$ \\
\hline $\begin{array}{l}\emptyset 7323 \varnothing \varnothing \emptyset \\
\varnothing 71497 \varnothing \emptyset \\
\varnothing 729971 \emptyset \\
\varnothing 729971 \emptyset \\
\varnothing 729971 \emptyset\end{array}$ & $\begin{array}{l}\text { Sandstone Ck nr Cheyenne } \\
\text { Sandy Ck } n r \text { Byron } \\
\text { Sandy } C k n r \text { Eldorado } \\
\text { Sandy Ck } n r \text { Eldorado } \\
\text { Sandy } C k n r \text { Eldorado }\end{array}$ & $\begin{array}{l}\text { QWP } \\
\text { LF } \\
\text { STR } \\
\text { QWP } \\
\text { MISC }\end{array}$ & $\mathrm{CP}$ & & $J$ & $\begin{array}{l}87.1 \\
434 \\
280 \\
280 \\
280\end{array}$ & $\begin{array}{l}1951-62,57-58 \\
1965-73 \\
1960-64 \\
1952-55,58,61-63 \\
1987-\end{array}$ \\
\hline $\begin{array}{l}\emptyset 71496 \varnothing 8 \\
\varnothing 724606 \emptyset \\
\emptyset 724606 \emptyset \\
\emptyset 72457 \varnothing 3 \\
\emptyset 7335775\end{array}$ & $\begin{array}{l}\text { Sandy Ck nr Ingersoll } \\
\text { Sans Bois Ck nr Keota } \\
\text { Sans Bois Ck nr Keota } \\
\text { Sans Bois Ck nr Kinta } \\
\text { Sardis Lake at Clayton }\end{array}$ & $\begin{array}{l}\text { QWP } \\
\text { STR } \\
\text { QWP } \\
\text { QWP } \\
\text { RESP }\end{array}$ & $\begin{array}{l}\mathrm{CP} \\
\mathrm{CP} \\
\mathrm{CP}\end{array}$ & & C & $\begin{array}{l}346 . \\
346 . \\
276\end{array}$ & $\begin{array}{l}1962 \\
1938-42 \\
1958-63 \\
1961 \\
1983-\end{array}$ \\
\hline
\end{tabular}

* Continuous streamflow records for this period.

* Some records in intervening years.

*** Some records may have been collected previously. 
Table 1.--Alphabetical listing of current and historical gaging stations maintained by the U.S. Geological Survey

September 30, 1987--Continued

[Symbols defined on last page of report]

\begin{tabular}{|c|c|c|c|c|c|c|c|}
\hline $\begin{array}{l}\text { Station } \\
\text { Number }\end{array}$ & Station & $\begin{array}{l}\text { Type } \\
\text { of } \\
\text { Data }\end{array}$ & $\begin{array}{l}\text { Q.W. } \\
\text { Para- } \\
\text { meters }\end{array}$ & $\begin{array}{l}\text { Stage } \\
\text { Source }\end{array}$ & $\begin{array}{l}\text { Coop- } \\
\text { erator }\end{array}$ & $\begin{array}{c}\text { Drainage } \\
\text { Area } \\
(\mathrm{mile})\end{array}$ & Period of Record \\
\hline $\begin{array}{l}\emptyset 723385 \emptyset \\
\emptyset 73 \emptyset 148 \emptyset \\
\emptyset 7316891 \\
\emptyset 7315882 \\
\emptyset 715299 \emptyset\end{array}$ & $\begin{array}{l}\text { Sharp Ck Trib nr Turpin } \\
\text { Short Ck nr Sayre } \\
\text { Simon Ck at Pike } \\
\text { Simon Ck nr Oswalt } \\
\text { Skedee Ck at Pawnee Lake nr Pawnee }\end{array}$ & $\begin{array}{l}\text { CSR } \\
\text { CS } \\
\text { QWP } \\
\text { QWP } \\
\text { QWP }\end{array}$ & $\begin{array}{l}C P \\
C P \\
C P\end{array}$ & & $H G$ & $\begin{array}{l}1.00 \\
9.12\end{array}$ & $\begin{array}{l}1964-75 \\
1964-86 \\
1960-61 \\
1960-61 \\
1963-66\end{array}$ \\
\hline 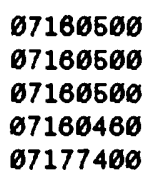 & $\begin{array}{l}\text { Skeleton Ck nr Lovell } \\
\text { Skeleton Ck } n r \text { Lovell } \\
\text { Skeleton Ck } n r \text { Lovell } \\
\text { Skeleton Ck nr Roxona } \\
\text { Skiatook Lake nr Skiatook }\end{array}$ & $\begin{array}{l}\text { STR } \\
\text { QWD } \\
\text { QWMP } \\
\text { QWP } \\
\text { RES }\end{array}$ & $\begin{array}{l}C P \\
C N P \\
C P\end{array}$ & $D C P$ & JG & $\begin{array}{l}410 . \\
410 \\
410 \\
364\end{array}$ & $\begin{array}{l}1949- \\
1951-65 \\
1975-79 \\
195 \varnothing \\
1984-\end{array}$ \\
\hline $\begin{array}{l}\emptyset 716565 \emptyset \\
\varnothing 7165559 \\
\varnothing 723256 \varnothing \\
\varnothing 71988 \emptyset \emptyset \\
\varnothing 71988 \emptyset \emptyset\end{array}$ & $\begin{array}{l}\text { Snake Ck nr Bixby } \\
\text { Snake Ck nr Leonard } \\
\text { South Fk Trib nr Guymon } \\
\text { South Fk nr Porum } \\
\text { South Fk nr Porum }\end{array}$ & $\begin{array}{l}\text { CS } \\
\text { QWP } \\
\text { CS } \\
\text { QWMP } \\
\text { PR }\end{array}$ & $\begin{array}{l}C P \\
S \\
C P\end{array}$ & & $C$ & $\begin{array}{l}50.0 \\
\\
0.26 \\
48.7 \\
48.7\end{array}$ & $\begin{array}{l}1961-70 * * 71-76 \\
1960-61 \\
1975- \\
1980-83 \\
1980-83\end{array}$ \\
\hline $\begin{array}{l}\emptyset 71988 \varnothing \emptyset \\
67191223 \\
671912 \varnothing \varnothing \\
\varnothing 71912 \varnothing \emptyset \\
\varnothing 7191366\end{array}$ & $\begin{array}{l}\text { South Fk } n r \text { Porum } \\
\text { Spavinaw } C k n r \text { Jay } \\
\text { Spavinaw } C k n r \text { Row } \\
\text { Spavinaw } C k n r \text { Row } \\
\text { Spavinaw Ck nr Spavinaw }\end{array}$ & $\begin{array}{l}\text { SED } \\
\text { QWP } \\
\text { STR } \\
\text { QWP } \\
\text { QWP }\end{array}$ & $\begin{array}{l}S \\
C P \\
C P \\
C P\end{array}$ & & & $\begin{array}{l}48.7 \\
128 . \\
128\end{array}$ & $\begin{array}{l}1980-83 \\
1958-59,61 \\
1959-62 \\
1959-61 \\
1948-61\end{array}$ \\
\hline $\begin{array}{l}\emptyset 719122 \varnothing \\
\emptyset 719122 \emptyset \\
\emptyset 719122 \varnothing \\
\emptyset 7327437 \\
\varnothing 71596 \varnothing \emptyset\end{array}$ & $\begin{array}{l}\text { Spavinaw Ck nr Sycamore } \\
\text { Spavinaw Ck nr Sycamore } \\
\text { Spavinaw Ck nr Sycamore } \\
\text { Spring Ck Trib nr Middleberg } \\
\text { Spring Ck at Lansbrook Lane, OKC }\end{array}$ & $\begin{array}{l}\text { STR } \\
\text { QWMP } \\
\text { QWP } \\
\text { QWP } \\
\text { CSR }\end{array}$ & $\begin{array}{l}\text { CNP } \\
\text { CNP } \\
C\end{array}$ & & $\begin{array}{l}P G \\
A\end{array}$ & $\begin{array}{l}133 . \\
133 . \\
133 . \\
\quad 0.76 \\
3.17\end{array}$ & $\begin{array}{l}1961- \\
1977 \\
1968,77,86- \\
1969-71 \\
1976-78\end{array}$ \\
\hline $\begin{array}{l}\emptyset 715961 \emptyset \\
\emptyset 724227 \emptyset \\
\emptyset 7229426 \\
\emptyset 7327432 \\
\varnothing 7325763\end{array}$ & $\begin{array}{l}\text { Spring } C k \text { at } N \text { MacArthur Blvd, OKC } \\
\text { Spring } C k \text { at Pine Oak Dr, Edmond } \\
\text { Spring } C k n r \text { Ada } \\
\text { Spring } C k n r \text { Blanchard } \\
\text { Spring } C k n r \text { Eakly }\end{array}$ & $\begin{array}{l}\text { CSR } \\
\text { CSR } \\
\text { QWP } \\
\text { QWP } \\
\text { QWP }\end{array}$ & $\begin{array}{l}C P \\
C \\
C P\end{array}$ & & & $\begin{array}{l}8.43 \\
1.32 \\
1.19\end{array}$ & $\begin{array}{l}1976-78 \\
1976-81 \\
1952-53 \\
1968-71 \\
1961\end{array}$ \\
\hline $\begin{array}{l}\emptyset 73 ø 1486 \\
\varnothing 7327 ø 31 \\
\emptyset 73 \varnothing 4299 \\
\emptyset 7327436 \\
\emptyset 7188 \varnothing \emptyset \emptyset\end{array}$ & $\begin{array}{l}\text { Spring Ck nr Elk City } \\
\text { Spring Ck nr Gracemont } \\
\text { Spring Ck nr Sentinel } \\
\text { Spring Ck nr Tabler } \\
\text { Spring } R \text { nr Quapaw }\end{array}$ & $\begin{array}{l}\text { CS } \\
\text { QWP } \\
\text { QWP } \\
\text { QWP } \\
\text { STR }\end{array}$ & $\begin{array}{l}C P \\
C P \\
C\end{array}$ & $D C P$ & LGC & $\begin{array}{l}6.93 \\
2.28 \\
2610 .\end{array}$ & $\begin{array}{l}1968-72 \\
1961 \\
1960 \\
1968-71 \\
1939-\end{array}$ \\
\hline $\begin{array}{l}\emptyset 7188 \emptyset \emptyset \emptyset \\
\emptyset 7188 \emptyset \emptyset \emptyset \\
\emptyset 73 \varnothing 1462 \\
\emptyset 724218 \emptyset \\
\emptyset 7162 \emptyset \emptyset \emptyset\end{array}$ & $\begin{array}{l}\text { Spring } R \text { nr Quapaw } \\
\text { Spring } R \text { nr Quapaw } \\
\text { Starvation Ck nr Prentiss } \\
\text { Stidham Ck Trib nr Dustin } \\
\text { Stillwater Ck at Stillwater }\end{array}$ & $\begin{array}{l}\text { QWP } \\
\text { QWMP } \\
\text { LF } \\
\text { CS } \\
\text { STR }\end{array}$ & $\begin{array}{l}C P \\
C N P\end{array}$ & & & $\begin{array}{l}2510 . \\
2510 . \\
44.6 \\
2.56 \\
168 .\end{array}$ & $\begin{array}{l}1948-68,60-63 \\
1973-79 \\
1964-73 \\
1964-76 \\
1935-38\end{array}$ \\
\hline
\end{tabular}

* Continuous streamflow records for this period.

** Some records in intervening years.

*** Some records may have been collected previously. 
Table 1.--Alphabetical listing of current and historical gaging stations maintained by the U.S. Geological Survey

September 30, 1987--Continued

[Symbols defined on last page of report]

\begin{tabular}{|c|c|c|c|c|c|c|c|}
\hline $\begin{array}{r}\text { Station } \\
\text { Number }\end{array}$ & Station & $\begin{array}{l}\text { Type } \\
\text { of } \\
\text { Data }\end{array}$ & $\begin{array}{l}\text { Q.W. } \\
\text { Para- } \\
\text { meters }\end{array}$ & $\begin{array}{l}\text { Stage } \\
\text { Source }\end{array}$ & $\begin{array}{l}\text { Coop- } \\
\text { erator }\end{array}$ & $\begin{array}{c}\text { Drainage } \\
\text { Area } \\
(\mathrm{mile})\end{array}$ & Period of Record \\
\hline $\begin{array}{l}\emptyset 7162 \emptyset \emptyset \emptyset \\
\emptyset 73254 \emptyset \emptyset \\
\emptyset 73254 \emptyset \emptyset \\
\emptyset 7327 \emptyset \emptyset \emptyset \\
\emptyset 7327 \varnothing 0 \emptyset\end{array}$ & $\begin{array}{l}\text { Stillwater Ck at Stillwater } \\
\text { Stinking Ck nr Carnegie } \\
\text { Stinking Ck nr Carnegie } \\
\text { Sugar Ck } n r \text { Gracemont } \\
\text { Sugar Ck nr Gracemont }\end{array}$ & $\begin{array}{l}\text { SED } \\
\text { LF } \\
\text { QWP } \\
\text { STRP } \\
\text { QWP }\end{array}$ & $\begin{array}{l}\mathrm{S} \\
\mathrm{CP} \\
\mathrm{CP}\end{array}$ & & & $\begin{array}{l}168 \\
104 \\
104 \\
208 \\
208\end{array}$ & $\begin{array}{l}1934-37 \\
1951-66,58-61 \\
1962-65,68 \\
1956-74 \\
1956-6 \varnothing\end{array}$ \\
\hline $\begin{array}{l}\emptyset 72487 \emptyset \emptyset \\
\emptyset 72487 \emptyset \emptyset \\
\emptyset 72487 \emptyset \emptyset \\
\emptyset 73 \emptyset 831 \emptyset \\
\emptyset 717149 \emptyset\end{array}$ & $\begin{array}{l}\text { Sugarloaf Ck } n r \text { Monroe } \\
\text { Sugarloaf Ck } n r \text { Monroe } \\
\text { Sugarloaf Ck } n r \text { Monroe } \\
\text { Suttle Ck } n r \text { Davidson } \\
\text { Sweetwater Ck nr Claremore }\end{array}$ & $\begin{array}{l}\text { STR } \\
\text { QWP } \\
\text { SED } \\
\text { LF } \\
\text { QWMP }\end{array}$ & $\begin{array}{l}\text { CNP } \\
S \\
C P\end{array}$ & & & $\begin{array}{l}53.6 \\
53.6 \\
53.6 \\
65.0\end{array}$ & $\begin{array}{l}1978-81 \\
1978-81 \\
1978-81 \\
1965-66 \\
1980-83\end{array}$ \\
\hline $\begin{array}{l}\emptyset 7171490 \\
\emptyset 717149 \emptyset \\
\emptyset 73 \emptyset 142 \emptyset \\
\emptyset 73 \emptyset 1425 \\
\emptyset 719651 \emptyset\end{array}$ & $\begin{array}{l}\text { Sweetwater Ck nr Claremore } \\
\text { Sweetwater Ck nr Claremore } \\
\text { Sweetwater Ck nr Sweetwater } \\
\text { Sweetwater Ck nr Texas Line } \\
\text { Tahlequah Ck at Tahlequah }\end{array}$ & $\begin{array}{l}\text { PR } \\
\text { SED } \\
\text { STR } \\
\text { QWP } \\
\text { QWMP }\end{array}$ & $\begin{array}{l}S \\
C P \\
\text { CNP }\end{array}$ & & $J G$ & 13.4 & $\begin{array}{l}1980-83 \\
1980-83 \\
1986- \\
1953-54 \\
1976-77\end{array}$ \\
\hline $\begin{array}{l}\emptyset 7245 \emptyset 25 \\
\emptyset 7246 \varnothing 25 \\
\emptyset 7245 \varnothing 25 \\
\emptyset 7245 \emptyset 2 \emptyset \\
\emptyset 7246 \emptyset 2 \emptyset\end{array}$ & $\begin{array}{l}\text { Taloka Ck Trib nr Stigler } \\
\text { Taloka Ck Trib nr Stigler } \\
\text { Taloka Ck Trib nr Stigler } \\
\text { Taloka Ck at Stigler } \\
\text { Taloka Ck at Stigler }\end{array}$ & $\begin{array}{l}\text { PR } \\
\text { QWP } \\
\text { SED } \\
\text { PR } \\
\text { QWP }\end{array}$ & $\begin{array}{l}\mathrm{CN} \\
\mathrm{P} \\
\mathrm{S} \\
\mathrm{CNP}\end{array}$ & & & $\begin{array}{l}2.04 \\
2.04 \\
2.04 \\
3.98 \\
3.98\end{array}$ & $\begin{array}{l}1979-81 \\
1978-81 \\
1979-81 \\
1978-81 \\
1978-81\end{array}$ \\
\hline $\begin{array}{l}\emptyset 724502 \emptyset \\
\emptyset 7245 \emptyset 3 \emptyset \\
\emptyset 7245 \emptyset 3 \emptyset \\
\emptyset 7246 \varnothing 3 \emptyset \\
\emptyset 7185 \varnothing 95\end{array}$ & $\begin{array}{l}\text { Taloka Ck at Stigler } \\
\text { Taloka Ck nr Stigler } \\
\text { Taloka Ck nr Stigler } \\
\text { Taloka Ck nr Stigler } \\
\text { Tar Ck at 22nd St at Miami }\end{array}$ & $\begin{array}{l}\text { SED } \\
\text { STR } \\
\text { QWP } \\
\text { SED } \\
\text { STR }\end{array}$ & $\begin{array}{l}\mathrm{S} \\
\mathrm{CNP} \\
\mathrm{S}\end{array}$ & & JG & $\begin{array}{l}3.98 \\
20.1 \\
20.1 \\
20.1 \\
44.7\end{array}$ & $\begin{array}{l}1978-81 \\
1978-81 \\
1978-81 \\
1978-81 \\
1984-\end{array}$ \\
\hline $\begin{array}{l}\emptyset 7185 \emptyset 95 \\
\emptyset 71851 \emptyset \emptyset \\
\emptyset 71975 \varnothing \emptyset \\
\emptyset 7336 \emptyset \emptyset \emptyset \\
\emptyset 7336 \emptyset \emptyset \emptyset\end{array}$ & $\begin{array}{l}\text { Tar Ck at 22nd St at Miami } \\
\text { Tar Ck at Miami } \\
\text { Tenkiller Ferry Lake nr Gore } \\
\text { Tenmile Ck nr Miller } \\
\text { Tenmile Ck nr Miller }\end{array}$ & $\begin{array}{l}\text { QWP } \\
\text { STR } \\
\text { RES } \\
\text { QWP } \\
\text { CS }\end{array}$ & $\begin{array}{l}\text { CNOP } \\
\mathrm{S} \\
\mathrm{C}\end{array}$ & $D C P$ & $\begin{array}{l}J \\
J G \\
C\end{array}$ & $\begin{array}{r}44.7 \\
52.0 \\
1610 . \\
68.0 \\
68.0\end{array}$ & $\begin{array}{l}1985- \\
1980-84 \\
1952- \\
1956-58 \\
1957-70,71-86\end{array}$ \\
\hline $\begin{array}{l}\emptyset 7232450 \\
6715465 \emptyset \\
\emptyset 7231962 \\
\emptyset 7231962 \\
\emptyset 7231966\end{array}$ & $\begin{array}{l}\text { Tepee Ck nr Eva } \\
\text { Tesesquite Ck nr Kenton } \\
\text { Ti Ck abv unnamed Trib nr Blanco } \\
\mathrm{Ti} \text { Ck abv unnamed Trib nr Blanco } \\
\text { Ti Ck nr Blanco }\end{array}$ & $\begin{array}{l}\text { LF } \\
\text { CS } \\
\text { CS } \\
\text { QWP } \\
\text { STR }\end{array}$ & CNOP & & HG & $\begin{array}{l}95.0 \\
25.4 \\
1.83 \\
1.83 \\
4.82\end{array}$ & $\begin{array}{l}1967-73 \\
1964-86 \\
1980-81 \\
1980-81 \\
1980-81\end{array}$ \\
\hline $\begin{array}{l}\emptyset 7231966 \\
\emptyset 7231965 \\
\emptyset 73 \emptyset 54 \emptyset \emptyset \\
\emptyset 732672 \emptyset \\
\emptyset 7158650\end{array}$ & $\begin{array}{l}\text { Ti Ck nr Blanco } \\
\text { Ti Ck nr Blanco } \\
\text { Tom Steod Res nr Mountain Park } \\
\text { Tonkawa Ck nr Anadarko } \\
\text { Turkey Ck Trib nr Goltry }\end{array}$ & $\begin{array}{l}\text { QWP } \\
\text { SED } \\
\text { RES } \\
\text { QWD } \\
\text { CSR }\end{array}$ & $\begin{array}{l}\text { BCNP } \\
\mathrm{S} \\
\mathrm{CP}\end{array}$ & & $\begin{array}{l}\mathrm{C} \\
\mathrm{HG}\end{array}$ & $\begin{array}{r}4.82 \\
4.82 \\
26.0 \\
6.68\end{array}$ & $\begin{array}{l}1980-81 \\
1980-81 \\
1986- \\
1968-71 \\
1964-81\end{array}$ \\
\hline
\end{tabular}

* Continuous streamflow records for this period.

** Some records in intervening years.

*** Some records may have been collected previously. 
Table 1.--Alphabetical listing of current and historical gaging stations maintained by the U.S. Geological Survey

September 90, 1987--Continued

[Symbols defined on last page of report]

\begin{tabular}{|c|c|c|c|c|c|c|c|}
\hline $\begin{array}{l}\text { Station } \\
\text { Number }\end{array}$ & Station & $\begin{array}{l}\text { Typo } \\
\text { of } \\
\text { Data }\end{array}$ & $\begin{array}{l}\text { Q.W. } \\
\text { Para- } \\
\text { meters }\end{array}$ & $\begin{array}{l}\text { Stage } \\
\text { Source }\end{array}$ & $\begin{array}{l}\text { Coop- } \\
\text { orator }\end{array}$ & $\begin{array}{c}\text { Drainage } \\
\text { Area } \\
(m i l e)\end{array}$ & Period of Record \\
\hline $\begin{array}{l}\emptyset 716855 \varnothing \\
\varnothing 73 \varnothing 11 \varnothing \emptyset \\
\varnothing 73 \varnothing 11 \varnothing \emptyset \\
\varnothing 73 \varnothing 11 \varnothing \emptyset \\
\varnothing 73 \varnothing 11 \varnothing \emptyset\end{array}$ & 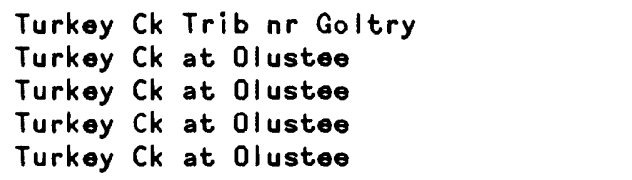 & $\begin{array}{l}\text { CS } \\
\text { STR } \\
\text { QWP } \\
\text { MISC } \\
\text { STR }\end{array}$ & $\mathrm{CP}$ & & $\begin{array}{l}H G \\
J G\end{array}$ & $\begin{array}{l}5 . \varnothing 8 \\
293 . \\
293 . \\
293 \\
293\end{array}$ & $\begin{array}{l}1964-85 \\
1960-64 \\
1952,54-67,61-63 \\
1987- \\
1987-\end{array}$ \\
\hline $\begin{array}{l}\emptyset 7148379 \\
\varnothing 722822 \emptyset \\
\varnothing 71592 \emptyset 3 \\
\varnothing 7159 \varnothing \emptyset \emptyset \\
\varnothing 7159 \varnothing \emptyset \emptyset\end{array}$ & $\begin{array}{l}\text { Turkey Ck nr Alva } \\
\text { Turkey Ck nr Camargo } \\
\text { Turkey Ck nr Dover } \\
\text { Turkey Ck nr Drummond } \\
\text { Turkey Ck nr Drummond }\end{array}$ & $\begin{array}{l}\text { QWP } \\
\text { LF } \\
\text { QWP } \\
\text { CS } \\
\text { QWP }\end{array}$ & $\begin{array}{l}\mathrm{CNP} \\
\mathrm{CP} \\
\mathrm{CP}\end{array}$ & & & $\begin{array}{l}86 . \emptyset \\
248 . \\
248 .\end{array}$ & $\begin{array}{l}1962 \\
1965-73 \\
1962-54,62 \\
1948-7 \emptyset, 71-74 \\
1948,52-59\end{array}$ \\
\hline 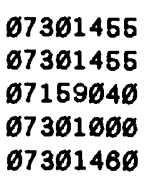 & $\begin{array}{l}\text { Turkey Ck nr Erick } \\
\text { Turkey Ck nr Erick } \\
\text { Turkey Ck nr Hennessey } \\
\text { Turkey Ck nr Olustee } \\
\text { Turkey Ck nr Sayre }\end{array}$ & $\begin{array}{l}\text { CS } \\
\text { QWP } \\
\text { QWP } \\
\text { STR } \\
\text { LF }\end{array}$ & $\begin{array}{l}C P \\
C P\end{array}$ & & HG & $\begin{array}{r}19.8 \\
19.8 \\
244 . \\
47.5\end{array}$ & $\begin{array}{l}1964-85 \\
1953 \\
1951 \\
1995-\varnothing 8 \\
1953-56,65-73\end{array}$ \\
\hline $\begin{array}{l}\emptyset 73 \emptyset 146 \emptyset \\
\emptyset 724121 \emptyset \\
\emptyset 7329851 \\
\emptyset 717845 \emptyset \\
\emptyset 71785 \varnothing \emptyset\end{array}$ & $\begin{array}{l}\text { Turkey Ck nr Sayre } \\
\text { Twin Ck at SW29, OKC } \\
\text { Vendome Well Outflow at Sulphur } \\
\text { Verdigris R at Catoosa } \\
\text { Verdigris R nr Catoosa }\end{array}$ & $\begin{array}{l}\text { QWP } \\
\text { CSR } \\
\text { STR } \\
\text { STGU } \\
\text { STR }\end{array}$ & $\mathrm{CP}$ & DCP & $\begin{array}{l}b \\
c\end{array}$ & $\begin{array}{r}47.5 \\
3.35 \\
7789 .\end{array}$ & $\begin{array}{l}1953 \\
1976-81 \\
1986- \\
1903-\varnothing 5\end{array}$ \\
\hline 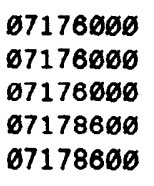 & $\begin{array}{l}\text { Verdigris } R n r \text { Claremore } \\
\text { Verdigris } R n r \text { Claremore } \\
\text { Verdigris } R n r \text { Claremore } \\
\text { Verdigris } R \text { nr Inola } \\
\text { Verdigris R nr Inola }\end{array}$ & $\begin{array}{l}\text { STR } \\
\text { QWD } \\
\text { QWMP } \\
\text { STRP } \\
\text { QWP }\end{array}$ & $\begin{array}{l}\text { CP } \\
\text { CNP } \\
\text { BCNP }\end{array}$ & DCP & C & $\begin{array}{l}6534 \\
6534 \\
6534 \\
7911 \\
7911\end{array}$ & $\begin{array}{l}1935- \\
1948-54,59 \\
1978-79 \\
1946-7 \varnothing \\
1948-72\end{array}$ \\
\hline 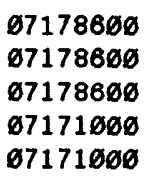 & $\begin{array}{l}\text { Verdigris R } n r \text { Inola } \\
\text { Verdigris } R n r \text { Inola } \\
\text { Verdigris } R n r \text { Inola } \\
\text { Verdigris } R n r \text { Lenapah } \\
\text { Verdigris } R \text { nr Lenapah }\end{array}$ & $\begin{array}{l}\text { QWMP } \\
\text { SED } \\
\text { STGU } \\
\text { STR } \\
\text { QWP }\end{array}$ & $\begin{array}{l}\text { CNP } \\
\mathrm{S}\end{array}$ & $\begin{array}{l}D C P \\
D C P\end{array}$ & $\begin{array}{l}c \\
C\end{array}$ & $\begin{array}{l}7911 . \\
7911 . \\
7911 . \\
3639 . \\
3639 .\end{array}$ & $\begin{array}{l}1977 \\
1948-72 \\
1986- \\
1938- \\
1945,52-64\end{array}$ \\
\hline 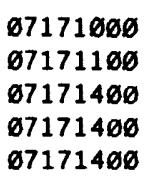 & $\begin{array}{l}\text { Verdigris } R \text { nr Lenapah } \\
\text { Verdigris } R n r \text { Nowata } \\
\text { Verdigris } R n r \text { Oologah } \\
\text { Verdigris } R n r \text { Oologah } \\
\text { Verdigris } R n r \text { Oologah }\end{array}$ & $\begin{array}{l}\text { QWMP } \\
\text { QWP } \\
\text { STR } \\
\text { QWP } \\
\text { QWMP }\end{array}$ & $\begin{array}{l}\text { CNP } \\
\text { CP } \\
\text { CP } \\
\text { CNP }\end{array}$ & & $\mathrm{C}$ & $\begin{array}{l}3639 . \\
4339 . \\
4339 \\
4339\end{array}$ & $\begin{array}{l}1973-79 \\
1952-63 \\
1961- \\
1962-63,65-75 \\
1973-79\end{array}$ \\
\hline $\begin{array}{l}\emptyset 71716 \varnothing \emptyset \\
\varnothing 717 \varnothing 95 \emptyset \\
\varnothing 717126 \varnothing \\
\varnothing 71714 \varnothing 5 \\
\varnothing 7245 \varnothing 9 \emptyset\end{array}$ & $\begin{array}{l}\text { Verdigris } R \text { nr Sageeyah } \\
\text { Verdigris } R \text { nr South Coffeyville } \\
\text { Verdigris } R n r \text { Talala } \\
\text { Verdigris R, Hwy } 88 \mathrm{Br}, n r \text { Claremore } \\
\text { Vian Ck nr Vian }\end{array}$ & $\begin{array}{l}\text { STRP } \\
\text { QWP } \\
\text { QWP } \\
\text { QWD } \\
\text { CS }\end{array}$ & $\begin{array}{l}C P \\
C P \\
C P\end{array}$ & & & $\begin{array}{l}44 ø 2 . \\
4339 .\end{array}$ & $\begin{array}{l}1939-45 \\
1952-53 \\
1952-53 \\
1952-53,59 \\
1966-72\end{array}$ \\
\hline
\end{tabular}

* Continuous streamflow records for this period.

** Some records in intervening years.

*** Some records may have been collected previously. 
Table 1.--Alphabetical listing of current and historical gaging stations maintained by the U.S. Geological Survey

September so, 1987--Continued

[Symbols defined on last page of report]

\begin{tabular}{|c|c|c|c|c|c|c|c|}
\hline $\begin{array}{r}\text { Station } \\
\text { Number }\end{array}$ & Station & $\begin{array}{c}\text { Type } \\
\text { of } \\
\text { Data }\end{array}$ & $\begin{array}{l}\text { Q.W. } \\
\text { Para- } \\
\text { meters }\end{array}$ & $\begin{array}{l}\text { Stage } \\
\text { Source }\end{array}$ & $\begin{array}{l}\text { Coop- } \\
\text { orator }\end{array}$ & $\begin{array}{c}\text { Drainage } \\
\text { Area } \\
(m i l e)\end{array}$ & Period of Record \\
\hline $\begin{array}{l}\emptyset 7246 \varnothing 9 \emptyset \\
\varnothing 716 \varnothing 55 \emptyset \\
\varnothing 716 \varnothing 55 \emptyset \\
\emptyset 732742 \emptyset \\
\varnothing 731124 \emptyset\end{array}$ & $\begin{array}{l}\text { Vian Ck nr Vian } \\
\text { W Beaver Ck nr Orlando } \\
\text { W Beaver Ck nr Orlando } \\
\text { W Bitter Ck nr Tabler } \\
\text { W Cache Ck nr Cookietown }\end{array}$ & $\begin{array}{l}\text { QWP } \\
\text { CS } \\
\text { CSR } \\
\text { QWD } \\
\text { LF }\end{array}$ & $\mathrm{CP}$ & & $H G$ & $\begin{array}{r}19.6 \\
13.9 \\
13.9 \\
60.8 \\
1112 .\end{array}$ & $\begin{array}{l}1958-59 \\
1964-85 \\
1964-76 \\
* * 1953,61,65-71 \\
1951-55,65-73\end{array}$ \\
\hline $\begin{array}{l}\emptyset 731124 \emptyset \\
\varnothing 724671 \emptyset \\
\varnothing 72467 \varnothing \emptyset \\
\varnothing 719 \varnothing 62 \emptyset \\
\varnothing 719 \varnothing 62 \varnothing\end{array}$ & $\begin{array}{l}\text { W Cache CK nr Cookietown } \\
\text { W D Mayo L D nr Braden DS } \\
\text { W D Mayo L D nr Braden US } \\
\text { W Fk Big Cabin Ck nr Centralia } \\
\text { W Fk Big Cabin Ck nr Centralia }\end{array}$ & $\begin{array}{l}\text { QWP } \\
\text { RESU } \\
\text { RESU } \\
\text { QWMP } \\
\text { PR }\end{array}$ & $\begin{array}{l}\mathrm{CP} \\
\mathrm{CP}\end{array}$ & DCP & $\begin{array}{l}c \\
C\end{array}$ & $\begin{array}{l}13.1 \\
13.1\end{array}$ & $\begin{array}{l}1952-65,6 \emptyset-63 \\
1986- \\
1986- \\
1980-83 \\
1980-83\end{array}$ \\
\hline $\begin{array}{l}\emptyset 719 \varnothing 62 \emptyset \\
\varnothing 71625 \varnothing \emptyset \\
\varnothing 71625 \varnothing \emptyset \\
\emptyset 7315672 \\
\varnothing 7316681\end{array}$ & $\begin{array}{l}\text { W Fk Big Cabin Ck nr Centralia } \\
\text { W Fk Brush Ck nr Stillwater } \\
\text { W Fk Brush Ck nr Stillwater } \\
\text { W Mud Ck nr Atlee } \\
\text { W Mud Ck nr Ringling }\end{array}$ & $\begin{array}{l}\text { SED } \\
\text { STR } \\
\text { SED } \\
\text { QWP } \\
\text { QWP }\end{array}$ & $\begin{array}{l}S \\
\mathrm{~S} \\
\mathrm{CP}\end{array}$ & & & $\begin{array}{l}13.1 \\
13.1 \\
13.1\end{array}$ & $\begin{array}{l}1980-83 \\
1935-38 \\
1934-37 \\
1961 \\
196 \emptyset\end{array}$ \\
\hline $\begin{array}{l}\emptyset 7315681 \\
\varnothing 73 \varnothing 5198 \\
\varnothing 73 \varnothing 55 \varnothing \emptyset \\
\varnothing 73 \varnothing 55 \varnothing \emptyset \\
\emptyset 732732 \emptyset\end{array}$ & $\begin{array}{l}\text { w Mud Ck nr Ringling } \\
\text { W Otter Ck at Cold Spring } \\
\text { w Otter Ck at Snyder Lake nr Mt Park } \\
\text { W Otter Ck at Snyder Lake nr Mt Park } \\
\text { W Salt Ck nr Chickasha }\end{array}$ & $\begin{array}{l}\text { QWP } \\
\text { QWP } \\
\text { STR } \\
\text { QWP } \\
\text { QWD }\end{array}$ & $\begin{array}{l}\mathrm{CP} \\
\mathrm{CP} \\
\mathrm{CP} \\
\mathrm{COP}\end{array}$ & & DG & $\begin{array}{l}132 . \\
132 . \\
22.0\end{array}$ & $\begin{array}{l}1963 \\
1961 \\
1963-68,61- \\
196 \varnothing \\
1968-71\end{array}$ \\
\hline $\begin{array}{l}\varnothing 7313211 \\
\varnothing 73159 \varnothing \emptyset \\
\varnothing 73159 \varnothing \varnothing \\
\varnothing 73159 \emptyset \emptyset \\
\varnothing 7315873\end{array}$ & $\begin{array}{l}\text { Walker Ck nr Corum } \\
\text { Walnut Bayou nr Burneyville } \\
\text { Walnut Bayou nr Burneyville } \\
\text { Walnut Bayou nr Burneyville } \\
\text { Walnut Bayou nr Oswalt }\end{array}$ & $\begin{array}{l}\text { QWP } \\
\text { STR } \\
\text { QWD } \\
\text { QWMP } \\
\text { QWP }\end{array}$ & $\begin{array}{l}\mathrm{CP} \\
\mathrm{CP} \\
\mathrm{CNP} \\
\mathrm{CP}\end{array}$ & & & $\begin{array}{l}314 . \\
314 . \\
314 .\end{array}$ & $\begin{array}{l}1961 \\
1961-64,69-71 \\
1960-62,69-71 \\
1975-79 \\
1960\end{array}$ \\
\hline $\begin{array}{l}\emptyset 72293 \varnothing \emptyset \\
\varnothing 72293 \varnothing \emptyset \\
\emptyset 72293 \varnothing \emptyset \\
\emptyset 722922 \emptyset \\
\varnothing 71478 \varnothing \emptyset\end{array}$ & $\begin{array}{l}\text { Walnut } C_{k} \text { at Purcell } \\
\text { Walnut } C k \text { at Purcell } \\
\text { Walnut } C k \text { at Purcell } \\
\text { Walnut } C k \text { nr Blanchard } \\
\text { Walnut } R \text { at Winfield KS }\end{array}$ & $\begin{array}{l}\text { STR } \\
\text { QWP } \\
\text { QWMP } \\
\text { CS } \\
\text { QWP }\end{array}$ & $\begin{array}{l}\mathrm{CP} \\
\mathrm{CNP} \\
\mathrm{C}\end{array}$ & & JG & $\begin{array}{l}202 . \\
202 \\
202 \\
1.26 \\
1872\end{array}$ & $\begin{array}{l}1965- \\
1950-55,58-62 \\
1976-77 \\
1964-72 \\
196 \varnothing-61\end{array}$ \\
\hline $\begin{array}{l}\emptyset 731641 \emptyset \\
\varnothing 73281 \varnothing \emptyset \\
\varnothing 73281 \varnothing \emptyset \\
\emptyset 73265 \varnothing \emptyset \\
\varnothing 73265 \varnothing \emptyset\end{array}$ & $\begin{array}{l}\text { Washita } R \text { Trib nr Crawford } \\
\text { Washita } R \text { at Alex } \\
\text { Washita } R \text { at Alex } \\
\text { Washita } R \text { at Anadarko } \\
\text { Washita } R \text { at Anadarko }\end{array}$ & $\begin{array}{l}\text { CS } \\
\text { STRP } \\
\text { QWD } \\
\text { STRP } \\
\text { QWD }\end{array}$ & $\begin{array}{l}\mathrm{CP} \\
\mathrm{CP}\end{array}$ & OBS & $\begin{array}{l}J G \\
J G\end{array}$ & $\begin{array}{l}2.18 \\
4787 \\
4787 \\
3656 \\
3656\end{array}$ & $\begin{array}{l}1965-72 \\
1964-86 \\
1965-71 \\
* * 1902-68,63- \\
1952,65-71\end{array}$ \\
\hline $\begin{array}{l}\emptyset 73265 \varnothing \emptyset \\
\varnothing 73265 \varnothing \emptyset \\
\varnothing 73265 \varnothing \emptyset \\
\emptyset 73255 \varnothing \emptyset \\
\emptyset 733 \varnothing \varnothing \varnothing \emptyset\end{array}$ & $\begin{array}{l}\text { Washita } R \text { at Anadarko } \\
\text { Washita } R \text { at Carnegie } \\
\text { Washita } R \text { at Carnegie } \\
\text { Washita } R \text { at Carnegie } \\
\text { Washita } R \text { nr Berwyn }\end{array}$ & $\begin{array}{l}\text { QWMP } \\
\text { STR } \\
\text { QWP } \\
\text { QWMP } \\
\text { STR }\end{array}$ & $\begin{array}{l}\text { CNP } \\
\text { CNP } \\
\text { CNP }\end{array}$ & $\begin{array}{l}\text { OBS } \\
\text { OBS } \\
\text { OBS }\end{array}$ & $\begin{array}{l}J G \\
V G\end{array}$ & $\begin{array}{l}3656 \\
3129 . \\
3129 . \\
3129 \\
6815\end{array}$ & $\begin{array}{l}1976-79 \\
1937- \\
1948-87 \\
1973-76 \\
1924-26\end{array}$ \\
\hline
\end{tabular}

* Continuous streamflow records for this period.

** Some records in intervening years.

*** Some records may have been collected previously. 
Table 1.--Alphabetical listing of current and historical gaging stations maintained by the U.S. Geological Survey

September 90 , 1987--Continued

[Symbols defined on last page of report]

\begin{tabular}{|c|c|c|c|c|c|c|c|c|}
\hline $\begin{array}{l}\text { Station } \\
\text { Number }\end{array}$ & Station & Name & $\begin{array}{l}\text { Type } \\
\text { of } \\
\text { Data }\end{array}$ & $\begin{array}{l}\text { Q.W. } \\
\text { Para- } \\
\text { meters }\end{array}$ & $\begin{array}{l}\text { Stage } \\
\text { Source }\end{array}$ & $\begin{array}{l}\text { Coop- } \\
\text { erator }\end{array}$ & $\begin{array}{c}\text { Drainage } \\
\text { Area } \\
(m i l e)\end{array}$ & Period of Record \\
\hline 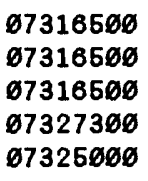 & $\begin{array}{l}\text { Washita } R n r \\
\text { Washita } R n r \\
\text { Washita } R n r \\
\text { Washita } R n r \\
\text { Washita } R n r\end{array}$ & $\begin{array}{l}\text { Cheyenne } \\
\text { Cheyenne } \\
\text { Cheyenne } \\
\text { Chickasha } \\
\text { Clinton }\end{array}$ & $\begin{array}{l}\text { STR } \\
\text { QWP } \\
\text { QWMP } \\
\text { QWP } \\
\text { STR }\end{array}$ & $\begin{array}{l}\text { CNP } \\
\text { CNP } \\
\text { CP }\end{array}$ & OBS & $J G$ & $\begin{array}{l}794 . \\
794 . \\
794 . \\
1977 .\end{array}$ & $\begin{array}{l}1937- \\
1950-63,60-61,69-73 \\
1973-75 \\
1952-53,55,58-61 \\
1935-\end{array}$ \\
\hline 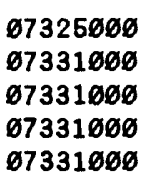 & $\begin{array}{l}\text { Washita } R n r \\
\text { Washita } R n r \\
\text { Washita } R n r \\
\text { Washita } R n r \\
\text { Washita } R n r\end{array}$ & $\begin{array}{l}\text { Clinton } \\
\text { Dickson } \\
\text { Dickson } \\
\text { Dickson } \\
\text { Dickson }\end{array}$ & $\begin{array}{l}\text { QWP } \\
\text { STR } \\
\text { QWD } \\
\text { QWMP } \\
\text { SED }\end{array}$ & $\begin{array}{l}\text { CP } \\
\text { BCNP } \\
\text { CNP } \\
S\end{array}$ & OBS & CA & $\begin{array}{l}1977 . \\
7262 . \\
72 \varnothing 2 . \\
72 ø 2 . \\
72 \varnothing 2 .\end{array}$ & $\begin{array}{l}1953,58,60-63 \\
1928- \\
1944-81 \\
1973-77 \\
* * * 1973-\end{array}$ \\
\hline $\begin{array}{l}\emptyset 7331 \varnothing \emptyset \emptyset \\
\emptyset 73244 \varnothing \emptyset \\
\emptyset 73244 \varnothing \emptyset \\
\emptyset 73244 \varnothing \emptyset \\
\emptyset 73242 \varnothing \emptyset\end{array}$ & $\begin{array}{l}\text { Washita } R \mathrm{nr} \\
\text { Washita } R \mathrm{nr} \\
\text { Washita } R \mathrm{nr} \\
\text { Washita } R \mathrm{nr} \\
\text { Washita } \mathrm{nr}\end{array}$ & $\begin{array}{l}\text { Dickson } \\
\text { Foss } \\
\text { Foss } \\
\text { Foss } \\
\text { Hammon }\end{array}$ & $\begin{array}{l}\text { QWP } \\
\text { STR } \\
\text { QWP } \\
\text { QWMP } \\
\text { STR }\end{array}$ & $\begin{array}{l}\text { BCNP } \\
\text { CNP } \\
\text { CNP }\end{array}$ & OBS & $\begin{array}{l}\text { A } \\
\text { VG } \\
\text { VG } \\
\text { VG }\end{array}$ & $\begin{array}{l}7262 . \\
1551 . \\
1561 . \\
1551 . \\
1387 .\end{array}$ & $\begin{array}{l}1982- \\
* * 1956-57,61-87 \\
* * 1947-48,78-87 \\
1973-79 \\
1969-\end{array}$ \\
\hline $\begin{array}{l}\emptyset 73242 \emptyset \emptyset \\
\emptyset 73242 \varnothing \emptyset \\
\emptyset 732416 \emptyset \\
\emptyset 73285 \emptyset \emptyset \\
\emptyset 73285 \emptyset \emptyset\end{array}$ & $\begin{array}{l}\text { Washita } R \mathbf{n r} \\
\text { Washita } R \mathrm{nr} \\
\text { Washita } R \mathrm{nr} \\
\text { Washita } R \mathrm{nr} \\
\text { Washita } R \mathbf{n r}\end{array}$ & $\begin{array}{l}\text { Hammon } \\
\text { Hammon } \\
\text { Moorewood } \\
\text { Pauls Valley } \\
\text { Pauls Valley }\end{array}$ & $\begin{array}{l}\text { QWP } \\
\text { QWMP } \\
\text { QWP } \\
\text { STR } \\
\text { QWP }\end{array}$ & $\begin{array}{l}\text { CNP } \\
\text { CNP } \\
\text { CP } \\
\text { COP }\end{array}$ & $\begin{array}{l}\text { OBS } \\
\text { OBS }\end{array}$ & $\begin{array}{l}\text { VG } \\
\text { JG }\end{array}$ & $\begin{array}{l}1387 . \\
1387 . \\
5330 . \\
5330 .\end{array}$ & $\begin{array}{l}1961,70- \\
1978-79 \\
1970-72 \\
1937- \\
1952-63\end{array}$ \\
\hline $\begin{array}{l}\emptyset 73286 \emptyset \emptyset \\
\emptyset 73285 \emptyset \emptyset \\
\varnothing 731636 \emptyset \\
\emptyset 731636 \emptyset \\
\varnothing 7328 \varnothing \emptyset \emptyset\end{array}$ & $\begin{array}{l}\text { Washita } R n r \\
\text { Washita } R n r \\
\text { Washita } R n r \\
\text { Washita } n r \\
\text { Washita } n r\end{array}$ & $\begin{array}{l}\text { Pauls Valley } \\
\text { Pauls Valley } \\
\text { Reydon } \\
\text { Reydon } \\
\text { Tabler }\end{array}$ & $\begin{array}{l}\text { QWPM } \\
\text { SED } \\
\text { QWP } \\
\text { QWMP } \\
\text { STR }\end{array}$ & $\begin{array}{l}\text { CNP } \\
S \\
C P \\
C N P\end{array}$ & & & $\begin{array}{l}5330 . \\
5330 . \\
4.98 \\
4.98 \\
4706 .\end{array}$ & $\begin{array}{l}1976-79 \\
1976-78 \\
1949,62 \\
1977 \\
1940-52\end{array}$ \\
\hline $\begin{array}{l}\emptyset 7328 \emptyset \emptyset \emptyset \\
\emptyset 73134 \emptyset \emptyset \\
\emptyset 71497 \varnothing 9 \\
\varnothing 715765 \emptyset \\
\emptyset 715756 \varnothing\end{array}$ & $\begin{array}{l}\text { Washita R nr } \\
\text { Waurika Lake } \\
\text { West Clay Ck } \\
\text { West Fk nr K } \\
\text { West Fk nr K }\end{array}$ & $\begin{array}{l}\text { Tabler } \\
\text { nr Waurika } \\
\text { nr Yewed } \\
\text { nowles } \\
\text { inowles }\end{array}$ & $\begin{array}{l}\text { QWD } \\
\text { RESP } \\
\text { QWP } \\
\text { CS } \\
\text { CSR }\end{array}$ & $\begin{array}{l}C P \\
C P\end{array}$ & & $\begin{array}{l}\mathrm{C} \\
\mathrm{HG}\end{array}$ & $\begin{array}{l}4706 . \\
562 . \\
4.22 \\
4.22\end{array}$ & $\begin{array}{l}1947-52 \\
1978- \\
1962 \\
1964-85 \\
1964-76\end{array}$ \\
\hline $\begin{array}{l}\emptyset 72421 \emptyset \emptyset \\
\varnothing 72421 \emptyset \emptyset \\
\emptyset 7242 \varnothing 5 \emptyset \\
\emptyset 715 \varnothing 59 \emptyset \\
\emptyset 724237 \emptyset\end{array}$ & $\begin{array}{l}\text { Wewoka Ck nr } \\
\text { Wewoka Ck } n r \\
\text { Wewoka Ck nr } \\
\text { Wild Horse } \\
\text { Wildhorse Ck }\end{array}$ & $\begin{array}{l}\text { Wetumka } \\
\text { Wetumka } \\
\text { Wewoka } \\
\text { Sand) Ck nr Pond Creek } \\
\text { Trib nr Luther }\end{array}$ & $\begin{array}{l}\text { STR } \\
\text { QWP } \\
\text { QWP } \\
\text { LF } \\
\text { CSR }\end{array}$ & $\begin{array}{l}\mathrm{CP} \\
\mathrm{CP}\end{array}$ & & & $\begin{array}{l}396 . \\
396 . \\
165 . \\
2.12\end{array}$ & $\begin{array}{l}1960-63,66-67 \\
1952-67,6 \emptyset-64 \\
1962-63 \\
1965-72 \\
1974-76\end{array}$ \\
\hline $\begin{array}{l}\emptyset 724237 \emptyset \\
\emptyset 732966 \emptyset \\
\emptyset 73297 \emptyset \emptyset \\
\emptyset 73297 \emptyset \emptyset \\
\emptyset 732586 \emptyset\end{array}$ & $\begin{array}{l}\text { Wi Idhorse Ck } \\
\text { Wildhorse Ck } \\
\text { Wildhorse Ck } \\
\text { Wi Idhorse Ck } \\
\text { Willow Ck nr }\end{array}$ & $\begin{array}{l}\text { Trib nr Luther } \\
\text { nr Hennepin } \\
\text { nr Hoover } \\
\text { nr Hoover } \\
\text { Albert }\end{array}$ & $\begin{array}{l}\text { CS } \\
\text { QWP } \\
\text { STR } \\
\text { QWD } \\
\text { STR }\end{array}$ & $\begin{array}{l}C P \\
C P\end{array}$ & & $\begin{array}{l}J G \\
J G\end{array}$ & $\begin{array}{l}2.12 \\
604 . \\
604 . \\
28.0\end{array}$ & $\begin{array}{l}1974- \\
1949-5 \emptyset \\
1969- \\
1951-59, * 7 \varnothing-71 \\
197 \varnothing-78\end{array}$ \\
\hline
\end{tabular}

* Continuous streamflow records for this period.

** Some records in intervening years.

*** Some records may have been collected previously. 
Table 1.--Alphabetical listing of current and historical gaging stations maintained by the U.S. Geological Survey

September 30, 1987--Continued

[Symbols defined on last page of report]

\begin{tabular}{|c|c|c|c|c|c|c|c|}
\hline $\begin{array}{c}\text { Station } \\
\text { Number }\end{array}$ & Station & $\begin{array}{l}\text { Type } \\
\text { of } \\
\text { Data }\end{array}$ & $\begin{array}{l}\text { Q.W. } \\
\text { Para- } \\
\text { meters }\end{array}$ & $\begin{array}{l}\text { Stage } \\
\text { Source }\end{array}$ & $\begin{array}{l}\text { Coop- } \\
\text { erator }\end{array}$ & $\begin{array}{c}\text { Drainage } \\
\text { Area } \\
\left(m i l e^{2}\right)\end{array}$ & Period of Record \\
\hline $\begin{array}{l}\emptyset 731613 \emptyset \\
\emptyset 7328 \varnothing 7 \emptyset \\
\emptyset 7248 \emptyset \emptyset \emptyset \\
\emptyset 7248 \varnothing \emptyset \emptyset \\
\emptyset 7236 \varnothing 6 \emptyset\end{array}$ & $\begin{array}{l}\text { Wilson Ck Trib nr McMillan } \\
\text { Winter Ck nr Alex } \\
\text { Wister Lake nr Wister } \\
\text { Wister Lake nr Wister } \\
\text { Wolf Ck Trib nr Tangier }\end{array}$ & $\begin{array}{l}\text { CS } \\
\text { STRP } \\
\text { RES } \\
\text { QWP } \\
\text { CS }\end{array}$ & $C P$ & $D C P$ & $\begin{array}{l}J G \\
C\end{array}$ & $\begin{array}{l}2.97 \\
33.6 \\
993 . \\
993 . \\
6.23\end{array}$ & $\begin{array}{l}1965-75 \\
1964-1987 \\
1949- \\
1960-64 \\
1964-72\end{array}$ \\
\hline 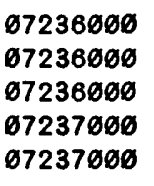 & $\begin{array}{l}\text { Wolf Ck nr Fargo } \\
\text { Wolf Ck nr Fargo } \\
\text { Wolf Ck nr Fargo } \\
\text { Wolf Ck nr Fort Supply } \\
\text { Wolf Ck nr Fort Supply }\end{array}$ & $\begin{array}{l}\text { STR } \\
\text { QWP } \\
\text { SED } \\
\text { STR } \\
\text { QWP }\end{array}$ & $\begin{array}{l}\mathrm{CP} \\
\mathrm{S} \\
\mathrm{CP}\end{array}$ & OBS & C & $\begin{array}{l}1624 . \\
1624 \\
1624 \\
1739 . \\
1739 .\end{array}$ & $\begin{array}{l}1942-76 \\
1958,60-63 \\
* * * 1973-76 \\
1937- \\
1951-58,60\end{array}$ \\
\hline $\begin{array}{l}\emptyset 7237 \varnothing \varnothing \emptyset \\
\emptyset 718948 \emptyset \\
\emptyset 72355 \varnothing \emptyset \\
\emptyset 722893 \emptyset \\
\emptyset 733852 \emptyset\end{array}$ & $\begin{array}{l}\text { Wolf Ck nr Fort Supply } \\
\text { Wolf Ck nr Grove } \\
\text { Wolf Ck } n r \text { Shattuck } \\
\text { Worley Ck nr Tuttle } \\
\text { Yanubbee Ck nr Broken Bow }\end{array}$ & $\begin{array}{l}\text { SED } \\
\text { LF } \\
\text { STRP } \\
\text { CS } \\
\text { CSR }\end{array}$ & s & & $\begin{array}{l}H G \\
H G\end{array}$ & $\begin{array}{c}1739 . \\
7.21 \\
1183 . \\
11.2 \\
9.16\end{array}$ & $\begin{array}{l}1976 \\
1966-72 \\
1938-46 \\
1965-72,75-85 \\
1964-76\end{array}$ \\
\hline 07338520 & Yanubbee Ck nr Broken Bow & CS & & & $H G$ & 9.10 & $1964-86$ \\
\hline
\end{tabular}

* Continuous streamflow records for this period.

** Some records in intervening years.

*** Some records may have been collected previously. 
Table 2.--Station number listing of current and historical gaging stations maintained by the U.S. Geological Survey

September 90, 1987

[Symbols defined on last page of report]

\begin{tabular}{|c|c|c|c|c|c|c|c|}
\hline $\begin{array}{l}\text { Station } \\
\text { Number }\end{array}$ & Station & $\begin{array}{l}\text { Type } \\
\text { of } \\
\text { Data }\end{array}$ & $\begin{array}{l}\text { Q.W. } \\
\text { Para- } \\
\text { meters }\end{array}$ & $\begin{array}{l}\text { Stage } \\
\text { Source }\end{array}$ & $\begin{array}{l}\text { Coop- } \\
\text { erator }\end{array}$ & $\begin{array}{c}\text { Drainage } \\
\text { Area } \\
(\mathrm{mile})\end{array}$ & Period of Record \\
\hline $\begin{array}{l}\emptyset 71465 \emptyset \emptyset \\
\emptyset 71465 \emptyset \emptyset \\
\emptyset 71465 \emptyset 0 \\
\emptyset 714780 \emptyset \\
\emptyset 7148126\end{array}$ & $\begin{array}{l}\text { Arkansas } R \text { at Arkansas City KS } \\
\text { Arkansas } R \text { at Arkansas City KS } \\
\text { Arkansas } R \text { at Arkansas City KS } \\
\text { Walnut } R \text { at Winfield KS } \\
\text { Beaver Ck nr Kaw City }\end{array}$ & $\begin{array}{l}\text { STRP } \\
\text { QWD } \\
\text { QWMP } \\
\text { QWP } \\
\text { QWP }\end{array}$ & $\begin{array}{l}\text { CNP } \\
\text { CNP } \\
C \\
\text { COP }\end{array}$ & BDT & $W$ & $\begin{array}{c}43713 \\
43713 \\
43713 \\
1872\end{array}$ & $\begin{array}{l}1955-76 \\
1952- \\
1972-75 \\
196 \varnothing-61 \\
195 \varnothing, 54-65\end{array}$ \\
\hline $\begin{array}{l}\emptyset 7148128 \\
\emptyset 714813 \emptyset \\
\emptyset 714814 \emptyset \\
\emptyset 714814 \emptyset \\
\emptyset 714814 \emptyset\end{array}$ & $\begin{array}{l}\text { Arkansas } R \text { at Kaw City } \\
\text { Kaw Lake } n r \text { Ponca City } \\
\text { Arkansas } R \mathrm{nr} \text { Ponca City } \\
\text { Arkansas } R \mathrm{nr} \text { Ponca City } \\
\text { Arkansas } R \mathrm{nr} \text { Ponca City }\end{array}$ & $\begin{array}{l}\text { QWD } \\
\text { RES } \\
\text { STR } \\
\text { QWMP } \\
\text { SED }\end{array}$ & $\begin{array}{l}\text { CNP } \\
\text { CNP } \\
S\end{array}$ & $D C P$ & $\begin{array}{l}c \\
c \\
c\end{array}$ & $\begin{array}{l}8670 . \\
46530 \\
46530 \\
46530 \\
46530\end{array}$ & $\begin{array}{l}1949-51 \\
1977- \\
1977- \\
1978-79 \\
1978-\end{array}$ \\
\hline $\begin{array}{l}\emptyset 714835 \emptyset \\
\emptyset 714835 \emptyset \\
\emptyset 714835 \emptyset \\
\emptyset 714836 \emptyset \\
\emptyset 7148379\end{array}$ & $\begin{array}{l}\text { Salt Fk Arkansas } R \text { nr Winchester } \\
\text { Salt Fk Arkansas } R \text { nr Winchester } \\
\text { Salt Fk Arkansas } R n r \text { Winchester } \\
\text { Greenwood Ck } n r \text { Winchester } \\
\text { Turkey Ck } n r \text { Alva }\end{array}$ & $\begin{array}{l}\text { STR } \\
\text { QWD } \\
\text { QWMP } \\
\text { LF } \\
\text { QWP }\end{array}$ & $\begin{array}{l}\text { CNP } \\
\text { CNP } \\
\text { CNP }\end{array}$ & & JG & $\begin{array}{l}856 . \\
856 . \\
856 . \\
41.2\end{array}$ & $\begin{array}{l}1959- \\
1959-62 \\
1976-77 \\
1972- \\
1962\end{array}$ \\
\hline $\begin{array}{l}\emptyset 71484 \emptyset \emptyset \\
\emptyset 71484 \emptyset \emptyset \\
\emptyset 71484 \emptyset \emptyset \\
\emptyset 714845 \emptyset \\
\emptyset 714846 \emptyset\end{array}$ & $\begin{array}{l}\text { Salt Fk Arkansas } R n r \text { Alva } \\
\text { Salt Fk Arkansas } R n r \text { Alva } \\
\text { Salt Fk Arkansas } R n r \text { Alva } \\
\text { Salt Fk Arkansas } R n r \text { Ingersoll } \\
\text { Salt Fk Arkansas } R n r \text { Ingersoll }\end{array}$ & $\begin{array}{l}\text { STR } \\
\text { QWP } \\
\text { SED } \\
\text { STR } \\
\text { QWH }\end{array}$ & $\begin{array}{l}\text { CNP } \\
S \\
\text { CNP }\end{array}$ & & $\begin{array}{l}c \\
c\end{array}$ & $\begin{array}{l}1609 . \\
1609 . \\
1609 . \\
1140 . \\
1140 .\end{array}$ & $\begin{array}{l}* 1938-51,76- \\
1950-54,62,76-79 \\
1979- \\
1961-62,73-79 \\
1961-63,73-79\end{array}$ \\
\hline $\begin{array}{l}\emptyset 714845 \emptyset \\
\emptyset 714845 \emptyset \\
\varnothing 71496 \varnothing \emptyset \\
\emptyset 71490 \emptyset \emptyset \\
\emptyset 71493 \emptyset 2\end{array}$ & $\begin{array}{l}\text { Salt Fk Arkansas R nr Ingersoll } \\
\text { Salt Fk Arkansas R nr Ingersoll } \\
\text { Medicine Lodge R nr Kiowa, KS } \\
\text { Medicine Lodge R nr Kiowa, KS } \\
\text { Medicine Lodge R nr Driftwood }\end{array}$ & $\begin{array}{l}\text { QWMP } \\
\text { SED } \\
\text { QWH } \\
\text { QWMP } \\
\text { QWP }\end{array}$ & $\begin{array}{l}\text { CNP } \\
S \\
C P \\
\text { CNP } \\
C P\end{array}$ & & $w$ & $\begin{array}{c}1140 . \\
1140 . \\
903 . \\
903 .\end{array}$ & $\begin{array}{l}1975-79 \\
* * * 1973-79 \\
1955-57,60-62,73- \\
1976-77 \\
1962\end{array}$ \\
\hline $\begin{array}{l}\emptyset 714950 \emptyset \\
\emptyset 71496 \emptyset 8 \\
\emptyset 71497 \emptyset 0 \\
\emptyset 71497 \emptyset 9 \\
\varnothing 71499 \varnothing \varnothing\end{array}$ & $\begin{array}{l}\text { Salt FK Arkansas } R n r \text { Cherokee } \\
\text { Sandy Ck nr Ingersoll } \\
\text { Sandy Ck nr Byron } \\
\text { West Clay Ck nr Yewed } \\
\text { Great Salt Plains NADP Site nr Jet }\end{array}$ & $\begin{array}{l}\text { STRP } \\
\text { QWP } \\
\text { LF } \\
\text { QWP } \\
\text { QWP }\end{array}$ & $\begin{array}{l}\mathrm{CP} \\
\mathrm{CP} \\
\mathrm{CP}\end{array}$ & & A & $\begin{array}{c}2439 . \\
434 .\end{array}$ & $\begin{array}{l}1941-50 \\
1962 \\
1965-73 \\
1962 \\
1984-\end{array}$ \\
\hline 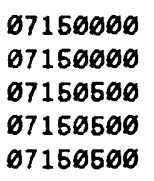 & $\begin{array}{l}\text { Great Salt Plains Lake } n r \text { Jet } \\
\text { Great Salt Plains Lake } n r \text { Jet } \\
\text { Salt Fk Arkansas } R n r \text { Jet } \\
\text { Salt Fk Arkansas } R n r \text { Jet } \\
\text { Salt Fk Arkansas } R \text { n Jet }\end{array}$ & $\begin{array}{l}\text { RES } \\
\text { QWP } \\
\text { STR } \\
\text { QWH } \\
\text { QWMP }\end{array}$ & $\begin{array}{l}\mathrm{CP} \\
\mathrm{CP} \\
\mathrm{CNP}\end{array}$ & $\begin{array}{l}D C P \\
D C P\end{array}$ & $\begin{array}{l}c \\
c \\
c\end{array}$ & $\begin{array}{l}3200 . \\
3200 . \\
3202 . \\
3202 \text {. } \\
3202 \text {. }\end{array}$ & $\begin{array}{l}1941- \\
195 \emptyset \\
1937- \\
1951-63,68- \\
1973-79\end{array}$ \\
\hline $\begin{array}{l}\emptyset 7150500 \\
\emptyset 7150500 \\
07150500 \\
07156548 \\
07150580\end{array}$ & $\begin{array}{l}\text { Salt Fk Arkansas } R \text { nr Jet } \\
\text { Salt Fk Arkansas } R \text { nr Jet } \\
\text { Salt Fk Arkansas } R n r \text { Jet } \\
\text { Sand Ck nr Hawley } \\
\text { Sand Ck Trib nr Kremlin }\end{array}$ & $\begin{array}{l}\text { SED } \\
\text { QWP } \\
\text { QWD } \\
\text { QWP } \\
\text { CS }\end{array}$ & $\begin{array}{l}S \\
C N P \\
P \\
C P\end{array}$ & OBS & $\begin{array}{l}c \\
c \\
c\end{array}$ & $\begin{array}{l}3202 . \\
3202 . \\
3202 . \\
7.21\end{array}$ & $\begin{array}{l}* * * 1973- \\
1951-6368- \\
1954-59,61-63,68- \\
1962 \\
1964-74\end{array}$ \\
\hline
\end{tabular}

* Continuous streamflow records for this period.

** Some records in intervening years.

*** Some records may have been collected previously. 
Table 2.--Station number listing of current and historical gaging stations maintained by the U.S. Geological Survey

September 30, 1987--Cont inued

[Symbols defined on last page of report]

\begin{tabular}{|c|c|c|c|c|c|c|c|}
\hline $\begin{array}{c}\text { Station } \\
\text { Number }\end{array}$ & Station & $\begin{array}{l}\text { Type } \\
\text { of } \\
\text { Data }\end{array}$ & $\begin{array}{l}\text { Q.W. } \\
\text { Para- } \\
\text { meters }\end{array}$ & $\begin{array}{l}\text { Stage } \\
\text { Source }\end{array}$ & $\begin{array}{l}\text { Coop- } \\
\text { erator }\end{array}$ & $\begin{array}{c}\text { Drainage } \\
\text { Area } \\
\left(\mathrm{mi} l e^{2}\right)\end{array}$ & Period of Record \\
\hline 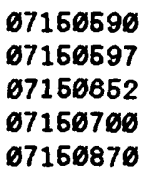 & $\begin{array}{l}\text { Wild Horse (Sand) Ck } n r \text { Pond Creek } \\
\text { Salt Fk Arkansas } R \text { nr Pond Creek } \\
\text { Osage Ck nr Medford } \\
\text { Pond Ck nr Lamont } \\
\text { Salt Fk Arkansas R Trib nr Eddy }\end{array}$ & $\begin{array}{l}\text { LF } \\
\text { QWP } \\
\text { QWP } \\
\text { QWP } \\
\text { CS }\end{array}$ & $\begin{array}{l}C P \\
C P \\
C P\end{array}$ & & $H G$ & 2.36 & $\begin{array}{l}1966-72 \\
1961,62 \\
1982 \\
1961-55,58-59,62 \\
1964-\end{array}$ \\
\hline $\begin{array}{l}\varnothing 7160900 \\
\varnothing 7160900 \\
\varnothing 7161000 \\
\varnothing 7161000 \\
\varnothing 7161000\end{array}$ & $\begin{array}{l}\text { Deer Ck nr Tonkawa } \\
\text { Deer Ck } n r \text { Tonkawa } \\
\text { Salt Fk Arkansas } R \text { at Tonkawa } \\
\text { Salt Fk Arkansas } R \text { at Tonkawa } \\
\text { Salt Fk Arkansas } R \text { at Tonkawa }\end{array}$ & $\begin{array}{l}\text { LF } \\
\text { QWP } \\
\text { STR } \\
\text { QWH } \\
\text { QWMP }\end{array}$ & $\begin{array}{l}\mathrm{CP} \\
\text { CNP } \\
\text { CNP }\end{array}$ & & $C$ & $\begin{array}{l}160 . \\
160 . \\
4628 . \\
4628 . \\
4628 .\end{array}$ & $\begin{array}{l}1965-73 \\
1968-59,62 \\
1935- \\
1948,52-63,68-79 \\
1973-77\end{array}$ \\
\hline $\begin{array}{l}\varnothing 716166 \varnothing \\
\varnothing 7161900 \\
\varnothing 7161903 \\
\varnothing 7162000 \\
\varnothing 7162000\end{array}$ & $\begin{array}{l}\text { Chikaskia } R \text { nr Drury KS } \\
\text { Chikaskia } R \text { nr Braman } \\
\text { Chikaskia } R \text { blw Braman Lake nr Braman } \\
\text { Chikaskia R } n r \text { Blackwell } \\
\text { Chikaskia R nr Blackwell }\end{array}$ & $\begin{array}{l}\text { QWP } \\
\text { QWMP } \\
\text { QWP } \\
\text { STR } \\
\text { QWP }\end{array}$ & $\begin{array}{l}\mathrm{C} \\
\mathrm{CNP} \\
\mathrm{CP} \\
\mathrm{CP}\end{array}$ & DCP & JGC & $\begin{array}{l}1610 . \\
1869 . \\
1869 .\end{array}$ & $\begin{array}{l}1968-61 \\
1977 \\
1968,69,62 \\
1936- \\
1962-63\end{array}$ \\
\hline $\begin{array}{l}\emptyset 7162000 \\
\varnothing 716206 \varnothing \\
\varnothing 7162069 \\
\varnothing 7152200 \\
\varnothing 7162260\end{array}$ & $\begin{array}{l}\text { Chikaskia } R \text { nr Blackwell } \\
\text { Chikaskia } R \text { nr Tonkawa } \\
\text { Duck Ck nr Tonkawa } \\
\text { Salt Fk Arkansas R nr Marland } \\
\text { Bois D'Arc Ck nr Ponca City }\end{array}$ & $\begin{array}{l}\text { QWMP } \\
\text { QWP } \\
\text { QWP } \\
\text { QWP } \\
\text { QWP }\end{array}$ & $\begin{array}{l}\text { CNP } \\
C P \\
C P \\
\text { CNP } \\
C P\end{array}$ & & & 1869. & $\begin{array}{l}1976-79 \\
1948,51,63,68-63 \\
1963,62 \\
1960-63 \\
1953,68-63\end{array}$ \\
\hline $\begin{array}{l}\emptyset 716226 \emptyset \\
\varnothing 715226 \emptyset \\
\varnothing 716229 \emptyset \\
6715235 \emptyset \\
\varnothing 715236 \varnothing\end{array}$ & $\begin{array}{l}\text { Bois D'Arc Ck nr Ponca City } \\
\text { Salt Fk Arkansas } R \text { nr White Eagle } \\
\text { Greasy Ck nr Watchorn } \\
\text { Red Rock nr Red Rock } \\
\text { EIm Ck nr Foraker }\end{array}$ & $\begin{array}{l}\text { LF } \\
\text { QWMP } \\
\text { STR } \\
\text { QWP } \\
\text { CS }\end{array}$ & $\begin{array}{l}\text { CNP } \\
\text { CNP }\end{array}$ & RES & & $\begin{array}{l}190 . \\
28.0 \\
18.2\end{array}$ & $\begin{array}{l}1966-73 \\
1978-79 \\
1974-76 \\
1961-59,62-63 \\
1964-75\end{array}$ \\
\hline 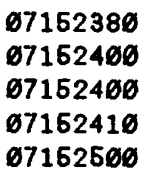 & $\begin{array}{l}\text { Beaver Ck (E Br) nr Grainola } \\
\text { Salt Ck nr Shidler } \\
\text { Salt Ck nr Shidler } \\
\text { Rock Ck nr Shidler } \\
\text { Arkansas } R \text { at Ralston }\end{array}$ & $\begin{array}{l}\text { QWP } \\
\text { LF } \\
\text { QWP } \\
\text { CS } \\
\text { STR }\end{array}$ & $\begin{array}{l}\mathrm{CP} \\
\mathrm{CP}\end{array}$ & $D C P$ & C & $\begin{array}{c}114 . \\
114 . \\
9.13 \\
54465 .\end{array}$ & $\begin{array}{l}1950 \\
1964-65,68-66 \\
1950,54-55,58,61-63 \\
1965-72 \\
1925-\end{array}$ \\
\hline 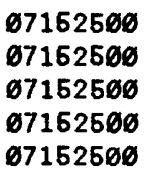 & $\begin{array}{l}\text { Arkansas } R \text { at } R a l \text { ston } \\
\text { Arkansas } R \text { at } R a l s t o n \\
\text { Arkansas } R \text { at } R a l s t o n \\
\text { Arkansas } R \text { at } R a l \text { ston } \\
\text { Arkansas } R \text { at } R a l s t o n\end{array}$ & $\begin{array}{l}\text { QWH } \\
\text { QWMP } \\
\text { SED } \\
\text { QWP } \\
\text { QWD }\end{array}$ & $\begin{array}{l}\text { BCNP } \\
\text { CNP } \\
\text { S } \\
\text { BCNP } \\
\text { P }\end{array}$ & OBS & $\begin{array}{l}\text { CAE } \\
\text { CAE } \\
C A\end{array}$ & $\begin{array}{l}54465 \\
54465 \\
54465 \\
54465 \\
54465\end{array}$ & $\begin{array}{l}1950-63,66-80 \\
1973-79 \\
* * * 1973- \\
1981- \\
195 \varnothing-63,68-\end{array}$ \\
\hline $\begin{array}{l}\emptyset 716252 \emptyset \\
\varnothing 715252 \emptyset \\
6716259 \emptyset \\
\varnothing 716295 \emptyset \\
\varnothing 716299 \emptyset\end{array}$ & $\begin{array}{l}\text { Black Bear Ck Trib nr Garber } \\
\text { Black Bear Ck Trib nr Garber } \\
\text { Black Bear Ck nr Perry } \\
\text { Black Bear Ck nr Morrison } \\
\text { Skedee Ck at Pawnee Lake nr Pawnee }\end{array}$ & $\begin{array}{l}\text { CS } \\
\text { QWP } \\
\text { QWP } \\
\text { QWP } \\
\text { QWP }\end{array}$ & $\begin{array}{l}C P \\
C P \\
C P \\
C P\end{array}$ & & & $\begin{array}{l}0.97 \\
0.97\end{array}$ & $\begin{array}{l}1964-76 \\
1958-59 \\
196 \varnothing, 58-59 \\
1951,58-59 \\
1953-56\end{array}$ \\
\hline
\end{tabular}

* Continuous streamflow records for this period.

** Some records in intervening years.

*** Some records may have been collected previously. 
Table 2.--Station number listing of current and historical gaging stations maintained by the U.S. Geological Survey

September 90, 1987--Continued

[Symbols defined on last page of report]

\begin{tabular}{|c|c|c|c|c|c|c|c|}
\hline $\begin{array}{c}\text { Station } \\
\text { Number }\end{array}$ & Station & $\begin{array}{l}\text { Type } \\
\text { of } \\
\text { Data }\end{array}$ & $\begin{array}{l}\text { Q.W. } \\
\text { Para- } \\
\text { meters }\end{array}$ & $\begin{array}{l}\text { Stage } \\
\text { Source }\end{array}$ & $\begin{array}{l}\text { Coop- } \\
\text { erator }\end{array}$ & $\begin{array}{c}\text { Orainage } \\
\text { Area } \\
(m i l e \\
(m)\end{array}$ & Period of Record \\
\hline $\begin{array}{l}\varnothing 7153000 \\
\varnothing 7153000 \\
\varnothing 71530 \varnothing \varnothing \\
\varnothing 715300 \emptyset \\
\varnothing 7153100\end{array}$ & $\begin{array}{l}\text { Black Bear Ck at Pawnee } \\
\text { Black Bear Ck at Pawnee } \\
\text { Black Bear Ck at Pawnee } \\
\text { Black Bear Ck at Pawnee } \\
\text { Ranch Ck at Cleveland Dam nr Cleveland }\end{array}$ & $\begin{array}{l}\text { STR } \\
\text { QWP } \\
\text { QWMP } \\
\text { SED } \\
\text { STR }\end{array}$ & $\begin{array}{l}\mathrm{CP} \\
\mathrm{CNP} \\
\mathrm{S}\end{array}$ & $D C P$ & C & $\begin{array}{l}576 . \\
576 \\
576 \\
576 . \\
21.9\end{array}$ & $\begin{array}{l}1944- \\
1952-53,56-59,61-71 \\
1978-79 \\
1978- \\
1945-63\end{array}$ \\
\hline $\begin{array}{l}\emptyset 715310 \emptyset \\
\varnothing 715315 \emptyset \\
\varnothing 715440 \emptyset \\
\emptyset 71545 \varnothing \emptyset \\
\varnothing 715450 \emptyset\end{array}$ & $\begin{array}{l}\text { Ranch } \mathrm{Ck} \text { at Cleveland Dam nr Cleveland } \\
\text { Keystone Lake } n r \text { Cleveland } \\
\text { Carrizozo Ck } n r \text { Kenton } \\
\text { Cimarron } \mathrm{R} n r \text { Kenton } \\
\text { Cimarron } \mathrm{R} n \text { Kenton }\end{array}$ & $\begin{array}{l}\text { QWP } \\
\text { QWP } \\
\text { CS } \\
\text { STR } \\
\text { QWP }\end{array}$ & $\begin{array}{l}\mathrm{CP} \\
\mathrm{CP}\end{array}$ & & $A$ & $\begin{array}{l}21.9 \\
111 . \\
1106 . \\
1106 .\end{array}$ & $\begin{array}{l}1953 \\
1966-74 \\
1953-7 \varnothing \\
1950- \\
1952-63\end{array}$ \\
\hline $\begin{array}{l}\emptyset 715450 \emptyset \\
\emptyset 715450 \emptyset \\
\emptyset 715465 \emptyset \\
\emptyset 71550 \varnothing \emptyset \\
\emptyset 715510 \emptyset\end{array}$ & $\begin{array}{l}\text { Cimarron } R \mathrm{nr} \text { Kenton } \\
\text { Cimarron } R \mathrm{nr} \text { Kenton } \\
\text { Tesesquite Ck } n r \text { Kenton } \\
\text { Cimarron R abv Ute Ck nr Boise City } \\
\text { Cold Springs Ck } n r \text { Wheeless }\end{array}$ & $\begin{array}{l}\text { QWMP } \\
\text { QWP } \\
\text { CS } \\
\text { STR } \\
\text { CS }\end{array}$ & $\begin{array}{l}\text { CNP } \\
\text { CNP }\end{array}$ & & $\begin{array}{l}B \\
H G \\
H G\end{array}$ & $\begin{array}{l}1106 \\
1106 \\
25.4 \\
1955 . \\
11.0\end{array}$ & $\begin{array}{l}1977 \\
1987- \\
1964-85 \\
1905-\varnothing 7,43-54 \\
1964-85\end{array}$ \\
\hline $\begin{array}{l}\emptyset 7155200 \\
\varnothing 715550 \emptyset \\
\varnothing 715551 \emptyset \\
\emptyset 715690 \emptyset \\
\emptyset 715700 \emptyset\end{array}$ & $\begin{array}{l}\text { Cold Springs Ck nr Castaneda } \\
\text { Cimarron R nr Boise City } \\
\text { Flagg Springs Ck Trib nr Boise City } \\
\text { Cimarron R nr Forgan KS } \\
\text { Cimarron R nr Mocane }\end{array}$ & $\begin{array}{l}\text { LF } \\
\text { STR } \\
\text { CS } \\
\text { STR } \\
\text { PR }\end{array}$ & & & $J G$ & $\begin{array}{l}129 . \\
2214 . \\
5.16 \\
8536 \\
8670\end{array}$ & $\begin{array}{l}1965-71 \\
1939-42 \\
1965-72 \\
1965- \\
* 1943-65,76-79\end{array}$ \\
\hline $\begin{array}{l}\emptyset 7157 \varnothing 6 \emptyset \\
\emptyset 71575 \varnothing \emptyset \\
\emptyset 715755 \emptyset \\
\emptyset 715755 \emptyset \\
\varnothing 715758 \emptyset\end{array}$ & $\begin{array}{l}\text { Cimarron R } n r \text { Mocane } \\
\text { Crooked Ck } n r \text { Nye KS } \\
\text { West Fk } n r \text { Knowles } \\
\text { West Fk } n r \text { Knowles } \\
\text { Cimarron R nr Enlgewood KS }\end{array}$ & $\begin{array}{l}\text { QWP } \\
\text { QWP } \\
\text { CS } \\
\text { CSR } \\
\text { STR }\end{array}$ & $\begin{array}{l}C P \\
C P\end{array}$ & & $\begin{array}{l}H G \\
J G\end{array}$ & $\begin{array}{l}8670 . \\
1157 \\
4.22 \\
4.22 \\
10896\end{array}$ & $\begin{array}{l}1947-49,62-64,76-79 \\
1947,58-63 \\
1964-85 \\
1964-76 \\
1982-87\end{array}$ \\
\hline $\begin{array}{l}\emptyset 715758 \emptyset \\
\emptyset 715758 \emptyset \\
\emptyset 715774 \emptyset \\
\varnothing 715774 \emptyset \\
\varnothing 715794 \varnothing\end{array}$ & $\begin{array}{l}\text { Cimarron } R \text { nr Enlgewood KS } \\
\text { Cimarron R nr Enlgewood KS } \\
\text { Cimarron R nr Buttermilk KS } \\
\text { Cimarron R nr Buttermilk KS } \\
\text { Bluff Ck nr Buttermilk KS }\end{array}$ & $\begin{array}{l}\text { QWP } \\
\text { SED } \\
\text { STR } \\
\text { QWH } \\
\text { STR }\end{array}$ & $\begin{array}{l}\text { CNP } \\
\mathrm{S} \\
\mathrm{CP}\end{array}$ & & $\begin{array}{l}J G \\
J G\end{array}$ & $\begin{array}{l}16096 \\
10096 \\
11120 \\
11120 \\
657\end{array}$ & $\begin{array}{l}1982-87 \\
1982-87 \\
1973-79 \\
1973-79 \\
1973-79\end{array}$ \\
\hline $\begin{array}{l}\emptyset 715794 \emptyset \\
6715795 \emptyset \\
6715795 \emptyset \\
\varnothing 715795 \emptyset \\
\varnothing 7157950\end{array}$ & $\begin{array}{l}\text { Bluff Ck } n r \text { Buttermilk KS } \\
\text { Cimarron } R \text { nr Buffalo } \\
\text { Cimarron } R \text { nr Buffalo } \\
\text { Cimarron } R \text { nr Buffalo } \\
\text { Cimarron } R \text { nr Buffalo }\end{array}$ & $\begin{array}{l}\text { QWH } \\
\text { STR } \\
\text { QWD } \\
\text { QWMP } \\
\text { SED }\end{array}$ & $\begin{array}{l}\text { CP } \\
\text { BCNP } \\
\text { CNP } \\
S\end{array}$ & OBS & $A$ & $\begin{array}{l}657 \\
12004 \\
12004 \\
12004 \\
12004\end{array}$ & $\begin{array}{l}1973-79 \\
1960- \\
1953,60-63,68-79 \\
1973-77 \\
* * * 1973-\end{array}$ \\
\hline $\begin{array}{l}\emptyset 7157950 \\
\emptyset 715796 \emptyset \\
\emptyset 7157960 \\
\emptyset 715796 \emptyset \\
\emptyset 715796 \emptyset\end{array}$ & $\begin{array}{l}\text { Cimarron R nr Buffalo } \\
\text { Buffalo Ck } n r \text { Lovedale } \\
\text { Buffalo Ck } n r \text { Lovedale } \\
\text { Buffalo Ck nr Lovedale } \\
\text { Buffalo Ck nr Lovedale }\end{array}$ & $\begin{array}{l}\text { QWP } \\
\text { STR } \\
\text { QWH } \\
\text { QWMP } \\
\text { SED }\end{array}$ & $\begin{array}{l}\text { BCNP } \\
\text { CP } \\
\text { CNP } \\
S\end{array}$ & & $\begin{array}{l}J G A \\
J G\end{array}$ & $\begin{array}{r}12004 . \\
408 \\
408 \\
408 \\
408\end{array}$ & $\begin{array}{l}1980- \\
1966- \\
1974-79 \\
1976-79 \\
1978-79\end{array}$ \\
\hline
\end{tabular}

* Continuous streamflow records for this period.

** Some records in intervening years.

*** Some records may have been collected previously. 
Table 2.--Station number listing of current and historical gaging stations maintained by the U.S. Geological Survey

September 30, 1987--Continued

[Symbols defined on last page of report]

\begin{tabular}{|c|c|c|c|c|c|c|c|}
\hline $\begin{array}{r}\text { Station } \\
\text { Number }\end{array}$ & Station Name & $\begin{array}{l}\text { Type } \\
\text { of } \\
\text { Data }\end{array}$ & $\begin{array}{l}\text { Q.W. } \\
\text { Para- } \\
\text { meters }\end{array}$ & $\begin{array}{l}\text { Stage } \\
\text { Source }\end{array}$ & $\begin{array}{l}\text { Coop- } \\
\text { erator }\end{array}$ & $\begin{array}{c}\text { Drainage } \\
\text { Area } \\
\left(m i l e^{2}\right)\end{array}$ & Period of Record \\
\hline $\begin{array}{l}\varnothing 715798 \emptyset \\
6715798 \emptyset \\
\varnothing 715798 \emptyset \\
\varnothing 7158 \varnothing \varnothing \emptyset \\
\varnothing 7158 \varnothing \varnothing \emptyset\end{array}$ & $\begin{array}{l}\text { Cimarron } R \text { at Freedom } \\
\text { Cimarron } R \text { at Freedom } \\
\text { Cimarron } R \text { at Freedom } \\
\text { Cimarron } R \text { nr Waynoka } \\
\text { Cimarron } R \text { nr Waynoka }\end{array}$ & $\begin{array}{l}\text { STR } \\
\text { QWH } \\
\text { QWMP } \\
\text { STR } \\
\text { QWH }\end{array}$ & $\begin{array}{l}C P \\
C N P \\
C P\end{array}$ & & C & $\begin{array}{l}12766 \\
12766 \\
12766 \\
13334 \\
13334\end{array}$ & $\begin{array}{l}1973-80 \\
195374-80 \\
1976-77 \\
1937- \\
1951-63,68-79\end{array}$ \\
\hline 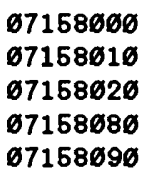 & $\begin{array}{l}\text { Cimarron R nr Waynoka } \\
\text { Main Ck nr Waynoka } \\
\text { Cimarron R Trib nr Lone Wolf } \\
\text { Sand Ck Trib nr Waynoka } \\
\text { Eagle Chief Ck nr Carmen }\end{array}$ & $\begin{array}{l}\text { QWMP } \\
\text { LF } \\
\text { CS } \\
\text { CSR } \\
\text { QWP }\end{array}$ & CNP & & & $\begin{array}{c}13334 . \\
165 . \\
4.67 \\
1.61\end{array}$ & $\begin{array}{l}1973-77 \\
1965-73 \\
1964-75 \\
1964-75 \\
1963-54\end{array}$ \\
\hline $\begin{array}{l}\emptyset 71581 \emptyset \emptyset \\
\emptyset 71581 \emptyset \emptyset \\
\emptyset 71581 \emptyset 5 \\
\emptyset 715812 \emptyset \\
\emptyset 715814 \emptyset\end{array}$ & $\begin{array}{l}\text { Eagle Chief Ck nr Aline } \\
\text { Eagle Chief Ck nr Aline } \\
\text { Eagle Chief Ck at Cleo Springs } \\
\text { Cimarron R Trib nr Isabella } \\
\text { Hoyle Ck nr Ames }\end{array}$ & $\begin{array}{l}\text { LF } \\
\text { QWP } \\
\text { QWP } \\
\text { CS } \\
\text { QWP }\end{array}$ & $\begin{array}{l}\mathrm{CP} \\
\mathrm{CP} \\
\mathrm{CP}\end{array}$ & & & $\begin{array}{l}466 . \\
466 . \\
0.62\end{array}$ & $\begin{array}{l}* * 1953-55,61-73 \\
1953-65,58-59 \\
1952-65 \\
1964-72 \\
1952-64\end{array}$ \\
\hline $\begin{array}{l}\emptyset 715815 \emptyset \\
\emptyset 715815 \emptyset \\
\emptyset 715818 \emptyset \\
\emptyset 71584 \emptyset \emptyset \\
\emptyset 71584 \emptyset \emptyset\end{array}$ & $\begin{array}{l}\text { Salt Ck nr Hitchcock } \\
\text { Salt Ck nr Hitchcock } \\
\text { Salt Ck Trib nr Okeene } \\
\text { Salt Ck nr Okeene } \\
\text { Salt Ck nr Okeene }\end{array}$ & $\begin{array}{l}\text { STR } \\
\text { QWD } \\
\text { CS } \\
\text { STR } \\
\text { QWH }\end{array}$ & $\begin{array}{l}\mathrm{CP} \\
\mathrm{CP}\end{array}$ & & & $\begin{array}{l}44.4 \\
44.4 \\
8.23 \\
196 . \\
196 .\end{array}$ & $\begin{array}{l}1960,68-7 \emptyset \\
196 \emptyset, 68-7 \emptyset \\
1964-74 \\
1961-67,73-79 \\
1973-79\end{array}$ \\
\hline $\begin{array}{l}\emptyset 71584 \emptyset \emptyset \\
\emptyset 71584 \emptyset \emptyset \\
\emptyset 715849 \emptyset \\
\emptyset 71585 \emptyset \emptyset \\
\emptyset 71585 \varnothing \emptyset\end{array}$ & $\begin{array}{l}\text { Salt Ck nr Okeene } \\
\text { Salt Ck nr Okeene } \\
\text { Preacher Ck nr Hennessey } \\
\text { Preacher Ck nr Dover } \\
\text { Preacher Ck nr Dover }\end{array}$ & $\begin{array}{l}\text { QWMP } \\
\text { SED } \\
\text { QWP } \\
\text { CS } \\
\text { QWP }\end{array}$ & $\begin{array}{l}\text { CNP } \\
S \\
C P \\
C P\end{array}$ & & HG & $\begin{array}{l}196 . \\
196 . \\
14.5 \\
14.5\end{array}$ & $\begin{array}{l}1975-79 \\
1978-79 \\
1953 \\
* 1951-67,64-85 \\
1952-54\end{array}$ \\
\hline $\begin{array}{l}\emptyset 715855 \emptyset \\
\emptyset 715855 \emptyset \\
\emptyset 7159 \emptyset \emptyset \emptyset \\
\emptyset 71596 \emptyset \emptyset \\
\emptyset 715964 \emptyset\end{array}$ & $\begin{array}{l}\text { Turkey Ck Trib nr Goltry } \\
\text { Turkey Ck Trib nr Goltry } \\
\text { Turkey Ck nr Drummond } \\
\text { Turkey Ck nr Drummond } \\
\text { Turkey Ck nr Hennessey }\end{array}$ & $\begin{array}{l}\text { CSR } \\
\text { CS } \\
\text { CS } \\
\text { QWP } \\
\text { QWP }\end{array}$ & $\begin{array}{l}C P \\
C P\end{array}$ & & $\begin{array}{l}H G \\
H G\end{array}$ & $\begin{array}{l}5 . \varnothing 8 \\
5 . \varnothing 8 \\
248 . \\
248 .\end{array}$ & $\begin{array}{l}1964-81 \\
1964-85 \\
1948-7 \varnothing, 71-74 \\
1948,52-59 \\
1951\end{array}$ \\
\hline $\begin{array}{l}\emptyset 71591 \emptyset \emptyset \\
\emptyset 71591 \emptyset \emptyset \\
\emptyset 71591 \emptyset \emptyset \\
\emptyset 71592 \emptyset \emptyset \\
\emptyset 71592 \emptyset \emptyset\end{array}$ & $\begin{array}{l}\text { Cimarron } R \mathrm{nr} \text { Dover } \\
\text { Cimarron } \mathrm{nr} \text { Dover } \\
\text { Cimarron } \mathrm{R} n \mathrm{D} \text { Dover } \\
\text { Kingfisher Ck nr Kingfisher } \\
\text { Kingfisher Ck nr Kingfisher }\end{array}$ & $\begin{array}{l}\text { STR } \\
\text { QWH } \\
\text { QWMP } \\
\text { CS } \\
\text { QWP }\end{array}$ & $\begin{array}{l}\text { CP } \\
\text { CNP } \\
C P\end{array}$ & $D C P$ & JG & $\begin{array}{r}15713 \\
15713 \\
15713 \\
157 \\
157\end{array}$ & $\begin{array}{l}1973- \\
1951,53,74-79 \\
1975-79 \\
* 1966-76,71-83 \\
1959\end{array}$ \\
\hline $\begin{array}{l}\emptyset 71592 \emptyset 3 \\
\emptyset 71594 \varnothing \emptyset \\
\emptyset 71594 \varnothing \emptyset \\
\emptyset 715945 \emptyset \\
\emptyset 71595 \emptyset \emptyset\end{array}$ & $\begin{array}{l}\text { Turkey Ck } n r \text { Dover } \\
\text { Cimarron } R \mathrm{nr} \text { Crescent } \\
\text { Cimarron } R \mathrm{nr} \text { Crescent } \\
\text { Bluff } C k \text { at OKC } \\
\text { Bluff } C k \text { abv Lake Hefner } n r \text { OKC }\end{array}$ & $\begin{array}{l}\text { QWP } \\
\text { STR } \\
\text { QWP } \\
\text { CS } \\
\text { STR }\end{array}$ & $\begin{array}{l}\mathrm{CP} \\
\mathrm{CP}\end{array}$ & & & $\begin{array}{r}16453 . \\
16453 . \\
1.64 \\
1.62\end{array}$ & $\begin{array}{l}1952-54,62 \\
197 \varnothing-72 \\
1953 \\
1974-78 \\
1950-58\end{array}$ \\
\hline
\end{tabular}

* Continuous streamflow records for this period.

** Some records in intervening years.

*** Some records may have been collected previously. 
Tablo 2.--Station number listing of current and historical gaging stations maintained by the U.S. Geological Survey

September $30,1987-$-Continued

[Symbols defined on last page of report]

\begin{tabular}{|c|c|c|c|c|c|c|c|}
\hline $\begin{array}{c}\text { Station } \\
\text { Number }\end{array}$ & Station & $\begin{array}{l}\text { Typo } \\
\text { of } \\
\text { Data }\end{array}$ & $\begin{array}{l}\text { Q.W. } \\
\text { Para- } \\
\text { meters }\end{array}$ & $\begin{array}{l}\text { Stage } \\
\text { Source }\end{array}$ & $\begin{array}{l}\text { Coop- } \\
\text { orator }\end{array}$ & $\begin{array}{c}\text { Drainage } \\
\text { Area } \\
(\mathrm{mile})\end{array}$ & Period of Record \\
\hline $\begin{array}{l}\emptyset 71596 \varnothing \emptyset \\
\emptyset 715961 \emptyset \\
\emptyset 715962 \emptyset \\
\emptyset 715963 \emptyset \\
\emptyset 715964 \emptyset\end{array}$ & $\begin{array}{l}\text { Spring } C k \text { at Lansbrook Lane, OKC } \\
\text { Spring } C k \text { at } N \text { MacArthur BIvd, OKC } \\
\text { Bluff } C k \text { abv Bethany Swg Trmt PInt } * 0 K C \\
\text { Deor Ck abv Bethany Swg Trtmt PInt } * 0 K C \\
\text { Bluff } C k \text { blw Bethany Swg Trmt PInt } * 0 K C\end{array}$ & $\begin{array}{l}\text { CSR } \\
\text { CSR } \\
\text { QWP } \\
\text { QWP } \\
\text { QWP }\end{array}$ & $\begin{array}{l}\text { CNOP } \\
\text { CNOP } \\
\text { CNOP }\end{array}$ & & & $\begin{array}{l}3.17 \\
8.43\end{array}$ & $\begin{array}{l}1976-78 \\
1976-78 \\
1983-84 \\
1983-84 \\
1983-84\end{array}$ \\
\hline $\begin{array}{l}\emptyset 7159645 \\
\emptyset 715968 \emptyset \\
\emptyset 715972 \emptyset \\
\emptyset 715972 \emptyset \\
\emptyset 715972 \emptyset\end{array}$ & $\begin{array}{l}\text { Deer Ck blw Deer Ck Swg Trtmt PInt OKC } \\
\text { Chisholm Ck at Village Dr, OKC } \\
\text { Cottonwood Ck nr Navina } \\
\text { Cottonwood Ck nr Navina } \\
\text { Cottonwood Ck nr Navina }\end{array}$ & $\begin{array}{l}\text { QWP } \\
\text { CSR } \\
\text { STR } \\
\text { QWD } \\
\text { SED }\end{array}$ & $\begin{array}{l}\text { CNOP } \\
\text { COP } \\
S\end{array}$ & OBS & $\begin{array}{l}J G \\
J G\end{array}$ & $\begin{array}{l}1.22 \\
247 . \\
247 . \\
247 .\end{array}$ & $\begin{array}{l}1983-84 \\
1976-78 \\
1978-80,82- \\
1978-8 \varnothing \\
1982-\end{array}$ \\
\hline $\begin{array}{l}\emptyset 715972 \emptyset \\
\varnothing 715972 \emptyset \\
\varnothing 715975 \emptyset \\
\varnothing 715975 \emptyset \\
\varnothing 715975 \emptyset\end{array}$ & $\begin{array}{l}\text { Cottonwood Ck } n r \text { Navina } \\
\text { Cottonwood } C k \text { nr Navina } \\
\text { Cottonwood Ck at Soward } \\
\text { Cottonwood } C k \text { at Soward } \\
\text { Cottonwood Ck at Soward }\end{array}$ & $\begin{array}{l}\text { QWP } \\
\text { QWP } \\
\text { STR } \\
\text { QWD } \\
\text { QWMP }\end{array}$ & $\begin{array}{l}\text { CP } \\
\text { COB } \\
\text { CNP } \\
\text { CNP }\end{array}$ & & $\begin{array}{l}J G \\
B\end{array}$ & $\begin{array}{l}247 \\
247 \\
316 \\
316 \\
316\end{array}$ & $\begin{array}{l}1981- \\
1984 \\
1973-82 \\
1973-82 \\
1975-79\end{array}$ \\
\hline $\begin{array}{l}\emptyset 715975 \emptyset \\
\emptyset 71598 \emptyset \emptyset \\
\emptyset 71598 \emptyset \emptyset \\
\emptyset 71598 \emptyset 5 \\
\emptyset 716 \varnothing \emptyset \emptyset \emptyset\end{array}$ & $\begin{array}{l}\text { Cottonwood Ck at Seward } \\
\text { Cottonwood Ck nr Guthrio } \\
\text { Cottonwood Ck nr Guthrie } \\
\text { Cottonwood Ck at Guthrie } \\
\text { Cimarron R nr Guthrie }\end{array}$ & $\begin{array}{l}\text { SED } \\
\text { LF } \\
\text { QWP } \\
\text { PR } \\
\text { STR }\end{array}$ & $\begin{array}{l}\mathrm{S} \\
\mathrm{CP}\end{array}$ & & $J G$ & $\begin{array}{r}316 \\
366 \\
366 \\
370 \\
16892\end{array}$ & $\begin{array}{l}1978-82 \\
1952-73 \\
1951 \\
1889-56 \\
1949,53-63,83-\end{array}$ \\
\hline $\begin{array}{l}\emptyset 716 \varnothing \emptyset \emptyset \emptyset \\
\varnothing 716 \varnothing \varnothing \emptyset \emptyset \\
\varnothing 716 \varnothing \emptyset \emptyset \emptyset \\
\emptyset 716 \emptyset 28 \emptyset \\
\emptyset 716 \varnothing 28 \emptyset\end{array}$ & $\begin{array}{l}\text { Cimarron } R n r \text { Guthrie } \\
\text { Cimarron } R n r \text { Guthrie } \\
\text { Cimarron } R n r \text { Guthrie } \\
\text { Boggy Ck at Enid Ave, Enid } \\
\text { Boggy Ck at Enid Ave, Enid }\end{array}$ & $\begin{array}{l}\text { QWD } \\
\text { QWMP } \\
\text { SED } \\
\text { STR } \\
\text { QWP }\end{array}$ & $\begin{array}{l}\text { CP } \\
\text { CNP } \\
S \\
\text { BCNP }\end{array}$ & & & $\begin{array}{l}16892 \\
16892 \\
16892 \\
20.8 \\
20.8\end{array}$ & $\begin{array}{l}1949,53-63 \\
1973-79 \\
1976 \\
1975 \\
1975\end{array}$ \\
\hline $\begin{array}{l}\emptyset 716 \varnothing 29 \emptyset \\
\varnothing 716 \varnothing 29 \emptyset \\
\varnothing 716 \varnothing 3 \emptyset \emptyset \\
\varnothing 716 \varnothing 3 \emptyset \emptyset \\
\varnothing 716 \varnothing 31 \emptyset\end{array}$ & $\begin{array}{l}\text { N Boggy Ck at Garriott Rd, Enid } \\
\text { N Boggy Ck at Garriott Rd, Enid } \\
\text { Boggy Ck at 3øth St, Enid } \\
\text { Boggy Ck at 3øth St, Enid } \\
\text { Boggy Ck Trib at Garriott Rd, Enid }\end{array}$ & $\begin{array}{l}\text { STR } \\
\text { QWP } \\
\text { STR } \\
\text { QWD } \\
\text { QWP }\end{array}$ & $\begin{array}{l}\text { BCNP } \\
\text { BCNP } \\
\text { BCNP }\end{array}$ & & & $\begin{array}{l}8.98 \\
8.98 \\
35.8 \\
35.8\end{array}$ & $\begin{array}{l}1975 \\
1975 \\
1975 \\
1975 \\
1975\end{array}$ \\
\hline $\begin{array}{l}\emptyset 716 \emptyset 316 \\
\emptyset 716 \varnothing 32 \emptyset \\
\emptyset 716 \varnothing 46 \emptyset \\
\emptyset 716 \varnothing 5 \emptyset \emptyset \\
\emptyset 716 \varnothing 5 \emptyset \emptyset\end{array}$ & $\begin{array}{l}\text { Boggy Ck abv Swg Trtmt PInt Enid } \\
\text { Boggy Ck blw Swg Trtmt Plnt Enid } \\
\text { Skeloton Ck nr Roxona } \\
\text { Skeloton Ck nr Lovell } \\
\text { Skeloton Ck nr Lovell }\end{array}$ & $\begin{array}{l}\text { QWP } \\
\text { QWP } \\
\text { QWP } \\
\text { STR } \\
\text { QWD }\end{array}$ & $\begin{array}{l}\text { BCNP } \\
\text { BCNP } \\
\text { CP } \\
\text { CP }\end{array}$ & & $J G$ & $\begin{array}{l}410 \\
410\end{array}$ & $\begin{array}{l}1976 \\
1976 \\
1950 \\
1949- \\
1951-55\end{array}$ \\
\hline $\begin{array}{l}\emptyset 716 \varnothing 5 \varnothing \emptyset \\
\varnothing 716 \varnothing 55 \emptyset \\
\emptyset 716 \varnothing 55 \emptyset \\
\varnothing 7161 \varnothing \varnothing \emptyset \\
\varnothing 7161 \varnothing \varnothing \emptyset\end{array}$ & $\begin{array}{l}\text { Skeleton Ck nr Lovell } \\
\text { W Beaver Ck } n r \text { Orlando } \\
\text { W Beaver Ck } n r \text { Orlando } \\
\text { Cimarron R at Perkins } \\
\text { Cimarron R at Perkins }\end{array}$ & $\begin{array}{l}\text { QWMP } \\
\text { CS } \\
\text { CSR } \\
\text { STR } \\
\text { QWH }\end{array}$ & $\mathrm{BCNP}$ & $D C P$ & $\begin{array}{l}H G \\
A C\end{array}$ & $\begin{array}{c}410 . \\
13.9 \\
13.9 \\
17852 \\
17852\end{array}$ & $\begin{array}{l}1976-79 \\
1964-86 \\
1964-76 \\
1939- \\
1950,53-63,69-8 \emptyset\end{array}$ \\
\hline
\end{tabular}

* Continuous streamflow records for this period.

** Some records in intervening years.

*** Some records may have been collected previously. 
Tablo 2.--Station number listing of current and historical gaging stations maintained by the U.S. Geological Survey

September 90, 1987--Continued

[Symbols defined on last page of report]

\begin{tabular}{|c|c|c|c|c|c|c|c|}
\hline $\begin{array}{r}\text { Station } \\
\text { Number }\end{array}$ & Station & $\begin{array}{l}\text { Type } \\
\text { of } \\
\text { Data }\end{array}$ & $\begin{array}{l}\text { Q.W. } \\
\text { Para- } \\
\text { meters }\end{array}$ & $\begin{array}{l}\text { Stage } \\
\text { Source }\end{array}$ & $\begin{array}{l}\text { Coop- } \\
\text { orator }\end{array}$ & $\begin{array}{c}\text { Drainage } \\
\text { Area } \\
\left(m i l e^{2}\right)\end{array}$ & Period of Record \\
\hline 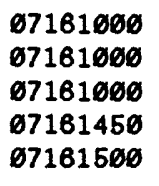 & $\begin{array}{l}\text { Cimarron } R \text { at Perkins } \\
\text { Cimarron } R \text { at Perkins } \\
\text { Cimarron } R \text { at Porkins } \\
\text { Cimarron } R \text { nr Ripley } \\
\text { Lake Carl Blackwell nr Stillwater }\end{array}$ & $\begin{array}{l}\text { QWMP } \\
\text { SED } \\
\text { QWP } \\
\text { STR } \\
\text { QWP }\end{array}$ & $\begin{array}{l}\text { CNP } \\
\text { S } \\
\text { BCNP } \\
\text { CP }\end{array}$ & DCP & $\begin{array}{l}\text { CAE } \\
\text { AE } \\
C\end{array}$ & $\begin{array}{l}17852 . \\
17852 . \\
17852 .\end{array}$ & $\begin{array}{l}1973-77 \\
* * * 1973- \\
1981- \\
1987- \\
1947,49,51,56\end{array}$ \\
\hline $\begin{array}{l}67162000 \\
67162000 \\
\varnothing 7162500 \\
\emptyset 7162500 \\
67163000\end{array}$ & $\begin{array}{l}\text { Stillwater Ck at Stillwater } \\
\text { Stillwater Ck at Still water } \\
\text { W Fk Brush Ck nr Stillwater } \\
\text { W Fk Brush Ck nr Stillwater } \\
\text { Council Ck nr Stillwater }\end{array}$ & $\begin{array}{l}\text { STR } \\
\text { SED } \\
\text { STR } \\
\text { SED } \\
\text { STR }\end{array}$ & $\begin{array}{l}\mathrm{S} \\
\mathrm{S}\end{array}$ & & JG & $\begin{aligned} 168 . \\
168 . \\
13.1 \\
13.1 \\
31.0\end{aligned}$ & $\begin{array}{l}1935-38 \\
1934-37 \\
1935-38 \\
1934-37 \\
1934-\end{array}$ \\
\hline 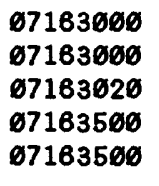 & $\begin{array}{l}\text { Council Ck nr Stilliwater } \\
\text { Council Ck nr Stilliwater } \\
\text { Corral Ck } n r \text { Yale } \\
\text { Cimarron } R \text { at Oilton } \\
\text { Cimarron } R \text { at Oilton }\end{array}$ & $\begin{array}{l}\text { QWP } \\
\text { SED } \\
\text { CS } \\
\text { CS } \\
\text { STR }\end{array}$ & $\begin{array}{l}C P \\
S\end{array}$ & & & $\begin{array}{l}31 . \emptyset \\
31 . \emptyset \\
2.89 \\
18669 . \\
18669\end{array}$ & $\begin{array}{l}1944 \\
1934-37 \\
1964-65 \\
1964-75 \\
1934-45\end{array}$ \\
\hline 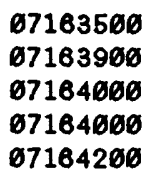 & $\begin{array}{l}\text { Cimarron } R \text { at Oilton } \\
\text { House Ck } n r \text { Terlton } \\
\text { Cimarron } R \text { at Mannford } \\
\text { Cimarron } R \text { at Mannford } \\
\text { Keystone Lake } n r \text { Sand Springs }\end{array}$ & $\begin{array}{l}\text { QWP } \\
\text { QWP } \\
\text { STR } \\
\text { QWP } \\
\text { RES }\end{array}$ & $\begin{array}{l}\mathrm{CP} \\
\mathrm{CP} \\
\mathrm{CP}\end{array}$ & $D C P$ & $\mathrm{C}$ & 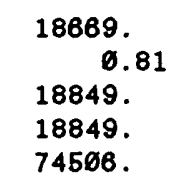 & $\begin{array}{l}1944 \\
1953-55 \\
1939-50,59-63 \\
1950-52,60-63 \\
1964-\end{array}$ \\
\hline $\begin{array}{l}\emptyset 71642 \theta \emptyset \\
\emptyset 71642 \varnothing \emptyset \\
\emptyset 716421 \emptyset \\
\emptyset 71644 \varnothing \emptyset \\
\emptyset 71644 \varnothing \emptyset\end{array}$ & $\begin{array}{l}\text { Keystone Lake nr Sand Springs } \\
\text { Keystone Lake nr Sand Springs } \\
\text { Ark } R \text { at Keystone L. TW } \\
\text { Arkansas R Sand Springs Br nr Tulsa } \\
\text { Arkansas R Sand Springs Br nr Tulsa }\end{array}$ & $\begin{array}{l}\text { QWP } \\
\text { QWMP } \\
\text { STGU } \\
\text { QWH } \\
\text { QWMP }\end{array}$ & $\begin{array}{l}\text { CP } \\
\text { COP } \\
\text { BCNP } \\
\text { CNP }\end{array}$ & $D C P$ & C & $\begin{array}{l}74566 . \\
74606 \\
74615 \\
74615\end{array}$ & $\begin{array}{l}1965-75 \\
1973-75 \\
1946-77 \\
1976-77\end{array}$ \\
\hline 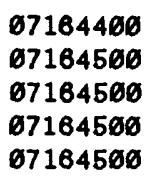 & $\begin{array}{l}\text { Arkansas } R \text { Sand Springs Br nr Tulsa } \\
\text { Arkansas } R \text { at Tulsa } \\
\text { Arkansas } R \text { at Tulsa } \\
\text { Arkansas } R \text { at Tulsa } \\
\text { Arkansas } R \text { at Tulsa }\end{array}$ & $\begin{array}{l}\text { SED } \\
\text { STR } \\
\text { QWH } \\
\text { QWMP } \\
\text { SED }\end{array}$ & $\begin{array}{l}S \\
\text { BCNP } \\
\text { CNP } \\
S\end{array}$ & $\begin{array}{l}\text { DCP } \\
\text { OBS }\end{array}$ & $\begin{array}{l}C \\
C A \\
A\end{array}$ & $\begin{array}{l}74615 \\
74615 \\
74615 \\
74615 \\
74615\end{array}$ & $\begin{array}{l}1974-77 \\
1925- \\
1960-61,77-80 \\
1977 \\
1977-\end{array}$ \\
\hline 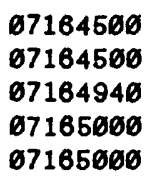 & $\begin{array}{l}\text { Arkansas } R \text { at Tulsa } \\
\text { Arkansas } R \text { at Tulsa } \\
\text { Deep Ck nr Olive } \\
\text { Heyburn Lake nr Heyburn } \\
\text { Heyburn Lake nr Heyburn }\end{array}$ & $\begin{array}{l}\text { QWP } \\
\text { QWD } \\
\text { CS } \\
\text { RES } \\
\text { QWP }\end{array}$ & $\begin{array}{l}\text { BCNP } \\
P\end{array}$ & DCP & $\begin{array}{l}\mathrm{CA} \\
\mathrm{C} \\
\mathrm{C}\end{array}$ & $\begin{array}{l}74615 . \\
74615 . \\
3.25 \\
123 . \\
123 .\end{array}$ & $\begin{array}{l}1960-61,81- \\
1977- \\
1967-72 \\
1950- \\
1961\end{array}$ \\
\hline $\begin{array}{l}\emptyset 71655 \varnothing \emptyset \\
\varnothing 71655 \varnothing \emptyset \\
\emptyset 71655 \varnothing \emptyset \\
\emptyset 71655 \varnothing 7 \\
\emptyset 716551 \emptyset\end{array}$ & $\begin{array}{l}\text { Polecat Ck at Heyburn } \\
\text { Polecat Ck at Heyburn } \\
\text { Polecat Ck at Heyburn } \\
\text { Rock Cr at Sapulpa } \\
\text { Polecat Ck nr Jenks }\end{array}$ & $\begin{array}{l}\text { STRP } \\
\text { QWP } \\
\text { SED } \\
\text { LF } \\
\text { QWP }\end{array}$ & $\begin{array}{l}\mathrm{CP} \\
\mathrm{S} \\
\mathrm{COP}\end{array}$ & & SG & $\begin{array}{c}123 . \\
123 . \\
123 . \\
67.3\end{array}$ & $\begin{array}{l}1943-79 \\
1952-53,66-68,61 \\
1978-79 \\
1979-85 \\
1960-63\end{array}$ \\
\hline
\end{tabular}

* Continuous streamflow records for this period.

** Some records in intervening years.

*** Some records may have been collected previously. 
Table 2.--Station number listing of current and historical gaging stations maintained by the U.S. Geological Survey

September 30, 1987--Continued

[Symbols defined on last page of report]

\begin{tabular}{|c|c|c|c|c|c|c|c|}
\hline $\begin{array}{l}\text { Station } \\
\text { Number }\end{array}$ & Station Name & $\begin{array}{l}\text { Type } \\
\text { of } \\
\text { Data }\end{array}$ & $\begin{array}{l}\text { Q.W. } \\
\text { Para- } \\
\text { meters }\end{array}$ & $\begin{array}{l}\text { Stage } \\
\text { Source }\end{array}$ & $\begin{array}{l}\text { Coop- } \\
\text { orator }\end{array}$ & $\begin{array}{c}\text { Drainage } \\
\text { Area } \\
(\mathrm{mile})\end{array}$ & Period of Record \\
\hline $\begin{array}{l}\emptyset 716552 \emptyset \\
\varnothing 716555 \emptyset \\
\sigma 7165559 \\
\sigma 716557 \emptyset \\
\varnothing 716557 \emptyset\end{array}$ & $\begin{array}{l}\text { Arkansas } R \text { at Bixby } \\
\text { Snake Ck } n r \text { Bixby } \\
\text { Snake Ck } n r \text { Leonard } \\
\text { Arkansas } R n r \text { Haskell } \\
\text { Arkansas } R n r \text { Haskell }\end{array}$ & $\begin{array}{l}\text { QWD } \\
\text { CS } \\
\text { QWP } \\
\text { STR } \\
\text { QWP }\end{array}$ & $\begin{array}{l}\mathrm{CP} \\
\mathrm{CP} \\
\mathrm{BCNP}\end{array}$ & DCP & C & $\begin{array}{r}50.0 \\
75473 . \\
75473 .\end{array}$ & $\begin{array}{l}1949 \\
1951-76 * * 71-76 \\
1960-61 \\
1972- \\
1974-75\end{array}$ \\
\hline $\begin{array}{l}\sigma 716557 \emptyset \\
\varnothing 716557 \emptyset \\
\sigma 7165572 \\
67165581 \\
\varnothing 71656 \varnothing \emptyset\end{array}$ & $\begin{array}{l}\text { Arkansas } R \text { nr Haskell } \\
\text { Arkansas } R \text { nr Haskell } \\
\text { Coweta Ck nr Coweta } \\
\text { Cane Ck nr Jamesville } \\
\text { Arkansas } R \text { nr Tullahassee }\end{array}$ & $\begin{array}{l}\text { QWMP } \\
\text { SED } \\
\text { QWP } \\
\text { QWP } \\
\text { STR }\end{array}$ & $\begin{array}{l}\text { CNP } \\
S \\
C P \\
C P\end{array}$ & & & $\begin{array}{l}75473 \\
75473\end{array}$ & $\begin{array}{l}1974-79 \\
1974-75 \\
1961 \\
1960-61 \\
1971-72\end{array}$ \\
\hline 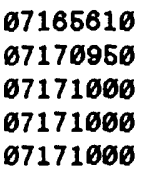 & $\begin{array}{l}\text { Arkansas } R \text { at Muskogeo } \\
\text { Verdigris } R n r \text { South Coffeyville } \\
\text { Verdigris } R n r \text { Lenapah } \\
\text { Verdigris } R \text { nr Lenapah } \\
\text { Verdigris } R \text { nr Lenapah }\end{array}$ & $\begin{array}{l}\text { QWP } \\
\text { QWP } \\
\text { STR } \\
\text { QWP } \\
\text { QWMP }\end{array}$ & $\begin{array}{l}\mathrm{CP} \\
\mathrm{CP} \\
\mathrm{CP} \\
\mathrm{CNP}\end{array}$ & DCP & C & $\begin{array}{l}4339 . \\
3639 . \\
3639 . \\
3639 .\end{array}$ & $\begin{array}{l}1957,62,63 \\
1952-53 \\
1938- \\
1946,52-64 \\
1973-79\end{array}$ \\
\hline $\begin{array}{l}67171680 \\
67171160 \\
67171165 \\
67171165 \\
67171165\end{array}$ & $\begin{array}{l}\text { California Ck nr Nowata } \\
\text { Verdigris } R \text { nr Nowata } \\
\text { E Fk Big Ck nr Hollow } \\
\text { E Fk Big Ck nr Hollow } \\
\text { E Fk Big Ck nr Hollow }\end{array}$ & $\begin{array}{l}\text { QWP } \\
\text { QWP } \\
\text { QWMP } \\
\text { PR } \\
\text { SED }\end{array}$ & $\begin{array}{l}\mathrm{CP} \\
\mathrm{CP} \\
\mathrm{CP} \\
\mathrm{S}\end{array}$ & & & $\begin{array}{l}14.4 \\
14.4 \\
14.4\end{array}$ & $\begin{array}{l}1952-53,69 \\
1952-53 \\
198 \emptyset-83 \\
198 \emptyset-83 \\
1980-83\end{array}$ \\
\hline $\begin{array}{l}\sigma 717112 \emptyset \\
\sigma 717122 \varnothing \\
\sigma 717123 \emptyset \\
\sigma 717124 \emptyset \\
\varnothing 717126 \varnothing\end{array}$ & $\begin{array}{l}\text { Clear Ck Trib nr Hollow } \\
\text { Big Ck nr Nowata } \\
\text { Salt Ck nr Alluwe } \\
\text { Lightning Ck nr Alluwe } \\
\text { Verdigris R nr Talala }\end{array}$ & $\begin{array}{l}\text { CS } \\
\text { QWP } \\
\text { QWP } \\
\text { QWP } \\
\text { QWP }\end{array}$ & $\begin{array}{l}C P \\
C P \\
C P \\
C P\end{array}$ & & HJG & 2.19 & $\begin{array}{l}1966-75,79-85 \\
1952-53,59 \\
1952-53,59 \\
1952-53,59 \\
1952-53\end{array}$ \\
\hline $\begin{array}{l}\emptyset 71713 \theta \varnothing \\
\varnothing 71714 \varnothing \emptyset \\
\sigma 71714 \varnothing \emptyset \\
\varnothing 71714 \varnothing \emptyset \\
\varnothing 7171465\end{array}$ & $\begin{array}{l}\text { Oologah Lake } n r \text { Oologah } \\
\text { Verdigris } R \mathrm{nr} \text { Oologah } \\
\text { Verdigris } R \mathrm{nr} \text { Oologah } \\
\text { Verdigris } R \mathrm{nr} \text { Oologah } \\
\text { Verdigris } R \text {, Hwy } 88 \mathrm{Br}, \mathrm{nr} \text { Claremore }\end{array}$ & $\begin{array}{l}\text { RES } \\
\text { STR } \\
\text { QWP } \\
\text { QWMP } \\
\text { QWD }\end{array}$ & $\begin{array}{l}C P \\
C N P \\
C P\end{array}$ & $D C P$ & $\begin{array}{l}c \\
c\end{array}$ & $\begin{array}{l}4339 . \\
4339 . \\
4339 . \\
4339\end{array}$ & $\begin{array}{l}1963- \\
1961- \\
1962-63,65-75 \\
1973-79 \\
1952-63,59\end{array}$ \\
\hline $\begin{array}{l}6717149 \varnothing \\
6717149 \varnothing \\
\sigma 717149 \varnothing \\
\varnothing 71716 \varnothing \emptyset \\
\varnothing 7172 \varnothing 6 \varnothing\end{array}$ & $\begin{array}{l}\text { Sweetwater Ck nr Claremore } \\
\text { Sweetwater Ck nr Claremore } \\
\text { Sweetwater Ck } n r \text { Claremore } \\
\text { Verdigris R } n r \text { Sageeyah } \\
\text { Caney R nr Elgin KS }\end{array}$ & $\begin{array}{l}\text { QWMP } \\
\text { PR } \\
\text { SED } \\
\text { STRP } \\
\text { SED }\end{array}$ & $\begin{array}{l}\mathrm{CP} \\
\mathrm{S} \\
\mathrm{CNP}\end{array}$ & & & $\begin{array}{r}4462 . \\
445 .\end{array}$ & $\begin{array}{l}198 \emptyset-83 \\
198 \emptyset-83 \\
198 \emptyset-83 \\
1939-46 \\
1973-76\end{array}$ \\
\hline 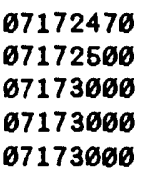 & $\begin{array}{l}\text { Caney } R n r \text { Boulangerville } \\
\text { Hulah Lake nr Hulah } \\
\text { Caney } R n r \text { Hulah } \\
\text { Caney } R \text { nr Hulah } \\
\text { Caney } R n r \text { Hulah }\end{array}$ & $\begin{array}{l}\text { QWP } \\
\text { RES } \\
\text { STR } \\
\text { QWP } \\
\text { QWMP }\end{array}$ & $\begin{array}{l}\mathrm{CP} \\
\mathrm{CNP}\end{array}$ & $D C P$ & $\begin{array}{l}\mathrm{C} \\
\mathrm{C}\end{array}$ & $\begin{array}{l}732 . \\
736 . \\
736 . \\
736 .\end{array}$ & $\begin{array}{l}1952-53 \\
1950- \\
1937- \\
1952-53, * *, 60,63-64 \\
1975-79\end{array}$ \\
\hline
\end{tabular}

* Continuous streamflow records for this period.

* Some records in intervening years.

*** Some records may have been collected previously. 
Table 2.--Station number listing of current and historical gaging stations maintained by the U.S. Geological Survey

September 30, 1987--Continued

[Symbols defined on last page of report]

\begin{tabular}{|c|c|c|c|c|c|c|c|}
\hline $\begin{array}{r}\text { Station } \\
\text { Number }\end{array}$ & Station & $\begin{array}{c}\text { Type } \\
\text { of } \\
\text { Data }\end{array}$ & $\begin{array}{l}\text { Q.W. } \\
\text { Para- } \\
\text { meters }\end{array}$ & $\begin{array}{l}\text { Stage } \\
\text { Source }\end{array}$ & $\begin{array}{l}\text { Coop- } \\
\text { erator }\end{array}$ & $\begin{array}{c}\text { Drainage } \\
\text { Area } \\
(m i l e)\end{array}$ & Period of Record \\
\hline 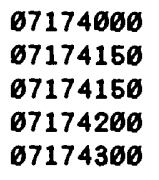 & $\begin{array}{l}\text { Caney Ck nr Copan } \\
\text { Cotton Ck nr Copan } \\
\text { Cotton Ck nr Copan } \\
\text { Little Caney R blw Cotton Ck nr Copan } \\
\text { Copan Lake nr Copan }\end{array}$ & $\begin{array}{l}\text { STRP } \\
\text { QWP } \\
\text { QWMP } \\
\text { STRP } \\
\text { RES }\end{array}$ & $\begin{array}{l}\text { CP } \\
\text { CNP }\end{array}$ & $D C P$ & C & 502. & $\begin{array}{l}1944-58 \\
1952-53,67-68 \\
1973-79 \\
1958-80 \\
1983-\end{array}$ \\
\hline $\begin{array}{l}\emptyset 717431 \emptyset \\
\emptyset 71745 \varnothing \emptyset \\
\emptyset 71745 \varnothing \emptyset \\
\emptyset 71746 \varnothing \emptyset \\
\emptyset 717461 \emptyset\end{array}$ & $\begin{array}{l}\text { Little Caney } R \text { blw Copan Lake nr Copan } \\
\text { Caney } R \text { at Bartlesville } \\
\text { Caney } R \text { at Bartlesville } \\
\text { Caney } R \text { at Bartlesville } \\
\text { Coon Ck nr Dewey }\end{array}$ & $\begin{array}{l}\text { STR } \\
\text { STRP } \\
\text { QWP } \\
\text { STR } \\
\text { QWP }\end{array}$ & $\begin{array}{l}\mathrm{CP} \\
\mathrm{CP}\end{array}$ & DCP & C & $\begin{array}{l}1465 . \\
1465 . \\
1465 .\end{array}$ & $\begin{array}{l}1984- \\
1958-56 \\
1952-53,67-68 \\
1958-56,86- \\
1952-53\end{array}$ \\
\hline $\begin{array}{l}\emptyset 717457 \emptyset \\
671746 \varnothing \emptyset \\
\emptyset 71746 \varnothing \emptyset \\
6717465 \emptyset \\
671747 \varnothing \emptyset\end{array}$ & $\begin{array}{l}\text { Dry Hollow nr Pawhuska } \\
\text { Sand } C k \text { at Okesa } \\
\text { Sand } C k \text { at Okesa } \\
\text { Sand } C k \text { at Bartlesville } \\
\text { Caney } R \text { nr Ochelata }\end{array}$ & $\begin{array}{l}\text { CS } \\
\text { STR } \\
\text { QWP } \\
\text { QWP } \\
\text { STRP }\end{array}$ & $\begin{array}{l}C P \\
C P\end{array}$ & & C & $\begin{array}{l}1.67 \\
139 . \\
139 . \\
1753 .\end{array}$ & $\begin{array}{l}1965-72 \\
1959- \\
1952-65,60-62 \\
1952-53 \\
1956-76\end{array}$ \\
\hline 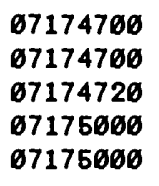 & $\begin{array}{l}\text { Caney R nr Ochelata } \\
\text { Caney R nr Ochelata } \\
\text { Hogshooter Ck Trib nr Bartlesville } \\
\text { Double Ck SWS \#5 nr Ramona } \\
\text { Double Ck SWS \#5 nr Ramona }\end{array}$ & $\begin{array}{l}\text { QWP } \\
\text { QWMP } \\
\text { CS } \\
\text { STRP } \\
\text { QWP }\end{array}$ & $\begin{array}{l}\text { CP } \\
\text { CNP }\end{array}$ & & HG & $\begin{array}{r}1753 . \\
1753 . \\
0.94 \\
2.39 \\
2.39\end{array}$ & $\begin{array}{l}1960-61 \\
1973-76 \\
1965-86 \\
1955-69 \\
1954-65, * *, 67-69\end{array}$ \\
\hline $\begin{array}{l}\emptyset 71755 \varnothing \emptyset \\
671755 \varnothing \emptyset \\
\varnothing 71755 \varnothing \emptyset \\
\emptyset 717555 \emptyset \\
\varnothing 717555 \varnothing\end{array}$ & $\begin{array}{l}\text { Caney } R \text { nr Ramona } \\
\text { Caney } R \text { nr Ramona } \\
\text { Caney R nr Ramona } \\
\text { Caney } R \text { nr Collinsville } \\
\text { Caney } R \text { nr Collinsville }\end{array}$ & $\begin{array}{l}\text { STR } \\
\text { QWP } \\
\text { QWMP } \\
\text { QWP } \\
\text { LF }\end{array}$ & $\begin{array}{l}\text { CNP } \\
\text { CNP } \\
\text { CP }\end{array}$ & $D C P$ & $\begin{array}{l}\mathrm{C} \\
J G \\
2 G\end{array}$ & $\begin{array}{l}1955 \\
1955 \\
1955\end{array}$ & $\begin{array}{l}1935-391935-3945- \\
1952-53, * * 65- \\
1975-79 \\
1949-53,59 \\
1979-84\end{array}$ \\
\hline 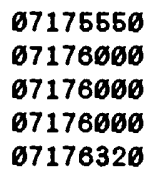 & $\begin{array}{l}\text { Caney R nr Collinsville } \\
\text { Verdigris } \mathrm{R} n \mathrm{Claremore} \\
\text { Verdigris } \mathrm{R} n \mathrm{Claremore} \\
\text { Verdigris } \mathrm{R} n \mathrm{Cl} \text { Claremore } \\
\text { Bird Ck nr Pawhuska }\end{array}$ & $\begin{array}{l}\text { STGU } \\
\text { STR } \\
\text { QWD } \\
\text { QWMP } \\
\text { QWP }\end{array}$ & $\begin{array}{l}\text { CP } \\
\text { CNP } \\
\text { CP }\end{array}$ & $\begin{array}{l}D C P \\
D C P\end{array}$ & $\begin{array}{l}c \\
c\end{array}$ & $\begin{array}{r}6534 . \\
6534 \\
6534 \\
157\end{array}$ & $\begin{array}{l}1986- \\
1935- \\
1948-54,59 \\
1978-79 \\
1962-63\end{array}$ \\
\hline $\begin{array}{l}\emptyset 717635 \emptyset \\
67176455 \\
\emptyset 717646 \varnothing \\
\emptyset 7176465 \\
\emptyset 71765 \varnothing \varnothing\end{array}$ & $\begin{array}{l}\text { Bird Ck nr Barnsdall } \\
\text { Birch Ck nr Barnsdall } \\
\text { Birch Lake nr Barnsdall } \\
\text { Birch Ck blw Birch Lake nr Barnsdall } \\
\text { Bird Ck at Avant }\end{array}$ & $\begin{array}{l}\text { QWP } \\
\text { QWP } \\
\text { RES } \\
\text { STR } \\
\text { STR }\end{array}$ & $\begin{array}{l}C P \\
C P\end{array}$ & $\begin{array}{l}D C P \\
D C P\end{array}$ & $\begin{array}{l}c \\
c \\
c\end{array}$ & $\begin{array}{l}66.0 \\
66.0 \\
364 .\end{array}$ & $\begin{array}{l}1949-53 \\
1965-66 \\
1977- \\
1978- \\
1945-\end{array}$ \\
\hline $\begin{array}{l}\emptyset 71765 \varnothing \emptyset \\
\emptyset 71765 \varnothing \emptyset \\
\emptyset 7176525 \\
\emptyset 71768 \varnothing \emptyset \\
\emptyset 717691 \varnothing\end{array}$ & $\begin{array}{l}\text { Bird } C k \text { at Avant } \\
\text { Bird } C k \text { at Avant } \\
\text { Candy } C k n r \text { Avant } \\
\text { Candy } C k n r \text { Wolco } \\
\text { Bird } C k n r \text { Skiatook }\end{array}$ & $\begin{array}{l}\text { QWP } \\
\text { QWMP } \\
\text { QWP } \\
\text { STRP } \\
\text { QWP }\end{array}$ & $\begin{array}{l}\mathrm{CP} \\
\mathrm{CNP} \\
\mathrm{CP} \\
\mathrm{CP}\end{array}$ & & & $\begin{array}{l}364 . \\
364 . \\
36.6\end{array}$ & $\begin{array}{l}1965-66 \\
1973-79 \\
1952,65-66 \\
1969-81 \\
1948-53\end{array}$ \\
\hline
\end{tabular}

* Continuous streamflow records for this period.

** Some records in intervening years.

*** Some records may have been collected previously. 
Table 2.--Station number listing of current and historical gaging stations maintained by the U.S. Geological Survey

September 90, 1987--Continued

[Symbols defined on last page of report]

\begin{tabular}{|c|c|c|c|c|c|c|c|}
\hline $\begin{array}{l}\text { Station } \\
\text { Number }\end{array}$ & Station & $\begin{array}{l}\text { Type } \\
\text { of } \\
\text { Data }\end{array}$ & $\begin{array}{l}\text { Q.W. } \\
\text { Para- } \\
\text { meters }\end{array}$ & $\begin{array}{l}\text { Stage } \\
\text { Source }\end{array}$ & $\begin{array}{l}\text { Coop- } \\
\text { orator }\end{array}$ & $\begin{array}{c}\text { Drainage } \\
\text { Area } \\
(\mathrm{mile})\end{array}$ & Period of Record \\
\hline $\begin{array}{l}97176950 \\
97177000 \\
\varnothing 717700 \emptyset \\
\varnothing 7177400 \\
\varnothing 7177410\end{array}$ & $\begin{array}{l}\text { Hominy Ck nr Hominy } \\
\text { Hominy Ck nr Skiatook } \\
\text { Hominy Ck nr Skiatook } \\
\text { Skiatook Lake nr Skiatook } \\
\text { Hominy Ck blw Skiatook Lk nr Skiatook }\end{array}$ & $\begin{array}{l}\text { QWP } \\
\text { STRP } \\
\text { QWP } \\
\text { RES } \\
\text { STR }\end{array}$ & $\begin{array}{l}\mathrm{CP} \\
\mathrm{CP}\end{array}$ & DCP & $\begin{array}{l}c \\
c\end{array}$ & $\begin{array}{l}340 . \\
340 . \\
354 . \\
364 .\end{array}$ & $\begin{array}{l}195 \emptyset-53,56,65-66 \\
1944-8 \varnothing \\
1948-53,65-66 \\
1984- \\
1984-\end{array}$ \\
\hline $\begin{array}{l}\emptyset 7177450 \\
67177500 \\
\varnothing 7177690 \\
\varnothing 7177500 \\
\varnothing 7177500\end{array}$ & $\begin{array}{l}\text { Hominy Ck nr Sperry } \\
\text { Bird Ck nr Sperry } \\
\text { Bird Ck nr Sperry } \\
\text { Bird Ck nr Sperry } \\
\text { Bird Ck nr Sperry }\end{array}$ & $\begin{array}{l}\text { QWP } \\
\text { STR } \\
\text { QWD } \\
\text { QWMP } \\
\text { QWH }\end{array}$ & $\begin{array}{l}\text { CP } \\
C P \\
\text { CNP } \\
P\end{array}$ & DCP & C & $\begin{array}{l}905 . \\
905 . \\
905 . \\
905 .\end{array}$ & $\begin{array}{l}1952-63 \\
1938- \\
1952-53, * *, 64-76 \\
1976-77 \\
1986-\end{array}$ \\
\hline 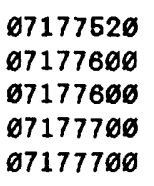 & $\begin{array}{l}\text { Delaware Ck } n r \text { Sperry } \\
\text { Bird } C k \text { at } 66 \text { th St at Tulsa } \\
\text { Bird } C k \text { at } 66 \text { th St at Tulsa } \\
\text { Flat Rock at Hwy } 75 \text { at Tulsa } \\
\text { Flat Rock at Hwy } 75 \text { at Tulsa }\end{array}$ & $\begin{array}{l}\text { QWP } \\
\text { STR } \\
\text { QWH } \\
\text { STR } \\
\text { QWH }\end{array}$ & $\begin{array}{l}C P \\
P\end{array}$ & & $\begin{array}{l}P \\
P \\
P \\
P\end{array}$ & & $\begin{array}{l}1952-53 \\
1986- \\
1986- \\
1986- \\
1987-\end{array}$ \\
\hline $\begin{array}{l}9717800 \emptyset \\
\varnothing 717800 \emptyset \\
\varnothing 71780 \varnothing \emptyset \\
6717804 \emptyset \\
9717804 \emptyset\end{array}$ & $\begin{array}{l}\text { Bird Ck nr Owasso } \\
\text { Bird } C k n r \text { Owasso } \\
\text { Bird } C k n r \text { Owasso } \\
\text { Mingo } C k \text { at } N 46 \text { th } S t \text { at Tulsa } \\
\text { Mingo } C k \text { at } N 46 \text { th } S t \text { at Tulsa }\end{array}$ & $\begin{array}{l}\text { STR } \\
\text { QWP } \\
\text { QWH } \\
\text { STR } \\
\text { QWH }\end{array}$ & $\begin{array}{l}C P \\
P \\
P\end{array}$ & & $\begin{array}{l}P \\
P \\
P \\
P\end{array}$ & $\begin{array}{l}1622 . \\
1622 . \\
1622 .\end{array}$ & $\begin{array}{l}1936-39,86- \\
1948-53 \\
1986- \\
1987- \\
1987-\end{array}$ \\
\hline $\begin{array}{l}\emptyset 7178 ø 5 \emptyset \\
\emptyset 7178 \emptyset 5 \emptyset \\
\emptyset 71784 \varnothing \emptyset \\
\emptyset 717845 \emptyset \\
\emptyset 71785 \emptyset \emptyset\end{array}$ & $\begin{array}{l}\text { Bird Ck nr Catoosa } \\
\text { Bird Ck nr Catoosa } \\
\text { Bird Ck at Catoosa } \\
\text { Verdigris } R \text { at Catoosa } \\
\text { Verdigris } R \text { nr Catoosa }\end{array}$ & $\begin{array}{l}\text { QWP } \\
\text { QWMP } \\
\text { QWMP } \\
\text { STGU } \\
\text { STR }\end{array}$ & $\begin{array}{l}\text { CP } \\
\text { CNP } \\
\text { CNP }\end{array}$ & DCP & $J G$ & $\begin{array}{l}1080 . \\
1080 . \\
7709 .\end{array}$ & $\begin{array}{l}1965- \\
1975-79 \\
1978-79 \\
1903-\varnothing 5\end{array}$ \\
\hline $\begin{array}{l}\emptyset 717856 \emptyset \\
9717858 \emptyset \\
971786 \emptyset \emptyset \\
\emptyset 71786 \emptyset \emptyset \\
971786 \varnothing \emptyset\end{array}$ & $\begin{array}{l}\text { Dog Ck nr Claremore } \\
\text { Otter Ck nr Tiawah } \\
\text { Verdigris R nr Inola } \\
\text { Verdigris } R \text { nr Inola } \\
\text { Verdigris R nr Inola }\end{array}$ & $\begin{array}{l}\text { LF } \\
\text { CS } \\
\text { STRP } \\
\text { QWP } \\
\text { QWMP }\end{array}$ & $\begin{array}{l}\text { BCNP } \\
\text { CNP }\end{array}$ & & $2 G$ & $\begin{array}{r}63.6 \\
15.2 \\
7911 \\
7911 \\
7911\end{array}$ & $\begin{array}{l}1979-84 \\
1966-72 \\
1945-7 \varnothing \\
1948-72 \\
1977\end{array}$ \\
\hline $\begin{array}{l}\emptyset 71786 \emptyset \emptyset \\
\varnothing 71786 \emptyset \emptyset \\
\varnothing 717862 \emptyset \\
\emptyset 717862 \emptyset \\
\varnothing 717862 \emptyset\end{array}$ & $\begin{array}{l}\text { Verdigris R nr Inola } \\
\text { Verdigris R nr Inola } \\
\text { Newt Graham L D nr Inola } \\
\text { Newt Graham L D nr Inola } \\
\text { Newt Graham L D nr Inola }\end{array}$ & $\begin{array}{l}\text { SED } \\
\text { STGU } \\
\text { QWP } \\
\text { QWMP } \\
\text { SED }\end{array}$ & $\begin{array}{l}S \\
\text { BCNP } \\
\text { CNP } \\
S\end{array}$ & DCP & $\begin{array}{l}C \\
A \\
A\end{array}$ & $\begin{array}{l}7911 \\
7911 \\
8630 \\
8030 \\
8030\end{array}$ & $\begin{array}{l}1948-72 \\
1986- \\
1971- \\
1973-77 \\
1974-\end{array}$ \\
\hline $\begin{array}{l}\emptyset 717862 \emptyset \\
\emptyset 7178626 \\
\emptyset 717864 \emptyset \\
\varnothing 7178646 \\
\varnothing 717865 \emptyset\end{array}$ & $\begin{array}{l}\text { Newt Graham L D nr Inola } \\
\text { Newt Graham L D nr Inola } \\
\text { Bull Ck nr Inola } \\
\text { Chouteau L D nr Chouteau (US) } \\
\text { Billy Ck Trib nr Wagoner }\end{array}$ & $\begin{array}{l}\text { RESU } \\
\text { STGU } \\
\text { CS } \\
\text { STGU } \\
\text { CS }\end{array}$ & & $D C P$ & $\begin{array}{l}c \\
c \\
c\end{array}$ & $\begin{array}{l}8030 . \\
16.7 \\
5.71\end{array}$ & $\begin{array}{l}1986- \\
1986- \\
1965-75 \\
1986- \\
1966-72\end{array}$ \\
\hline
\end{tabular}

* Continuous streamflow records for this period.

* Some records in intervening years.

*** Some records may have been collected previously. 
Table 2.--Station number listing of current and historical gaging stations maintained by the U.S. Geological Survey

September 30, 1987--Cont inued

[Symbols defined on last page of report]

\begin{tabular}{|c|c|c|c|c|c|c|c|}
\hline $\begin{array}{r}\text { Station } \\
\text { Number }\end{array}$ & Station & $\begin{array}{l}\text { Type } \\
\text { of } \\
\text { Data }\end{array}$ & $\begin{array}{l}\text { Q.W. } \\
\text { Para- } \\
\text { meters }\end{array}$ & $\begin{array}{l}\text { Stage } \\
\text { Source }\end{array}$ & $\begin{array}{l}\text { Coop- } \\
\text { erator }\end{array}$ & $\begin{array}{c}\text { Drainage } \\
\text { Area } \\
\left(m i l e^{2}\right)\end{array}$ & Period of Record \\
\hline $\begin{array}{l}07178670 \\
07178670 \\
07185000 \\
\emptyset 7185000 \\
07185000\end{array}$ & $\begin{array}{l}\text { Chouteau L D } n r \text { Chouteau (DS) } \\
\text { Chouteau L D nr Chouteau (DS) } \\
\text { Neosho R } n r \text { Commerce } \\
\text { Neosho R nr Commerce } \\
\text { Neosho R nr Commerce }\end{array}$ & $\begin{array}{l}\text { QWP } \\
\text { STGU } \\
\text { STR } \\
\text { QWP } \\
\text { SED }\end{array}$ & $\begin{array}{l}\mathrm{CP} \\
\mathrm{CNP} \\
\mathrm{S}\end{array}$ & $\begin{array}{l}\text { DCP } \\
\text { DCP }\end{array}$ & $\begin{array}{l}\text { C } \\
\text { LG } \\
C\end{array}$ & $\begin{array}{l}5876 . \\
5876 . \\
5876\end{array}$ & $\begin{array}{l}1952-53,60-63 \\
1986- \\
1939- \\
1948-54,60-73 \\
1978-\end{array}$ \\
\hline $\begin{array}{l}07185000 \\
\emptyset 7185095 \\
07185095 \\
\emptyset 7185100 \\
07188000\end{array}$ & $\begin{array}{l}\text { Neosho } R \text { nr Commerce } \\
\text { Tar Ck at 22nd St at Miami } \\
\text { Tar Ck at } 22 \text { nd St at Miami } \\
\text { Tar Ck at Miami } \\
\text { Spring } R \text { nr Quapaw }\end{array}$ & $\begin{array}{l}\text { QWMP } \\
\text { STR } \\
\text { QWP } \\
\text { STR } \\
\text { STR }\end{array}$ & $\begin{array}{l}\text { CNP } \\
\text { CNOP } \\
\mathrm{S}\end{array}$ & DCP & $\begin{array}{l}J G \\
J \\
J G \\
L G C\end{array}$ & $\begin{array}{r}5876 . \\
44.7 \\
44.7 \\
52.0 \\
2510\end{array}$ & $\begin{array}{l}1973-79 \\
1984- \\
1985- \\
1980-84 \\
1939-\end{array}$ \\
\hline $\begin{array}{l}07188 \emptyset 0 \emptyset \\
07188000 \\
0718814 \emptyset \\
07188500 \\
07188500\end{array}$ & $\begin{array}{l}\text { Spring } R \text { nr Quapaw } \\
\text { Spring } R \text { nr Quapaw } \\
\text { Flint Br nr Peoria } \\
\text { Lost Ck at Seneca MO } \\
\text { Lost Ck at Seneca MO }\end{array}$ & $\begin{array}{l}\text { QWP } \\
\text { QWMP } \\
\text { CS } \\
\text { CS } \\
\text { QWP }\end{array}$ & $\begin{array}{l}\mathrm{CP} \\
\mathrm{CNP} \\
\mathrm{CP}\end{array}$ & & $H G$ & $\begin{array}{l}2510 \\
2510 . \\
4.90 \\
42.0 \\
42.0\end{array}$ & $\begin{array}{l}1948-58,60-63 \\
1973-79 \\
1964-85 \\
1949-59 *, 60-74 \\
1951-58\end{array}$ \\
\hline $\begin{array}{l}07189000 \\
\emptyset 7189000 \\
\emptyset 7189000 \\
\emptyset 7189480 \\
\emptyset 7189500\end{array}$ & $\begin{array}{l}\text { Elk R nr Tiff City MO } \\
\text { Elk R nr Tiff City MO } \\
\text { Elk } n \text { r Tiff City MO } \\
\text { Wolf Ck nr Grove } \\
\text { Neosho } R \text { nr Grove }\end{array}$ & $\begin{array}{l}\text { STR } \\
\text { QWP } \\
\text { QWMP } \\
\text { LF } \\
\text { STR }\end{array}$ & $\begin{array}{l}\mathrm{CP} \\
\mathrm{CNP}\end{array}$ & $D C P$ & LGC & $\begin{array}{l}872 \text {. } \\
872 \text {. } \\
872 . \\
7.21 \\
9969\end{array}$ & $\begin{array}{l}1939- \\
1948-49,51-58,60-61 \\
1973-77 \\
1966-72 \\
1925-39\end{array}$ \\
\hline $\begin{array}{l}07189700 \\
\varnothing 7189720 \\
\emptyset 7190000 \\
\emptyset 719000 \emptyset \\
07190500\end{array}$ & $\begin{array}{l}\text { Horse Ck at Afton } \\
\text { Horse Ck Trib nr Afton } \\
\text { Lake } 0^{\prime} \text { The Cherokees at Langley } \\
\text { Lake O' The Cherokees at Langley } \\
\text { Neosho } R \text { nr Langley }\end{array}$ & $\begin{array}{l}\text { CS } \\
\text { CS } \\
\text { RES } \\
\text { QWP } \\
\text { STR }\end{array}$ & $\mathrm{CP}$ & RES & $\begin{array}{l}\text { HG } \\
\text { LG } \\
\text { LG }\end{array}$ & $\begin{array}{l}21.9 \\
.81 \\
10298 . \\
10298 \\
10335\end{array}$ & $\begin{array}{l}1966-85 \\
1966-72 \\
194 \varnothing- \\
1948-49 \\
1939-\end{array}$ \\
\hline $\begin{array}{l}\emptyset 7190500 \\
\emptyset 7190500 \\
\emptyset 7190595 \\
07190595 \\
\emptyset 7190595\end{array}$ & $\begin{array}{l}\text { Neosho R nr Langley } \\
\text { Neosho R nr Langley } \\
\text { Big Cabin Ck nr Welch } \\
\text { Big Cabin Ck nr Welch } \\
\text { Big Cabin Ck nr Welch }\end{array}$ & $\begin{array}{l}\text { QWP } \\
\text { QWMP } \\
\text { QWMP } \\
\text { PR } \\
\text { SED }\end{array}$ & $\begin{array}{l}\text { CP } \\
\text { CNP } \\
C P \\
S\end{array}$ & & & $\begin{array}{r}10335 \\
10335 \\
28.1 \\
28.1 \\
28.1\end{array}$ & $\begin{array}{l}195 \emptyset-59 \\
1973-79 \\
198 \emptyset-83 \\
198 \emptyset-83 \\
198 \emptyset-83\end{array}$ \\
\hline $\begin{array}{l}\emptyset 7190597 \\
\emptyset 7190597 \\
\emptyset 7196597 \\
\emptyset 71966 \varnothing \emptyset \\
\emptyset 719662 \emptyset\end{array}$ & $\begin{array}{l}\text { Big Cabin Ck Trib nr Welch } \\
\text { Big Cabin Ck Trib nr Welch } \\
\text { Big Cabin Ck Trib nr Welch } \\
\text { Big Cabin Ck nr Pyramid Corners } \\
\text { W Fk Big Cabin Ck nr Centralia }\end{array}$ & $\begin{array}{l}\text { QWMP } \\
\text { PR } \\
\text { SED } \\
\text { CS } \\
\text { QWMP }\end{array}$ & $\begin{array}{l}\mathrm{CP} \\
\mathrm{SP}\end{array}$ & & & $\begin{array}{l}71.1 \\
13.1\end{array}$ & $\begin{array}{l}1980-83 \\
1980-83 \\
1980-83 \\
1963-72 * *, 73-79 \\
1980-83\end{array}$ \\
\hline $\begin{array}{l}\emptyset 7190620 \\
67190620 \\
\emptyset 7190625 \\
\emptyset 7190625 \\
\emptyset 7196625\end{array}$ & $\begin{array}{l}\text { W Fk Big Cabin Ck nr Centralia } \\
\text { W Fk Big Cabin Ck nr Centralia } \\
\text { M Fk Big Cabin Ck nr Pyramid Corners } \\
\text { M Fk Big Cabin Ck nr Pyramid Corners } \\
\text { M Fk Big Cabin Ck nr Pyramid Corners }\end{array}$ & $\begin{array}{l}\text { PR } \\
\text { SED } \\
\text { QWMP } \\
\text { PR } \\
\text { SED }\end{array}$ & $\begin{array}{l}\mathrm{S} \\
\mathrm{CP} \\
\mathrm{S}\end{array}$ & & & $\begin{array}{l}13.1 \\
13.1 \\
13.4 \\
13.4 \\
13.4\end{array}$ & $\begin{array}{l}1980-83 \\
198 \emptyset-83 \\
198 \emptyset-83 \\
198 \emptyset-83 \\
198 \emptyset-83\end{array}$ \\
\hline
\end{tabular}

* Continuous streamflow records for this period.

** Some records in intervening years.

*** Some records may have been collected previously. 
Table 2.--Station number listing of current and historical gaging stations maintained by the U.S. Geological Survey

September 90, 1987--Continued

[Symbols defined on last page of report]

\begin{tabular}{|c|c|c|c|c|c|c|c|}
\hline $\begin{array}{l}\text { Station } \\
\text { Number }\end{array}$ & Station & $\begin{array}{l}\text { Type } \\
\text { of } \\
\text { Data }\end{array}$ & $\begin{array}{l}\text { Q.W. } \\
\text { Para- } \\
\text { meters }\end{array}$ & $\begin{array}{l}\text { Stage } \\
\text { Source }\end{array}$ & $\begin{array}{l}\text { Coop- } \\
\text { orator }\end{array}$ & $\begin{array}{c}\text { Drainago } \\
\text { Area } \\
\left(m i l \theta^{2}\right)\end{array}$ & Period of Record \\
\hline $\begin{array}{l}\emptyset 719065 \emptyset \\
6719085 \emptyset \\
6719160 \emptyset \\
\varnothing 719160 \emptyset \\
\varnothing 719100 \emptyset\end{array}$ & $\begin{array}{l}\text { Big Cabin Ck nr Vinita } \\
\text { Little Cabin Ck nr Vinita } \\
\text { Big Cabin Ck nr Big Cabin } \\
\text { Big Cabin Ck nr Big Cabin } \\
\text { Big Cabin Ck nr Big Cabin }\end{array}$ & $\begin{array}{l}\text { QWP } \\
\text { QWP } \\
\text { STR } \\
\text { QWP } \\
\text { QWMP }\end{array}$ & $\begin{array}{l}\mathrm{CP} \\
\mathrm{CP} \\
\mathrm{CP} \\
\mathrm{CNP}\end{array}$ & $D C P$ & LGC & $\begin{array}{l}466 \\
466 \\
466\end{array}$ & $\begin{array}{l}1949-51 \\
1948-51 \\
1947- \\
1951-60,64-71 \\
1975-77\end{array}$ \\
\hline $\begin{array}{l}\sigma 71912 \varnothing \emptyset \\
\varnothing 71912 \varnothing \emptyset \\
6719122 \emptyset \\
\sigma 719122 \varnothing \\
\varnothing 719122 \emptyset\end{array}$ & $\begin{array}{l}\text { Spavinaw Ck nr Row } \\
\text { Spavinaw Ck nr Row } \\
\text { Spavinaw Ck nr Sycamore } \\
\text { Spavinaw Ck nr Sycamore } \\
\text { Spavinaw Ck nr Sycamore }\end{array}$ & $\begin{array}{l}\text { STR } \\
\text { QWP } \\
\text { STR } \\
\text { QWMP } \\
\text { QWP }\end{array}$ & $\begin{array}{l}\mathrm{CP} \\
\text { CNP } \\
\text { CNP }\end{array}$ & & $P G$ & $\begin{array}{l}128 . \\
128 \\
133 \\
133 \\
133\end{array}$ & $\begin{array}{l}1959-62 \\
1959-61 \\
1961- \\
1977 \\
1968,77,80-\end{array}$ \\
\hline $\begin{array}{l}67191223 \\
\emptyset 719126 \emptyset \\
6719135 \emptyset \\
67191355 \\
\varnothing 71914 \varnothing \emptyset\end{array}$ & $\begin{array}{l}\text { Spavinaw Ck nr Jay } \\
\text { Brushy Ck } n r \text { Jay } \\
\text { Salina Ck } n r \text { Salina } \\
\text { Spavinaw Ck nr Spavinaw } \\
\text { Lake Hudson } n r \text { Locust Grove }\end{array}$ & $\begin{array}{l}\text { QWP } \\
\text { CS } \\
\text { QWP } \\
\text { QWP } \\
\text { RESP }\end{array}$ & $\begin{array}{l}\mathrm{CP} \\
\mathrm{CP} \\
\mathrm{CP}\end{array}$ & RES & LG & 11534 & $\begin{array}{l}1958-59,61 \\
1965-72 \\
1948-49,51-53,58-59 \\
1948-51 \\
1964-\end{array}$ \\
\hline 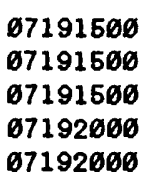 & $\begin{array}{l}\text { Neosho } R \text { nr Chouteau } \\
\text { Neosho } R \text { nr Chouteau } \\
\text { Neosho R nr Chouteau } \\
\text { Pryor Ck nr Pryor } \\
\text { Pryor Ck nr Pryor }\end{array}$ & $\begin{array}{l}\text { STR } \\
\text { QWP } \\
\text { QWMP } \\
\text { STR } \\
\text { QWP }\end{array}$ & $\begin{array}{l}\text { CP } \\
C N P \\
C P\end{array}$ & & LG & $\begin{array}{r}11546 \\
11546 \\
11546 \\
229 \\
229\end{array}$ & $\begin{array}{l}1938-5 \emptyset, 63- \\
1951-58,60 \\
1973-79 \\
1948-64 \\
1948-63\end{array}$ \\
\hline $\begin{array}{l}\varnothing 7192 \varnothing 3 \emptyset \\
\emptyset 719205 \emptyset \\
\emptyset 7192 \varnothing 6 \emptyset \\
671925 \varnothing \emptyset \\
\varnothing 71925 \varnothing \emptyset\end{array}$ & $\begin{array}{l}\text { Pryor Ck at Elliot St Bridge nr Pryor } \\
\text { Pryor Ck at Hwy } 69 \mathrm{~A} \text { Bridge nr Pryor } \\
\text { Pryor Ck blw Sulphur Ck nr Pryor } \\
\text { Neosho R } n r \text { Wagoner } \\
\text { Neosho } R \text { nr Wagoner }\end{array}$ & $\begin{array}{l}\text { QWP } \\
\text { QWP } \\
\text { QWP } \\
\text { STR } \\
\text { QWP }\end{array}$ & $\begin{array}{l}\text { CNP } \\
\text { CP } \\
\text { CNP } \\
C P\end{array}$ & & & $\begin{array}{l}12307 . \\
12307 .\end{array}$ & $\begin{array}{l}1966-71 \\
1958,62-63 \\
1968-75 \\
1924-49 \\
1948-5 \emptyset\end{array}$ \\
\hline $\begin{array}{l}67193000 \\
67193500 \\
6719350 \varnothing \\
6719350 \varnothing \\
6719350 \varnothing\end{array}$ & $\begin{array}{l}\text { Fort Gibson Lake } n r \text { Fort Gibson } \\
\text { Neosho } R \text { blw Ft Gibson } L n r \text { Ft Gibson } \\
\text { Neosho } R \text { blw Ft Gibson } L n r \text { Ft Gibson } \\
\text { Neosho } R \text { blw Ft Gibson } L n r \text { Ft Gibson } \\
\text { Neosho R blw Ft Gibson } L n r \text { Ft Gibson }\end{array}$ & $\begin{array}{l}\text { RES } \\
\text { STGU } \\
\text { QWP } \\
\text { QWMP } \\
\text { SED }\end{array}$ & $\begin{array}{l}\text { BCNP } \\
\text { CNP } \\
S\end{array}$ & $\begin{array}{l}\text { DCP } \\
\text { OBS }\end{array}$ & $\begin{array}{l}C \\
C \\
A\end{array}$ & $\begin{array}{l}12492 \\
12495 \\
12495 \\
12495 \\
12495\end{array}$ & $\begin{array}{l}1949- \\
1950- \\
1952- \\
1973-77 \\
1973-\end{array}$ \\
\hline $\begin{array}{l}\sigma 719450 \emptyset \\
671946 \varnothing \emptyset \\
\sigma 71945 \varnothing \emptyset \\
6719456 \emptyset \\
67194612\end{array}$ & $\begin{array}{l}\text { Arkansas R nr Muskogee } \\
\text { Arkansas } R \text { nr Muskogee } \\
\text { Arkansas R nr Muskogee } \\
\text { Arkansas R nr Muskogee } \\
\text { Bayou Manard nr Ft Gibson }\end{array}$ & $\begin{array}{l}\text { STR } \\
\text { QWP } \\
\text { QWMP } \\
\text { STGU } \\
\text { QWP }\end{array}$ & $\begin{array}{l}\mathrm{CP} \\
\mathrm{CNP} \\
\mathrm{CP}\end{array}$ & BDT & C & $\begin{array}{l}96674 . \\
96674 . \\
96674 \\
96674\end{array}$ & $\begin{array}{l}1926-70 \\
195763 \\
1973-79 \\
1986- \\
1961\end{array}$ \\
\hline $\begin{array}{l}\emptyset 7194515 \\
\varnothing 719454 \emptyset \\
67194545 \\
6719465 \emptyset \\
67194651\end{array}$ & $\begin{array}{l}\text { Mill Ck nr Park Hill } \\
\text { Greenleaf Lake nr Braggs } \\
\text { Greenleaf Ck nr Braggs } \\
\text { Ark } R \text { at Weber Falls L D (US) } \\
\text { Ark } R \text { at Weber Falls L D }\end{array}$ & $\begin{array}{l}\text { CS } \\
\text { QWP } \\
\text { QWP } \\
\text { STGU } \\
\text { QWD }\end{array}$ & $\begin{array}{l}\mathrm{CP} \\
\mathrm{CP} \\
\mathrm{CP}\end{array}$ & DCP & $H G$ & $\begin{array}{l}97049 . \\
97049 .\end{array}$ & $\begin{array}{l}1965-85 \\
1949,51,52 \\
1953-55 \\
1986- \\
1949,57-63\end{array}$ \\
\hline
\end{tabular}

* Continuous streamflow records for this period.

** Some records in intervening years.

*** Some records may have been collected previously. 
Table 2.--Station number listing of current and historical gaging stations maintained by the U.S. Geological Survey

September 30, 1987--Continued

[Symbols defined on last page of report]

\begin{tabular}{|c|c|c|c|c|c|c|c|}
\hline $\begin{array}{l}\text { Station } \\
\text { Number }\end{array}$ & Station Name & $\begin{array}{l}\text { Type } \\
\text { of } \\
\text { Data }\end{array}$ & $\begin{array}{l}\text { Q.W. } \\
\text { Para- } \\
\text { meters }\end{array}$ & $\begin{array}{l}\text { Stage } \\
\text { Source }\end{array}$ & $\begin{array}{l}\text { Coop- } \\
\text { erator }\end{array}$ & $\begin{array}{c}\text { Drainage } \\
\text { Area } \\
\left(m i l e^{2}\right)\end{array}$ & Period of Record \\
\hline $\begin{array}{l}\emptyset 7194551 \\
\emptyset 71955 \emptyset \emptyset \\
\emptyset 71955 \varnothing \emptyset \\
\varnothing 71955 \varnothing \emptyset \\
\emptyset 7196 \varnothing \varnothing \emptyset\end{array}$ & $\begin{array}{l}\text { Ark } R \text { at Weber Falls } L D \\
\text { Illinois } R \mathrm{nr} \text { Watts } \\
\text { Illinois } R \mathrm{nr} \text { Watts } \\
\text { Illinois } R \mathrm{nr} \text { Watts } \\
\text { Flint Ck nr Kansas }\end{array}$ & $\begin{array}{l}\text { STGU } \\
\text { STR } \\
\text { QWP } \\
\text { QWMP } \\
\text { QWP }\end{array}$ & $\begin{array}{l}\text { CNP } \\
\text { CNP } \\
C P\end{array}$ & DCP & $\begin{array}{l}c \\
C\end{array}$ & $\begin{array}{r}97049 . \\
635 \\
635 \\
635 \\
110\end{array}$ & $\begin{array}{l}1986- \\
1955- \\
1955-61,63,69-73 \\
1975-79 \\
1965-61,63\end{array}$ \\
\hline $\begin{array}{l}\emptyset 7196 \emptyset \emptyset \emptyset \\
\emptyset 7196 \varnothing 6 \emptyset \\
\emptyset 7196 \emptyset 1 \emptyset \\
\emptyset 719638 \emptyset \\
\emptyset 71965 \varnothing \emptyset\end{array}$ & $\begin{array}{l}\text { Flint Ck nr Kansas } \\
\text { Flint Ck nr Kansas } \\
\text { Flint Ck Trib nr Flint } \\
\text { Illinois R Trib nr Tahlequah } \\
\text { Illinois R nr Tahlequah }\end{array}$ & $\begin{array}{l}\text { QWMP } \\
\text { STR } \\
\text { CS } \\
\text { CS } \\
\text { STR }\end{array}$ & CNP & DCP & JG & $\begin{array}{l}110 . \\
110 . \\
6.94 \\
3.59 \\
959 .\end{array}$ & $\begin{array}{l}1975-79 \\
1979- \\
1966-72 \\
1965-75 \\
1935-\end{array}$ \\
\hline $\begin{array}{l}\emptyset 71965 \varnothing \emptyset \\
\varnothing 71965 \varnothing \emptyset \\
\varnothing 71965 \varnothing \emptyset \\
\varnothing 719651 \emptyset \\
\varnothing 71969 \varnothing \emptyset\end{array}$ & $\begin{array}{l}\text { Illinois } R \text { nr Tahlequah } \\
\text { Illinois } R \text { nr Tahlequah } \\
\text { Illinois } R \text { nr Tahlequah } \\
\text { Tahlequah Ck at Tahlequah } \\
\text { Baron Fk at Dutch Mills Ark }\end{array}$ & $\begin{array}{l}\text { QWP } \\
\text { QWMP } \\
\text { SED } \\
\text { QWMP } \\
\text { QWP }\end{array}$ & $\begin{array}{l}\text { CP } \\
\text { CNP } \\
S \\
\text { CNP } \\
C\end{array}$ & & C & $\begin{array}{l}959 . \\
959 . \\
959 . \\
13.4 \\
43.0\end{array}$ & $\begin{array}{l}1960-61 \\
1975-79 \\
1976- \\
1976-77 \\
1959-61\end{array}$ \\
\hline $\begin{array}{l}\varnothing 7197 \varnothing \varnothing \varnothing \\
\varnothing 7197 \varnothing \varnothing \varnothing \\
\varnothing 7197 \varnothing \varnothing \emptyset \\
\varnothing 71975 \varnothing \emptyset \\
\varnothing 719752 \varnothing\end{array}$ & $\begin{array}{l}\text { Baron Fk at Eldon } \\
\text { Baron Fk at Eldon } \\
\text { Baron Fk at Eldon } \\
\text { Tenkiller Ferry Lake nr Gore } \\
\text { Illinois } R \text { at Tenkiller L TW }\end{array}$ & $\begin{array}{l}\text { STR } \\
\text { QWP } \\
\text { QWMP } \\
\text { RES } \\
\text { STGU }\end{array}$ & $\begin{array}{l}\text { CP } \\
\text { CNP }\end{array}$ & $\begin{array}{l}\text { DCP } \\
\text { DCP }\end{array}$ & CJG & $\begin{array}{l}367 . \\
367 . \\
367 . \\
1610 .\end{array}$ & $\begin{array}{l}1948- \\
1958-60 \\
1975-79 \\
1952- \\
1986-\end{array}$ \\
\hline $\begin{array}{l}\emptyset 7198 \emptyset \emptyset \emptyset \\
\varnothing 7198 \emptyset \emptyset \emptyset \\
\varnothing 7198 \varnothing \emptyset \emptyset \\
\emptyset 71985 \emptyset \emptyset \\
\emptyset 719850 \emptyset\end{array}$ & $\begin{array}{l}\text { Illinois } R \text { nr Gore } \\
\text { Illinois } R \text { nr Gore } \\
\text { Illinois } R \text { nr Gore } \\
\text { Dirty Ck nr Warner } \\
\text { Dirty Ck nr Warner }\end{array}$ & $\begin{array}{l}\text { STR } \\
\text { QWP } \\
\text { QWMP } \\
\text { STRP } \\
\text { QWP }\end{array}$ & $\begin{array}{l}\text { CP } \\
\text { CNP } \\
C P\end{array}$ & DCP & $\begin{array}{l}C \\
J G\end{array}$ & $\begin{array}{l}1626 \\
1626 \\
1626 \\
227 \\
227\end{array}$ & $\begin{array}{l}1939 * * *, 48,62,54- \\
1948- \\
1973-79 \\
1946-46 \\
1980-61\end{array}$ \\
\hline $\begin{array}{l}\emptyset 71988 \emptyset \emptyset \\
\emptyset 71988 \emptyset \emptyset \\
\varnothing 71988 \emptyset \emptyset \\
\emptyset 7228 \varnothing \emptyset \emptyset \\
\varnothing 722815 \emptyset\end{array}$ & $\begin{array}{l}\text { South Fk } n r \text { Porum } \\
\text { South Fk } n r \text { Porum } \\
\text { South Fk } n r \text { Porum } \\
\text { Canadian } R n r \text { Canadian TX } \\
\text { Commission Ck nr Grand }\end{array}$ & $\begin{array}{l}\text { QWMP } \\
\text { PR } \\
\text { SED } \\
\text { QWMP } \\
\text { LF }\end{array}$ & $\begin{array}{l}C P \\
S \\
C N P\end{array}$ & & & $\begin{array}{r}48.7 \\
48.7 \\
48.7 \\
22866 . \\
67.8\end{array}$ & $\begin{array}{l}198 \emptyset-83 \\
198 \emptyset-83 \\
198 \emptyset-83 \\
1973-77 \\
1965-66\end{array}$ \\
\hline $\begin{array}{l}\emptyset 72282 \emptyset \emptyset \\
\emptyset 72282 \emptyset \emptyset \\
\emptyset 722822 \emptyset \\
\emptyset 722825 \emptyset \\
\emptyset 722828 \emptyset\end{array}$ & $\begin{array}{l}\text { Canadian } R \text { nr Roll } \\
\text { Canadian } R \text { nr Roll } \\
\text { Turkey Ck nr Camargo } \\
\text { Canadian } R \text { at Taloga } \\
\text { Little Robe } C k \text { nr Oakwood }\end{array}$ & $\begin{array}{l}\text { QWP } \\
\text { QWMP } \\
\text { LF } \\
\text { QWP } \\
\text { CS }\end{array}$ & $\begin{array}{l}\text { CP } \\
\text { CNP } \\
C\end{array}$ & & & $\begin{array}{l}23616 . \\
23616 . \\
86 . \emptyset \\
6.3 \emptyset\end{array}$ & $\begin{array}{l}1950-53,62-63 \\
196 \emptyset-61,76-77 \\
1965-73 \\
1953 \\
1966-72\end{array}$ \\
\hline $\begin{array}{l}\emptyset 722829 \emptyset \\
\emptyset 72283 \emptyset \emptyset \\
\emptyset 72284 \emptyset \emptyset \\
\emptyset 72284 \emptyset \emptyset \\
\emptyset 722846 \emptyset\end{array}$ & $\begin{array}{l}\text { Rough } C_{k} n r \text { Thomas } \\
\text { Canadian } R \text { nr Thomas } \\
\text { Deer Ck at Hydro } \\
\text { Deer Ck at Hydro } \\
\text { Deer Ck Trib nr Hydro }\end{array}$ & $\begin{array}{l}\text { CS } \\
\text { QWP } \\
\text { STR } \\
\text { QWP } \\
\text { CS }\end{array}$ & $\begin{array}{l}\mathrm{CP} \\
\mathrm{CNP}\end{array}$ & & HG & $\begin{array}{l}10.4 \\
274 . \\
274 . \\
2.31\end{array}$ & $\begin{array}{l}1964-85 \\
1962-53,61-63 \\
1981-64,77-80 \\
1951-58,60-63,77-80 \\
1964-76\end{array}$ \\
\hline
\end{tabular}

* Continuous streamf low records for this period.

** Some records in intervening years.

*** Some records may have been collected previously. 
Table 2.--Station number listing of current and historical gaging stations maintained by the U.S. Geological Survey September $30,1987--C o n t i n u e d$

[Symbols defined on last page of report]

\begin{tabular}{|c|c|c|c|c|c|c|c|}
\hline $\begin{array}{c}\text { Station } \\
\text { Number }\end{array}$ & Station & $\begin{array}{l}\text { Type } \\
\text { of } \\
\text { Data }\end{array}$ & $\begin{array}{l}\text { Q.W. } \\
\text { Para- } \\
\text { meters }\end{array}$ & $\begin{array}{l}\text { Stage } \\
\text { Source }\end{array}$ & $\begin{array}{l}\text { Coop- } \\
\text { erator }\end{array}$ & $\begin{array}{c}\text { Drainage } \\
\text { Area } \\
\left(\text { mile }{ }^{2}\right)\end{array}$ & Period of Record \\
\hline $\begin{array}{l}\emptyset 72285 \emptyset \emptyset \\
672285 \emptyset \emptyset \\
\emptyset 72285 \emptyset \emptyset \\
\emptyset 72285 \emptyset \emptyset \\
\emptyset 72285 \varnothing \emptyset\end{array}$ & $\begin{array}{l}\text { Canadian } R \text { at Bridgeport } \\
\text { Canadian } R \text { at Bridgeport } \\
\text { Canadian } R \text { at Bridgeport } \\
\text { Canadian } R \text { at Bridgeport } \\
\text { Canadian } R \text { at Bridgeport }\end{array}$ & $\begin{array}{l}\text { STR } \\
\text { QWD } \\
\text { QWMP } \\
\text { SED } \\
\text { QWP }\end{array}$ & $\begin{array}{l}\text { CNP } \\
\text { CNP } \\
\text { S } \\
\text { CNOPS }\end{array}$ & OBS & $J G$ & $\begin{array}{l}25229 \\
25229 \\
25229 \\
25229 \\
25229\end{array}$ & $\begin{array}{l}1944-64,69- \\
1949-61,64,70-81 \\
1973-79 \\
1978-81 \\
1981-\end{array}$ \\
\hline $\begin{array}{l}\emptyset 72286 \varnothing \emptyset \\
672287 \varnothing \emptyset \\
\varnothing 722893 \emptyset \\
\varnothing 722896 \varnothing \\
\varnothing 7229 \varnothing \varnothing \emptyset\end{array}$ & $\begin{array}{l}\text { Canyon View Ck nr Geary } \\
\text { Canadian } R \text { nr Union City } \\
\text { Worley Ck nr Tuttle } \\
\text { Canadian R Trib nr Newcastle } \\
\text { Canadian } R \text { nr Newcastle }\end{array}$ & $\begin{array}{l}\text { CS } \\
\text { QWP } \\
\text { CS } \\
\text { CS } \\
\text { STR }\end{array}$ & CNP & & HG & $\begin{array}{c}11.8 \\
11.2 \\
3.32 \\
25763 .\end{array}$ & $\begin{array}{l}1964-72 \\
1952-53,73 \\
1965-72,75-85 \\
1965-75 \\
1939-45\end{array}$ \\
\hline $\begin{array}{l}\varnothing 722903 \emptyset \\
6722905 \emptyset \\
\emptyset 7229655 \\
67229655 \\
\varnothing 722916 \varnothing\end{array}$ & $\begin{array}{l}\text { Merkle } \mathrm{Ck} \text { at Norman } \\
\text { Canadian } \mathrm{R} \text { at Norman } \\
\text { Bishop } \mathrm{Cr} \text { at Norman } \\
\text { Bishop } \mathrm{Cr} \text { at Norman } \\
\text { Canadian } \mathrm{R} \text { n } \mathrm{r} \text { Noble }\end{array}$ & $\begin{array}{l}\text { CSR } \\
\text { QWP } \\
\text { CSR } \\
\text { CSR } \\
\text { STR }\end{array}$ & CP & & $\begin{array}{l}c \\
c\end{array}$ & 25911 . & $\begin{array}{l}1988- \\
1958 \\
1987- \\
1988- \\
1960-61,63-75\end{array}$ \\
\hline $\begin{array}{l}\varnothing 72291 \varnothing \varnothing \\
\varnothing 72291 \varnothing \emptyset \\
\varnothing 72291 \varnothing \varnothing \\
\varnothing 72292 \varnothing \emptyset \\
\emptyset 72292 \varnothing \emptyset\end{array}$ & $\begin{array}{l}\text { Canadian } R \text { nr Noble } \\
\text { Canadian } R \text { nr Noble } \\
\text { Canadian } R \text { nr Noble } \\
\text { Canadian } R \text { at Purcell } \\
\text { Canadian } R \text { at Purcell }\end{array}$ & $\begin{array}{l}\text { QWD } \\
\text { QWMP } \\
\text { SED } \\
\text { STR } \\
\text { QWD }\end{array}$ & $\begin{array}{l}\text { CNP } \\
\text { CNP } \\
S \\
\text { CNP }\end{array}$ & & JG & $\begin{array}{l}25911 \\
25911 \\
25911 \\
25939 \\
25939\end{array}$ & $\begin{array}{l}1965-71,73-74 \\
1973-75 \\
1976 \\
1960-61,79-82,86- \\
1952, * * 66-63,74-75\end{array}$ \\
\hline $\begin{array}{l}\emptyset 72292 \varnothing \emptyset \\
\varnothing 72292 \varnothing \emptyset \\
\varnothing 722922 \emptyset \\
\emptyset 72293 \varnothing \emptyset \\
\varnothing 72293 \varnothing \emptyset\end{array}$ & $\begin{array}{l}\text { Canadian } R \text { at Purcell } \\
\text { Canadian } R \text { at Purcell } \\
\text { Walnut } C k \text { nr Blanchard } \\
\text { Walnut } C k \text { at Purcell } \\
\text { Walnut } C k \text { at Purcell }\end{array}$ & $\begin{array}{l}\text { QWMP } \\
\text { SED } \\
\text { CS } \\
\text { STR } \\
\text { QWP }\end{array}$ & $\begin{array}{l}\text { CNP } \\
S \\
C P\end{array}$ & & $J G$ & $\begin{array}{l}25939 . \\
25939 . \\
1.26 \\
202 . \\
202\end{array}$ & $\begin{array}{l}1975-79 \\
1979-82 \\
1964-72 \\
1965- \\
1950-65,58-62\end{array}$ \\
\hline $\begin{array}{l}\emptyset 72293 \emptyset \emptyset \\
\emptyset 722942 \emptyset \\
\emptyset 7229425 \\
\emptyset 7229427 \\
\emptyset 7229427\end{array}$ & $\begin{array}{l}\text { Walnut } \mathrm{Ck} \text { at Purcell } \\
\text { Julian } \mathrm{Ck} \text { Trib } \mathrm{nr} \text { Asher } \\
\text { Spring Ck } n r \text { Ada } \\
\text { Canadian Sandy Ck } \mathrm{nr} \text { Ada } \\
\text { Canadian Sandy Ck nr Ada }\end{array}$ & $\begin{array}{l}\text { QWMP } \\
\text { CS } \\
\text { QWP } \\
\text { STR } \\
\text { QWP }\end{array}$ & $\begin{array}{l}\text { CNP } \\
\text { CP } \\
\text { C }\end{array}$ & $N$ & $\begin{array}{l}\mathrm{HG} \\
\mathrm{B} \\
\mathrm{P}\end{array}$ & 2.28 & $\begin{array}{l}1976-77 \\
1964-85 \\
1952-63 \\
1986- \\
1986-\end{array}$ \\
\hline $\begin{array}{l}\emptyset 7229427 \\
\emptyset 722943 \emptyset \\
\emptyset 7229441 \\
\emptyset 7229442 \\
\emptyset 722946 \emptyset\end{array}$ & $\begin{array}{l}\text { Canadian Sandy Ck nr Ada } \\
\text { Arbeca Ck nr Allen } \\
\text { Elm Ck nr Moore } \\
\text { E Elm Ck nr Moore } \\
\text { Little R abv Lake Thunderbird nr Norman }\end{array}$ & $\begin{array}{l}\text { SED } \\
\text { CS } \\
\text { QWP } \\
\text { QWP } \\
\text { QWP }\end{array}$ & $\begin{array}{l}\mathrm{S} \\
\mathrm{CP} \\
\mathrm{CP} \\
\mathrm{BCNP}\end{array}$ & & $B$ & 2.26 & $\begin{array}{l}1987- \\
1964-74 \\
1960-61 \\
196 \emptyset \\
1983-86\end{array}$ \\
\hline $\begin{array}{l}\emptyset 72295 \emptyset \emptyset \\
\emptyset 72295 \emptyset \emptyset \\
\emptyset 72296 \emptyset 1 \\
\emptyset 72298 \emptyset 1 \\
\emptyset 72299 \emptyset \emptyset\end{array}$ & $\begin{array}{l}\text { Little R nr Norman } \\
\text { Little R nr Norman } \\
\text { Clear Ck nr Norman } \\
\text { Hog Ck nr Stella } \\
\text { Lake Thunderbird nr Norman }\end{array}$ & $\begin{array}{l}\text { STR } \\
\text { QWP } \\
\text { QWP } \\
\text { QWP } \\
\text { RESP }\end{array}$ & $\begin{array}{l}C P \\
C P \\
C P\end{array}$ & RES & GM & $\begin{array}{l}120 . \\
120\end{array}$ & $\begin{array}{l}1962-55 \\
1961-57,59,61 \\
1961 \\
1960-61 \\
1965-\end{array}$ \\
\hline
\end{tabular}

* Continuous streamflow records for this period.

** Some records in intervening years.

*** Some records may have been collected previously. 
Table 2.--Station number listing of current and historical gaging stations maintained by the U.S. Geological Survey

September 30, 1987--Continued

[Symbols defined on last page of report]

\begin{tabular}{|c|c|c|c|c|c|c|c|}
\hline $\begin{array}{l}\text { Station } \\
\text { Number }\end{array}$ & Station & $\begin{array}{l}\text { Type } \\
\text { of } \\
\text { Data }\end{array}$ & $\begin{array}{l}\text { Q.W. } \\
\text { Para- } \\
\text { meters }\end{array}$ & $\begin{array}{l}\text { Stage } \\
\text { Source }\end{array}$ & $\begin{array}{l}\text { Coop- } \\
\text { orator }\end{array}$ & $\begin{array}{c}\text { Drainage } \\
\text { Area } \\
\left(m i l e^{2}\right)\end{array}$ & Period of Record \\
\hline 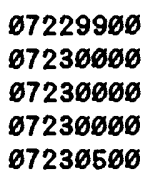 & $\begin{array}{l}\text { Lake Thunderbird nr Norman } \\
\text { Little } R \text { blw Lake Thunderbird } n r \text { Norman } \\
\text { Little } R \text { blw Lake Thunderbird } n r \text { Norman } \\
\text { Little } R \text { blw Lake Thunderbird } n r \text { Norman } \\
\text { Little } R \mathrm{nr} \text { Tecumseh }\end{array}$ & $\begin{array}{l}\text { QWP } \\
\text { STR } \\
\text { QWP } \\
\text { QWMP } \\
\text { STR }\end{array}$ & $\begin{array}{l}\mathrm{CP} \\
\mathrm{CP} \\
\mathrm{CNP}\end{array}$ & & $\begin{array}{l}\text { JGB } \\
\text { GM } \\
\text { C }\end{array}$ & $\begin{array}{l}256 . \\
257 \\
257 \\
257 \\
456\end{array}$ & $\begin{array}{l}1965- \\
1952- \\
1953-65 \\
1975-79 \\
1943-\end{array}$ \\
\hline 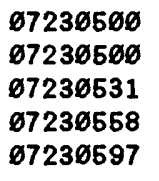 & $\begin{array}{l}\text { Little R nr Tecumseh } \\
\text { Little R } n r \text { Tecumseh } \\
\text { Little R } n r \text { Harjo } \\
\text { Little R } n r \text { Maud } \\
\text { Little R } n r \text { Bowlegs }\end{array}$ & $\begin{array}{l}\text { QWP } \\
\text { SED } \\
\text { QWP } \\
\text { QWP } \\
\text { STR }\end{array}$ & $\begin{array}{l}C P \\
S \\
C P \\
C P\end{array}$ & OBS & B & $\begin{array}{l}456 \\
456\end{array}$ & $\begin{array}{l}1951-63 \\
* * * 1973-78 \\
1961 \\
1959,61 \\
1983-\end{array}$ \\
\hline 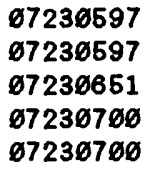 & $\begin{array}{l}\text { Little } R \text { nr Bowlegs } \\
\text { Little R nr Bowlegs } \\
\text { Salt Ck nr Trousdale } \\
\text { Salt Ck nr Pearson } \\
\text { Salt Ck nr Pearson }\end{array}$ & $\begin{array}{l}\text { QWP } \\
\text { SED } \\
\text { QWP } \\
\text { LF } \\
\text { QWP }\end{array}$ & $\begin{array}{l}\text { CNP } \\
S \\
\text { CP } \\
\text { CNP }\end{array}$ & & $\begin{array}{l}\text { B } \\
\text { B }\end{array}$ & $\begin{array}{l}550 . \\
550 . \\
83.8 \\
83.8\end{array}$ & $\begin{array}{l}1953,69,61,83- \\
1983- \\
1961 \\
1955-56 \\
1956-61\end{array}$ \\
\hline 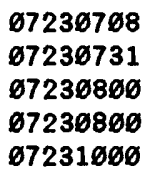 & $\begin{array}{l}\text { Blacksmith Ck nr Pearson } \\
\text { Salt Ck nr St Louis } \\
\text { Salt Ck nr Dewright } \\
\text { Salt Ck nr Dewright } \\
\text { Little R nr Sasakwa }\end{array}$ & $\begin{array}{l}\text { QWP } \\
\text { QWP } \\
\text { STR } \\
\text { QWP } \\
\text { STR }\end{array}$ & $\begin{array}{l}\mathrm{CP} \\
\mathrm{CP} \\
\mathrm{CP}\end{array}$ & & JG & $\begin{array}{l}210 \\
210 \\
865\end{array}$ & $\begin{array}{l}1961 \\
1959-61 \\
1960-63,66-67 \\
1952-63 \\
1942-\end{array}$ \\
\hline $\begin{array}{l}\emptyset 7231 \varnothing \varnothing \emptyset \\
\varnothing 7231 \varnothing \varnothing \emptyset \\
\varnothing 7231 \varnothing \varnothing \emptyset \\
\varnothing 72311 \varnothing 2 \\
\varnothing 723132 \varnothing\end{array}$ & $\begin{array}{l}\text { Little R nr Sasakwa } \\
\text { Little R nr Sasakwa } \\
\text { Little R nr Sasakwa } \\
\text { Deop Ck nr Spaulding } \\
\text { Leader Ck Trib nr Atwood }\end{array}$ & $\begin{array}{l}\text { QWP } \\
\text { QWMP } \\
\text { SED } \\
\text { QWP } \\
\text { CS }\end{array}$ & $\begin{array}{l}C P \\
C N \\
S \\
C P\end{array}$ & & $H G$ & $\begin{array}{l}865 . \\
865 . \\
865 . \\
\quad 6.72\end{array}$ & $\begin{array}{l}1951- \\
1975-79 \\
1976 \\
1961 \\
1964-85\end{array}$ \\
\hline $\begin{array}{l}\emptyset 72315 \emptyset \emptyset \\
\varnothing 72315 \varnothing \emptyset \\
\varnothing 72315 \emptyset \emptyset \\
\emptyset 72315 \emptyset \emptyset \\
\varnothing 723156 \emptyset\end{array}$ & $\begin{array}{l}\text { Canadian } R \text { at Calvin } \\
\text { Canadian } R \text { at Calvin } \\
\text { Canadian } R \text { at Calvin } \\
\text { Canadian } R \text { at Calvin } \\
\text { Middlo } C k \text { n Carson }\end{array}$ & $\begin{array}{l}\text { STR } \\
\text { QWP } \\
\text { QWMP } \\
\text { SED } \\
\text { CS }\end{array}$ & $\begin{array}{l}\text { BCNP } \\
\text { CNP } \\
S\end{array}$ & $D C P$ & $\begin{array}{l}\text { C } \\
\text { JGAE } \\
\text { CAE }\end{array}$ & $\begin{array}{l}27952 . \\
27952 . \\
27952 \text {. } \\
27952 . \\
\quad 7.40\end{array}$ & $\begin{array}{l}* * 1965-\varnothing 6,44- \\
195 \emptyset-53,6 \varnothing-61,65- \\
1973-77 \\
1975-85 \\
1964-74\end{array}$ \\
\hline $\begin{array}{l}\emptyset 723195 \emptyset \\
67231955 \\
\varnothing 7231955 \\
67231962 \\
\varnothing 7231962\end{array}$ & $\begin{array}{l}\text { Pine Ck nr Higgins } \\
\text { Gaines Ck nr Higgins } \\
\text { Gaines Ck nr Higgins } \\
\text { Ti Ck abv unnamed Trib nr Blanco } \\
\text { Ti Ck abv unnamed Trib nr Blanco }\end{array}$ & $\begin{array}{l}\text { CS } \\
\text { PR } \\
\text { QWP } \\
\text { CS } \\
\text { QWP }\end{array}$ & $\begin{array}{l}\text { CNP } \\
\text { CNOP }\end{array}$ & & HG & $\begin{array}{l}1.83 \\
1.83\end{array}$ & $\begin{array}{l}1964-86 \\
1978-8 \emptyset \\
1978-8 \emptyset \\
198 \emptyset-81 \\
198 \emptyset-81\end{array}$ \\
\hline $\begin{array}{l}\emptyset 7231965 \\
67231965 \\
67231985 \\
\emptyset 7231975 \\
67231975\end{array}$ & $\begin{array}{l}\text { Ti Ck nr Blanco } \\
\text { Ti Ck nr Blanco } \\
\text { Ti Ck nr Blanco } \\
\text { Brushy Ck nr Haileyville } \\
\text { Brushy Ck nr Haileyville }\end{array}$ & $\begin{array}{l}\text { STR } \\
\text { QWP } \\
\text { SED } \\
\text { STR } \\
\text { QWP }\end{array}$ & $\begin{array}{l}\text { BCNP } \\
S \\
\text { BCNP }\end{array}$ & & & $\begin{array}{l}4.82 \\
4.82 \\
4.82 \\
139 . \\
139 .\end{array}$ & $\begin{array}{l}198 \emptyset-81 \\
198 \emptyset-81 \\
198 \emptyset-81 \\
1978-82 \\
1978-82\end{array}$ \\
\hline
\end{tabular}

* Continuous streamflow records for this period.

* Some records in intervening years.

*** Some records may have been collected proviously. 
Table 2.--Station number listing of current and historical gaging stations maintained by the U.S. Geological Survey

September so, 1987--Continued

[Symbols defined on last page of report]

\begin{tabular}{|c|c|c|c|c|c|c|c|}
\hline $\begin{array}{c}\text { Station } \\
\text { Number }\end{array}$ & Station & $\begin{array}{l}\text { Type } \\
\text { of } \\
\text { Data }\end{array}$ & $\begin{array}{l}\text { Q.W. } \\
\text { Para- } \\
\text { meters }\end{array}$ & $\begin{array}{l}\text { Stage } \\
\text { Source }\end{array}$ & $\begin{array}{l}\text { Coop- } \\
\text { orator }\end{array}$ & $\begin{array}{c}\text { Drainage } \\
\text { Area } \\
\left(m i l e^{2}\right)\end{array}$ & Period of Record \\
\hline $\begin{array}{l}\emptyset 7231976 \\
\varnothing 723199 \emptyset \\
\varnothing 723199 \emptyset \\
\varnothing 723199 \emptyset \\
\varnothing 7232 \varnothing \varnothing \emptyset\end{array}$ & $\begin{array}{l}\text { Brushy Ck nr Haileyville } \\
\text { Peaceable Ck nr Haileyville } \\
\text { Peaceable Ck nr Haileyville } \\
\text { Peaceable Ck nr Haileyville } \\
\text { Gaines Ck nr Krebs }\end{array}$ & $\begin{array}{l}\text { SED } \\
\text { STR } \\
\text { QWP } \\
\text { SED } \\
\text { STRP }\end{array}$ & $\begin{array}{l}S \\
\text { BCNP } \\
S\end{array}$ & & & $\begin{array}{l}139 . \\
134 \\
134 \\
134 \\
588\end{array}$ & $\begin{array}{l}1978-81 \\
1978-82 \\
1978-82 \\
1978-82 \\
1943-63\end{array}$ \\
\hline $\begin{array}{l}67232000 \\
67232068 \\
67232008 \\
67232068 \\
\varnothing 7232009\end{array}$ & $\begin{array}{l}\text { Gaines Ck nr Krebs } \\
\text { Blue Ck Trib A nr Blocker } \\
\text { Blue Ck Trib A nr Blocker } \\
\text { Blue Ck Trib A nr Blocker } \\
\text { Blue Ck Trib B nr Blocker }\end{array}$ & $\begin{array}{l}\text { QWP } \\
\text { PR } \\
\text { QWP } \\
\text { SED } \\
\text { PR }\end{array}$ & $\begin{array}{l}\mathrm{CP} \\
\mathrm{CNP} \\
\mathrm{S}\end{array}$ & & & $\begin{array}{r}588 . \\
4.67 \\
4.67 \\
4.67 \\
0.22\end{array}$ & $\begin{array}{l}1946-47,50-51,6 \varnothing-62 \\
1978-81 \\
1978-81 \\
1978-81 \\
1976-78\end{array}$ \\
\hline $\begin{array}{l}\emptyset 7232 \varnothing \varnothing 9 \\
\varnothing 7232 \varnothing \varnothing 9 \\
\varnothing 7232 \varnothing 1 \varnothing \\
\varnothing 7232 \varnothing 1 \emptyset \\
\varnothing 7232 \varnothing 1 \varnothing\end{array}$ & $\begin{array}{l}\text { Blue Ck Trib B nr Blocker } \\
\text { Blue Ck Trib B nr Blocker } \\
\text { Blue Ck nr Blocker } \\
\text { Blue Ck nr Blocker } \\
\text { Blue Ck nr Blocker }\end{array}$ & $\begin{array}{l}\text { QWP } \\
\text { SED } \\
\text { STR } \\
\text { QWP } \\
\text { SED }\end{array}$ & $\begin{array}{l}\text { CNP } \\
S \\
\text { CNP } \\
S\end{array}$ & & & $\begin{array}{l}0.22 \\
0.22 \\
12.1 \\
12.1 \\
12.1\end{array}$ & $\begin{array}{l}1976-78 \\
1976-78 \\
1976-82 \\
1976-82 \\
1976-81\end{array}$ \\
\hline $\begin{array}{l}97232024 \\
\emptyset 7232024 \\
\emptyset 7232024 \\
\emptyset 7232027 \\
\varnothing 7232029\end{array}$ & $\begin{array}{l}\text { Deer Ck nr MCAlester } \\
\text { Deer Ck nr McAlester } \\
\text { Deer Ck nr McAlester } \\
\text { Coal Ck nr McAlester } \\
\text { Mathuldy Ck nr Crowder }\end{array}$ & $\begin{array}{l}\text { STR } \\
\text { QWP } \\
\text { SED } \\
\text { QWP } \\
\text { PR }\end{array}$ & $\begin{array}{l}\text { CNP } \\
S \\
C P\end{array}$ & & & $\begin{array}{c}38.3 \\
38.3 \\
38.3 \\
196 . \\
5.41\end{array}$ & $\begin{array}{l}1978-80 \\
1978-80 \\
1978-80 \\
1960-61 \\
1976-81\end{array}$ \\
\hline $\begin{array}{l}\emptyset 7232 \varnothing 29 \\
\varnothing 7232 \varnothing 29 \\
\varnothing 7232 \varnothing 31 \\
\varnothing 7232 \varnothing 5 \emptyset \\
\varnothing 7232246\end{array}$ & $\begin{array}{l}\text { Mathuldy Ck nr Crowder } \\
\text { Mathuldy Ck nr Crowder } \\
\text { Rock Ck nr Crowder } \\
\text { Gaines Ck nr Canadian } \\
\text { Beaver R at Felt }\end{array}$ & $\begin{array}{l}\text { QWP } \\
\text { SED } \\
\text { QWP } \\
\text { QWP } \\
\text { QWMP }\end{array}$ & $\begin{array}{l}\text { CNP } \\
S \\
\text { CNP } \\
\text { CP } \\
\text { CNP }\end{array}$ & & & $\begin{array}{r}5.41 \\
5.41 \\
850 .\end{array}$ & $\begin{array}{l}1976-82 \\
1976-81 \\
1961 \\
1960-62 \\
1977\end{array}$ \\
\hline $\begin{array}{l}\emptyset 723226 \emptyset \\
\varnothing 723225 \emptyset \\
\varnothing 72324 \varnothing \emptyset \\
\varnothing 723246 \emptyset \\
\varnothing 72325 \varnothing \emptyset\end{array}$ & $\begin{array}{l}\text { Beaver R nr Felt } \\
\text { Beaver R nr Felt } \\
\text { Beaver (Sand) Ck nr Texhoma } \\
\text { Tepee Ck nr Eva } \\
\text { Beaver R nr Guymon }\end{array}$ & $\begin{array}{l}\text { CS } \\
\text { STR } \\
\text { LF } \\
\text { LF } \\
\text { STR }\end{array}$ & & DCP & JG & $\begin{array}{l}879 . \\
879 . \\
217 \\
95 . \\
2139\end{array}$ & $\begin{array}{l}1972-79 \\
1979- \\
1967-71 \\
1967-73 \\
1937-\end{array}$ \\
\hline $\begin{array}{l}\emptyset 72325 \varnothing \emptyset \\
\varnothing 72325 \varnothing \emptyset \\
\varnothing 72325 \varnothing \emptyset \\
\varnothing 723256 \varnothing \\
\varnothing 723258 \emptyset\end{array}$ & $\begin{array}{l}\text { Beaver } R n r \text { Guymon } \\
\text { Beaver } R n r \text { Guymon } \\
\text { Beaver } R n r \text { Guymon } \\
\text { South Fk Trib nr Guymon } \\
\text { Goff Ck } n r \text { Hough }\end{array}$ & $\begin{array}{l}\text { QWD } \\
\text { QWMP } \\
\text { SED } \\
\text { CS } \\
\text { LF }\end{array}$ & $\begin{array}{l}\text { BCNP } \\
\text { CNP } \\
S \\
S\end{array}$ & & $\begin{array}{l}\mathrm{C} \\
\mathrm{C}\end{array}$ & $\begin{array}{l}2139 . \\
2139 \\
2139 \\
\text { ø. } \\
470\end{array}$ & $\begin{array}{l}1962,64-63,68-79 \\
1973-77 \\
1975- \\
1975- \\
1968-71\end{array}$ \\
\hline $\begin{array}{l}\varnothing 723259 \emptyset \\
\varnothing 723261 \emptyset \\
\varnothing 723262 \varnothing \\
\varnothing 723263 \emptyset \\
\varnothing 723263 \emptyset\end{array}$ & $\begin{array}{l}\text { Goff Ck nr Guymon } \\
\text { Beaver } R n r \text { Optima } \\
\text { Pony Ck nr Optima } \\
\text { Beaver } R n r \text { Hooker } \\
\text { Beaver } R n r \text { Hooker }\end{array}$ & $\begin{array}{l}\text { LF } \\
\text { QWP } \\
\text { LF } \\
\text { QWP } \\
\text { QWMP }\end{array}$ & $\begin{array}{l}\mathrm{CP} \\
\mathrm{CP} \\
\mathrm{CNP}\end{array}$ & & & $\begin{array}{l}510 . \\
223 \text {. } \\
3017 \\
3617\end{array}$ & $\begin{array}{l}1964-68 \\
1972-73 \\
1966-71 \\
1972-73,76-79 \\
1977\end{array}$ \\
\hline
\end{tabular}

* Continuous streamflow records for this period.

** Some records in intervening years.

*** Some records may have been collected previously. 
Table 2.--Station number listing of current and historical gaging stations maintained by the U.S. Geological Survey

September 30, 1987--Continued

[Symbols defined on last page of report]

\begin{tabular}{|c|c|c|c|c|c|c|c|}
\hline $\begin{array}{l}\text { Station } \\
\text { Number }\end{array}$ & Station & $\begin{array}{l}\text { Typo } \\
\text { of } \\
\text { Data }\end{array}$ & $\begin{array}{l}\text { Q.W. } \\
\text { Para- } \\
\text { meters }\end{array}$ & $\begin{array}{l}\text { Stage } \\
\text { Source }\end{array}$ & $\begin{array}{l}\text { Coop- } \\
\text { erator }\end{array}$ & $\begin{array}{c}\text { Drainage } \\
\text { Area } \\
\left(\mathrm{mil} \mathrm{e}^{2}\right)\end{array}$ & Period of Record \\
\hline $\begin{array}{l}\emptyset 723266 \emptyset \\
\varnothing 72328 \emptyset \emptyset \\
\varnothing 723295 \emptyset \\
\varnothing 7233 \varnothing \varnothing \emptyset \\
\varnothing 7233 \varnothing \varnothing \emptyset\end{array}$ & $\begin{array}{l}\text { Aqua Frio Ck nr Felt } \\
\text { Panhandle Univ. - NADP at Goodwell } \\
\text { Coldwater Ck nr Guymon } \\
\text { Coldwater Ck nr Hardesty } \\
\text { Coldwater Ck nr Hardesty }\end{array}$ & $\begin{array}{l}\text { CSR } \\
\text { QWH } \\
\text { STR } \\
\text { STR } \\
\text { QWP }\end{array}$ & $\mathrm{CP}$ & OBS & $\begin{array}{l}A \\
\text { GJ }\end{array}$ & $\begin{array}{l}31.0 \\
1967 \\
1967\end{array}$ & $\begin{array}{l}1964-75 \\
1985- \\
1981- \\
1939-64 \\
1952-63\end{array}$ \\
\hline $\begin{array}{l}\emptyset 72332 \emptyset \emptyset \\
\varnothing 723321 \emptyset \\
\varnothing 723321 \emptyset \\
\varnothing 72337 \varnothing \emptyset \\
\varnothing 72337 \varnothing \varnothing\end{array}$ & $\begin{array}{l}\text { Optima Lake } n r \text { Hardesty } \\
\text { Beaver R nr Hardesty } \\
\text { Beaver R nr Hardesty } \\
\text { Palo Duro Ck nr Range } \\
\text { Palo Duro Ck nr Range }\end{array}$ & $\begin{array}{l}\text { RES } \\
\text { STR } \\
\text { SED } \\
\text { LF } \\
\text { QWP }\end{array}$ & $\begin{array}{l}S \\
\text { CNP }\end{array}$ & & $\begin{array}{l}c \\
c \\
c\end{array}$ & $\begin{array}{l}5029 \\
4770 \\
4770 \\
1745 \\
1745\end{array}$ & $\begin{array}{l}1978- \\
1978- \\
1978- \\
1951-73 \\
1952-62\end{array}$ \\
\hline $\begin{array}{l}\emptyset 723385 \emptyset \\
\varnothing 7234 \varnothing \emptyset \emptyset \\
\varnothing 7234 \varnothing \emptyset \emptyset \\
\varnothing 7234 \varnothing \emptyset \emptyset \\
\varnothing 7234 \varnothing \varnothing \emptyset\end{array}$ & $\begin{array}{l}\text { Sharp Ck Trib nr Turpin } \\
\text { Beaver } R \text { at Beaver } \\
\text { Beaver } R \text { at Beaver } \\
\text { Beaver } R \text { at Beaver } \\
\text { Beaver } R \text { at Beaver }\end{array}$ & $\begin{array}{l}\text { CSR } \\
\text { STR } \\
\text { QWD } \\
\text { QWMP } \\
\text { SED }\end{array}$ & $\begin{array}{l}\text { BCNP } \\
\text { CNP } \\
S\end{array}$ & DCP & $A$ & $\begin{array}{l}1.00 \\
7955 \\
7955 \\
7955 . \\
7955\end{array}$ & $\begin{array}{l}1964-75 \\
1937- \\
1952,58-59,62-82 \\
1973-77 \\
1974-\end{array}$ \\
\hline $\begin{array}{l}\emptyset 7234 \varnothing \emptyset \emptyset \\
\varnothing 7234 \varnothing 5 \emptyset \\
\varnothing 72341 \varnothing \emptyset \\
\varnothing 72341 \varnothing \emptyset \\
\varnothing 723413 \emptyset\end{array}$ & $\begin{array}{l}\text { Beaver R at Beaver } \\
\text { N Fk Clear Ck Trib nr Balko } \\
\text { Clear Ck nr Elmwood } \\
\text { Clear Ck nr Elmwood } \\
\text { Duck Pond Ck nr Clear Lake }\end{array}$ & $\begin{array}{l}\text { QWP } \\
\text { CS } \\
\text { STR } \\
\text { QWP } \\
\text { LF }\end{array}$ & $\begin{array}{l}\mathrm{BCNP} \\
\mathrm{CP}\end{array}$ & & $\begin{array}{l}A \\
H G \\
J G\end{array}$ & $\begin{array}{c}7956 . \\
4.22 \\
170 . \\
170 . \\
97.0\end{array}$ & $\begin{array}{l}1983- \\
1964-85 \\
1965- \\
1952-53 \\
1966-73\end{array}$ \\
\hline $\begin{array}{l}\emptyset 72342 \emptyset \emptyset \\
\varnothing 72342 \varnothing \emptyset \\
\varnothing 723425 \emptyset \\
\varnothing 723429 \emptyset \\
\varnothing 72343 \varnothing \emptyset\end{array}$ & $\begin{array}{l}\text { Kiowa Ck nr Slapout } \\
\text { Kiowa Ck nr Slapout } \\
\text { Kiowa Ck Trib nr Laverne } \\
\text { Clear Ck Trib nr Catesby } \\
\text { Clear Ck nr May }\end{array}$ & $\begin{array}{l}\text { LF } \\
\text { QWP } \\
\text { CS } \\
\text { CS } \\
\text { QWP }\end{array}$ & $\begin{array}{l}\mathrm{CP} \\
\mathrm{CP}\end{array}$ & & $H G$ & $\begin{array}{l}371 . \\
371 . \\
2.14 \\
8.51 \\
109 .\end{array}$ & $\begin{array}{l}1945,49-61,63-73 \\
1952-58,60 \\
1964-72 \\
1966-85 \\
1954-58,6 \varnothing\end{array}$ \\
\hline $\begin{array}{l}\emptyset 72345 \varnothing \emptyset \\
\varnothing 72355 \varnothing \emptyset \\
\varnothing 72357 \varnothing \emptyset \\
\varnothing 7236 \varnothing \varnothing \emptyset \\
\varnothing 7236 \varnothing \varnothing \emptyset\end{array}$ & $\begin{array}{l}\text { Beaver R nr Fort Supply } \\
\text { Wolf Ck nr Shattuck } \\
\text { Little Wolf Ck Trib nr Gage } \\
\text { Wolf Ck nr Fargo } \\
\text { Wolf Ck nr Fargo }\end{array}$ & $\begin{array}{l}\text { STGU } \\
\text { STRP } \\
\text { CS } \\
\text { STR } \\
\text { QWP }\end{array}$ & $\mathrm{CP}$ & & & $\begin{array}{l}9615 \\
1183 . \\
18.4 \\
1624 \\
1624\end{array}$ & $\begin{array}{l}* 1937-51,61-79 \\
1938-46 \\
1964-74 \\
1942-76 \\
1958,6 \varnothing-63\end{array}$ \\
\hline $\begin{array}{l}\varnothing 7236 \varnothing \varnothing \emptyset \\
\varnothing 7236 \varnothing 5 \emptyset \\
\varnothing 72365 \varnothing \emptyset \\
\varnothing 72365 \varnothing \emptyset \\
\varnothing 7237 \varnothing \varnothing \emptyset\end{array}$ & $\begin{array}{l}\text { Wolf Ck nr Fargo } \\
\text { Wolf Ck Trib nr Tangier } \\
\text { Fort Supply Lake nr Fort Supply } \\
\text { Fort Supply Lake nr Fort Supply } \\
\text { Wolf Ck nr Fort Supply }\end{array}$ & $\begin{array}{l}\text { SED } \\
\text { CS } \\
\text { RES } \\
\text { QWP } \\
\text { STR }\end{array}$ & $C P$ & $\begin{array}{l}\text { DCP } \\
\text { OBS }\end{array}$ & $\begin{array}{l}c \\
c\end{array}$ & $\begin{array}{l}1624 . \\
6.23 \\
1735 . \\
1735 \\
1739 .\end{array}$ & $\begin{array}{l}* * * 1973-76 \\
1964-72 \\
1942- \\
1949,56,53 \\
1937-\end{array}$ \\
\hline $\begin{array}{l}\varnothing 7237 \varnothing \varnothing \varnothing \\
\varnothing 7237 \varnothing \varnothing \varnothing \\
\varnothing 72375 \varnothing \varnothing \\
\varnothing 72375 \varnothing \emptyset \\
\varnothing 72375 \varnothing \varnothing\end{array}$ & $\begin{array}{l}\text { Wolf Ck nr Fort Supply } \\
\text { Wolf Ck nr Fort Supply } \\
N \text { Canadian } R \text { at Woodward } \\
N \text { Canadian } R \text { at Woodward } \\
N \text { Canadian } R \text { at Woodward }\end{array}$ & $\begin{array}{l}\text { QWP } \\
\text { SED } \\
\text { STR } \\
\text { QWP } \\
\text { QWMP }\end{array}$ & $\begin{array}{l}\text { CP } \\
\mathrm{S} \\
\text { BCNP } \\
\text { CNP }\end{array}$ & DCP & $\begin{array}{l}\text { C } \\
\text { A }\end{array}$ & $\begin{array}{l}1739 \\
1739 \\
11589 \\
11589 \\
11589\end{array}$ & $\begin{array}{l}1951-58,60 \\
1976 \\
1938- \\
* 1955,61-63,75- \\
1973-77\end{array}$ \\
\hline
\end{tabular}

* Continuous streamflow records for this period.

** Some records in intervening years.

*** Some records may have been collected previously. 
Table 2.--Station number listing of current and historical gaging stations maintained by the U.S. Geological Survey

September 30, 1987--Continued

[Symbols defined on last page of report]

\begin{tabular}{|c|c|c|c|c|c|c|c|}
\hline $\begin{array}{l}\text { Station } \\
\text { Number }\end{array}$ & Station & $\begin{array}{l}\text { Type } \\
\text { of } \\
\text { Data }\end{array}$ & $\begin{array}{l}\text { Q.W. } \\
\text { Para- } \\
\text { meters }\end{array}$ & $\begin{array}{l}\text { Stage } \\
\text { Source }\end{array}$ & $\begin{array}{l}\text { Coop- } \\
\text { erator }\end{array}$ & $\begin{array}{c}\text { Drainage } \\
\text { Area } \\
\left(m i l e{ }^{2}\right)\end{array}$ & Period of Record \\
\hline $\begin{array}{l}67237500 \\
6723759 \theta \\
672377 \varnothing \varnothing \\
672377 \varnothing \varnothing \\
6723776 \varnothing\end{array}$ & $\begin{array}{l}\mathrm{N} \text { Canadian } R \text { at Woodward } \\
\text { Indian Ck } n r \text { Woodward } \\
\text { Persimmon Ck nr Mutual } \\
\text { Persimmon Ck nr Mutual } \\
\text { Cottonwood Ck nr Vici }\end{array}$ & $\begin{array}{l}\text { SED } \\
\text { QWP } \\
\text { LF } \\
\text { QWP } \\
\text { CS }\end{array}$ & $\begin{array}{l}S \\
C P \\
C P\end{array}$ & & HG & $\begin{array}{r}11689 . \\
164 \\
164 \\
11.5\end{array}$ & $\begin{array}{l}1975- \\
1951-57 \\
1958-61,65-73 \\
1951,58 \\
1964-85\end{array}$ \\
\hline $\begin{array}{l}\emptyset 723780 \emptyset \\
6723780 \emptyset \\
\varnothing 723780 \emptyset \\
\emptyset 723800 \emptyset \\
\varnothing 723800 \emptyset\end{array}$ & $\begin{array}{l}\text { Bent Ck nr Seiling } \\
\text { Bent Ck nr Seiling } \\
\text { Bent Ck nr Seiling } \\
N \text { Canadian R nr Seiling } \\
N \text { Canadian R nr Seiling }\end{array}$ & $\begin{array}{l}\text { CS } \\
\text { QWP } \\
\text { STR } \\
\text { STR } \\
\text { QWP }\end{array}$ & $\begin{array}{l}\text { CP } \\
\text { BCNPS }\end{array}$ & DCP & $\begin{array}{l}J G \\
G J \\
C\end{array}$ & $\begin{array}{r}139 . \\
139 . \\
139 . \\
12261 \\
12261\end{array}$ & $\begin{array}{l}* 1966-76,71- \\
1956 \\
1966-76 \\
1946- \\
1953-59, *, 74-75\end{array}$ \\
\hline 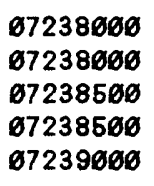 & $\begin{array}{l}N \text { Canadian } R n r \text { Seiling } \\
N \text { Canadian } R n r \text { Seiling } \\
\text { Canton Lake } n r \text { Canton } \\
\text { Canton Lake } n r \text { Canton } \\
N \text { Canadian } R \text { at Canton }\end{array}$ & $\begin{array}{l}\text { QWMP } \\
\text { SED } \\
\text { RES } \\
\text { QWP } \\
\text { STR }\end{array}$ & $\begin{array}{l}\text { CNP } \\
\mathrm{S} \\
\mathrm{CP}\end{array}$ & $\begin{array}{l}D C P \\
D C P\end{array}$ & $\begin{array}{l}C \\
C \\
j G \\
C\end{array}$ & $\begin{array}{l}12261 \\
12261 \\
12483 \\
12483 \\
12484\end{array}$ & $\begin{array}{l}1973-79 \\
* * * 1973- \\
1948- \\
* * 1949-6 \emptyset, 68- \\
1937-\end{array}$ \\
\hline $\begin{array}{l}\varnothing 7239000 \\
\varnothing 7239000 \\
\varnothing 7239000 \\
\varnothing 7239050 \\
\varnothing 7239200\end{array}$ & $\begin{array}{l}N \text { Canadian } R \text { at Canton } \\
N \text { Canadian } R \text { at Canton } \\
N \text { Canadian } R \text { at Canton } \\
N \text { Canadian } R \text { Trib nr Eagle City } \\
N \text { Canadian } R \text { nr Watonga }\end{array}$ & $\begin{array}{l}\text { QWD } \\
\text { QWMP } \\
\text { SED } \\
\text { CS } \\
\text { STR }\end{array}$ & $\begin{array}{l}\text { CP } \\
\text { CNP } \\
S\end{array}$ & & C & $\begin{array}{l}12484 \\
12484 \\
12484 \\
\quad 0.52 \\
12692\end{array}$ & $\begin{array}{l}1951-69 \\
1973-79 \\
1976 \\
1984-75 \\
1954-83\end{array}$ \\
\hline $\begin{array}{l}\emptyset 72392 \varnothing \emptyset \\
6723936 \emptyset \\
\varnothing 723945 \emptyset \\
\emptyset 723945 \emptyset \\
\varnothing 72395 \varnothing \emptyset\end{array}$ & $\begin{array}{l}N \text { Canadian } R \text { nr Watonga } \\
N \text { Canadian } R \text { blw Weavers Ck nr Watonga } \\
N \text { Canadian } R n r \text { Ft Reno } \\
N \text { Canadian } R n r \text { Ft Reno } \\
N \text { Canadian } R n r \text { El Reno }\end{array}$ & $\begin{array}{l}\text { SED } \\
\text { STR } \\
\text { QWP } \\
\text { SED } \\
\text { STR }\end{array}$ & $\begin{array}{l}S \\
\text { BCNP } \\
S\end{array}$ & BDT & KG & 12692 & $\begin{array}{l}* * * 1973-78 \\
1984- \\
1974-75 \\
1974-75 \\
* * 1902-08,37-\end{array}$ \\
\hline $\begin{array}{l}\emptyset 72395 \varnothing \emptyset \\
\varnothing 72395 \varnothing \emptyset \\
\emptyset 72395 \varnothing \emptyset \\
\emptyset 723965 \emptyset \\
\emptyset 72397 \varnothing \emptyset\end{array}$ & $\begin{array}{l}N \text { Canadian } R \text { nr El Reno } \\
N \text { Canadian } R \text { nr El Reno } \\
N \text { Canadian } R n r \text { El Reno } \\
\text { Canadian } R \text { at Cemetary Rd nr Yukon } \\
N \text { Canadian } R \text { nr Yukon }\end{array}$ & $\begin{array}{l}\text { QWD } \\
\text { QWMP } \\
\text { SED } \\
\text { QWP } \\
\text { PR }\end{array}$ & $\begin{array}{l}\text { CP } \\
\text { CNP } \\
S \\
\text { CNOP }\end{array}$ & & KG & $\begin{array}{l}13042 \\
13042 \\
13042 \\
13183\end{array}$ & $\begin{array}{l}* * 1960-51,53,74-75 \\
1973-79 \\
* * * 1973-78 \\
1973 \\
1943\end{array}$ \\
\hline 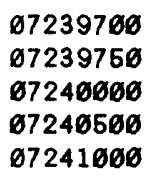 & $\begin{array}{l}N \text { Canadian } R \text { nr Yukon } \\
N \text { Canadian } R \text { at Morgan Rd nr Yukon } \\
\text { Lake Hefner Canal nr OKC } \\
\text { Lake Overholser nr OKC } \\
N \text { Canadian } R \text { blw Lake Ovrholsr nr OKC }\end{array}$ & $\begin{array}{l}\text { QWP } \\
\text { QWP } \\
\text { STR } \\
\text { RESP } \\
\text { STR }\end{array}$ & $\begin{array}{l}\mathrm{CP} \\
\mathrm{CNOP}\end{array}$ & RES & $\begin{array}{l}K G \\
K G \\
K G\end{array}$ & $\begin{array}{l}13221 \\
13222\end{array}$ & $\begin{array}{l}1952-54 \\
1973 \\
1944- \\
1937- \\
* * 1952-68,73-\end{array}$ \\
\hline $\begin{array}{l}\emptyset 724100 \emptyset \\
\emptyset 7241 \emptyset \emptyset \emptyset \\
\emptyset 7241 \emptyset 3 \emptyset \\
\emptyset 7241 \emptyset 31 \\
\emptyset 724108 \emptyset\end{array}$ & $\begin{array}{l}N \text { Canadian } R \text { blw Lake Ovrholsr nr OKC } \\
N \text { Canadian } R \text { blw Lake Ovrholsr nr OKC } \\
S \text { Br T2 Mst'g Ck at SW74, Mustang } \\
S \text { Br T,T2 Mst'g Ck at SW74, Mustang } \\
N \text { Canadian R Trib at NWIg, OKC }\end{array}$ & $\begin{array}{l}\text { QWD } \\
\text { SED } \\
\text { CSR } \\
\text { CSR } \\
\text { CS }\end{array}$ & $\begin{array}{l}C P \\
S\end{array}$ & $D C P$ & C & $\begin{array}{r}13222 . \\
13222 . \\
0.54 \\
\emptyset .29 \\
1.78\end{array}$ & $\begin{array}{l}1968-61 \\
1978- \\
1976-81 \\
1976-81 \\
1976-81\end{array}$ \\
\hline
\end{tabular}

* Continuous streamf low records for this period.

** Some records in intervening years.

*** Some records may have been collected previously. 
Table 2.--Station number listing of current and historical gaging stations maintained by the U.S. Geological Survey

September 90, 1987--Continued

[Symbols defined on last page of report]

\begin{tabular}{|c|c|c|c|c|c|c|c|}
\hline $\begin{array}{l}\text { Station } \\
\text { Number }\end{array}$ & Station & $\begin{array}{l}\text { Type } \\
\text { of } \\
\text { Data }\end{array}$ & $\begin{array}{l}\text { Q.W. } \\
\text { Para- } \\
\text { meters }\end{array}$ & $\begin{array}{l}\text { Stage } \\
\text { Source }\end{array}$ & $\begin{array}{l}\text { Coop- } \\
\text { orator }\end{array}$ & $\begin{array}{c}\text { Drainage } \\
\text { Area } \\
(m i l e)\end{array}$ & Period of Record \\
\hline $\begin{array}{l}\sigma 724121 \emptyset \\
\sigma 724122 \emptyset \\
\sigma 724123 \emptyset \\
\sigma 72415 \sigma \emptyset \\
\sigma 72415 \varnothing \emptyset\end{array}$ & $\begin{array}{l}\text { Twin Ck at SW29, OKC } \\
\text { Brock Ck at SW59, OKC } \\
\text { Lightning Ck at SW74 OKC } \\
N \text { Canadian R nr OKC } \\
N \text { Canadian R nr OKC }\end{array}$ & $\begin{array}{l}\text { CSR } \\
\text { CSR } \\
\text { CS } \\
\text { STR } \\
\text { QWP }\end{array}$ & $C P$ & & & $\begin{array}{l}3.35 \\
2.26 \\
3.96 \\
13354 . \\
13354 .\end{array}$ & $\begin{array}{l}1976-81 \\
1976-81 \\
1976-81 \\
1939-61 \\
1952,60-63\end{array}$ \\
\hline $\begin{array}{l}\emptyset 72415 \emptyset 8 \\
\emptyset 724155 \emptyset \\
\sigma 724155 \emptyset \\
\sigma 724155 \emptyset \\
6724155 \emptyset\end{array}$ & $\begin{array}{l}\text { Crutcho Ck Tribat Reno Midwest City } \\
N \text { Canadian } R \text { nr Harrah } \\
N \text { Canadian R nr Harrah } \\
\text { N Canadian R nr Harrah } \\
\text { N Canadian R nr Harrah }\end{array}$ & $\begin{array}{l}\text { CSR } \\
\text { STR } \\
\text { QWP } \\
\text { QWMP } \\
\text { SED }\end{array}$ & $\begin{array}{l}\text { CNP } \\
\text { CNP } \\
S\end{array}$ & OBS & $\begin{array}{l}X G \\
X G \\
A\end{array}$ & $\begin{array}{l}1.41 \\
13501 \\
13501 \\
13501 \\
13501\end{array}$ & $\begin{array}{l}1976-81 \\
1968- \\
1969- \\
1973-79 \\
1983-\end{array}$ \\
\hline $\begin{array}{l}\sigma 724165 \emptyset \\
\varnothing 72416 \varnothing \emptyset \\
6724188 \emptyset \\
672426 \sigma \emptyset \\
\sigma 7242 \varnothing \varnothing \emptyset\end{array}$ & $\begin{array}{l}\text { N Canadian R nr Harrah } \\
\text { Lake Shawnee nr Shawnee } \\
\text { Sand Ck nr Cromwell } \\
N \text { Canadian R nr Wetumka } \\
\text { N Canadian R nr Wetumka }\end{array}$ & $\begin{array}{l}\text { QWD } \\
\text { RESU } \\
\text { CS } \\
\text { STR } \\
\text { QWP }\end{array}$ & BCNP & $D C P$ & $\begin{array}{l}X G \\
H G \\
C \\
J G A\end{array}$ & $\begin{array}{c}13501 . \\
34.0 \\
9.48 \\
14290 . \\
14290\end{array}$ & $\begin{array}{l}1968- \\
1957-78 \\
1964-86 \\
1937- \\
1952,54-\end{array}$ \\
\hline $\begin{array}{l}\sigma 7242 \sigma \sigma \sigma \\
\sigma 7242 \sigma \sigma \sigma \\
\sigma 7242 \sigma \sigma \sigma \\
\sigma 7242 \varnothing 5 \emptyset \\
\sigma 724208 \sigma\end{array}$ & $\begin{array}{l}N \text { Canadian } R \text { nr Wetumka } \\
N \text { Canadian } R \text { nr Wetumka } \\
N \text { Canadian } R \text { nr Wetumka } \\
\text { Wewoka Ck } n r \text { Wowoka } \\
\text { Little Wewoka Ck nr Wetumka }\end{array}$ & $\begin{array}{l}\text { QWMP } \\
\text { SED } \\
\text { QWD } \\
\text { QWP } \\
\text { QWP }\end{array}$ & $\begin{array}{l}\text { CNP } \\
S \\
P \\
C P \\
C P\end{array}$ & & $\begin{array}{l}A E \\
\text { JGAE }\end{array}$ & $\begin{array}{l}14290 \\
14290 \\
14290\end{array}$ & $\begin{array}{l}1973-79 \\
1977- \\
1953- \\
1962-63 \\
1962-63\end{array}$ \\
\hline $\begin{array}{l}\emptyset 7242 \varnothing 9 \emptyset \\
\varnothing 72421 \varnothing \emptyset \\
\emptyset 72421 \varnothing \emptyset \\
\emptyset 72421 \varnothing 9 \\
\varnothing 724216 \varnothing\end{array}$ & $\begin{array}{l}\text { Grief Ck nr Wetumka } \\
\text { Wewoka Ck nr Wetumka } \\
\text { Wewoka Ck nr Wotumka } \\
\text { Fish Ck nr Wetumka } \\
\text { Alabama Ck nr Weleetka }\end{array}$ & $\begin{array}{l}\text { QWP } \\
\text { STR } \\
\text { QWP } \\
\text { QWP } \\
\text { CS }\end{array}$ & $\begin{array}{l}C P \\
C P \\
C P\end{array}$ & & HGJ & $\begin{array}{l}396 . \\
396 . \\
16.5\end{array}$ & $\begin{array}{l}1962-63 \\
1960-63,66-67 \\
1952-57,60-64 \\
1960 \\
1965-\end{array}$ \\
\hline $\begin{array}{l}\emptyset 724216 \emptyset \\
\sigma 724218 \emptyset \\
\emptyset 724219 \emptyset \\
\sigma 72422 \varnothing \emptyset \\
67242217\end{array}$ & $\begin{array}{l}\text { A labama Ck nr Weleetka } \\
\text { Stidham Ck Trib nr Dustin } \\
N \text { Canadian } R \text { nr Pierce } \\
\text { Deep Fk at Portland, OKC } \\
\text { Deep Fk at Pennsylvania Ave, OKC }\end{array}$ & $\begin{array}{l}\text { CSR } \\
\text { CS } \\
\text { QWP } \\
\text { CSR } \\
\text { QWP }\end{array}$ & $\begin{array}{l}C P \\
C\end{array}$ & & & $\begin{array}{r}16.5 \\
2.56 \\
2.98\end{array}$ & $\begin{array}{l}1966-83 \\
1964-76 \\
196 \emptyset-63 \\
1974-81 \\
196 \emptyset-62\end{array}$ \\
\hline $\begin{array}{l}\emptyset 7242219 \\
\emptyset 724222 \emptyset \\
\varnothing 724227 \emptyset \\
\emptyset 72423 \emptyset \emptyset \\
\varnothing 724234 \emptyset\end{array}$ & $\begin{array}{l}\text { Deep Fk Trib at NW } 5 \varnothing \text {, OKC } \\
\text { Deep Fk at Eastern, OKC } \\
\text { Spring Ck at Pine Oak Dr, Edmond } \\
\text { Deep Fk at Witcher } \\
\text { Arcadia L nr Arcadia }\end{array}$ & $\begin{array}{l}\text { CSR } \\
\text { CSR } \\
\text { CSR } \\
\text { QWP } \\
\text { RES }\end{array}$ & CNOP & $D C P$ & C & $\begin{array}{c}2.65 \\
28.2 \\
1.32\end{array}$ & $\begin{array}{l}1976-81 \\
1976-81 \\
1976-81 \\
1980-62,73 \\
1986-\end{array}$ \\
\hline $\begin{array}{l}\emptyset 7242345 \\
67242345 \\
\emptyset 7242345 \\
\emptyset 724235 \emptyset \\
\emptyset 724235 \emptyset\end{array}$ & $\begin{array}{l}\text { Deep Fork blw Arcadia L nr Arcadia } \\
\text { Deep Fork blw Arcadia L nr Arcadia } \\
\text { Deep Fork blw Arcadia L nr Arcadia } \\
\text { Deep Fk } n r \text { Arcadia } \\
\text { Deep Fk } n r \text { Arcadia }\end{array}$ & $\begin{array}{l}\text { STR } \\
\text { QWP } \\
\text { SED } \\
\text { STR } \\
\text { QWP }\end{array}$ & $\begin{array}{l}\text { QWP } \\
\text { CNP } \\
\text { CNP }\end{array}$ & OBS & $\begin{array}{l}c \\
C \\
C \\
C \\
C\end{array}$ & $\begin{array}{l}165 . \\
165 .\end{array}$ & $\begin{array}{l}1987- \\
1987- \\
1987- \\
1969-87 \\
1976-87\end{array}$ \\
\hline
\end{tabular}

* Continuous streamflow records for this period.

** Some records in intervening years.

*** Some records may have been collected previously. 
Tablo 2.--Station number listing of current and historical gaging stations maintained by the U.S. Geological Survey

September 30, 1987--Continued

[Symbols defined on last page of report]

\begin{tabular}{|c|c|c|c|c|c|c|c|}
\hline $\begin{array}{l}\text { Station } \\
\text { Number }\end{array}$ & Station & $\begin{array}{l}\text { Type } \\
\text { of } \\
\text { Data }\end{array}$ & $\begin{array}{l}\text { Q.W. } \\
\text { Para- } \\
\text { meters }\end{array}$ & $\begin{array}{l}\text { Stage } \\
\text { Source }\end{array}$ & $\begin{array}{l}\text { Coop- } \\
\text { orator }\end{array}$ & $\begin{array}{c}\text { Drainage } \\
\text { Area } \\
\left(m i l e^{2}\right)\end{array}$ & Period of Record \\
\hline $\begin{array}{l}\emptyset 724235 \emptyset \\
\emptyset 724235 \emptyset \\
\emptyset 724237 \emptyset \\
\emptyset 724237 \emptyset \\
\emptyset 724238 \emptyset\end{array}$ & $\begin{array}{l}\text { Deep Fk nr Arcadia } \\
\text { Deep Fk nr Arcadia } \\
\text { Wildhorse Ck Trib nr Luther } \\
\text { Wildhorse Ck Trib nr Luther } \\
\text { Deep Fk at Warwick }\end{array}$ & $\begin{array}{l}\text { QWMP } \\
\text { SED } \\
\text { CSR } \\
\text { CS } \\
\text { STR }\end{array}$ & $\begin{array}{l}\text { CNP } \\
S\end{array}$ & DCP & $\begin{array}{l}\mathrm{C} \\
\mathrm{JG} \\
\mathrm{C}\end{array}$ & $\begin{array}{l}105 . \\
105 . \\
2.12 \\
2.12 \\
522\end{array}$ & $\begin{array}{l}1973-79 \\
1978-87 \\
1974-76 \\
1974- \\
1984-\end{array}$ \\
\hline $\begin{array}{l}\emptyset 724238 \emptyset \\
\emptyset 72424 \varnothing \emptyset \\
\varnothing 72425 \varnothing \emptyset \\
\emptyset 72425 \emptyset \emptyset \\
\emptyset 7243 \varnothing \emptyset \emptyset\end{array}$ & $\begin{array}{l}\text { Deep Fk at Warwick } \\
\text { Deep Fk nr Chandler } \\
\text { Bell cow Ck at Chandler } \\
\text { Bell cow Ck at Chandler } \\
\text { Dry Ck nr Kendrick }\end{array}$ & $\begin{array}{l}\text { SED } \\
\text { QWP } \\
\text { STR } \\
\text { QWP } \\
\text { STR }\end{array}$ & $\begin{array}{l}\mathrm{S} \\
\mathrm{CP} \\
\mathrm{CP}\end{array}$ & & $c$ & $\begin{array}{l}622 . \\
46.0 \\
46.0 \\
69.0\end{array}$ & $\begin{array}{l}1984- \\
1960-62 \\
1948-55 \\
1949-54 \\
1965-\end{array}$ \\
\hline $\begin{array}{l}\emptyset 7243 \emptyset \emptyset \emptyset \\
\emptyset 724345 \emptyset \\
\emptyset 72435 \emptyset \emptyset \\
\emptyset 72435 \varnothing \emptyset \\
\emptyset 72436 \varnothing \emptyset\end{array}$ & $\begin{array}{l}\text { Dry Ck nr Kendrick } \\
\text { Little Deep Fk Ck nr Edna } \\
\text { Deep Fk nr Beggs } \\
\text { Deop Fk nr Beggs } \\
\text { Deep Fk nr Beggs }\end{array}$ & $\begin{array}{l}\text { QWP } \\
\text { QWP } \\
\text { STR } \\
\text { QWP } \\
\text { QWMP }\end{array}$ & $\begin{array}{l}\text { COP } \\
\text { CP } \\
\text { BCNP } \\
\text { CNP }\end{array}$ & DCP & $\begin{array}{l}\text { C } \\
\text { JGAE }\end{array}$ & $\begin{array}{l}69.0 \\
2018 \\
2018 \\
2018\end{array}$ & $\begin{array}{l}* * 1955,70-73 \\
1951-67,60-62 \\
1938- \\
1952- \\
1973-79\end{array}$ \\
\hline $\begin{array}{l}\emptyset 72435 \varnothing \emptyset \\
\emptyset 72435 \varnothing \emptyset \\
\emptyset 724355 \emptyset \\
\emptyset 724365 \varnothing \\
\varnothing 7244 \varnothing \varnothing \emptyset\end{array}$ & $\begin{array}{l}\text { Deep Fk } n r \text { Beggs } \\
\text { Deep Fk } n r \text { Beggs } \\
\text { Adams Ck } n r \text { Beggs } \\
\text { Adams Ck } n r \text { Beggs } \\
\text { Deep Fk } n r \text { Dewar }\end{array}$ & $\begin{array}{l}\text { SED } \\
\text { QWD } \\
\text { CS } \\
\text { CSR } \\
\text { STRP }\end{array}$ & $\begin{array}{l}S \\
P\end{array}$ & & $\begin{array}{l}\text { CAE } \\
J G A \\
H G\end{array}$ & $\begin{array}{l}2018 . \\
2018 . \\
5.90 \\
5.90 \\
2307 .\end{array}$ & $\begin{array}{l}1978- \\
1951- \\
1965-85 \\
1965-76 \\
1938-50\end{array}$ \\
\hline $\begin{array}{l}\emptyset 7244 \varnothing \emptyset \emptyset \\
\varnothing 72442 \varnothing \emptyset \\
\emptyset 72445 \varnothing \emptyset \\
\varnothing 72445 \varnothing \emptyset \\
\emptyset 724465 \emptyset\end{array}$ & $\begin{array}{l}\text { Deep Fk nr Dewar } \\
\text { Deep Fk nr Pierce } \\
N \text { Canadian R nr Eufaula } \\
N \text { Canadian } R \text { nr Eufaula } \\
\text { Longtown Ck nr Enterprise }\end{array}$ & $\begin{array}{l}\text { QWD } \\
\text { QWP } \\
\text { STR } \\
\text { QWP } \\
\text { QWP }\end{array}$ & $\begin{array}{l}\mathrm{CP} \\
\mathrm{CP} \\
\mathrm{CP} \\
\mathrm{CP}\end{array}$ & & & $\begin{array}{l}2307 \\
2648 \\
17657 \\
17657\end{array}$ & $\begin{array}{l}1949-51 \\
1960-63 \\
1960-62 \\
1952-63,60,61 \\
1952-63\end{array}$ \\
\hline $\begin{array}{l}\emptyset 724479 \emptyset \\
\varnothing 72448 \varnothing \emptyset \\
\emptyset 72448 \varnothing \emptyset \\
\emptyset 724490 \emptyset \\
\varnothing 7245 \varnothing \emptyset \emptyset\end{array}$ & $\begin{array}{l}\text { Brooken Ck nr Enterprise } \\
\text { Eufaula Lake } n r \text { Brooken } \\
\text { Eufaula Lake } n r \text { Brooken } \\
\text { Canadian } R \text { at L Eufaula TW } \\
\text { Canadian } R n r \text { Whitefield }\end{array}$ & $\begin{array}{l}\text { CS } \\
\text { RES } \\
\text { QWD } \\
\text { RESU } \\
\text { STR }\end{array}$ & $\mathrm{CP}$ & $\begin{array}{l}D C P \\
D C P\end{array}$ & $\begin{array}{l}c \\
c \\
c\end{array}$ & $\begin{array}{l}\quad 5.66 \\
47522 \\
47522 \\
47576\end{array}$ & $\begin{array}{l}1964-71 \\
1964- \\
1965-67 \\
1986- \\
1938-\end{array}$ \\
\hline 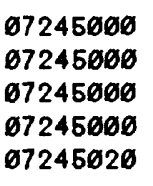 & $\begin{array}{l}\text { Canadian } R n r \text { Whitefield } \\
\text { Canadian } R n r \text { Whitefield } \\
\text { Canadian } R n r \text { Whitefield } \\
\text { Canadian } R n r \text { Whitefield } \\
\text { Taloka Ck at Stigler }\end{array}$ & $\begin{array}{l}\text { QWP } \\
\text { QWMP } \\
\text { SED } \\
\text { QWD } \\
\text { PR }\end{array}$ & $\begin{array}{l}\text { BCNP } \\
\text { CNP } \\
S \\
P\end{array}$ & OBS & $\begin{array}{l}J G A \\
\hat{J}_{G A}\end{array}$ & $\begin{array}{l}47576 \\
47576 \\
47576 \\
47576 \\
\quad 3.98\end{array}$ & $\begin{array}{l}1947-64,67-86 \\
1973-77 \\
1975- \\
1944-45,46-64,66-86 \\
1978-81\end{array}$ \\
\hline $\begin{array}{l}\emptyset 7245 \varnothing 2 \emptyset \\
\varnothing 7245 \varnothing 2 \emptyset \\
\emptyset 7245625 \\
\emptyset 7245025 \\
\emptyset 7245 \emptyset 25\end{array}$ & $\begin{array}{l}\text { Taloka Ck at Stigler } \\
\text { Taloka Ck at Stigler } \\
\text { Taloka Ck Trib nr Stigler } \\
\text { Taloka Ck Trib nr Stigler } \\
\text { Taloka Ck Trib nr Stigler }\end{array}$ & $\begin{array}{l}\text { QWP } \\
\text { SED } \\
\text { PR } \\
\text { QWP } \\
\text { SED }\end{array}$ & $\begin{array}{l}C N P \\
S \\
C N \\
P \\
S\end{array}$ & & & $\begin{array}{l}3.98 \\
3.98 \\
2.04 \\
2.04 \\
2.04\end{array}$ & $\begin{array}{l}1978-81 \\
1978-81 \\
1979-81 \\
1978-81 \\
1979-81\end{array}$ \\
\hline
\end{tabular}

* Continuous streamflow records for this period.

* Some records in intervening years.

*** Some records may have been collected previously. 
Table 2.--Station number listing of current and historical gaging stations maintained by the U.S. Geological Survey

September s0, 1987--Continued

[Symbols defined on last page of report]

\begin{tabular}{|c|c|c|c|c|c|c|c|}
\hline $\begin{array}{r}\text { Station } \\
\text { Number }\end{array}$ & Station & $\begin{array}{l}\text { Type } \\
\text { of } \\
\text { Data }\end{array}$ & $\begin{array}{l}\text { Q.W. } \\
\text { Para- } \\
\text { meters }\end{array}$ & $\begin{array}{l}\text { Stage } \\
\text { Source }\end{array}$ & $\begin{array}{l}\text { Coop- } \\
\text { erator }\end{array}$ & $\begin{array}{c}\text { Drainage } \\
\text { Area } \\
\left(m i l e^{2}\right)\end{array}$ & Period of Record \\
\hline $\begin{array}{l}\varnothing 7245 \varnothing 3 \emptyset \\
\varnothing 7245 \varnothing 3 \emptyset \\
\varnothing 724563 \emptyset \\
\varnothing 7246 \varnothing 4 \emptyset \\
\varnothing 724564 \emptyset\end{array}$ & $\begin{array}{l}\text { Taloka Ck nr Stigler } \\
\text { Taloka Ck nr Stigler } \\
\text { Taloka Ck nr Stigler } \\
\text { Jackson Ck nr Stigler } \\
\text { Jackson Ck nr Stigler }\end{array}$ & $\begin{array}{l}\text { STR } \\
\text { QWP } \\
\text { SED } \\
\text { PR } \\
\text { QWP }\end{array}$ & $\begin{array}{l}\text { CNP } \\
S \\
\text { CNP }\end{array}$ & & & $\begin{array}{l}20.1 \\
20.1 \\
20.1 \\
7.33 \\
7.33\end{array}$ & $\begin{array}{l}1978-81 \\
1978-81 \\
1978-81 \\
1980-81 \\
198 \emptyset-81\end{array}$ \\
\hline $\begin{array}{l}\varnothing 724564 \emptyset \\
\varnothing 724569 \emptyset \\
\varnothing 7245 \varnothing 9 \emptyset \\
\varnothing 7245119 \\
\varnothing 724556 \emptyset\end{array}$ & $\begin{array}{l}\text { Jackson Ck nr Stigler } \\
\text { Vian Ck nr Vian } \\
\text { Vian Ck nr Vian } \\
\text { Little Vian Ck nr Vian } \\
\text { Sallisaw Ck nr Sallisaw }\end{array}$ & $\begin{array}{l}\text { SED } \\
\text { CS } \\
\text { QWP } \\
\text { QWP } \\
\text { STRP }\end{array}$ & $\begin{array}{l}S \\
C P \\
C P\end{array}$ & & & $\begin{array}{l}7.33 \\
19.6 \\
19.6 \\
182\end{array}$ & $\begin{array}{l}198 \emptyset-81 \\
1966-72 \\
1968-69 \\
196 \varnothing \\
1942-76\end{array}$ \\
\hline $\begin{array}{l}\varnothing 72456 \varnothing \emptyset \\
\varnothing 72455 \varnothing \emptyset \\
\varnothing 724558 \emptyset \\
\varnothing 724568 \emptyset \\
\varnothing 724569 \emptyset\end{array}$ & $\begin{array}{l}\text { Sallisaw Ck nr Sallisaw } \\
\text { Sallisaw Ck nr Sallisaw } \\
\text { Mule Ck at SR } 31 \mathrm{nr} \text { McCurtain } \\
\text { Mule Ck at SR } 31 \mathrm{nr} \text { McCurtain } \\
\text { Mule Ck nr McCurtain }\end{array}$ & $\begin{array}{l}\text { QWP } \\
\text { QWMP } \\
\text { QWP } \\
\text { PR } \\
\text { QWP }\end{array}$ & $\begin{array}{l}\text { CP } \\
C N P \\
C P \\
C P\end{array}$ & & & $\begin{array}{l}182 . \\
182 .\end{array}$ & $\begin{array}{l}1952-63 \\
1977 \\
1981-82 \\
1981-82 \\
1979-82\end{array}$ \\
\hline $\begin{array}{l}\emptyset 724569 \emptyset \\
\varnothing 7246591 \\
\emptyset 7246591 \\
\emptyset 7245692 \\
\varnothing 7245692\end{array}$ & $\begin{array}{l}\text { Mule Ck nr McCurtain } \\
\text { E Pond Outlet to Mule Ck nr McCurtain } \\
\text { E Pond Outlet to Mule Ck nr McCurtain } \\
\text { Mule Ck nr MeCurtain } \\
\text { Mule Ck nr McCurtain }\end{array}$ & $\begin{array}{l}\text { PR } \\
\text { QWP } \\
\text { PR } \\
\text { QWP } \\
\text { PR }\end{array}$ & $\begin{array}{l}C P \\
C P\end{array}$ & & & & $\begin{array}{l}1979-82 \\
1979-82 \\
1979-82 \\
1981-82 \\
1981-82\end{array}$ \\
\hline $\begin{array}{l}\emptyset 7245594 \\
\emptyset 7246594 \\
\emptyset 72467 \emptyset 3 \\
\emptyset 724606 \emptyset \\
\varnothing 7246 \varnothing 6 \varnothing\end{array}$ & $\begin{array}{l}\text { Mule Ck nr McCurtain } \\
\text { Mule Ck nr McCurtain } \\
\text { Sans Bois Ck nr Kinta } \\
\text { Sans Bois Ck nr Keota } \\
\text { Sans Bois Ck nr Keota }\end{array}$ & $\begin{array}{l}\text { QWP } \\
\text { PR } \\
\text { QWP } \\
\text { STR } \\
\text { QWP }\end{array}$ & $\begin{array}{l}\mathrm{CP} \\
\mathrm{CP} \\
\mathrm{CP}\end{array}$ & & & $\begin{array}{l}346 \\
346\end{array}$ & $\begin{array}{l}1981-82 \\
1981-82 \\
1961 \\
1938-42 \\
1958-63\end{array}$ \\
\hline $\begin{array}{l}\emptyset 724631 \emptyset \\
\emptyset 72464 \varnothing \emptyset \\
\varnothing 72464 \varnothing \emptyset \\
\emptyset 72464 \varnothing \emptyset \\
\varnothing 72465 \varnothing \emptyset\end{array}$ & $\begin{array}{l}R \text { S Kerr L D nr Sallisaw (Ark US) } \\
R \text { S Kerr L D nr Sallisaw (Ark R) } \\
R \text { S Kerr L D nr Sallisaw (Ark R) } \\
R \text { S Kerr L D nr Sallisaw (Ark R) } \\
\text { Arkansas R nr Sallisaw }\end{array}$ & $\begin{array}{l}\text { RESU } \\
\text { QWP } \\
\text { QWMP } \\
\text { RESU } \\
\text { STRP }\end{array}$ & $\begin{array}{l}C P \\
C N P\end{array}$ & DCP & $\begin{array}{l}\mathrm{C} \\
\mathrm{JG} \\
\mathrm{C}\end{array}$ & $\begin{array}{l}147756 \\
147756 \\
147756 \\
147757\end{array}$ & $\begin{array}{l}1986- \\
1970- \\
1976-79 \\
1986- \\
1948-76\end{array}$ \\
\hline $\begin{array}{l}\emptyset 72465 \emptyset \emptyset \\
\varnothing 72466 \emptyset \emptyset \\
\varnothing 72466 \emptyset \emptyset \\
\varnothing 724661 \emptyset \\
\varnothing 7246615\end{array}$ & $\begin{array}{l}\text { Arkansas } R \text { nr Sallisaw } \\
\text { Cache Ck nr Cowlington } \\
\text { Cache Ck nr Cowlington } \\
\text { Pecan Ck nr Spiro } \\
\text { Coal Ck nr Spiro }\end{array}$ & $\begin{array}{l}\text { QWD } \\
\text { CS } \\
\text { QWP } \\
\text { CSR } \\
\text { STR }\end{array}$ & $\begin{array}{l}C P \\
C P\end{array}$ & & & $\begin{array}{r}147757 . \\
20.6 \\
20.6 \\
0.96 \\
18.1\end{array}$ & $\begin{array}{l}195769-63 \\
1964-72 \\
1959-61 \\
1964-76 \\
1978-82\end{array}$ \\
\hline $\begin{array}{l}\emptyset 7246615 \\
67246615 \\
\varnothing 7246615 \\
6724663 \emptyset \\
6724676 \varnothing\end{array}$ & $\begin{array}{l}\text { Coal Ck nr Spiro } \\
\text { Coal Ck nr Spiro } \\
\text { Coal Ck nr Spiro } \\
\text { Big Black Fox Ck nr Long } \\
\text { W D Mayo L } D r \text { Braden US }\end{array}$ & $\begin{array}{l}\text { QWH } \\
\text { SED } \\
\text { QWP } \\
\text { CS } \\
\text { RESU }\end{array}$ & $\begin{array}{l}P \\
S \\
\text { BCNP }\end{array}$ & & $\begin{array}{l}H G \\
C\end{array}$ & $\begin{array}{c}18.1 \\
18.1 \\
18.1 \\
6.32\end{array}$ & $\begin{array}{l}1978-82 \\
1978-81 \\
1978-81 \\
1964-85 \\
1986-\end{array}$ \\
\hline
\end{tabular}

* Continuous streamflow records for this period.

** Some records in intervening years.

*** Some records may have been collected previously. 
Table 2.--Station number listing of current and historical gaging stations maintained by the U.S. Geological Survey

September $90,1987-$-Continued

[Symbols defined on last page of report]

\begin{tabular}{|c|c|c|c|c|c|c|c|}
\hline $\begin{array}{c}\text { Station } \\
\text { Number }\end{array}$ & Station & $\begin{array}{c}\text { Type } \\
\text { of } \\
\text { Data }\end{array}$ & $\begin{array}{l}\text { Q.W. } \\
\text { Para- } \\
\text { meters }\end{array}$ & $\begin{array}{l}\text { Stage } \\
\text { Source }\end{array}$ & $\begin{array}{l}\text { Coop- } \\
\text { erator }\end{array}$ & $\begin{array}{c}\text { Drainage } \\
\text { Area } \\
\left(m i l e^{2}\right)\end{array}$ & Period of Record \\
\hline $\begin{array}{l}\emptyset 724671 \emptyset \\
\emptyset 7247 \emptyset \emptyset \emptyset \\
\emptyset 724735 \emptyset \\
\emptyset 724745 \emptyset \\
\emptyset 724745 \emptyset\end{array}$ & $\begin{array}{l}\text { W D Mayo L D nr Braden DS } \\
\text { Poteau } R \text { at Cauthron AR } \\
\text { Poteau } R \text { nr Heavener } \\
\text { Fourche Maline nr Wilburton } \\
\text { Fourche Maline nr Wilburton }\end{array}$ & $\begin{array}{l}\text { RESU } \\
\text { QWMP } \\
\text { QWMP } \\
\text { STR } \\
\text { QWH }\end{array}$ & $\begin{array}{l}\text { CNP } \\
\text { CNP } \\
P\end{array}$ & DCP & $\mathrm{C}$ & $\begin{array}{l}263 . \\
56.2 \\
56.2\end{array}$ & $\begin{array}{l}1986- \\
1973-75 \\
1978-79 \\
1978-81 \\
1978-80\end{array}$ \\
\hline $\begin{array}{l}\emptyset 724745 \emptyset \\
\emptyset 724745 \emptyset \\
\emptyset 72475 \emptyset \emptyset \\
\emptyset 72475 \emptyset \emptyset \\
\emptyset 72475 \varnothing \emptyset\end{array}$ & $\begin{array}{l}\text { Fourche Maline nr Wilburton } \\
\text { Fourche Maline nr Wilburton } \\
\text { Fourche Maline nr Red Oak } \\
\text { Fourche Maline nr Red Dak } \\
\text { Fourche Maline nr Red Oak }\end{array}$ & $\begin{array}{l}\text { SED } \\
\text { QWP } \\
\text { STR } \\
\text { QWP } \\
\text { QWMP }\end{array}$ & $\begin{array}{l}S \\
\text { CNP } \\
\text { CP } \\
\text { CNP }\end{array}$ & & $J G$ & $\begin{array}{l}56.2 \\
56.2 \\
122 . \\
122 . \\
122 .\end{array}$ & $\begin{array}{l}1978-81 \\
1978-81 \\
1938- \\
1952,54,56-60,63 \\
1978-79\end{array}$ \\
\hline $\begin{array}{l}\emptyset 724756 \emptyset \\
\varnothing 724756 \varnothing \\
\varnothing 724756 \varnothing \\
\varnothing 724756 \varnothing \\
\varnothing 7248 \emptyset \emptyset \emptyset\end{array}$ & $\begin{array}{l}\text { Red Dak Ck nr Red Dak } \\
\text { Red Dak Ck nr Red Dak } \\
\text { Red Dak Ck nr Red Dak } \\
\text { Red Oak Ck nr Red Oak } \\
\text { Wister Lake nr Wister }\end{array}$ & $\begin{array}{l}\text { STR } \\
\text { QWP } \\
\text { SED } \\
\text { QWH } \\
\text { RES }\end{array}$ & $\begin{array}{l}\text { CNP } \\
S \\
P\end{array}$ & $D C P$ & C & $\begin{array}{l}13.1 \\
13.1 \\
13.1 \\
13.1 \\
993\end{array}$ & $\begin{array}{l}1978-82 \\
1978-81 \\
1978-81 \\
1980-81 \\
1949-\end{array}$ \\
\hline $\begin{array}{l}\emptyset 7248 \emptyset \emptyset \emptyset \\
\emptyset 72485 \varnothing \emptyset \\
\emptyset 72485 \emptyset \emptyset \\
\emptyset 72485 \emptyset \emptyset \\
\emptyset 72486 \varnothing \emptyset\end{array}$ & $\begin{array}{l}\text { Wister Lake nr Wister } \\
\text { Poteau } R n r \text { Wister } \\
\text { Poteau R nr Wister } \\
\text { Poteau R nr Wister } \\
\text { Caston Ck at Wister }\end{array}$ & $\begin{array}{l}\text { QWP } \\
\text { STGU } \\
\text { QWP } \\
\text { QWMP } \\
\text { STR }\end{array}$ & $\begin{array}{l}\mathrm{CP} \\
\mathrm{CP} \\
\mathrm{CNP}\end{array}$ & & C & $\begin{array}{l}993 . \\
993 . \\
993 . \\
993 . \\
72.9\end{array}$ & $\begin{array}{l}1960-64 \\
1938- \\
1948,52,55-59 \\
1973-79 \\
1978-82\end{array}$ \\
\hline $\begin{array}{l}\emptyset 72486 \emptyset \emptyset \\
\varnothing 72486 \varnothing \emptyset \\
\emptyset 724862 \emptyset \\
\varnothing 724862 \emptyset \\
\varnothing 724862 \emptyset\end{array}$ & $\begin{array}{l}\text { Caston } \mathrm{Ck} \text { at Wister } \\
\text { Caston } \mathrm{Ck} \text { at Wister } \\
\text { Morris Ck at Howe } \\
\text { Morris Ck at Howe } \\
\text { Morris Ck at Howe }\end{array}$ & $\begin{array}{l}\text { QWP } \\
\text { SED } \\
\text { STR } \\
\text { QWP } \\
\text { SED }\end{array}$ & $\begin{array}{l}\text { BCNP } \\
S \\
\text { CNP } \\
S\end{array}$ & & & $\begin{array}{l}72.9 \\
72.9 \\
19.4 \\
19.4 \\
19.4\end{array}$ & $\begin{array}{l}1978-81 \\
1978-81 \\
1978-81 \\
1978-81 \\
1978-81\end{array}$ \\
\hline $\begin{array}{l}\emptyset 72487 \varnothing \emptyset \\
\varnothing 72487 \varnothing \emptyset \\
\varnothing 72487 \varnothing \emptyset \\
\varnothing 72490 \varnothing \emptyset \\
\varnothing 72490 \varnothing \emptyset\end{array}$ & $\begin{array}{l}\text { Sugarloaf } C k n r \text { Monroe } \\
\text { Sugarloaf } C k n r \text { Monroe } \\
\text { Sugarloaf } C k n r \text { Monroe } \\
\text { Poteau } R \text { at Poteau } \\
\text { Poteau R at Poteau }\end{array}$ & $\begin{array}{l}\text { STR } \\
\text { QWP } \\
\text { SED } \\
\text { STR } \\
\text { QWP }\end{array}$ & $\begin{array}{l}\text { CNP } \\
\mathrm{S} \\
\mathrm{CP}\end{array}$ & & & $\begin{array}{l}53.6 \\
53.6 \\
53.6 \\
1240 . \\
1246 .\end{array}$ & $\begin{array}{l}1978-81 \\
1978-81 \\
1978-81 \\
1938-45 \\
1944-45\end{array}$ \\
\hline 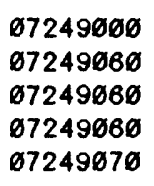 & $\begin{array}{l}\text { Poteau R at Poteau } \\
\text { Brazil Ck nr Red Dak } \\
\text { Brazil Ck nr Red Dak } \\
\text { Brazil Ck nr Red Dak } \\
\text { Rock Ck nr Red Oak }\end{array}$ & $\begin{array}{l}\text { STGU } \\
\text { PR } \\
\text { QWP } \\
\text { SED } \\
\text { PR }\end{array}$ & $\begin{array}{l}\text { CNP } \\
\mathrm{S}\end{array}$ & & C & $\begin{array}{r}1246 . \\
2.74 \\
2.74 \\
2.74 \\
12.0\end{array}$ & $\begin{array}{l}1986- \\
1978-81 \\
1978-81 \\
1978-81 \\
1978-81\end{array}$ \\
\hline $\begin{array}{l}\emptyset 724907 \emptyset \\
\emptyset 724907 \emptyset \\
\varnothing 7249073 \\
\emptyset 7249073 \\
\emptyset 7249073\end{array}$ & $\begin{array}{l}\text { Rock Ck nr Red Oak } \\
\text { Rock Ck nr Red Oak } \\
\text { Brazil Ck nr Lodi } \\
\text { Brazil Ck nr Lodi } \\
\text { Brazil Ck nr Lodi }\end{array}$ & $\begin{array}{l}\text { QWP } \\
\text { SED } \\
\text { PR } \\
\text { QWP } \\
\text { SED }\end{array}$ & $\begin{array}{l}\text { CNP } \\
S \\
\text { CNP } \\
S\end{array}$ & & & $\begin{array}{l}12.0 \\
12.0 \\
28.8 \\
28.8 \\
28.8\end{array}$ & $\begin{array}{l}1978-81 \\
1978-81 \\
1980-81 \\
1980-81 \\
1980-81\end{array}$ \\
\hline
\end{tabular}

* Continuous streamflow records for this period.

* Some records in intervening years.

*** Some records may have been collected previously. 
Table 2.--Station number listing of current and historical gaging stations maintained by the U.S. Geological Survey

September 30, 1987--Continued

[Symbols defined on last page of report]

\begin{tabular}{|c|c|c|c|c|c|c|c|}
\hline $\begin{array}{r}\text { Station } \\
\text { Number }\end{array}$ & Station Name & $\begin{array}{l}\text { Type } \\
\text { of } \\
\text { Data }\end{array}$ & $\begin{array}{l}\text { Q.W. } \\
\text { Para- } \\
\text { meters }\end{array}$ & $\begin{array}{l}\text { Stage } \\
\text { Source }\end{array}$ & $\begin{array}{l}\text { Coop- } \\
\text { erator }\end{array}$ & $\begin{array}{c}\text { Drainage } \\
\text { Area } \\
(m i l e)\end{array}$ & Period of Record \\
\hline $\begin{array}{l}\emptyset 7249 \varnothing 8 \emptyset \\
\emptyset 7249 \varnothing 8 \emptyset \\
\emptyset 7249 \varnothing 8 \emptyset \\
\emptyset 72491 \varnothing \emptyset \\
\emptyset 72491 \emptyset \emptyset\end{array}$ & $\begin{array}{l}\text { Brazil Ck nr Walls } \\
\text { Brazil Ck nr Walls } \\
\text { Brazil Ck nr Walls } \\
\text { Owl Ck nr McCurtain } \\
\text { Owl Ck nr McCurtain }\end{array}$ & $\begin{array}{l}\text { STR } \\
\text { QWP } \\
\text { SED } \\
\text { STR } \\
\text { QWP }\end{array}$ & $\begin{array}{l}\text { CNP } \\
S \\
C N P\end{array}$ & OBS & $A$ & $\begin{array}{l}69.1 \\
69.1 \\
69.1 \\
27.9 \\
27.9\end{array}$ & $\begin{array}{l}1978-81,84- \\
1978-81 \\
1978-81,84- \\
1978-81 \\
1978-81\end{array}$ \\
\hline $\begin{array}{l}\emptyset 72491 \emptyset \emptyset \\
\emptyset 72492 \varnothing \emptyset \\
\emptyset 72494 \varnothing \emptyset \\
\emptyset 72494 \varnothing \emptyset \\
\emptyset 72494 \emptyset \emptyset\end{array}$ & $\begin{array}{l}\text { OwI Ck nr McCurtain } \\
\text { Brazil Ck nr Panama } \\
\text { James Fk } n r \text { Hackett } A R \\
\text { James Fk } n r \text { Hackett } A R \\
\text { James Fk } n r \text { Hackett } A R\end{array}$ & $\begin{array}{l}\text { SED } \\
\text { QWP } \\
\text { STR } \\
\text { QWP } \\
\text { SED }\end{array}$ & $\begin{array}{l}S \\
\mathrm{CP} \\
\mathrm{CNP} \\
\mathrm{S}\end{array}$ & & $w$ & $\begin{array}{l}27.9 \\
148 . \\
148 . \\
148 .\end{array}$ & $\begin{array}{l}1978-81 \\
1960-61 \\
1958- \\
1960-61,76-81 \\
1976-81\end{array}$ \\
\hline $\begin{array}{l}\emptyset 724941 \emptyset \\
\emptyset 724941 \emptyset \\
\emptyset 724941 \emptyset \\
\emptyset 7249415 \\
\emptyset 7249415\end{array}$ & $\begin{array}{l}\text { James Fk nr Williams } \\
\text { James Fk nr Williams } \\
\text { James Fk nr Williams } \\
\text { Coal Ck Trib nr Bokoshe } \\
\text { Coal Ck Trib nr Bokoshe }\end{array}$ & $\begin{array}{l}\text { PR } \\
\text { QWP } \\
\text { SED } \\
\text { PR } \\
\text { QWP }\end{array}$ & $\begin{array}{l}\text { CNP } \\
S \\
\text { CNP }\end{array}$ & & & $\begin{array}{l}198 . \\
198 . \\
198 . \\
1.26 \\
1.26\end{array}$ & $\begin{array}{l}1976-81 \\
1976-81 \\
1976-81 \\
1976-79 \\
1976-79\end{array}$ \\
\hline $\begin{array}{l}\emptyset 7249415 \\
\emptyset 7249419 \\
\emptyset 7249419 \\
\emptyset 7249419 \\
\emptyset 7249419\end{array}$ & $\begin{array}{l}\text { Coal Ck Trib nr Bokoshe } \\
\text { Poteau } R \text { nr Panama } \\
\text { Poteau R nr Panama } \\
\text { Poteau R nr Panama } \\
\text { Poteau R nr Panama }\end{array}$ & $\begin{array}{l}\text { SED } \\
\text { CS } \\
\text { QWP } \\
\text { SED } \\
\text { STGU }\end{array}$ & $\begin{array}{l}S \\
\text { CNP } \\
S\end{array}$ & $D C P$ & $C$ & 1.26 & $\begin{array}{l}1976-79 \\
1976-79 \\
1976-79 \\
1977-79 \\
1986-\end{array}$ \\
\hline $\begin{array}{l}\emptyset 7249422 \\
\emptyset 7249422 \\
\emptyset 7249422 \\
\emptyset 7249438 \\
\emptyset 724944 \emptyset\end{array}$ & $\begin{array}{l}\text { Holi-Tuska Ck nr Panama } \\
\text { Holi-Tuska Ck nr Panama } \\
\text { Holi-Tuska Ck nr Panama } \\
\text { Poteau R nr Braden } \\
\text { Poteau R nr Fort Smith AR }\end{array}$ & $\begin{array}{l}\text { STRP } \\
\text { QWP } \\
\text { SED } \\
\text { QWP } \\
\text { QWMP }\end{array}$ & $\begin{array}{l}S \\
C P \\
C N P\end{array}$ & & & $\begin{array}{l}4.39 \\
4.39 \\
4.39\end{array}$ & $\begin{array}{l}1978-81 \\
1978-81 \\
1978-81 \\
1958-59,62-63 \\
1975-77\end{array}$ \\
\hline $\begin{array}{l}\emptyset 72498 \emptyset \emptyset \\
\emptyset 72498 \varnothing \emptyset \\
\emptyset 72499 \emptyset \emptyset \\
\emptyset 725 \varnothing 5 \emptyset \emptyset \\
\emptyset 725 \varnothing 5 \varnothing \emptyset\end{array}$ & 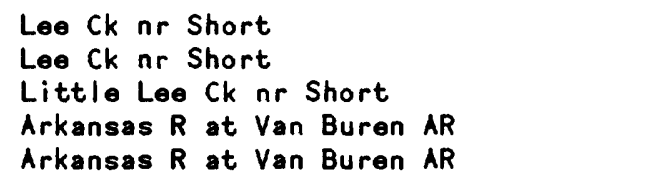 & $\begin{array}{l}\text { QWP } \\
\text { QWMP } \\
\text { QWMP } \\
\text { QWP } \\
\text { QWMP }\end{array}$ & $\begin{array}{l}\text { CP } \\
\text { CNP } \\
\text { CNP } \\
\text { CP } \\
\text { CNP }\end{array}$ & & & $\begin{array}{r}236 \\
236 \\
163 \\
150482 \\
150482\end{array}$ & $\begin{array}{l}1958-61 \\
1976-77 \\
1978-79 \\
1960-61 \\
1973-75\end{array}$ \\
\hline $\begin{array}{l}\emptyset 725050 \emptyset \\
\emptyset 725055 \emptyset \\
\emptyset 729850 \emptyset \\
\emptyset 7299565 \\
\emptyset 729957 \emptyset\end{array}$ & $\begin{array}{l}\text { Arkansas } R \text { at Van Buren } A R \\
\text { Arkansas } R \text { at Dam } 13 \text { nr Van Buren } \\
\text { Prairie Dog Town Fk Red } R \text { nr Brice TX } \\
\text { Red } R \text { nr Hollis } \\
\text { Red } R \text { nr Quanah TX }\end{array}$ & $\begin{array}{l}\text { STGU } \\
\text { QWMP } \\
\text { QWP } \\
\text { QWMP } \\
\text { QWMP }\end{array}$ & $\begin{array}{l}\text { CNP } \\
C \\
\text { CNP } \\
\text { CNP }\end{array}$ & DCP & C & $\begin{array}{r}150482 \\
150547 \\
6082 \\
8164 \\
8321\end{array}$ & $\begin{array}{l}1986- \\
1976-77 \\
196 \emptyset \\
1977 \\
1973-77\end{array}$ \\
\hline $\begin{array}{l}\emptyset 7299702 \\
\emptyset 72997 \emptyset 5 \\
\emptyset 7299710 \\
\emptyset 7299710 \\
\emptyset 7299710\end{array}$ & $\begin{array}{l}\text { Bitter Ck nr Hollis } \\
\text { Bitter Ck nr Hollis } \\
\text { Sandy Ck } n r \text { Eldorado } \\
\text { Sandy Ck nr Eldorado } \\
\text { Sandy Ck } n r \text { Eldorado }\end{array}$ & $\begin{array}{l}\text { STR } \\
\text { CS } \\
\text { STR } \\
\text { QWP } \\
\text { MISC }\end{array}$ & $C P$ & & $J G$ & $\begin{array}{l}11.3 \\
280 . \\
280 . \\
280 .\end{array}$ & $\begin{array}{l}1987- \\
1964-72 \\
1960-64 \\
1952-65,58,61-63 \\
1987-\end{array}$ \\
\hline
\end{tabular}

* Continuous streamflow records for this period.

* Some records in intervening years.

*** Some records may have been collected previously. 
Table 2.--Station number listing of current and historical gaging stations maintained by the U.S. Geological Survey

September 90, 1987--Continued

[Symbols defined on last page of report]

\begin{tabular}{|c|c|c|c|c|c|c|c|}
\hline $\begin{array}{r}\text { Station } \\
\text { Number }\end{array}$ & Station & $\begin{array}{c}\text { Type } \\
\text { of } \\
\text { Data }\end{array}$ & $\begin{array}{l}\text { Q.W. } \\
\text { Para- } \\
\text { meters }\end{array}$ & $\begin{array}{l}\text { Stage } \\
\text { Source }\end{array}$ & $\begin{array}{l}\text { Coop- } \\
\text { orator }\end{array}$ & $\begin{array}{c}\text { Drainage } \\
\text { Area } \\
(\text { mile })\end{array}$ & Period of Record \\
\hline $\begin{array}{l}\emptyset 729972 \emptyset \\
\emptyset 7299725 \\
\emptyset 729977 \emptyset \\
\emptyset 729978 \emptyset \\
\emptyset 729978 \emptyset\end{array}$ & $\begin{array}{l}\text { Mule Ck } n r \text { Eldorado } \\
\text { Eldorado Spring } n r \text { Eldorado } \\
\text { Gypsum Ck } n r \text { Creta } \\
\text { Gypsum Ck } n r \text { Olustee } \\
\text { Gypsum Ck } n r \text { Olustee }\end{array}$ & $\begin{array}{l}\text { CS } \\
\text { QWP } \\
\text { QWP } \\
\text { LF } \\
\text { QWP }\end{array}$ & $\begin{array}{l}C P \\
C P \\
C P\end{array}$ & & & $\begin{array}{l}3.84 \\
167 . \\
167 .\end{array}$ & $\begin{array}{l}1965-72 \\
1950 \\
1961 \\
1954-57,65-73 \\
1955-57\end{array}$ \\
\hline $\begin{array}{l}\emptyset 729978 \emptyset \\
6729978 \emptyset \\
\varnothing 730000 \emptyset \\
\varnothing 73 \varnothing \emptyset 15 \emptyset \\
\varnothing 73 \varnothing \emptyset 15 \emptyset\end{array}$ & $\begin{array}{l}\text { Gypsum Ck nr Olustee } \\
\text { Gypsum Ck } n r \text { Olustee } \\
\text { Salt Fk Red R nr Wellington TX } \\
\text { Bear Ck } n r \text { Vinson } \\
\text { Bear Ck nr Vinson }\end{array}$ & $\begin{array}{l}\text { MISC } \\
\text { STR } \\
\text { QWMP } \\
\text { CS } \\
\text { CSR }\end{array}$ & CNP & & $H G$ & $\begin{array}{r}107 \\
107 \\
1222 \\
7.24 \\
7.24\end{array}$ & $\begin{array}{l}1987- \\
1987- \\
1976-77 \\
1964-85 \\
1964-76\end{array}$ \\
\hline 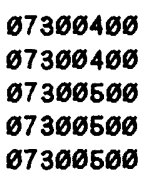 & $\begin{array}{l}\text { Salt Fk Red } R \text { nr Vinson } \\
\text { Salt Fk Red } R \text { nr Vinson } \\
\text { Salt Fk Red } R \text { at Mangum } \\
\text { Salt Fk Red } R \text { at Mangum } \\
\text { Salt Fk Red } R \text { at Mangum }\end{array}$ & $\begin{array}{l}\text { QWP } \\
\text { QWMP } \\
\text { STR } \\
\text { QWP } \\
\text { QWMP }\end{array}$ & $\begin{array}{l}\text { CP } \\
\text { CNP } \\
\text { CP } \\
\text { CNP }\end{array}$ & OBS & $J G$ & $\begin{array}{l}1421 . \\
1421 . \\
1566 . \\
1566 \\
1566\end{array}$ & $\begin{array}{l}1952-54,6 \varnothing-63 \\
1977 \\
1938- \\
* 1947-62,54,60-63 \\
1975-79\end{array}$ \\
\hline $\begin{array}{l}\emptyset 7300500 \\
\emptyset 7301000 \\
67301100 \\
\varnothing 7301100 \\
\emptyset 7301100\end{array}$ & $\begin{array}{l}\text { Salt Fk Red } R \text { at Mangum } \\
\text { Turkey Ck } n r \text { Olustee } \\
\text { Turkey Ck at Olustee } \\
\text { Turkey } C k \text { at Olustee } \\
\text { Turkey } C k \text { at Olustee }\end{array}$ & $\begin{array}{l}\text { SED } \\
\text { STR } \\
\text { STR } \\
\text { QWP } \\
\text { MISC }\end{array}$ & $C P$ & & \rfloor & $\begin{array}{r}1666 \\
244 \\
293 \\
293 \\
293\end{array}$ & $\begin{array}{l}1976-78 \\
1905-08 \\
1960-64 \\
1952,54-57,61-63 \\
1987-\end{array}$ \\
\hline $\begin{array}{l}\varnothing 73 \emptyset 11 \emptyset \emptyset \\
6730111 \emptyset \\
\varnothing 736111 \emptyset \\
\varnothing 73 \varnothing 111 \emptyset \\
\varnothing 7301315\end{array}$ & $\begin{array}{l}\text { Turkey Ck at Olustee } \\
\text { Salt Fk Red R nr Elmer } \\
\text { Salt Fk Red R nr Elmer } \\
\text { Salt Fk Red R nr Elmer } \\
\text { N Fk Red R nr Texola }\end{array}$ & $\begin{array}{l}\text { STR } \\
\text { STR } \\
\text { QWP } \\
\text { SED } \\
\text { QWMP }\end{array}$ & $\begin{array}{l}\text { BCNP } \\
\text { S } \\
\text { CNP }\end{array}$ & OBS & $\begin{array}{l}J G \\
J G \\
A \\
A\end{array}$ & $\begin{array}{l}293 \\
1878 \\
1878 \\
1878 \\
1284\end{array}$ & $\begin{array}{l}1987- \\
1979- \\
1978- \\
1978- \\
1977\end{array}$ \\
\hline 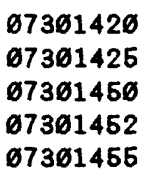 & $\begin{array}{l}\text { Sweetwater Ck } n r \text { Sweetwater } \\
\text { Sweetwater Ck } n r \text { Texas Line } \\
\text { N Fk Red R } n r \text { Erick } \\
\text { Starvation Ck nr Prentiss } \\
\text { Turkey Ck nr Erick }\end{array}$ & $\begin{array}{l}\text { STR } \\
\text { QWP } \\
\text { QWP } \\
\text { LF } \\
\text { CS }\end{array}$ & $\begin{array}{l}C P \\
C O P\end{array}$ & & $J G$ & $\begin{array}{r}1223 . \\
44.5 \\
19.8\end{array}$ & $\begin{array}{l}1986- \\
1953-64 \\
1952,60-63 \\
1964-73 \\
1964-85\end{array}$ \\
\hline $\begin{array}{l}\emptyset 7301455 \\
\emptyset 73 \varnothing 146 \emptyset \\
\emptyset 73 \varnothing 146 \emptyset \\
\emptyset 730148 \emptyset \\
\varnothing 73 \varnothing 1481\end{array}$ & $\begin{array}{l}\text { Turkey Ck nr Erick } \\
\text { Turkey Ck nr Sayre } \\
\text { Turkey Ck nr Sayre } \\
\text { Short Ck nr Sayre } \\
\text { N Fk Red R nr Sayre }\end{array}$ & $\begin{array}{l}\text { QWP } \\
\text { LF } \\
\text { QWP } \\
\text { CS } \\
\text { STR }\end{array}$ & $\begin{array}{l}\mathrm{CP} \\
\mathrm{CP}\end{array}$ & & $H G$ & $\begin{array}{r}19.8 \\
47.5 \\
47.5 \\
9.12 \\
2159 .\end{array}$ & $\begin{array}{l}1953 \\
1953-56,65-73 \\
1953 \\
1964-86 \\
1978-83\end{array}$ \\
\hline 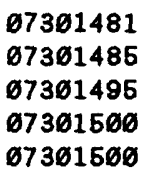 & $\begin{array}{l}\text { N Fk Red R nr Sayre } \\
\text { Spring Ck nr Elk City } \\
\text { Indian Ck nr Carter } \\
\text { N Fk Red R nr Carter } \\
\text { N Fk Red R nr Carter }\end{array}$ & $\begin{array}{l}\text { STGU } \\
\text { CS } \\
\text { CS } \\
\text { STR } \\
\text { QWH }\end{array}$ & CNP & OBS & TQG & $\begin{array}{l}2169 . \\
\quad .93 \\
24.9 \\
2337 . \\
2337 .\end{array}$ & $\begin{array}{l}1984- \\
1968-72 \\
1965-75 \\
1944-62,64- \\
* * 1949,58-63,68-79\end{array}$ \\
\hline
\end{tabular}

* Continuous streamflow records for this period.

* Some records in intervening years.

*** Some records may have been collected previously. 
Table 2.--Station number listing of current and historical gaging stations maintained by the U.S. Geological Survey

September s0, 1987--Continued

[Symbols defined on last page of report]

\begin{tabular}{|c|c|c|c|c|c|c|c|}
\hline $\begin{array}{c}\text { Station } \\
\text { Number }\end{array}$ & Station & $\begin{array}{l}\text { Type } \\
\text { of } \\
\text { Data }\end{array}$ & $\begin{array}{l}\text { Q.W. } \\
\text { Para- } \\
\text { meters }\end{array}$ & $\begin{array}{l}\text { Stage } \\
\text { Source }\end{array}$ & $\begin{array}{l}\text { Coop- } \\
\text { erator }\end{array}$ & $\begin{array}{c}\text { Drainage } \\
\text { Area } \\
(\mathrm{mile})\end{array}$ & Period of Record \\
\hline 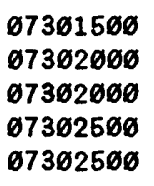 & $\begin{array}{l}\text { N Fk Red R nr Carter } \\
\text { N Fk Red R } n r \text { Granite } \\
\text { N Fk Red R nr Granite } \\
\text { Lake Altus at Lugert } \\
\text { Lake Altus at Lugert }\end{array}$ & $\begin{array}{l}\text { QWMP } \\
\text { STR } \\
\text { SED } \\
\text { RES } \\
\text { QWP }\end{array}$ & CNP & RES & TQG & $\begin{array}{l}2337 . \\
2494 \\
2494 \\
2516 \\
2516\end{array}$ & $\begin{array}{l}1973-79 \\
1963-68,38-44 \\
1905-67 \\
1943- \\
1949,58-52,55-57\end{array}$ \\
\hline $\begin{array}{l}\emptyset 7363060 \\
673630 \varnothing \emptyset \\
\varnothing 7363060 \\
\varnothing 7363395 \\
\varnothing 7363460\end{array}$ & $\begin{array}{l}N \text { Fork Red } R \text { blw Lake Altus } n r \text { Lugert } \\
N \text { Fork Red } R \text { blw Lake Altus } n r \text { Lugert } \\
N \text { Fork Red } R \text { blw Lake Altus } n r \text { Lugert } \\
\text { Elm Fk } N \text { Fk Red R at Salton Crossing } \\
\text { Elm Fk } N \text { Fork Red R nr Carl }\end{array}$ & $\begin{array}{l}\text { STR } \\
\text { QWP } \\
\text { QWMP } \\
\text { QWH } \\
\text { PR }\end{array}$ & $\begin{array}{l}C P \\
C N P \\
C P\end{array}$ & & TQG & $\begin{array}{l}2516 \\
2515 \\
2516 \\
411 \\
416\end{array}$ & $\begin{array}{l}* * 1930-32,64- \\
1963 \\
1973-74 \\
1960-61,73-79 \\
* 1959-79,80-82\end{array}$ \\
\hline $\begin{array}{l}\sigma 73634 \varnothing \emptyset \\
6736346 \emptyset \\
\varnothing 73634 \varnothing \emptyset \\
\varnothing 73634 \varnothing \emptyset \\
\varnothing 7363462\end{array}$ & $\begin{array}{l}\text { Elm Fk } N \text { Fork Red R nr Carl } \\
\text { Elm Fk N Fork Red R nr Carl } \\
\text { Elm Fk N Fork Red R nr Carl } \\
\text { Elm Fk N Fork Red R nr Carl } \\
\text { Fish Ck nr Vinson }\end{array}$ & $\begin{array}{l}\text { QWP } \\
\text { QWMP } \\
\text { SED } \\
\text { STR } \\
\text { PR }\end{array}$ & $\begin{array}{l}\text { CP } \\
\text { CNP } \\
S\end{array}$ & & $J$ & $\begin{array}{l}416 \\
416 \\
416 \\
416 \\
31.5\end{array}$ & $\begin{array}{l}1960-63,68-82 \\
1976-77 \\
1978-79 \\
1959-79 \\
1978-79\end{array}$ \\
\hline $\begin{array}{l}\emptyset 7363462 \\
67363464 \\
67363464 \\
67363466 \\
67363466\end{array}$ & $\begin{array}{l}\text { Fish Ck } n r \text { Vinson } \\
\text { Salt Ck } n r \text { Vinson } \\
\text { Salt Ck } n r \text { Vinson } \\
\text { Elm Fk } N \text { Fk Red } R \text { n Vinson } \\
\text { Elm Fk } N \text { Fk Red R nr Vinson }\end{array}$ & $\begin{array}{l}\text { QWP } \\
\text { PR } \\
\text { QWP } \\
\text { PR } \\
\text { QWP }\end{array}$ & $\begin{array}{l}\mathrm{CP} \\
\mathrm{CP} \\
\mathrm{CP}\end{array}$ & & & $\begin{array}{l}31.6 \\
5.64 \\
5.64 \\
428 . \\
428\end{array}$ & $\begin{array}{l}1978-79 \\
1978-79 \\
1978-79 \\
1978-81 \\
1978-81\end{array}$ \\
\hline 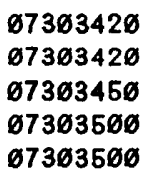 & $\begin{array}{l}\text { Elm Fk N Fk Red R nr Reed } \\
\text { Elm Fk N Fk Red R nr Reed } \\
\text { Deer Ck } n r \text { Plainview } \\
\text { Elm Fk N Fk Red R nr Mangum } \\
\text { Elm Fk N Fk Red R nr Mangum }\end{array}$ & $\begin{array}{l}\text { STR } \\
\text { QWP } \\
\text { CSR } \\
\text { STR } \\
\text { QWH }\end{array}$ & $\mathrm{CP}$ & & & $\begin{array}{l}679 . \\
679 . \\
27.8 \\
838 . \\
838 .\end{array}$ & $\begin{array}{l}1965-67 \\
1978 \\
1964-77 \\
* * 1905-98,65-76 \\
* * 1951,1968-76\end{array}$ \\
\hline $\begin{array}{l}\emptyset 7363506 \\
\varnothing 7364060 \\
\varnothing 7364167 \\
67364299 \\
\varnothing 7364463\end{array}$ & $\begin{array}{l}\text { Elm Fk N Fk Red R nr Mangum } \\
\text { N Fk Red R nr Lugert } \\
\text { Elk Ck nr Elk City } \\
\text { Spring Ck nr Sentinel } \\
\text { E Elk Ck nr Rocky }\end{array}$ & $\begin{array}{l}\text { QWMP } \\
\text { STR } \\
\text { QWP } \\
\text { QWP } \\
\text { QWP }\end{array}$ & $\begin{array}{l}\mathrm{CNP} \\
\mathrm{CP} \\
\mathrm{CP} \\
\mathrm{CP}\end{array}$ & & & $\begin{array}{r}838 \\
3435\end{array}$ & $\begin{array}{l}1976-79 \\
1930-31 \\
196 \emptyset \\
196 \emptyset \\
1961\end{array}$ \\
\hline 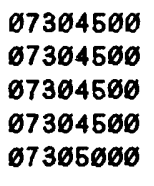 & $\begin{array}{l}\text { Elk Ck nr Hobart } \\
\text { Elk Ck nr Hobart } \\
\text { Elk Ck nr Hobart } \\
\text { Elk Ck nr Hobart } \\
\text { N Fk Red R nr Headrick }\end{array}$ & $\begin{array}{l}\text { STR } \\
\text { QWP } \\
\text { QWMP } \\
\text { QWD } \\
\text { STR }\end{array}$ & $\begin{array}{l}C P \\
C N P \\
P\end{array}$ & OBS & $\begin{array}{l}D G \\
D G \\
D G \\
C\end{array}$ & $\begin{array}{l}549 . \\
549 . \\
549 . \\
549 . \\
4244\end{array}$ & $\begin{array}{l}* * 1904-08,49- \\
* * 1949,54-63,70-87 \\
1975-79 \\
1949-51,58-63,69-87 \\
* * 1905-\varnothing 8,37-\end{array}$ \\
\hline 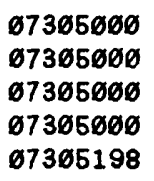 & $\begin{array}{l}\text { N Fk Red R nr Headrick } \\
N \text { Fk Red R nr Headrick } \\
N \text { Fk Red R nr Headrick } \\
\text { N Fk Red R nr Headrick } \\
\text { W Otter Ck at Cold Spring }\end{array}$ & $\begin{array}{l}\text { QWH } \\
\text { QWMP } \\
\text { SED } \\
\text { QWP } \\
\text { QWP }\end{array}$ & $\begin{array}{l}\text { BCNP } \\
\text { CNP } \\
S \\
\text { BCNP } \\
C P\end{array}$ & OBS & $\stackrel{A}{A}$ & $\begin{array}{l}4244 . \\
4244 . \\
4244 . \\
4244 .\end{array}$ & $\begin{array}{l}* * 1951,54-63,68-81 \\
1973-77 \\
* * * 1973- \\
1982- \\
1961\end{array}$ \\
\hline
\end{tabular}

* Continuous streamflow records for this period.

** Some records in intervening years.

*** Some records may have been collected previously. 
Table 2.--Station number listing of current and historical gaging stations maintained by the U.S. Geological Survey

September 30, 1987--Continued

[Symbols defined on last page of report]

\begin{tabular}{|c|c|c|c|c|c|c|c|}
\hline $\begin{array}{l}\text { Station } \\
\text { Number }\end{array}$ & Station & $\begin{array}{l}\text { Type } \\
\text { of } \\
\text { Data }\end{array}$ & $\begin{array}{l}\text { Q.W. } \\
\text { Para- } \\
\text { meters }\end{array}$ & $\begin{array}{l}\text { Stage } \\
\text { Source }\end{array}$ & $\begin{array}{l}\text { Coop- } \\
\text { orator }\end{array}$ & $\begin{array}{c}\text { Drainage } \\
\text { Area } \\
\left(m i l{ }^{2}\right)\end{array}$ & Period of Record \\
\hline $\begin{array}{l}\emptyset 7305362 \\
\emptyset 7365400 \\
\emptyset 7305500 \\
\varnothing 7305500 \\
\emptyset 7306060\end{array}$ & $\begin{array}{l}\text { Glenn Ck nr Cold Spring } \\
\text { Tom Steed Res nr Mountain Park } \\
\text { W Otter Ck at Snyder Lake nr Mt Park } \\
\text { W Otter Ck at Snyder Lake nr Mt Park } \\
\text { Horse Ck nr Mountain Park }\end{array}$ & $\begin{array}{l}\text { QWP } \\
\text { RES } \\
\text { STR } \\
\text { QWP } \\
\text { STR }\end{array}$ & $\mathrm{CP}$ & & $\begin{array}{l}C \\
D G\end{array}$ & $\begin{array}{c}132 . \\
132 \\
11.1\end{array}$ & $\begin{array}{l}1961 \\
1986- \\
1963-68,51- \\
1960 \\
1966\end{array}$ \\
\hline 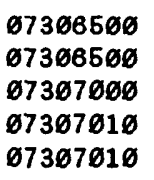 & $\begin{array}{l}\text { Otter Ck at Mt Park } \\
\text { Otter Ck at Mt Park } \\
\text { Dry Fk Otter Ck nr Mountain Park } \\
\text { Otter Ck nr Snyder } \\
\text { Otter Ck nr Snyder }\end{array}$ & $\begin{array}{l}\text { STR } \\
\text { QWP } \\
\text { STR } \\
\text { QWP } \\
\text { STGU }\end{array}$ & $\begin{array}{l}C P \\
C P\end{array}$ & DCP & C & $\begin{array}{l}164 \\
164 \\
12.0\end{array}$ & $\begin{array}{l}1946-51 \\
1952 \\
1965-66 \\
1951,60-63 \\
1984-\end{array}$ \\
\hline 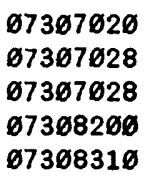 & $\begin{array}{l}\text { Otter Ck nr Tipton } \\
\text { N Fk Red R nr Tipton } \\
\text { N Fk Red R nr Tipton } \\
\text { Pease R nr Vernon TX } \\
\text { Suttle Ck nr Davidson }\end{array}$ & $\begin{array}{l}\text { QWP } \\
\text { STR } \\
\text { QWP } \\
\text { QWP } \\
\text { LF }\end{array}$ & $\begin{array}{l}\mathrm{CP} \\
\mathrm{CP} \\
\mathrm{C}\end{array}$ & OBS & $J$ & $\begin{array}{l}4681 \\
4681 \\
3488 . \\
65.0\end{array}$ & $\begin{array}{l}1960-63 \\
1983- \\
1960 \\
1960 \\
1965-66\end{array}$ \\
\hline $\begin{array}{l}\emptyset 73086 \varnothing \emptyset \\
\varnothing 736889 \emptyset \\
\varnothing 736899 \emptyset \\
\varnothing 73690 \varnothing \emptyset \\
\varnothing 73090 \varnothing \emptyset\end{array}$ & $\begin{array}{l}\text { Red R nr Burkburnett TX } \\
\text { E Cache Ck nr Apache } \\
\text { Lake Ellsworth nr Elgin } \\
\text { E Cache Ck nr Elgin } \\
\text { E Cache Ck nr Elgin }\end{array}$ & $\begin{array}{l}\text { QWMP } \\
\text { QWP } \\
\text { RESU } \\
\text { STR } \\
\text { QWD }\end{array}$ & $\begin{array}{l}\text { CNP } \\
\mathrm{C}\end{array}$ & & UG & $\begin{array}{l}20670 \text {. } \\
249 \text {. } \\
248 \text {. } \\
248 \text {. }\end{array}$ & $\begin{array}{l}1973-77 \\
1951 \\
1964- \\
1956-58 \\
1958,58\end{array}$ \\
\hline $\begin{array}{l}07309600 \\
67369480 \\
67309600 \\
67309950 \\
\varnothing 7310000\end{array}$ & $\begin{array}{l}\text { E Cache Ck nr Elgin } \\
\text { Canyon Ck nr Medicine Park } \\
\text { Lake Lawtonka nr Lawton } \\
\text { Medicine Ck nr Fort Sill } \\
\text { Little Medicine Bluff Ck nr Lawton }\end{array}$ & $\begin{array}{l}\text { QWMP } \\
\text { CS } \\
\text { RESU } \\
\text { QWP } \\
\text { STR }\end{array}$ & CNP & RES & UG & $\begin{array}{l}248 . \\
3.35 \\
93.0 \\
7.06\end{array}$ & $\begin{array}{l}1976-79 \\
1965-75 \\
1955- \\
1951 \\
1913-19\end{array}$ \\
\hline $\begin{array}{l}\varnothing 73165 \varnothing \varnothing \\
\varnothing 7311 \varnothing \varnothing \emptyset \\
\varnothing 73116 \varnothing \varnothing \\
\varnothing 73116 \varnothing \emptyset \\
\varnothing 73112 \varnothing \varnothing\end{array}$ & $\begin{array}{l}\text { Medicine Bluff Ck nr Lawton } \\
\text { E Cache Ck nr Walters } \\
\text { E Cache Ck nr Walters } \\
\text { E Cache Ck nr Walters } \\
\text { Blue Beaver Ck nr Cache }\end{array}$ & $\begin{array}{l}\text { STR } \\
\text { STR } \\
\text { QWP } \\
\text { QWMP } \\
\text { STR }\end{array}$ & $\begin{array}{l}\mathrm{CP} \\
\mathrm{CNP}\end{array}$ & & $\begin{array}{l}\text { UG } \\
\text { UG } \\
\text { A }\end{array}$ & $\begin{array}{l}101 \\
675 \\
675 \\
675 \\
24.6\end{array}$ & $\begin{array}{l}1913-19 \\
1938-68,69- \\
* * 1947-48,58-63,70- \\
1975-79 \\
1964-\end{array}$ \\
\hline $\begin{array}{l}\emptyset 73112 \varnothing \emptyset \\
\varnothing 73112 \varnothing \emptyset \\
\varnothing 731124 \varnothing \\
\varnothing 731124 \varnothing \\
\varnothing 731141 \varnothing\end{array}$ & $\begin{array}{l}\text { Blue Beaver Ck nr Cache } \\
\text { Blue Beaver Ck nr Cache } \\
\text { W Cache Ck nr Cookietown } \\
\text { W Cache Ck nr Cookietown } \\
\text { Red Ck nr Snyder }\end{array}$ & $\begin{array}{l}\text { QWP } \\
\text { SED } \\
\text { LF } \\
\text { QWP } \\
\text { CS }\end{array}$ & $\begin{array}{l}\text { BCNP } \\
S \\
C P\end{array}$ & & $\begin{array}{l}A \\
A\end{array}$ & $\begin{array}{l}24.6 \\
24.6 \\
1112 . \\
1112 . \\
6.12\end{array}$ & $\begin{array}{l}1965- \\
1965- \\
1951-55,65-73 \\
1952-55,60-63 \\
1965-74\end{array}$ \\
\hline $\begin{array}{l}\emptyset 731142 \varnothing \\
\varnothing 73115 \varnothing \emptyset \\
6731150 \varnothing \\
\varnothing 73115 \varnothing \emptyset \\
\varnothing 7311505\end{array}$ & $\begin{array}{l}\text { Deadman Ck Trib at Manitou } \\
\text { Deep Red Run nr Randlett } \\
\text { Deep Red Run nr Randlett } \\
\text { Deep Red Run nr Randlett } \\
\text { Deep Red Run nr Taylor }\end{array}$ & $\begin{array}{l}\text { CS } \\
\text { STR } \\
\text { QWD } \\
\text { QWMP } \\
\text { QWP }\end{array}$ & $\begin{array}{l}C P \\
C N P \\
C P\end{array}$ & & $J G$ & $\begin{array}{l}2.57 \\
617 \\
617 \\
617 \\
1121\end{array}$ & $\begin{array}{l}1965-72 \\
1949- \\
* * 1948,69-70 \\
1975 \\
1959\end{array}$ \\
\hline
\end{tabular}

* Continuous streamflow records for this period.

** Some records in intervening years.

*** Some records may have been collected previously. 
Table 2.--Station number listing of current and historical gaging stations maintained by the U.S. Geological Survey

September s0, 1987--Cont inued

[Symbols defined on last page of report]

\begin{tabular}{|c|c|c|c|c|c|c|c|}
\hline $\begin{array}{c}\text { Station } \\
\text { Number }\end{array}$ & Station Name & $\begin{array}{l}\text { Type } \\
\text { of } \\
\text { Data }\end{array}$ & $\begin{array}{l}\text { Q.W. } \\
\text { Para- } \\
\text { meters }\end{array}$ & $\begin{array}{l}\text { Stage } \\
\text { Source }\end{array}$ & $\begin{array}{l}\text { Coop- } \\
\text { orator }\end{array}$ & $\begin{array}{c}\text { Drainage } \\
\text { Area } \\
\left(m i l e^{2}\right)\end{array}$ & Period of Record \\
\hline $\begin{array}{l}\varnothing 73115 \varnothing 6 \\
\varnothing 731272 \varnothing \\
\varnothing 731276 \varnothing \\
\varnothing 731285 \varnothing \\
\varnothing 731286 \varnothing\end{array}$ & $\begin{array}{l}\text { Deep Red Run nr Taylor } \\
\text { Red R nr Waurika } \\
\text { Beaver Ck nr Lawton } \\
\text { Nine Mile Beaver Ck nr Elgin } \\
\text { Nine Mile Beaver Ck nr Elgin }\end{array}$ & $\begin{array}{l}\text { QWMP } \\
\text { QWMP } \\
\text { QWP } \\
\text { CSR } \\
\text { CS }\end{array}$ & $\begin{array}{l}\text { CNP } \\
\text { CNP } \\
\text { CP }\end{array}$ & & $\begin{array}{l}H G \\
H G\end{array}$ & $\begin{array}{l}1121 . \\
6.29 \\
6.29\end{array}$ & $\begin{array}{l}1976-79 \\
1978-79 \\
1948,61 \\
1964-76 \\
1964-\end{array}$ \\
\hline $\begin{array}{l}\varnothing 7312850 \\
\varnothing 7312960 \\
\varnothing 7313 \varnothing 6 \varnothing \\
673136 \varnothing \emptyset \\
673136 \varnothing 9\end{array}$ & $\begin{array}{l}\text { Nine Mile Beaver Ck nr Elgin } \\
\text { Little Beaver Ck nr Marlow } \\
\text { Little Beaver Ck nr Duncan } \\
\text { Little Beaver Ck nr Duncan } \\
\text { Little Beaver Ck nr Gas City }\end{array}$ & $\begin{array}{l}\text { CS } \\
\text { CS } \\
\text { STR } \\
\text { QWP } \\
\text { QWP }\end{array}$ & $\begin{array}{l}C P \\
C P\end{array}$ & & $H G$ & $\begin{array}{l}6.29 \\
35.40 \\
168 . \\
158 .\end{array}$ & $\begin{array}{l}1964-85 \\
1964-75 \\
1949-63 \\
* * 1948-49,58-63 \\
1961\end{array}$ \\
\hline $\begin{array}{l}\varnothing 7313211 \\
\varnothing 73134 \varnothing \varnothing \\
\varnothing 73135 \varnothing \varnothing \\
\varnothing 731350 \varnothing \\
\varnothing 73135 \varnothing \varnothing\end{array}$ & $\begin{array}{l}\text { Walker Ck nr Corum } \\
\text { Waurika Lake nr Waurika } \\
\text { Beaver Ck nr Waurika } \\
\text { Beaver Ck nr Waurika } \\
\text { Beaver Ck nr Waurika }\end{array}$ & $\begin{array}{l}\text { QWP } \\
\text { RESP } \\
\text { STR } \\
\text { QWP } \\
\text { QWMP }\end{array}$ & $\begin{array}{l}C P \\
C N P\end{array}$ & & $\begin{array}{l}c \\
C\end{array}$ & $\begin{array}{l}562 . \\
563 . \\
663 . \\
563\end{array}$ & $\begin{array}{l}1961 \\
1978- \\
1953- \\
1953-66 \\
1975-79\end{array}$ \\
\hline $\begin{array}{l}\emptyset 7313500 \\
\emptyset 7313533 \\
\varnothing 7313566 \\
\varnothing 73136 \varnothing \emptyset \\
\varnothing 73136 \varnothing \emptyset\end{array}$ & $\begin{array}{l}\text { Beaver Ck nr Waurika } \\
\text { Cow Ck nr Comanche } \\
\text { Dry Ck nr Comanche } \\
\text { Cow Ck at Waurika } \\
\text { Cow Ck at Waurika }\end{array}$ & $\begin{array}{l}\text { SED } \\
\text { QWP } \\
\text { QWP } \\
\text { CS } \\
\text { QWP }\end{array}$ & $\begin{array}{l}\mathrm{S} \\
\mathrm{CP} \\
\mathrm{CP} \\
\mathrm{CP}\end{array}$ & & JG & $\begin{array}{l}563 . \\
193 . \\
193 .\end{array}$ & $\begin{array}{l}1963- \\
1959,61 \\
1961 \\
* 1967-7 \varnothing, 71- \\
1960-63,67-70\end{array}$ \\
\hline $\begin{array}{l}\varnothing 731360 \varnothing \\
\varnothing 73136 \varnothing \emptyset\end{array}$ & $\begin{array}{l}\text { Cow Ck at Waurika } \\
\text { Cow Ck at Waurika }\end{array}$ & $\begin{array}{l}\text { QWMP } \\
\text { STR }\end{array}$ & CNP & & GJ & $\begin{array}{l}193 \\
193\end{array}$ & $\begin{array}{l}1978-79 \\
1967-70\end{array}$ \\
\hline $\begin{array}{l}\emptyset 73137 ø 2 \\
673166 \varnothing 6 \\
\emptyset 7315672\end{array}$ & $\begin{array}{l}\text { Beaver Ck nr Ryan } \\
\text { Red } R \text { nr Terral } \\
\text { W Mud Ck nr Atlee }\end{array}$ & $\begin{array}{l}\text { QWP } \\
\text { QWMP } \\
\text { QWP }\end{array}$ & $\begin{array}{l}C P \\
C N P \\
C P\end{array}$ & & & 28723 & $\begin{array}{l}1960-61 \\
1973-77 \\
1961\end{array}$ \\
\hline $\begin{array}{l}\emptyset 731568 \emptyset \\
\emptyset 7315681 \\
\emptyset 7315681 \\
\varnothing 7315697 \\
\varnothing 73167 \varnothing \varnothing\end{array}$ & $\begin{array}{l}\text { Cottonwood Ck Trib nr Loco } \\
\text { W Mud Ck nr Ringling } \\
\text { W Mud Ck nr Ringling } \\
\text { Mud Ck nr Grady } \\
\text { Mud Ck nr Courtney }\end{array}$ & $\begin{array}{l}\text { CS } \\
\text { QWP } \\
\text { QWP } \\
\text { QWP } \\
\text { STR }\end{array}$ & $\begin{array}{l}C P \\
C P \\
C P\end{array}$ & & HG & 1.74 & $\begin{array}{l}1964-86 \\
1960 \\
1963 \\
1961-62,6 \varnothing-61 \\
1960-\end{array}$ \\
\hline $\begin{array}{l}\emptyset 73157 \emptyset \emptyset \\
\emptyset 73157 \emptyset \emptyset \\
\emptyset 7316873 \\
6731688 \emptyset \\
\emptyset 7315882\end{array}$ & $\begin{array}{l}\text { Mud Ck nr Courtney } \\
\text { Mud Ck nr Courtney } \\
\text { Walnut Bayou nr Oswalt } \\
\text { Demijohn Ck nr Wilson } \\
\text { Simon Ck nr Oswalt }\end{array}$ & $\begin{array}{l}\text { QWP } \\
\text { QWMP } \\
\text { QWP } \\
\text { CS } \\
\text { QWP }\end{array}$ & $\begin{array}{l}\mathrm{CP} \\
\mathrm{CNP} \\
\mathrm{CP} \\
\mathrm{CP}\end{array}$ & & & $\begin{array}{l}572 . \\
572 . \\
\quad 6.74\end{array}$ & $\begin{array}{l}1960,62-63 \\
1975-79 \\
1960 \\
1964-73 \\
1960-61\end{array}$ \\
\hline $\begin{array}{l}\emptyset 7315891 \\
\varnothing 731590 \emptyset \\
\varnothing 731596 \emptyset \\
\varnothing 731590 \varnothing \\
\varnothing 7316 \varnothing \varnothing \emptyset\end{array}$ & $\begin{array}{l}\text { Simon Ck at Pike } \\
\text { Walnut Bayou nr Burneyville } \\
\text { Walnut Bayou nr Burneyville } \\
\text { Walnut Bayou nr Burneyville } \\
\text { Red R nr Gainesville TX }\end{array}$ & $\begin{array}{l}\text { QWP } \\
\text { STR } \\
\text { QWD } \\
\text { QWMP } \\
\text { STRP }\end{array}$ & $\begin{array}{l}\mathrm{CP} \\
\mathrm{CP} \\
\mathrm{CNP}\end{array}$ & BDT & C & $\begin{array}{r}314 . \\
314 \\
314 \\
30782\end{array}$ & $\begin{array}{l}1960-61 \\
1961-64,69-71 \\
1960-62,69-71 \\
1975-79 \\
1936-\end{array}$ \\
\hline
\end{tabular}

* Continuous streamflow records for this period.

** Some records in intervening years.

*** Some records may have been collected previously. 
Table 2.--Station number listing of current and historical gaging stations maintained by the U.S. Geological Survey

September 30, 1987--Continued

[Symbols defined on last page of report]

\begin{tabular}{|c|c|c|c|c|c|c|c|}
\hline $\begin{array}{c}\text { Station } \\
\text { Number }\end{array}$ & Station Name & $\begin{array}{l}\text { Type } \\
\text { of } \\
\text { Data }\end{array}$ & $\begin{array}{l}\text { Q.W. } \\
\text { Para- } \\
\text { meters }\end{array}$ & $\begin{array}{l}\text { Stage } \\
\text { Source }\end{array}$ & $\begin{array}{l}\text { Coop- } \\
\text { erator }\end{array}$ & $\begin{array}{c}\text { Drainage } \\
\text { Area } \\
(\mathrm{mile})\end{array}$ & Period of Record \\
\hline $\begin{array}{l}6731606 \emptyset \\
67316 \varnothing 6 \emptyset \\
67316 \varnothing 7 \emptyset \\
\emptyset 731616 \emptyset \\
6731613 \emptyset\end{array}$ & $\begin{array}{l}\text { Red R nr Gainesville TX } \\
\text { Red R nr Gainesville TX } \\
\text { Hickory Ck nr Marietta } \\
\text { Lake Murray nr Ardmore } \\
\text { Wilson Ck Trib nr McMillan }\end{array}$ & $\begin{array}{l}\text { QWP } \\
\text { QWMP } \\
\text { LF } \\
\text { QWP } \\
\text { CS }\end{array}$ & $\begin{array}{l}\text { COP } \\
\text { CNP } \\
C P\end{array}$ & & & $\begin{array}{l}36782 . \\
36782 \\
116 \\
2.97\end{array}$ & $\begin{array}{l}1953-63 \\
1973-77 \\
1964-73 \\
1949-53,56-57 \\
1965-75\end{array}$ \\
\hline $\begin{array}{l}\emptyset 731614 \emptyset \\
\emptyset 731614 \emptyset \\
\emptyset 731614 \emptyset \\
\emptyset 731636 \emptyset \\
\emptyset 731635 \emptyset\end{array}$ & $\begin{array}{l}\text { Brier Ck nr Powell } \\
\text { Brier Ck nr Powell } \\
\text { Brier Ck nr Powell } \\
\text { Washita R nr Reydon } \\
\text { Washita R nr Reydon }\end{array}$ & $\begin{array}{l}\text { CSR } \\
\text { CS } \\
\text { CS } \\
\text { QWP } \\
\text { QWMP }\end{array}$ & $\begin{array}{l}C P \\
C N P\end{array}$ & & $\begin{array}{l}H G \\
H G \\
H G\end{array}$ & $\begin{array}{l}12.0 \\
12.0 \\
12.0 \\
4.98 \\
4.98\end{array}$ & $\begin{array}{l}1965-76 \\
1965- \\
1965-86 \\
1949,52 \\
1977\end{array}$ \\
\hline $\begin{array}{l}\emptyset 731641 \emptyset \\
\emptyset 73166 \varnothing \emptyset \\
\emptyset 73166 \varnothing \emptyset \\
\emptyset 73165 \emptyset \emptyset \\
\emptyset 73176 \varnothing \emptyset\end{array}$ & $\begin{array}{l}\text { Washita } R \text { Trib nr Crawford } \\
\text { Washita } R \text { nr Cheyenne } \\
\text { Washita } R \mathrm{nr} \text { Cheyenne } \\
\text { Washita } R \mathrm{nr} \text { Cheyenne } \\
\text { Sandstone Ck SWS I } 6 \text { A nr Cheyenne }\end{array}$ & $\begin{array}{l}\text { CS } \\
\text { STR } \\
\text { QWP } \\
\text { QWMP } \\
\text { STR }\end{array}$ & $\begin{array}{l}\text { CNP } \\
\text { CNP }\end{array}$ & OBS & $J G$ & $\begin{array}{l}2.18 \\
794 . \\
794 \\
794 . \\
8.78\end{array}$ & $\begin{array}{l}1965-72 \\
1937- \\
1950-63,60-61,69-73 \\
1973-76 \\
1952-74\end{array}$ \\
\hline 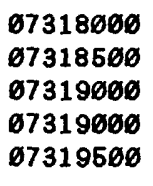 & $\begin{array}{l}\text { Sandstone Ck SWS } 18 \mathrm{nr} \text { Cheyenne } \\
\text { Sandstone Ck SWS } 14 \mathrm{nr} \text { Cheyenne } \\
\text { Sandstone CK SWS } 17 \mathrm{nr} \text { Cheyenne } \\
\text { Sandstone Ck SWS } 17 \mathrm{nr} \text { Cheyenne } \\
\text { Sandstone Ck nr Berlin }\end{array}$ & $\begin{array}{l}\text { STR } \\
\text { STR } \\
\text { STR } \\
\text { QWP } \\
\text { STR }\end{array}$ & $\mathrm{CP}$ & & & $\begin{array}{l}11.6 \\
1.62 \\
16.1 \\
16.1 \\
44.9\end{array}$ & $\begin{array}{l}1953-74 \\
1953-74 \\
1963-74 \\
1969-7 \emptyset \\
1953-72\end{array}$ \\
\hline $\begin{array}{l}\emptyset 731960 \varnothing \\
\emptyset 732060 \varnothing \\
\emptyset 732050 \varnothing \\
\emptyset 732100 \emptyset \\
\varnothing 7321600\end{array}$ & $\begin{array}{l}\text { Sandstone Ck nr Berlin } \\
\text { Sandstone Ck SWS IOA nr EIk City } \\
\text { Sandstone Ck SWS } 6 \text { nr EIk City } \\
\text { Sandstone CK SWS } 5 \text { nr EIk City } \\
\text { Sandstone CK SWS } 3 \mathrm{nr} \text { Elk City }\end{array}$ & $\begin{array}{l}\text { QWP } \\
\text { STR } \\
\text { STR } \\
\text { STR } \\
\text { STR }\end{array}$ & $C P$ & & & $\begin{array}{l}44.9 \\
2.87 \\
6.46 \\
3.89 \\
6.62\end{array}$ & $\begin{array}{l}1958 \\
1952-74 \\
1953-74 \\
1963-74 \\
1963-74\end{array}$ \\
\hline 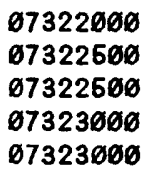 & $\begin{array}{l}\text { Sandstone Ck sWS } 9 \text { nr Elk City } \\
\text { E Br Sandstone Ck nr Elk City } \\
\text { E Br Sandstone Ck nr Elk City } \\
\text { Sandstone Ck nr Cheyenne } \\
\text { Sandstone Ck nr Cheyenne }\end{array}$ & $\begin{array}{l}\text { STR } \\
\text { STR } \\
\text { QWP } \\
\text { STR } \\
\text { QWP }\end{array}$ & $\begin{array}{l}\mathrm{CP} \\
\mathrm{CP}\end{array}$ & & & $\begin{array}{l}3.50 \\
23.0 \\
23.0 \\
87.1 \\
87.1\end{array}$ & $\begin{array}{l}1962-74 \\
1951-72 \\
1968 \\
1951-74 \\
1961-62,67-68\end{array}$ \\
\hline $\begin{array}{l}\emptyset 73235 \emptyset \emptyset \\
\emptyset 7324 \emptyset \emptyset \emptyset \\
\emptyset 7324 \emptyset \emptyset \emptyset \\
\emptyset 732415 \emptyset \\
\emptyset 732419 \emptyset\end{array}$ & $\begin{array}{l}\text { Sandstone CK SWS } 22 \mathrm{nr} \text { Cheyenne } \\
\text { Sandstone CK SWS I nr Cheyenne } \\
\text { Sandstone CK SWS I nr Cheyenne } \\
\text { Washita R nr Moorewood } \\
\text { Quartermaster CK nr Hammon }\end{array}$ & $\begin{array}{l}\text { STR } \\
\text { STR } \\
\text { QWP } \\
\text { QWP } \\
\text { QWP }\end{array}$ & $\begin{array}{l}C O P \\
C P \\
C P\end{array}$ & & & $\begin{array}{l}2.26 \\
5.33 \\
5.33\end{array}$ & $\begin{array}{l}1953-72 \\
1962-74 \\
1969-7 \varnothing \\
197 \varnothing-72 \\
197 \varnothing-71\end{array}$ \\
\hline $\begin{array}{l}\emptyset 73242 \emptyset \emptyset \\
\emptyset 73242 \emptyset \emptyset \\
\emptyset 73242 \varnothing \emptyset \\
\emptyset 73243 \varnothing \emptyset \\
\emptyset 73243 \varnothing \emptyset\end{array}$ & $\begin{array}{l}\text { Washita } R \text { nr Hammon } \\
\text { Washita } R \text { nr Hammon } \\
\text { Washita } R \text { nr Hammon } \\
\text { Foss Reservoir nr Foss } \\
\text { Foss Reservoir nr Foss }\end{array}$ & $\begin{array}{l}\text { STR } \\
\text { QWP } \\
\text { QWMP } \\
\text { RESP } \\
\text { QWP }\end{array}$ & $\begin{array}{l}\text { CNP } \\
\text { CNP } \\
\text { CP }\end{array}$ & $\begin{array}{l}\text { OBS } \\
\text { RES }\end{array}$ & $\begin{array}{l}V G \\
V G \\
J G \\
V G\end{array}$ & $\begin{array}{l}1387 . \\
1387 . \\
1387 . \\
1496 \\
1496\end{array}$ & $\begin{array}{l}1969- \\
1961,70- \\
1978-79 \\
1961- \\
1963-87\end{array}$ \\
\hline
\end{tabular}

* Continuous streamflow records for this period.

* Some records in intervening years.

*** Some records may have been collected previously. 
Table 2.--Station number listing of current and historical gaging stations maintained by the U.S. Geological Survey

September 30, 1987--Continued

[Symbols defined on last page of report]

\begin{tabular}{|c|c|c|c|c|c|c|c|}
\hline $\begin{array}{r}\text { Station } \\
\text { Number }\end{array}$ & Station Name & $\begin{array}{l}\text { Type } \\
\text { of } \\
\text { Data }\end{array}$ & $\begin{array}{l}\text { Q.W. } \\
\text { Para- } \\
\text { meters }\end{array}$ & $\begin{array}{l}\text { Stage } \\
\text { Source }\end{array}$ & $\begin{array}{l}\text { Coop- } \\
\text { erator }\end{array}$ & $\begin{array}{c}\text { Orainage } \\
\text { Area } \\
\left(m i l e^{2}\right)\end{array}$ & Period of Record \\
\hline $\begin{array}{l}\emptyset 73243 \varnothing \emptyset \\
\emptyset 73244 \varnothing \emptyset \\
\emptyset 73244 \varnothing \emptyset \\
\emptyset 73244 \varnothing \emptyset \\
\emptyset 73246 \varnothing \emptyset\end{array}$ & $\begin{array}{l}\text { Foss Reservoir nr Foss } \\
\text { Washita } R n r \text { Foss } \\
\text { Washita } R \text { r Foss } \\
\text { Washita R nr Foss } \\
\text { Barnitz Ck nr Arapaho }\end{array}$ & $\begin{array}{l}\text { QWMP } \\
\text { STR } \\
\text { QWP } \\
\text { QWMP } \\
\text { STR }\end{array}$ & $\begin{array}{l}\text { CNP } \\
\text { CNP } \\
\text { CNP }\end{array}$ & OBS & $\begin{array}{l}\text { VG } \\
\text { VG }\end{array}$ & $\begin{array}{l}1496 . \\
1551 \\
1551 \\
1551 \\
243\end{array}$ & $\begin{array}{l}1976-77 \\
* * 1956-57,61-87 \\
* * 1947-48,78-87 \\
1973-79 \\
1943-64\end{array}$ \\
\hline $\begin{array}{l}\emptyset 732450 \emptyset \\
\varnothing 7325 \varnothing \varnothing \emptyset \\
\varnothing 73250 \varnothing \emptyset \\
\varnothing 73253 \varnothing \emptyset \\
\varnothing 73253 \varnothing \varnothing\end{array}$ & $\begin{array}{l}\text { Barnitz Ck nr Arapaho } \\
\text { Washita } R n r \text { Clinton } \\
\text { Washita } R r \text { Clinton } \\
\text { Rainy Mtn Ck at Mountain View } \\
\text { Rainy Mtn Ck at Mountain View }\end{array}$ & $\begin{array}{l}\text { QWP } \\
\text { STR } \\
\text { QWP } \\
\text { LF } \\
\text { QWP }\end{array}$ & $\begin{array}{l}C P \\
C P \\
C P\end{array}$ & & C & $\begin{array}{r}243 . \\
1977 . \\
1977 . \\
399 . \\
309 .\end{array}$ & $\begin{array}{l}1952,55 \\
1935- \\
1953,58,60-63 \\
1951-56,58-59 \\
1952-56,58\end{array}$ \\
\hline $\begin{array}{l}\varnothing 73254 \varnothing \emptyset \\
\varnothing 73254 \emptyset \emptyset \\
\varnothing 73255 \emptyset \emptyset \\
\varnothing 73255 \varnothing \emptyset \\
\varnothing 732550 \emptyset\end{array}$ & $\begin{array}{l}\text { Stinking Ck nr Carnegie } \\
\text { Stinking Ck nr Carnegie } \\
\text { Washita } R \text { at Carnegie } \\
\text { Washita } R \text { at Carnegie } \\
\text { Washita } R \text { at Carnegie }\end{array}$ & $\begin{array}{l}\text { LF } \\
\text { QWP } \\
\text { STR } \\
\text { QWP } \\
\text { QWMP }\end{array}$ & $\begin{array}{l}\mathrm{CP} \\
\mathrm{CNP} \\
\mathrm{CNP}\end{array}$ & $\begin{array}{l}\text { OBS } \\
\text { OBS } \\
\text { OBS }\end{array}$ & $\begin{array}{l}\text { JG } \\
\text { VG }\end{array}$ & $\begin{array}{l}104 . \\
104 . \\
3129 . \\
3129 . \\
3129 .\end{array}$ & $\begin{array}{l}1951-56,58-61 \\
1952-55,58 \\
1937- \\
1948-87 \\
1973-76\end{array}$ \\
\hline $\begin{array}{l}\emptyset 7325763 \\
\emptyset 73258 \varnothing \emptyset \\
\emptyset 732585 \emptyset \\
\emptyset 732586 \emptyset \\
\varnothing 732590 \emptyset\end{array}$ & $\begin{array}{l}\text { Spring Ck nr Eakly } \\
\text { Cobb Ck nr Eakly } \\
\text { Lake Ck nr Eakly } \\
\text { Willow Ck nr Albert } \\
\text { Ft Cobb Reservoir nr Ft Cobb }\end{array}$ & $\begin{array}{l}\text { QWP } \\
\text { STR } \\
\text { STR } \\
\text { STR } \\
\text { RESP }\end{array}$ & $\mathrm{CP}$ & RES & NG & $\begin{array}{c}132 . \\
52.0 \\
28.0 \\
304 .\end{array}$ & $\begin{array}{l}1961 \\
1968- \\
1969-78 \\
197 \emptyset-78 \\
1959-\end{array}$ \\
\hline $\begin{array}{l}\emptyset 732590 \emptyset \\
\varnothing 7326 \varnothing \emptyset \emptyset \\
\varnothing 7326 \varnothing \emptyset \emptyset \\
\emptyset 73265 \emptyset \emptyset \\
\varnothing 732650 \emptyset\end{array}$ & $\begin{array}{l}\text { Ft Cobb Reservoir nr Ft Cobb } \\
\text { Cobb Ck } n r \text { Ft Cobb } \\
\text { Cobb Ck } n r \text { Ft Cobb } \\
\text { Washita } R \text { at Anadarko } \\
\text { Washita } R \text { at Anadarko }\end{array}$ & $\begin{array}{l}\text { QWP } \\
\text { QWP } \\
\text { STR } \\
\text { STRP } \\
\text { QWD }\end{array}$ & $\begin{array}{l}C P \\
C P\end{array}$ & & $\begin{array}{l}\text { NG } \\
\text { JG }\end{array}$ & $\begin{array}{l}304 . \\
313 . \\
313 . \\
3656 \\
3656 \text {. }\end{array}$ & $\begin{array}{l}1960-62 \\
* * 1947-68,60,63 \\
1939- \\
* 1902-08,63- \\
1952,65-71\end{array}$ \\
\hline $\begin{array}{l}\emptyset 73265 \varnothing \emptyset \\
\varnothing 732672 \emptyset \\
\varnothing 7327 \varnothing \varnothing \emptyset \\
\varnothing 7327 \varnothing \emptyset \emptyset \\
\varnothing 7327 \varnothing 31\end{array}$ & $\begin{array}{l}\text { Washita } R \text { at Anadarko } \\
\text { Tonkawa Ck nr Anadarko } \\
\text { Sugar Ck nr Gracemont } \\
\text { Sugar Ck nr Gracemont } \\
\text { Spring Ck nr Gracemont }\end{array}$ & $\begin{array}{l}\text { QWMP } \\
\text { QWD } \\
\text { STRP } \\
\text { QWP } \\
\text { QWP }\end{array}$ & $\begin{array}{l}\mathrm{CNP} \\
\mathrm{CP} \\
\mathrm{CP} \\
\mathrm{CP}\end{array}$ & & & $\begin{array}{c}3656 . \\
26 . \emptyset \\
208 . \\
208 .\end{array}$ & $\begin{array}{l}1976-79 \\
1968-71 \\
1956-74 \\
1956-69 \\
1961\end{array}$ \\
\hline $\begin{array}{l}\emptyset 73273 ø \emptyset \\
\varnothing 732732 \emptyset \\
\varnothing 732742 \emptyset \\
\varnothing 7327432 \\
\varnothing 7327435\end{array}$ & $\begin{array}{l}\text { Washita } R \text { nr Chickasha } \\
\text { W Salt Ck nr Chickasha } \\
\text { W Bitter Ck nr Tabler } \\
\text { Spring Ck nr Blanchard } \\
\text { Spring Ck nr Tabler }\end{array}$ & $\begin{array}{l}\text { QWP } \\
\text { QWD } \\
\text { QWD } \\
\text { QWP } \\
\text { QWP }\end{array}$ & $\begin{array}{l}C P \\
C O P \\
C P \\
C \\
C\end{array}$ & & & $\begin{array}{c}22.0 \\
60.8 \\
1.19 \\
2.28\end{array}$ & $\begin{array}{l}1952-63,55,58-61 \\
1968-71 \\
* * 1953,61,65-71 \\
1968-71 \\
1988-71\end{array}$ \\
\hline $\begin{array}{l}\emptyset 7327437 \\
6732744 \emptyset \\
\varnothing 7327449 \\
6732749 \emptyset \\
6732749 \emptyset\end{array}$ & $\begin{array}{l}\text { Spring Ck Trib nr Middleberg } \\
\text { E Bitter Ck nr Tabler } \\
\text { McCardo Ck nr Cement } \\
\text { Littlo Washita R nr Ninnekah } \\
\text { Littlo Washita R nr Ninnekah }\end{array}$ & $\begin{array}{l}\text { QWP } \\
\text { QWD } \\
\text { QWP } \\
\text { STRP } \\
\text { QWD }\end{array}$ & $\begin{array}{l}C \\
C P \\
C P \\
C N P\end{array}$ & & JG & $\begin{array}{l}0.76 \\
35.6 \\
208 . \\
208 .\end{array}$ & $\begin{array}{l}1969-71 \\
196 \emptyset-61,68-71 \\
1958-6 \emptyset \\
1963-86 \\
1948-56,68-71\end{array}$ \\
\hline
\end{tabular}

* Continuous streamflow records for this period.

** Some records in intervening years.

*** Some records may have been collected previously. 
Table 2.--Station number listing of current and historical gaging stations maintained by the

U.S. Geological Survey

September 90, 1987--Continued

[Symbols defined on last page of report]

\begin{tabular}{|c|c|c|c|c|c|c|c|}
\hline $\begin{array}{l}\text { Station } \\
\text { Number }\end{array}$ & Station Name & $\begin{array}{l}\text { Type } \\
\text { of } \\
\text { Data }\end{array}$ & $\begin{array}{l}\text { Q.W. } \\
\text { Para- } \\
\text { meters }\end{array}$ & $\begin{array}{l}\text { Stage } \\
\text { Source }\end{array}$ & $\begin{array}{l}\text { Coop- } \\
\text { orator }\end{array}$ & $\begin{array}{c}\text { Drainage } \\
\text { Area } \\
(m i l e)\end{array}$ & Period of Record \\
\hline $\begin{array}{l}\emptyset 73276 \varnothing \emptyset \\
\varnothing 73276 \varnothing \emptyset \\
\varnothing 7328 \varnothing \varnothing \emptyset \\
\varnothing 7328 \varnothing \varnothing \emptyset \\
\varnothing 7328 \varnothing 3 \emptyset\end{array}$ & $\begin{array}{l}\text { Little Washita } R \text { at Ninnekah } \\
\text { Little Washita } R \text { at Ninnekah } \\
\text { Washita } R n r \text { Tabler } \\
\text { Washita } R \text { nr Tabler } \\
\text { Big Dry Ck nr Alex }\end{array}$ & $\begin{array}{l}\text { STR } \\
\text { QWP } \\
\text { STR } \\
\text { QWD } \\
\text { CS }\end{array}$ & $\begin{array}{l}\mathrm{CP} \\
\mathrm{CP}\end{array}$ & & & $\begin{array}{l}227 \\
227 \\
4706 \\
4706 \\
\quad 7.67\end{array}$ & $\begin{array}{l}1952-63 \\
1968-69,61-63 \\
1940-62 \\
1947-62 \\
1961-74\end{array}$ \\
\hline $\begin{array}{l}\emptyset 7328 \emptyset 4 \emptyset \\
\varnothing 7328 \emptyset 7 \emptyset \\
\varnothing 73281 \varnothing \emptyset \\
\varnothing 73281 \varnothing \emptyset \\
\varnothing 7328173\end{array}$ & $\begin{array}{l}\text { Little Dry Ck nr Alex } \\
\text { Winter Ck nr Alex } \\
\text { Washita R at Alex } \\
\text { Washita R at Alex } \\
\text { Criner Ck nr Criner }\end{array}$ & $\begin{array}{l}\text { CS } \\
\text { STRP } \\
\text { STRP } \\
\text { QWD } \\
\text { QWP }\end{array}$ & $\begin{array}{l}C P \\
C P\end{array}$ & OBS & $\begin{array}{l}\text { JG } \\
\text { JG }\end{array}$ & $\begin{array}{l}\emptyset .88 \\
33 . \emptyset \\
4787 . \\
4787 .\end{array}$ & $\begin{array}{l}1961-74 \\
1964-1987 \\
1964-86 \\
1966-71 \\
1961\end{array}$ \\
\hline $\begin{array}{l}\emptyset 73282 \varnothing \emptyset \\
\varnothing 732825 \emptyset \\
\emptyset 73283 \emptyset \emptyset \\
\varnothing 73286 \varnothing \emptyset \\
\varnothing 73286 \varnothing \emptyset\end{array}$ & $\begin{array}{l}\text { Criner Ck nr Payne } \\
\text { Finn Ck nr Payne } \\
\text { Finn Ck nr Story } \\
\text { Washita } R \text { nr Pauls Valley } \\
\text { Washita R nr Pauls Valley }\end{array}$ & $\begin{array}{l}\text { QWP } \\
\text { QWP } \\
\text { QWP } \\
\text { STR } \\
\text { QWP }\end{array}$ & $\begin{array}{l}C P \\
C P \\
C P \\
C O P\end{array}$ & OBS & JG & $\begin{array}{l}67.2 \\
6330 . \\
6330 .\end{array}$ & $\begin{array}{l}1968-60 \\
1961 \\
1961-60 \\
1937- \\
1962-63\end{array}$ \\
\hline 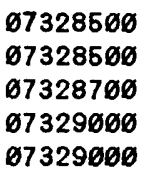 & $\begin{array}{l}\text { Washita } R \text { nr Pauls Valley } \\
\text { Washita } R n r \text { Pauls Valley } \\
\text { Rush Ck nr Rush Springs } \\
\text { Rush Ck at Purdy } \\
\text { Rush Ck at Purdy }\end{array}$ & $\begin{array}{l}\text { QWPM } \\
\text { SED } \\
\text { LF } \\
\text { STR } \\
\text { QWP }\end{array}$ & $\begin{array}{l}\text { CNP } \\
\mathbf{S}\end{array}$ & OBS & JG & $\begin{array}{l}6336 . \\
6336 . \\
146 . \\
146 .\end{array}$ & $\begin{array}{l}1976-79 \\
1976-78 \\
1962 \\
1946-63,82- \\
1947-64,58\end{array}$ \\
\hline $\begin{array}{l}\emptyset 73296 \varnothing \emptyset \\
\varnothing 73296 \varnothing \emptyset \\
\varnothing 73295 \varnothing \emptyset \\
\varnothing 73295 \varnothing \emptyset \\
\varnothing 732965 \emptyset\end{array}$ & $\begin{array}{l}\text { Rush Ck nr Maysville } \\
\text { Rush Ck nr Maysville } \\
\text { Rush Ck nr Maysville } \\
\text { Rush Ck nr Maysville } \\
\text { Rush Ck nr Pauls Valley }\end{array}$ & $\begin{array}{l}\text { STR } \\
\text { QWD } \\
\text { SED } \\
\text { CS } \\
\text { QWP }\end{array}$ & $\begin{array}{l}\mathrm{CP} \\
\mathrm{S} \\
\mathrm{CP}\end{array}$ & & JG & $\begin{array}{l}206 . \\
206 . \\
206 . \\
206 .\end{array}$ & $\begin{array}{l}1953-76 \\
1954-71 \\
1976 \\
1983- \\
1952-53,68-69\end{array}$ \\
\hline $\begin{array}{l}\varnothing 732966 \varnothing \\
\varnothing 73297 \varnothing \varnothing \\
\varnothing 73297 \varnothing \varnothing \\
\varnothing 7329772 \\
\varnothing 7329843\end{array}$ & $\begin{array}{l}\text { Wildhorse Ck nr Hennepin } \\
\text { Wildhorse Ck nr Hoover } \\
\text { Wildhorse Ck nr Hoover } \\
\text { Chigley Sandy Ck nr Davis } \\
\text { Rock Ck } N \text { of Sulphur }\end{array}$ & $\begin{array}{l}\text { QWP } \\
\text { STR } \\
\text { QWD } \\
\text { QWP } \\
\text { QWP }\end{array}$ & $\begin{array}{l}\mathrm{CP} \\
\mathrm{CP} \\
\mathrm{CP} \\
\mathrm{CP}\end{array}$ & & JG & $\begin{array}{l}604 . \\
604 .\end{array}$ & $\begin{array}{l}1949-6 \varnothing \\
1969- \\
1961-69, * 76-71 \\
1956,61 \\
196 \varnothing, 69\end{array}$ \\
\hline $\begin{array}{l}\emptyset 7329847 \\
\emptyset 7329849 \\
\varnothing 7329861 \\
\varnothing 7329853 \\
\varnothing 732986 \emptyset\end{array}$ & $\begin{array}{l}\text { Buffalo Spring at Sulphur } \\
\text { Antelope Springs at Sulphur } \\
\text { Vendome Well Outflow at Sulphur } \\
\text { Rock Ck S of Platt } \mathrm{Nk} \text { Pr Surphur } \\
\text { Honey Ck nr Turner Falls }\end{array}$ & $\begin{array}{l}\text { STR } \\
\text { STR } \\
\text { STR } \\
\text { QWP } \\
\text { QWP }\end{array}$ & $\begin{array}{l}C P \\
C P\end{array}$ & & $\begin{array}{l}b \\
b \\
b\end{array}$ & & $\begin{array}{l}1986- \\
1986- \\
1986- \\
1986 \\
1949-61,54\end{array}$ \\
\hline $\begin{array}{l}\emptyset 732987 \emptyset \\
\varnothing 732987 \emptyset \\
\varnothing 732988 \emptyset \\
\emptyset 732988 \emptyset \\
\varnothing 732989 \emptyset\end{array}$ & $\begin{array}{l}\text { Honey Ck nr Davis } \\
\text { Honey Ck nr Davis } \\
\text { Lawrence Spring nr Drake } \\
\text { Lawrence Spring nr Drake } \\
\text { Arbuckle L nr Dougherty }\end{array}$ & $\begin{array}{l}\text { CS } \\
\text { QWP } \\
\text { QWP } \\
\text { QWP } \\
\text { RES }\end{array}$ & $\begin{array}{l}C P \\
C P \\
C P\end{array}$ & DCP & $H G$ & $\begin{array}{l}18.7 \\
18.7\end{array}$ & $\begin{array}{l}1964-85 \\
1963,66-66 \\
1963 \\
1962,66-56 \\
1986-\end{array}$ \\
\hline
\end{tabular}

* Continuous streamflow records for this period.

** Some records in intervening years.

*** Some records may have been collected previously. 
Table 2.--Station number listing of current and historical gaging stations maintained by the U.S. Geological Survey

September so, 1987--Continued

[Symbols defined on last page of report]

\begin{tabular}{|c|c|c|c|c|c|c|c|}
\hline $\begin{array}{r}\text { Station } \\
\text { Number }\end{array}$ & Station & $\begin{array}{c}\text { Type } \\
\text { of } \\
\text { Data }\end{array}$ & $\begin{array}{l}\text { Q.W. } \\
\text { Para- } \\
\text { meters }\end{array}$ & $\begin{array}{l}\text { Stage } \\
\text { Source }\end{array}$ & $\begin{array}{l}\text { Coop- } \\
\text { erator }\end{array}$ & $\begin{array}{c}\text { Drainage } \\
\text { Area } \\
(m i l e)\end{array}$ & Period of Record \\
\hline $\begin{array}{l}6732996 \varnothing \\
6732996 \varnothing \\
673366 \varnothing \varnothing \\
\varnothing 733656 \varnothing \\
673365 \varnothing \varnothing\end{array}$ & $\begin{array}{l}\text { Rock Ck at Dougherty } \\
\text { Rock Ck at Dougherty } \\
\text { Washita } R \mathrm{nr} \text { Berwyn } \\
\text { Caddo Ck nr Ardmore } \\
\text { Caddo Ck nr Ardmore }\end{array}$ & $\begin{array}{l}\text { STR } \\
\text { QWD } \\
\text { STR } \\
\text { STR } \\
\text { QWP }\end{array}$ & $\mathrm{CP}$ & & & $\begin{array}{r}138 \\
138 \\
6815 \\
298 \\
298\end{array}$ & $\begin{array}{l}1956-67 \\
1951-6 \emptyset \\
1924-26 \\
1936-5 \emptyset \\
1950-51,58\end{array}$ \\
\hline $\begin{array}{l}6733160 \varnothing \\
673316 \varnothing \varnothing \\
6733166 \varnothing \\
673316 \varnothing \emptyset \\
673316 \varnothing \varnothing\end{array}$ & $\begin{array}{l}\text { Washita } R n r \text { Dickson } \\
\text { Washita } R n r \text { Dickson } \\
\text { Washita } R n r \text { Dickson } \\
\text { Washita } R n r \text { Dickson } \\
\text { Washita } R n r \text { Dickson }\end{array}$ & $\begin{array}{l}\text { STR } \\
\text { QWD } \\
\text { QWMP } \\
\text { SED } \\
\text { QWP }\end{array}$ & $\begin{array}{l}\text { BCNP } \\
\text { CNP } \\
S \\
\text { BCNP }\end{array}$ & OBS & $\begin{array}{l}C A \\
A\end{array}$ & $\begin{array}{l}7202 . \\
7202 \\
7202 \\
7202 \\
7202\end{array}$ & $\begin{array}{l}1928- \\
1944-81 \\
1973-77 \\
* * * 1973- \\
1982-\end{array}$ \\
\hline $\begin{array}{l}\emptyset 733126 \varnothing \\
\varnothing 733126 \varnothing \\
\varnothing 733125 \emptyset \\
\varnothing 733125 \emptyset \\
\varnothing 733136 \emptyset\end{array}$ & $\begin{array}{l}\text { Mill Ck nr Mill Creek } \\
\text { Mill Ck nr Mill Creok } \\
\text { Mill Ck nr Ravia } \\
\text { Mill Ck nr Ravia } \\
\text { Pennington Ck nr Reagan }\end{array}$ & $\begin{array}{l}\text { LF } \\
\text { QWP } \\
\text { STR } \\
\text { QWP } \\
\text { LF }\end{array}$ & $\mathrm{CP}$ & & & $\begin{array}{l}46.4 \\
46.4 \\
89.2 \\
89.2 \\
66.7\end{array}$ & $\begin{array}{l}1952-55,58-71 \\
1952-55,60 \\
1969-71 \\
1969 \\
1951-56,58-73\end{array}$ \\
\hline $\begin{array}{l}\emptyset 733136 \varnothing \\
673314 \varnothing 1 \\
\varnothing 733141 \emptyset \\
\varnothing 73315 \varnothing \emptyset \\
\varnothing 73315 \emptyset \emptyset\end{array}$ & $\begin{array}{l}\text { Pennington Ck nr Reagan } \\
\text { Butcher Pen Ck nr Tishomingo } \\
\text { Buzzard Ck nr Reagan } \\
\text { Lake Texoma } n r \text { Denison TX } \\
\text { Lake Texoma } n r \text { Denison TX }\end{array}$ & $\begin{array}{l}\text { QWP } \\
\text { QWP } \\
\text { CS } \\
\text { RESP } \\
\text { QWP }\end{array}$ & $\begin{array}{l}C P \\
C P\end{array}$ & RES & C & $\begin{array}{l}65.7 \\
\quad 4.30 \\
39719 . \\
39719 .\end{array}$ & $\begin{array}{l}1951-56,58-59 \\
1961 \\
1965-76 \\
1942- \\
1949-51\end{array}$ \\
\hline $\begin{array}{l}6733166 \emptyset \\
673316 \varnothing \emptyset \\
6733200 \emptyset \\
6733260 \emptyset \\
6733267 \emptyset\end{array}$ & $\begin{array}{l}\text { Red } R \text { at Denison Dam } n r \text { Denison TX } \\
\text { Red } R \text { at Denison Dam } n r \text { Denison TX } \\
\text { Red } R n r \text { Colbert } \\
\text { Red } R n r \text { Colbert } \\
\text { Rock Ck } n r \text { Achille }\end{array}$ & $\begin{array}{l}\text { STRP } \\
\text { QWMP } \\
\text { STR } \\
\text { QWP } \\
\text { CS }\end{array}$ & $\begin{array}{l}\mathrm{CP} \\
\mathrm{CP}\end{array}$ & & c & $\begin{array}{l}39720 . \\
39720 \\
39777 \\
39777 . \\
\text { 0. }\end{array}$ & $\begin{array}{l}1961- \\
1973-77 \\
1924-61 \\
1960-61 \\
1965-74\end{array}$ \\
\hline $\begin{array}{l}6733225 \emptyset \\
6733236 \emptyset \\
6733239 \emptyset \\
673324 \varnothing \emptyset \\
6733246 \emptyset\end{array}$ & $\begin{array}{l}\text { Island Bayou nr Albany } \\
\text { Blue } R \text { at Connerville } \\
\text { Blue } R \text { nr Connerville } \\
\text { Blue } R \text { at Milburn } \\
\text { Blue R at Milburn }\end{array}$ & $\begin{array}{l}\text { LF } \\
\text { QWP } \\
\text { STR } \\
\text { STR } \\
\text { QWP }\end{array}$ & $\mathrm{CP}$ & & JG & $\begin{array}{l}132 . \\
162 . \\
203 . \\
203 .\end{array}$ & $\begin{array}{l}1965-73 \\
1951-67,62 \\
1977-79 \\
1965-87 \\
1966-60\end{array}$ \\
\hline $\begin{array}{l}\varnothing 733246 \emptyset \\
6733247 \emptyset \\
\varnothing 73325 \varnothing \emptyset \\
\varnothing 73325 \varnothing \emptyset \\
\varnothing 73325 \varnothing \emptyset\end{array}$ & $\begin{array}{l}\text { Blue } R \text { at Armstrong } \\
\text { Chuckwa Ck nr Durant } \\
\text { Blue } R n r \text { Blue } \\
\text { Blue } R n \text { Blue } \\
\text { Blue } R \mathrm{nr} \text { Blue }\end{array}$ & $\begin{array}{l}\text { QWMP } \\
\text { QWP } \\
\text { STRP } \\
\text { QWD } \\
\text { QWMP }\end{array}$ & $\begin{array}{l}\text { CNP } \\
C P \\
C P \\
C N P\end{array}$ & & C & $\begin{array}{l}224 . \\
476 \\
476 \\
476\end{array}$ & $\begin{array}{l}1977 \\
1963 \\
1936- \\
1951-58,60-63 \\
1973-79\end{array}$ \\
\hline $\begin{array}{l}\emptyset 73327 \varnothing \emptyset \\
\varnothing 733276 \emptyset \\
673328 \varnothing \emptyset \\
\varnothing 733296 \emptyset \\
\varnothing 733296 \emptyset\end{array}$ & $\begin{array}{l}\text { Muddy Boggy Ck nr Parker } \\
\text { Muddy Boggy Ck nr Coalgate } \\
\text { Caney Boggy Ck nr Ashland } \\
\text { Coal Ck nr Lehigh } \\
\text { Coal Ck nr Lehigh }\end{array}$ & $\begin{array}{l}\text { LF } \\
\text { QWP } \\
\text { QWD } \\
\text { STR } \\
\text { QWH }\end{array}$ & $\begin{array}{l}\text { CP } \\
\text { CNP } \\
\text { CNOP }\end{array}$ & & & $\begin{array}{l}174 . \\
49.0 \\
8.10 \\
8.10\end{array}$ & $\begin{array}{l}1958-73 \\
1962 \\
1972-75 \\
1978-81 \\
1977-81\end{array}$ \\
\hline
\end{tabular}

* Continuous streamflow records for this period.

** Some records in intervening years.

*** Some records may have been collected previously. 
Table 2.--Station number listing of current and historical gaging stations maintained by the U.S. Geological Survey

September 30 , 1987--Continued

[Symbols defined on last page of report]

\begin{tabular}{|c|c|c|c|c|c|c|c|}
\hline $\begin{array}{l}\text { Station } \\
\text { Number }\end{array}$ & Station & $\begin{array}{l}\text { Type } \\
\text { of } \\
\text { Data }\end{array}$ & $\begin{array}{l}\text { Q.W. } \\
\text { Para- } \\
\text { meters }\end{array}$ & $\begin{array}{l}\text { Stage } \\
\text { Source }\end{array}$ & $\begin{array}{l}\text { Coop- } \\
\text { orator }\end{array}$ & $\begin{array}{c}\text { Drainage } \\
\text { Area } \\
\left(m i l e^{2}\right)\end{array}$ & Period of Record \\
\hline $\begin{array}{l}\emptyset 733290 \emptyset \\
\varnothing 733295 \emptyset \\
\emptyset 733295 \emptyset \\
\varnothing 733295 \emptyset \\
\emptyset 73330 \varnothing \emptyset\end{array}$ & $\begin{array}{l}\text { Coal Ck nr Lohigh } \\
\text { Muddy Boggy Ck at Atoka } \\
\text { Muddy Boggy Ck at Atoka } \\
\text { Muddy Boggy Ck at Atoka } \\
\mathrm{N} \text { Boggy Ck nr Stringtown }\end{array}$ & $\begin{array}{l}\text { SED } \\
\text { STR } \\
\text { SED } \\
\text { QWP } \\
\text { STR }\end{array}$ & $\begin{array}{l}S \\
S \\
\text { CNP }\end{array}$ & & & $\begin{array}{l}8.16 \\
445 . \\
446 . \\
445 \\
136\end{array}$ & $\begin{array}{l}1978-81 \\
1978-81 \\
1978-81 \\
1978-81 \\
1956-59\end{array}$ \\
\hline $\begin{array}{l}\varnothing 733300 \varnothing \\
6733333 \varnothing \\
\varnothing 73335 \varnothing \varnothing \\
\varnothing 73335 \varnothing \varnothing \\
\varnothing 73335 \varnothing \varnothing\end{array}$ & $\begin{array}{l}\text { N Boggy Ck nr Stringtown } \\
\text { Chickasaw Ck Trib nr Stringtown } \\
\text { Chickasaw Ck nr Stringtown } \\
\text { Chickasaw Ck nr Stringtown } \\
\text { Chickasaw Ck nr Stringtown }\end{array}$ & $\begin{array}{l}\text { QWP } \\
\text { CS } \\
\text { CS } \\
\text { QWP } \\
\text { STR }\end{array}$ & $\begin{array}{r}\mathrm{CP} \\
\mathrm{CP} \\
\mathrm{CNP}\end{array}$ & & GJ & $\begin{array}{l}136 . \\
3.19 \\
32.7 \\
32.7 \\
32.7\end{array}$ & $\begin{array}{l}1956-59 \\
1965-72 \\
* 1955-68,69-75 \\
1955-58,6 \varnothing \\
1955-68\end{array}$ \\
\hline $\begin{array}{l}\emptyset 73338 \varnothing \emptyset \\
\emptyset 73338 \varnothing \emptyset \\
\emptyset 733391 \varnothing \\
\varnothing 733391 \emptyset \\
\varnothing 733391 \varnothing\end{array}$ & $\begin{array}{l}\text { McGee Ck nr Stringtown } \\
\text { McGee Ck nr Stringtown } \\
\text { McGee Ck nr Farris } \\
\text { McGee Ck nr Farris } \\
\text { McGee Ck nr Farris }\end{array}$ & $\begin{array}{l}\text { CS } \\
\text { QWP } \\
\text { STR } \\
\text { QWH } \\
\text { QWP }\end{array}$ & $\begin{array}{l}\mathrm{CP} \\
\mathrm{CP} \\
\mathrm{CNP}\end{array}$ & & & $\begin{array}{l}86.6 \\
86.6 \\
176 . \\
176 \\
176\end{array}$ & $\begin{array}{l}* 1956-68,69-76 \\
1956-58 \\
1976-82 \\
1976-82 \\
1976-82\end{array}$ \\
\hline $\begin{array}{l}\emptyset 733391 \emptyset \\
\varnothing 7334 \varnothing \varnothing \varnothing \\
\emptyset 7334 \varnothing \varnothing \varnothing \\
\varnothing 7334 \varnothing \varnothing \varnothing \\
\varnothing 733420 \varnothing\end{array}$ & $\begin{array}{l}\text { McGee Ck nr Farris } \\
\text { Muddy Boggy Ck nr Farris } \\
\text { Muddy Boggy Ck nr Farris } \\
\text { Muddy Boggy Ck nr Farris } \\
\text { Byrds Mill Spring nr Fittstown }\end{array}$ & $\begin{array}{l}\text { SEO } \\
\text { STRP } \\
\text { QWP } \\
\text { QWMP } \\
\text { STR }\end{array}$ & $\begin{array}{l}S \\
C P \\
C N P\end{array}$ & & RG & $\begin{array}{l}176 . \\
1087 \\
1087 \\
1087\end{array}$ & $\begin{array}{l}1978-81 \\
1937- \\
1948,50-58,62-64 \\
1973-79 \\
1959-\end{array}$ \\
\hline $\begin{array}{l}\emptyset 73342 \emptyset \emptyset \\
\emptyset 73344 \emptyset \emptyset \\
\emptyset 73344 \emptyset \emptyset \\
\emptyset 733442 \emptyset \\
\varnothing 733442 \emptyset\end{array}$ & $\begin{array}{l}\text { Byrds Mill Spring nr Fittstown } \\
\text { Clear Boggy Ck nr Tupelo } \\
\text { Clear Boggy Ck nr Tupelo } \\
\text { Leader Ck at Tupelo } \\
\text { Leader Ck at Tupelo }\end{array}$ & $\begin{array}{l}\text { QWP } \\
\text { LF } \\
\text { QWP } \\
\text { LF } \\
\text { QWP }\end{array}$ & $\begin{array}{l}\mathrm{COP} \\
\mathrm{CP}\end{array}$ & & & $\begin{array}{l}248 . \\
248 . \\
64.3 \\
64.3\end{array}$ & $\begin{array}{l}1953,55-56 \\
1958-73 \\
1958,60,62 \\
1958-73 \\
1958,60\end{array}$ \\
\hline $\begin{array}{l}\emptyset 733444 \varnothing \\
\varnothing 733444 \varnothing \\
\varnothing 73345 \varnothing \varnothing \\
\varnothing 733486 \emptyset \\
\varnothing 733500 \emptyset\end{array}$ & $\begin{array}{l}\text { Delaware Ck nr Wapanucka } \\
\text { Delaware Ck nr Wapanucka } \\
\text { Clear Boggy Ck nr Wapanucka } \\
\text { Clear Boggy Ck abv Caney Ck nr Caney } \\
\text { Clear Boggy Ck nr Caney }\end{array}$ & $\begin{array}{l}\text { STR } \\
\text { QWP } \\
\text { STR } \\
\text { QWMP } \\
\text { STRP }\end{array}$ & $C P$ & & $c$ & $\begin{array}{l}45.8 \\
45.8 \\
516 . \\
720 .\end{array}$ & $\begin{array}{l}1958-73 \\
1958,60 \\
194 \varnothing-43 \\
1975-78 \\
1942-\end{array}$ \\
\hline $\begin{array}{l}\emptyset 7335 \varnothing \varnothing \varnothing \\
\varnothing 7335 \varnothing \varnothing \varnothing \\
\varnothing 73353 \varnothing \emptyset \\
\varnothing 73353 \varnothing \varnothing \\
\varnothing 733531 \varnothing\end{array}$ & $\begin{array}{l}\text { Clear Boggy Ck nr Caney } \\
\text { Clear Boggy Ck nr Caney } \\
\text { Muddy Boggy Ck nr Unger } \\
\text { Muddy Boggy Ck nr Unger } \\
\text { Rock Ck nr Boswell }\end{array}$ & $\begin{array}{l}\text { QWP } \\
\text { QWMP } \\
\text { STR } \\
\text { QWP } \\
\text { CS }\end{array}$ & $\begin{array}{l}\text { CP } \\
\text { CNP } \\
C P\end{array}$ & OBS & $\begin{array}{l}\text { JG } \\
\mathrm{HG}\end{array}$ & $\begin{array}{l}720 . \\
720 . \\
\\
\\
0.94\end{array}$ & $\begin{array}{l}1952-75 \\
1975-79 \\
1982- \\
1962 \\
1965-85\end{array}$ \\
\hline $\begin{array}{l}\emptyset 733532 \varnothing \\
\varnothing 73355 \varnothing \emptyset \\
\varnothing 73355 \varnothing \emptyset \\
\varnothing 73356 \varnothing \varnothing \\
\varnothing 73357 \varnothing \varnothing\end{array}$ & $\begin{array}{l}\text { Bokchito Ck nr Soper } \\
\text { Red R at Arthur City TX } \\
\text { Red } R \text { at Arthur City TX } \\
\text { Red } R \text { at Arthur City TX } \\
\text { Kiamichi R nr Big Cedar }\end{array}$ & $\begin{array}{l}\text { CS } \\
\text { STRP } \\
\text { QWP } \\
\text { QWMP } \\
\text { STR }\end{array}$ & $\begin{array}{l}C P \\
C N P\end{array}$ & DAR & $c$ & $\begin{array}{l}16.6 \\
44531 \\
44531 \\
44531 . \\
40.1\end{array}$ & $\begin{array}{l}1965-75 \\
* * 1936- \\
1960-63 \\
1975-79 \\
1965-\end{array}$ \\
\hline
\end{tabular}

* Continuous streamflow records for this period.

** Some records in intervening years.

*** Some records may have been collected previously. 
Table 2.--Station number listing of current and historical gaging stations maintained by the U.S. Geological Survey September so, 1987--Continued

[Symbols defined on last page of report]

\begin{tabular}{|c|c|c|c|c|c|c|c|}
\hline $\begin{array}{c}\text { Station } \\
\text { Number }\end{array}$ & Station Name & $\begin{array}{l}\text { Type } \\
\text { of } \\
\text { Data }\end{array}$ & $\begin{array}{l}\text { Q.W. } \\
\text { Para- } \\
\text { meters }\end{array}$ & $\begin{array}{l}\text { Stage } \\
\text { Source }\end{array}$ & $\begin{array}{l}\text { Coop- } \\
\text { orator }\end{array}$ & $\begin{array}{c}\text { Drainage } \\
\text { Area } \\
(\mathrm{mile})\end{array}$ & Period of Record \\
\hline 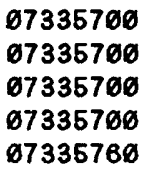 & $\begin{array}{l}\text { Kiamichi R nr Big Cedar } \\
\text { Kiamichi R nr Big Cedar } \\
\text { Kiamichi R nr Big Cedar } \\
\text { Kiamichi R nr Big Cedar } \\
\text { Kiamichi R Trib nr Albion }\end{array}$ & $\begin{array}{l}\text { QWP } \\
\text { QWMP } \\
\text { SED } \\
\text { QWP } \\
\text { CS }\end{array}$ & $\begin{array}{l}\text { BCNPR } \\
\text { CNP } \\
\text { S } \\
\text { BCNPRS }\end{array}$ & & $\begin{array}{l}A \\
C A \\
A\end{array}$ & $\begin{array}{l}40.1 \\
40.1 \\
40.1 \\
40.1 \\
1.50\end{array}$ & $\begin{array}{l}1966- \\
1973-79 \\
1974- \\
1982- \\
1965-72\end{array}$ \\
\hline $\begin{array}{l}\emptyset 7335775 \\
\varnothing 7335785 \\
\emptyset 7335796 \\
\emptyset 733579 \emptyset \\
\varnothing 733590 \emptyset\end{array}$ & $\begin{array}{l}\text { Sardis Lake at Clayton } \\
\text { Jacks Fk Ck at Sardis Lake } \\
\text { Kiamichi } R \mathrm{nr} \text { Clayton } \\
\text { Kiamichi } R \mathrm{nr} \text { Clayton } \\
\text { Buck Ck } n r \text { Moyers }\end{array}$ & $\begin{array}{l}\text { RESP } \\
\text { STRP } \\
\text { QWMP } \\
\text { STRP } \\
\text { QWP }\end{array}$ & $\begin{array}{l}\text { CNP } \\
C P\end{array}$ & & $\begin{array}{l}c \\
c \\
c\end{array}$ & $\begin{array}{l}275 \\
275 \\
708 \\
708 \\
100\end{array}$ & $\begin{array}{l}1983- \\
1984- \\
1977 \\
1980- \\
1956-57,60\end{array}$ \\
\hline 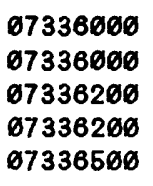 & $\begin{array}{l}\text { Tenmile Ck nr Miller } \\
\text { Tenmile Ck nr Miller } \\
\text { Kiamichi R nr Antlers } \\
\text { Kiamichi R nr Antlers } \\
\text { Kiamichi R nr Belzoni }\end{array}$ & $\begin{array}{l}\text { QWP } \\
\text { CS } \\
\text { STRP } \\
\text { QWMP } \\
\text { STRP }\end{array}$ & CNP & DAR & $\begin{array}{l}\mathrm{HG} \\
\mathrm{C}\end{array}$ & $\begin{array}{l}68.0 \\
68.0 \\
1138 . \\
1138 \\
1423\end{array}$ & $\begin{array}{l}1956-58 \\
1957-70,71-86 \\
1972- \\
1975-79 \\
1926-72\end{array}$ \\
\hline $\begin{array}{l}\varnothing 73365 \varnothing \varnothing \\
\varnothing 733652 \varnothing \\
\varnothing 73366 \varnothing \varnothing \\
\varnothing 73367 \varnothing \varnothing \\
\varnothing 73367 \varnothing \varnothing 6\end{array}$ & $\begin{array}{l}\text { Kiamichi } R \text { nr Belzoni } \\
\text { Frazier Ck nr Oleta } \\
\text { Hugo Lake nr Hugo } \\
\text { Kiamichi } R \text { nr Sawyer } \\
\text { Kiamichi R nr Sawyer }\end{array}$ & $\begin{array}{l}\text { QWD } \\
\text { CS } \\
\text { RESP } \\
\text { QWP } \\
\text { QWMP }\end{array}$ & $\begin{array}{l}\mathrm{CP} \\
\mathrm{CP} \\
\mathrm{CNP}\end{array}$ & & $\begin{array}{l}\mathrm{HG} \\
\mathrm{C}\end{array}$ & $\begin{array}{c}1423 \\
19.4 \\
1709 .\end{array}$ & $\begin{array}{l}1948-54,62-63 \\
1964-86 \\
1974- \\
1962 \\
1978-79\end{array}$ \\
\hline $\begin{array}{l}\emptyset 733671 \varnothing \\
6733673 \varnothing \\
\varnothing 733678 \varnothing \\
\varnothing 733678 \emptyset \\
\varnothing 7336785\end{array}$ & $\begin{array}{l}\text { Rock Ck nr Sawyer } \\
\text { Red } R \text { nr Valliant } \\
\text { Red } R \text { nr Millerton } \\
\text { Perry Ck nr Idabel } \\
\text { Bokchito Ck nr Garvin }\end{array}$ & $\begin{array}{l}\text { CS } \\
\text { QWP } \\
\text { QWP } \\
\text { CS } \\
\text { CSR }\end{array}$ & $\begin{array}{l}\text { CNP } \\
\text { CNP }\end{array}$ & & & $\begin{array}{l}3.39 \\
46730 . \\
46930 . \\
7.53 \\
2.96\end{array}$ & $\begin{array}{l}1964-74 \\
1971-76 \\
1971-76 \\
1965-73 \\
1965-75\end{array}$ \\
\hline $\begin{array}{l}\varnothing 733682 \emptyset \\
\varnothing 733685 \emptyset \\
\varnothing 7337 \varnothing \varnothing \varnothing \\
6733710 \varnothing \\
\varnothing 733715 \emptyset\end{array}$ & $\begin{array}{l}\text { Red } R \text { nr De Kalb TX } \\
\text { Red } R \text { nr New Boston TX } \\
\text { Red } R \text { at Index AR } \\
\text { Little } R \text { nr Cloudy } \\
\text { Little R nr Alikchi }\end{array}$ & $\begin{array}{l}\text { QWMP } \\
\text { QWP } \\
\text { QWD } \\
\text { QWMP } \\
\text { QWP }\end{array}$ & $\begin{array}{l}\text { CNP } \\
C \\
C \\
\text { CNP } \\
\text { CP }\end{array}$ & & & $\begin{array}{r}47348 \\
4863 \emptyset \\
324\end{array}$ & $\begin{array}{l}1973-77 \\
1961-63 \\
1980-63 \\
1975-79 \\
1953\end{array}$ \\
\hline $\begin{array}{l}\varnothing 733720 \varnothing \\
\varnothing 733722 \emptyset \\
\varnothing 733725 \varnothing \\
\varnothing 733736 \varnothing \\
6733750 \varnothing\end{array}$ & $\begin{array}{l}\text { Little R nr Ringold } \\
\text { Big Br nr Ringold } \\
\text { Little R ab Pine Ck Lake nr Wright Cty } \\
\text { Pine Ck Lakenr Wright City } \\
\text { Little R nr Wright City }\end{array}$ & $\begin{array}{l}\text { QWP } \\
\text { CS } \\
\text { QWMP } \\
\text { RESP } \\
\text { STRP }\end{array}$ & $\begin{array}{l}\mathrm{CP} \\
\mathrm{CNP}\end{array}$ & RES & $\begin{array}{l}C \\
C\end{array}$ & $\begin{array}{l}1.99 \\
635 . \\
645 .\end{array}$ & $\begin{array}{l}1962 \\
1964-74 \\
1976-77 \\
1969- \\
1929-31,44-\end{array}$ \\
\hline $\begin{array}{l}\emptyset 7337500 \\
0733750 \varnothing \\
\varnothing 7337650 \\
\emptyset 7337900 \\
\varnothing 733790 \varnothing\end{array}$ & $\begin{array}{l}\text { Little R nr Wright City } \\
\text { Little R nr Wright City } \\
\text { Little R nr Millerton } \\
\text { Glover Ck } n r \text { Glover } \\
\text { Glover Ck } n r \text { Glover }\end{array}$ & $\begin{array}{l}\text { QWP } \\
\text { QWMP } \\
\text { QWP } \\
\text { STRP } \\
\text { QWP }\end{array}$ & $\begin{array}{l}\mathrm{CP} \\
\mathrm{CNP} \\
\mathrm{CPS} \\
\mathrm{CP}\end{array}$ & DAR & C & $\begin{array}{l}645 . \\
645 . \\
315 . \\
315 .\end{array}$ & $\begin{array}{l}1953 \\
1975-76 \\
1953 \\
1961- \\
1949,53,62-63\end{array}$ \\
\hline
\end{tabular}

* Continuous streamflow records for this period.

* Some records in intervening years.

*** Some records may have been collected previously. 
Table 2.--Station number listing of current and historical gaging stations maintained by the

U.S. Geological Survey

September s0, 1987--Continued

[Symbols defined on last page of report]

\begin{tabular}{|c|c|c|c|c|c|c|c|}
\hline $\begin{array}{l}\text { Station } \\
\text { Number }\end{array}$ & Station & $\begin{array}{l}\text { Type } \\
\text { of } \\
\text { Data }\end{array}$ & $\begin{array}{l}\text { Q.W. } \\
\text { Para- } \\
\text { meters }\end{array}$ & $\begin{array}{l}\text { Stage } \\
\text { Source }\end{array}$ & $\begin{array}{l}\text { Coop- } \\
\text { erator }\end{array}$ & $\begin{array}{c}\text { Drainage } \\
\text { Area } \\
(m i l e)\end{array}$ & Period of Record \\
\hline $\begin{array}{l}\emptyset 733790 \emptyset \\
6733792 \emptyset \\
\varnothing 733795 \emptyset \\
67338 \varnothing \varnothing \emptyset \\
\varnothing 73385 \varnothing \emptyset\end{array}$ & $\begin{array}{l}\text { Glover Ck } n r \text { Glover } \\
\text { Fifteen Ck } n r \text { Glover } \\
\text { Little } R n \text { Garvin } \\
\text { Little } n r \text { Idabel } \\
\text { Little R blw Lukfata } C k n r \text { Idabel }\end{array}$ & $\begin{array}{l}\text { QWMP } \\
\text { CS } \\
\text { QWP } \\
\text { STRP } \\
\text { STRP }\end{array}$ & $\begin{array}{l}\text { CNP } \\
\mathrm{CP}\end{array}$ & DAR & $C$ & $\begin{array}{l}316 . \\
1.23 \\
1173 . \\
1226\end{array}$ & $\begin{array}{l}1975-79 \\
1967-73 \\
1953 \\
1930-46 \\
1946-\end{array}$ \\
\hline $\begin{array}{l}\emptyset 73385 \varnothing \emptyset \\
673385 \varnothing \emptyset \\
\varnothing 733852 \emptyset \\
\varnothing 733852 \emptyset \\
\emptyset 733878 \emptyset\end{array}$ & $\begin{array}{l}\text { Little R blw Lukfata Ck nr Idabel } \\
\text { Little R blw Lukfata Ck nr Idabel } \\
\text { Yanubbee Ck nr Broken Bow } \\
\text { Yanubbee Ck nr Broken Bow } \\
\text { Mountain Fk Trib nr Smithville }\end{array}$ & $\begin{array}{l}\text { QWP } \\
\text { QWMP } \\
\text { CSR } \\
\text { CS } \\
\text { CS }\end{array}$ & $\begin{array}{l}\text { COP } \\
\text { CNP }\end{array}$ & & $\begin{array}{l}H G \\
H G \\
H G\end{array}$ & $\begin{array}{l}1226 \\
1226 \\
9.16 \\
9.10 \\
\emptyset .85\end{array}$ & $\begin{array}{l}1948-64,61-63,69-73 \\
* * 1947-54,76-79 \\
1964-76 \\
1964-86 \\
1965-86\end{array}$ \\
\hline $\begin{array}{l}\emptyset 733884 \emptyset \\
\varnothing 733896 \emptyset \\
673396 \varnothing \emptyset \\
\varnothing 733961 \varnothing \\
\varnothing 733916 \varnothing\end{array}$ & $\begin{array}{l}\text { Mountain Fk nr Smithville } \\
\text { Broken Bow Lake nr Broken Bow } \\
\text { Mountain Fk nr Eagletown } \\
\text { Mountain Fk blw Eagletown } \\
\text { Little R nr Cerrogordo }\end{array}$ & $\begin{array}{l}\text { QWMP } \\
\text { RESP } \\
\text { STRP } \\
\text { QWP } \\
\text { QWP }\end{array}$ & $\begin{array}{l}\text { CNP } \\
C P \\
C P\end{array}$ & $\begin{array}{l}\text { RES } \\
\text { DAR }\end{array}$ & $\begin{array}{l}c \\
c\end{array}$ & $\begin{array}{l}764 . \\
787 .\end{array}$ & $\begin{array}{l}1976-79 \\
1968- \\
1924-25,29- \\
1961-63 \\
1961-63\end{array}$ \\
\hline 67340600 & Little R $n r$ Horatio AR & QWMP & CNP & & & 2674 . & $1978-79$ \\
\hline
\end{tabular}

* Continuous streamflow records for this period.

** Some records in intervening years.

*** Some records may have been collected previously. 
Table 3.--Continuous and partial record ground-water level sites currently measured in Oklahoma.

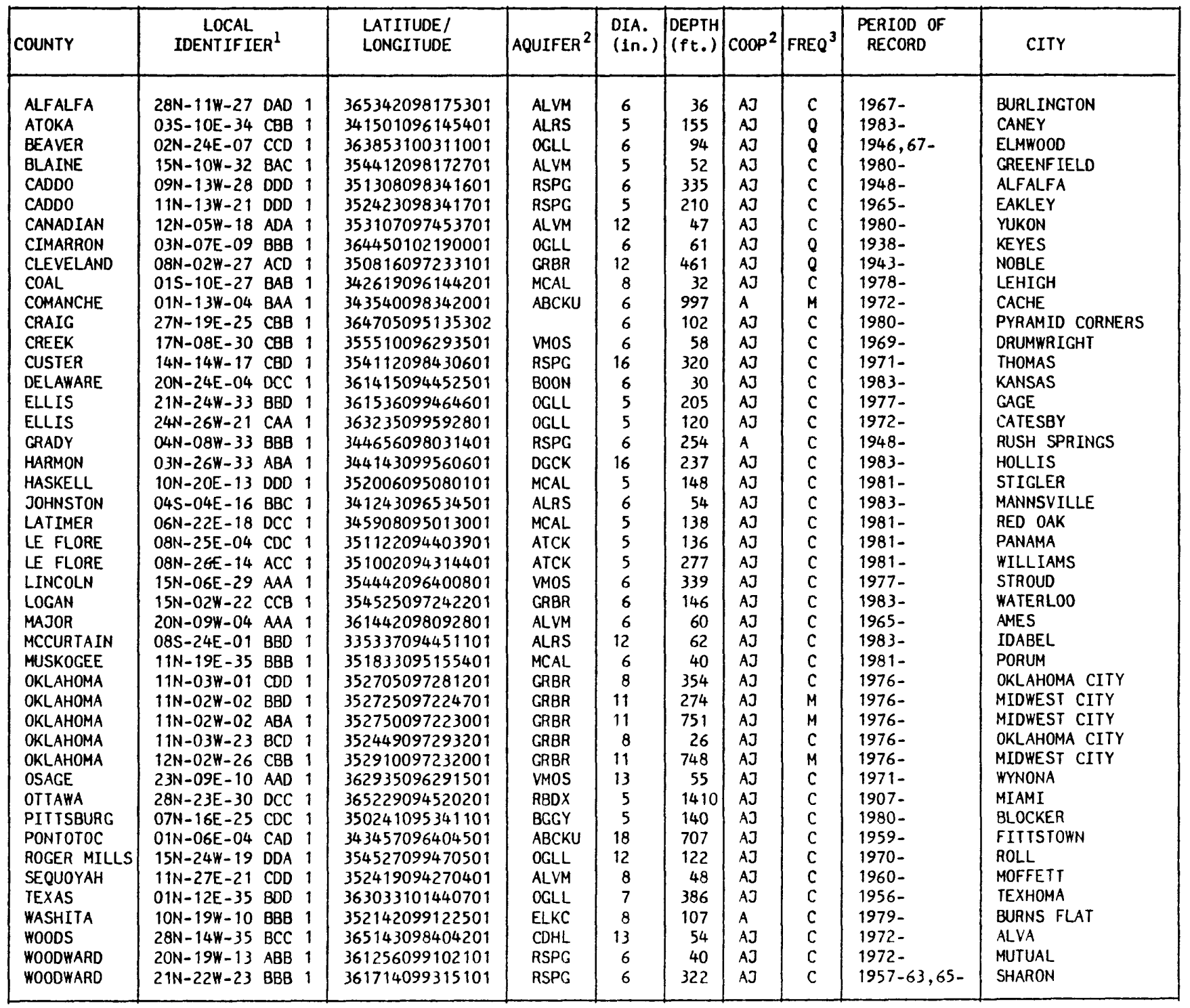

1--LOCAL IDENTIFIER gives township, range, section number, and quarter-quarter section. (see sketch)

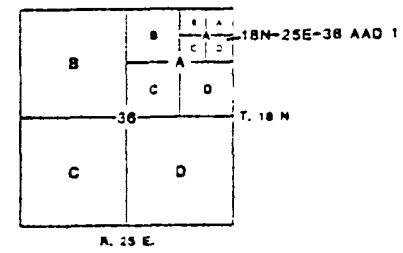

2--Codes for AQUIFER and COOP are given on last page of report.

${ }^{3}$--FREQ is frequency that water level is measured $(C)$-continuous, $(Q)$-quarterly, and $(M)$-monthly. 


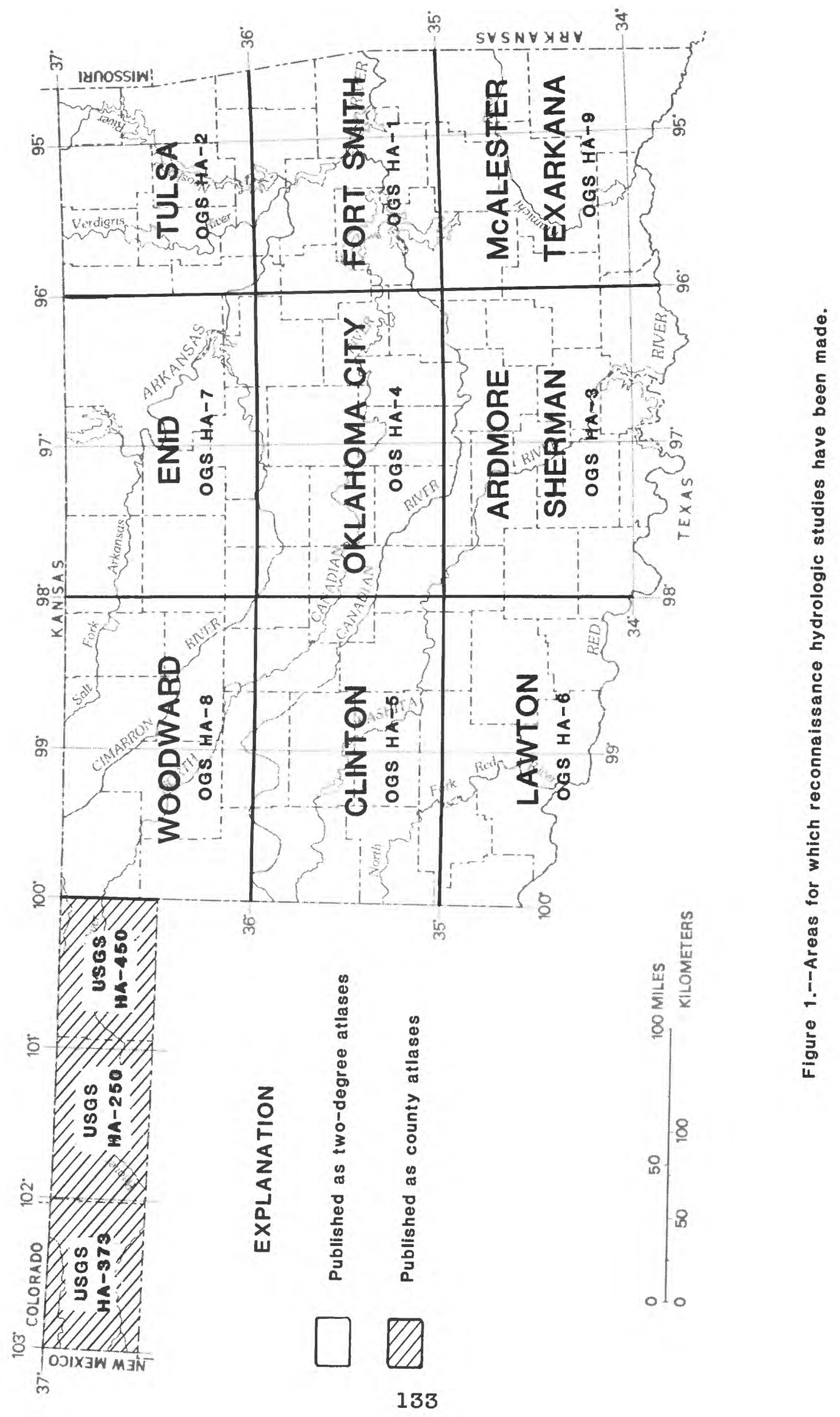




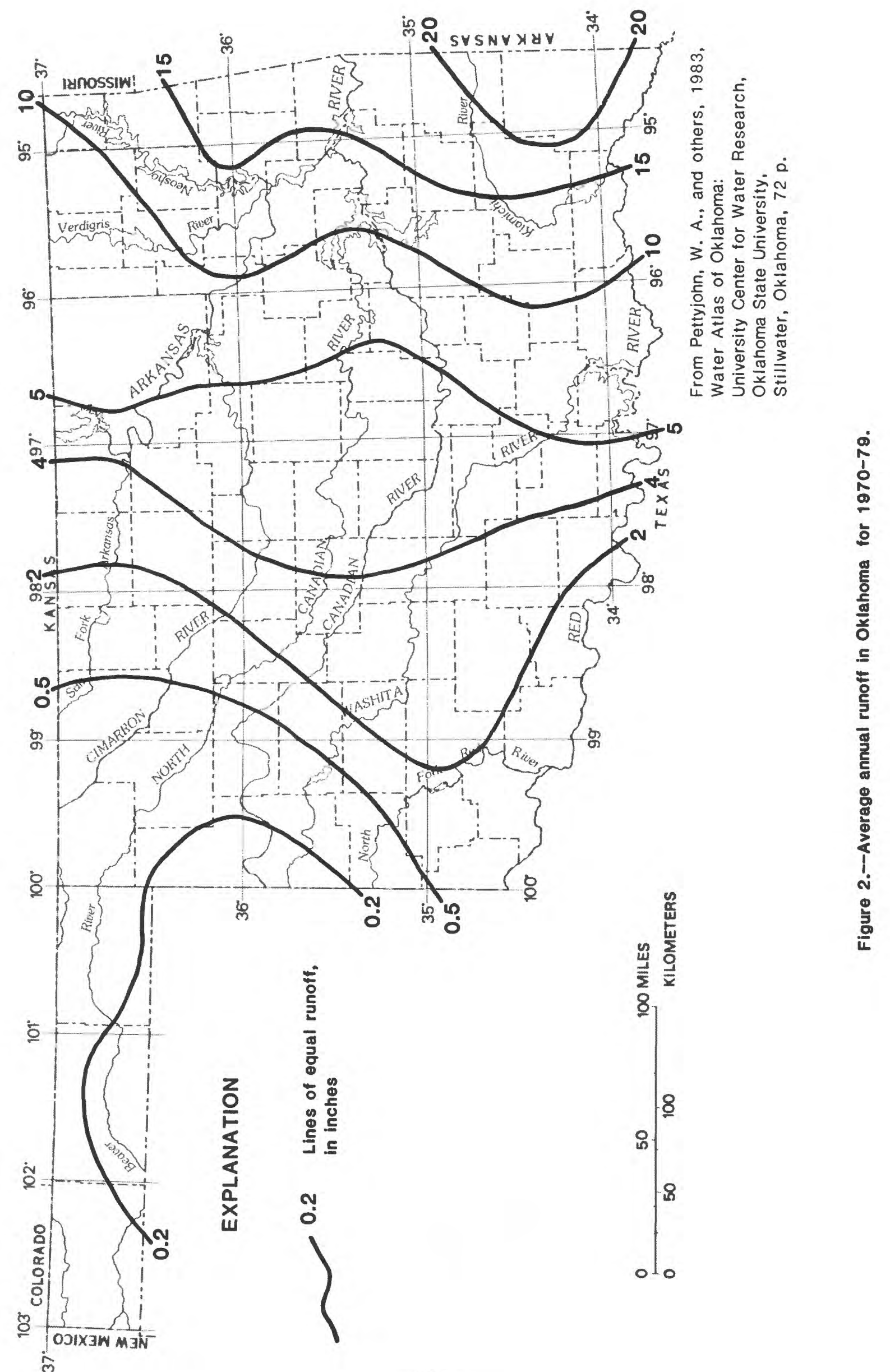




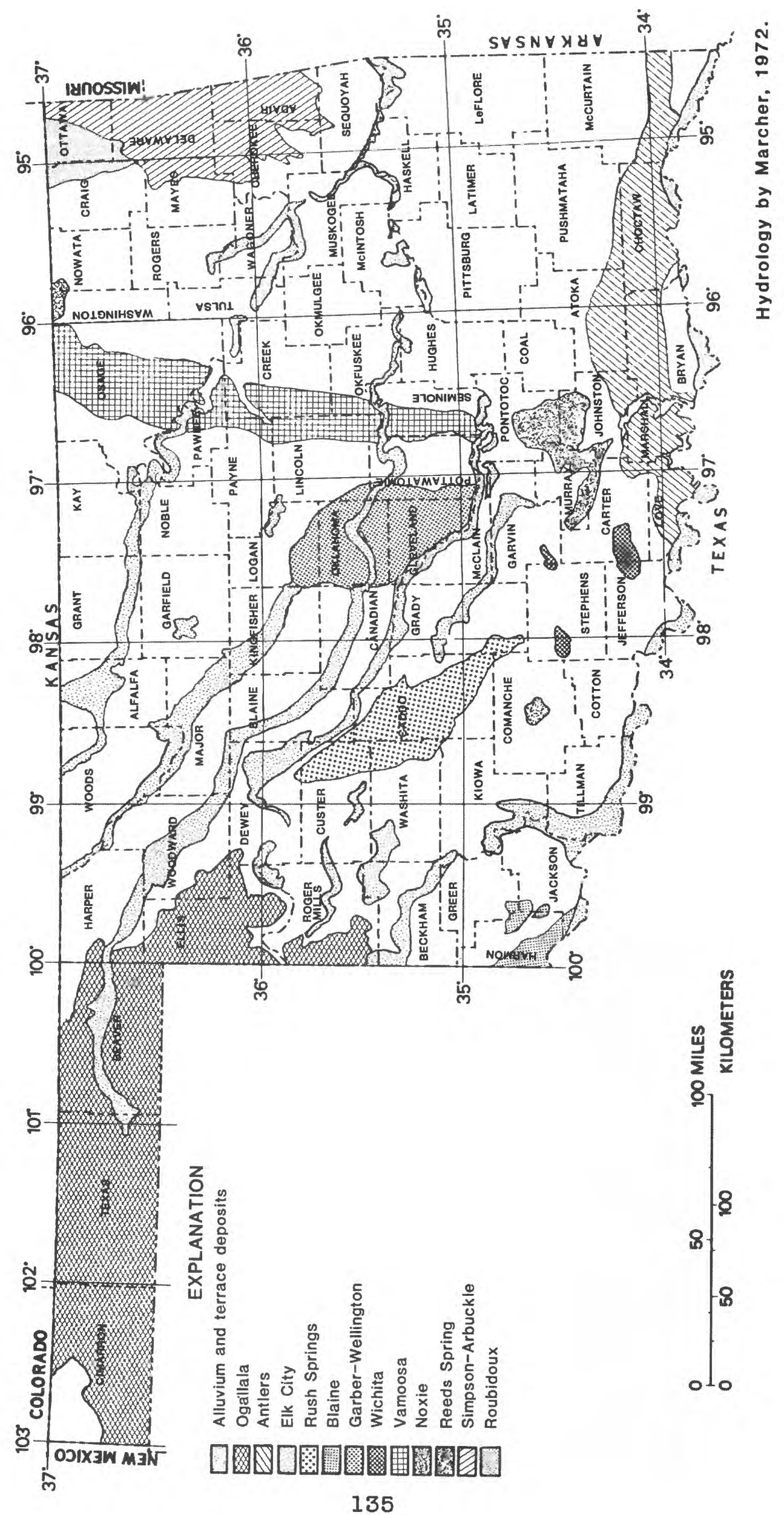

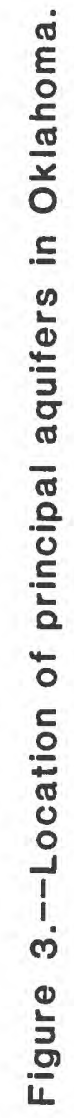




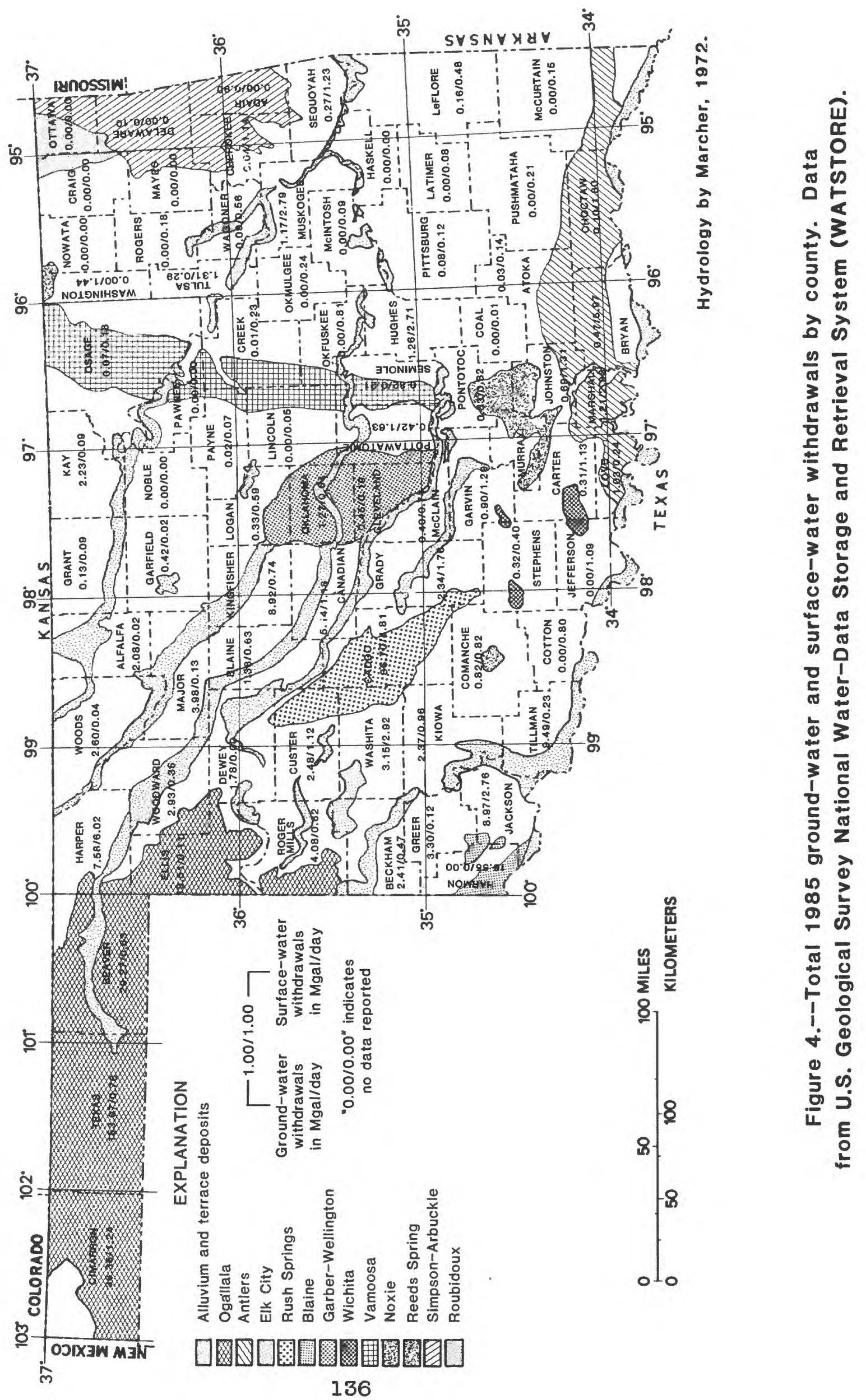




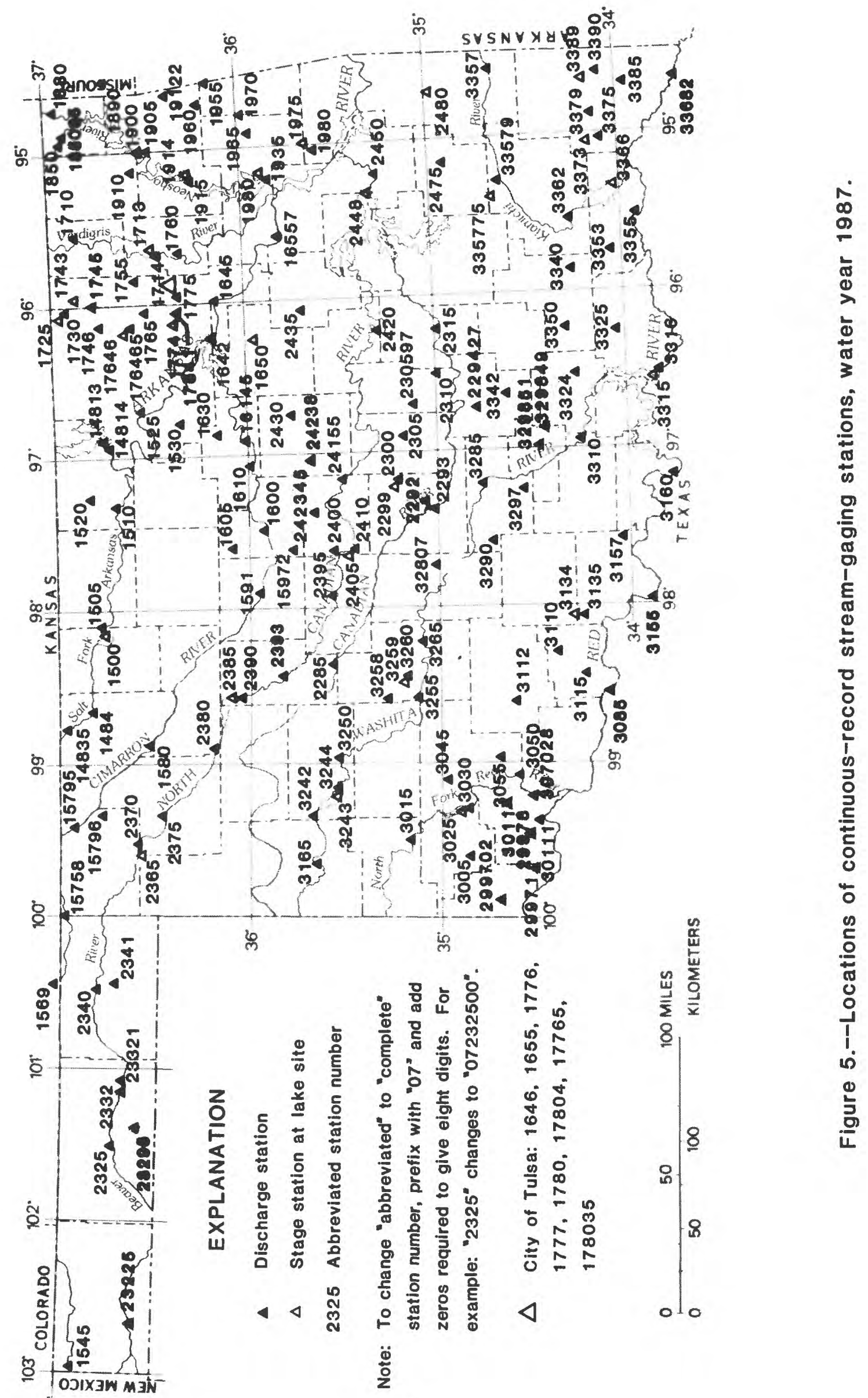

iे 


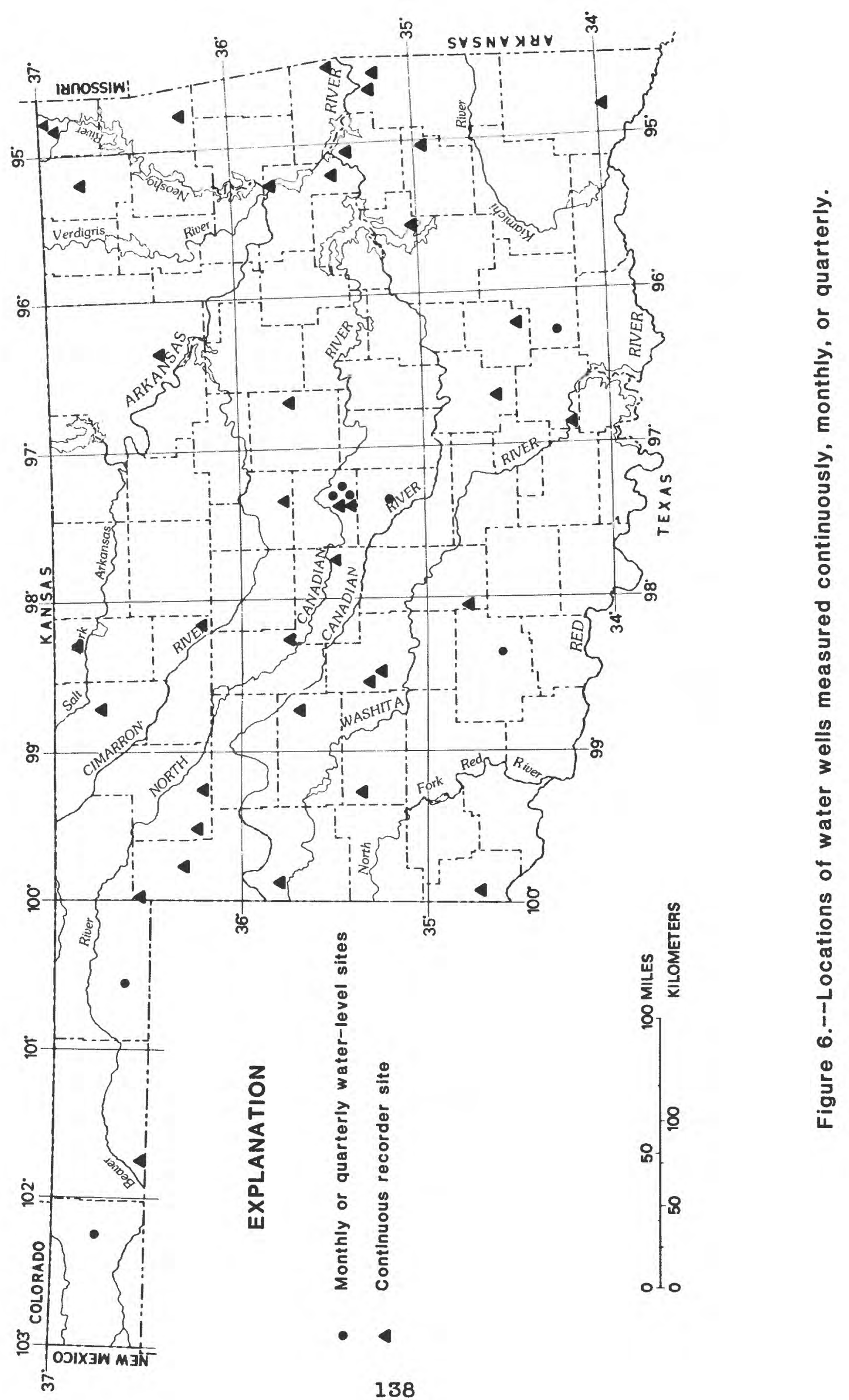




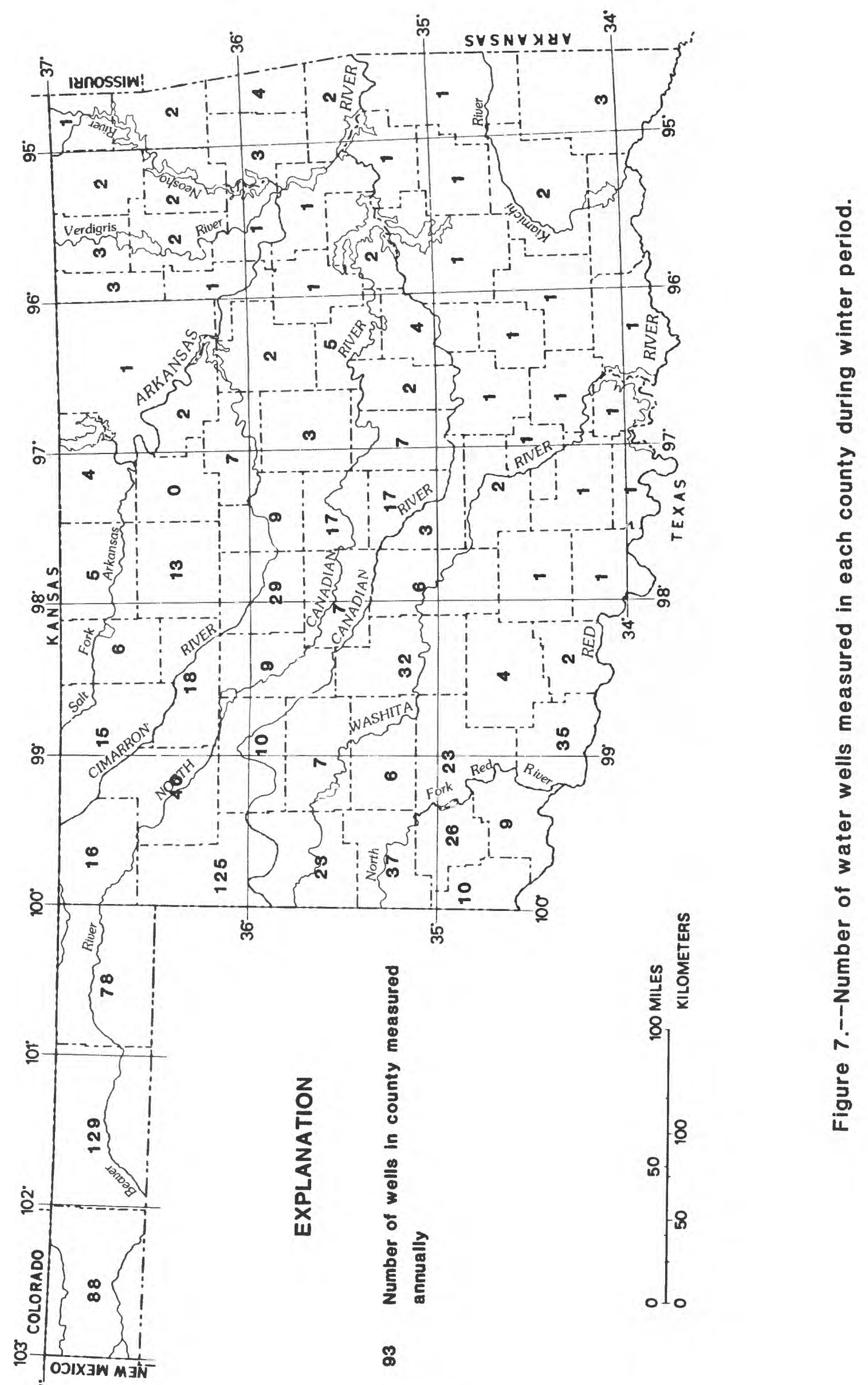




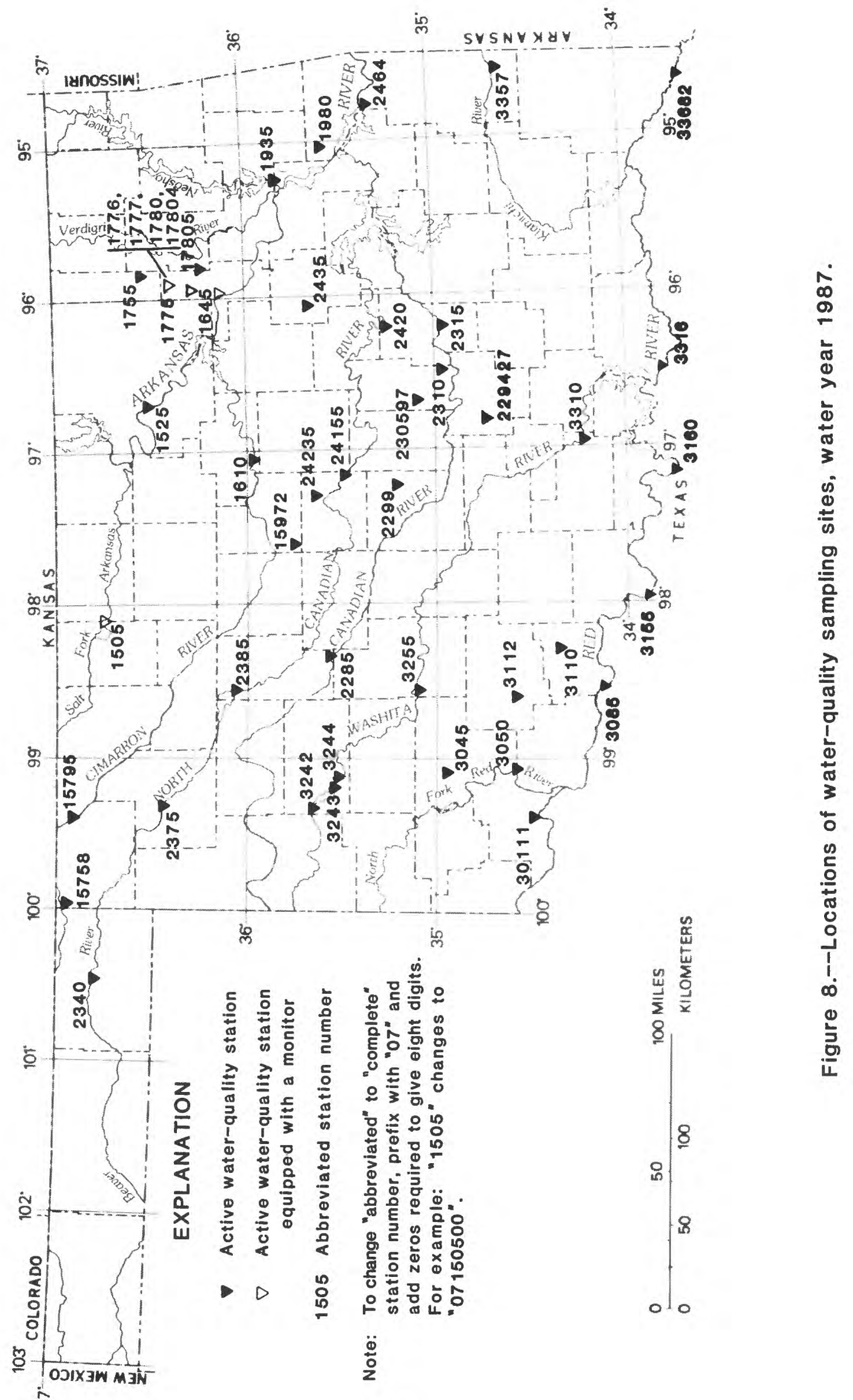




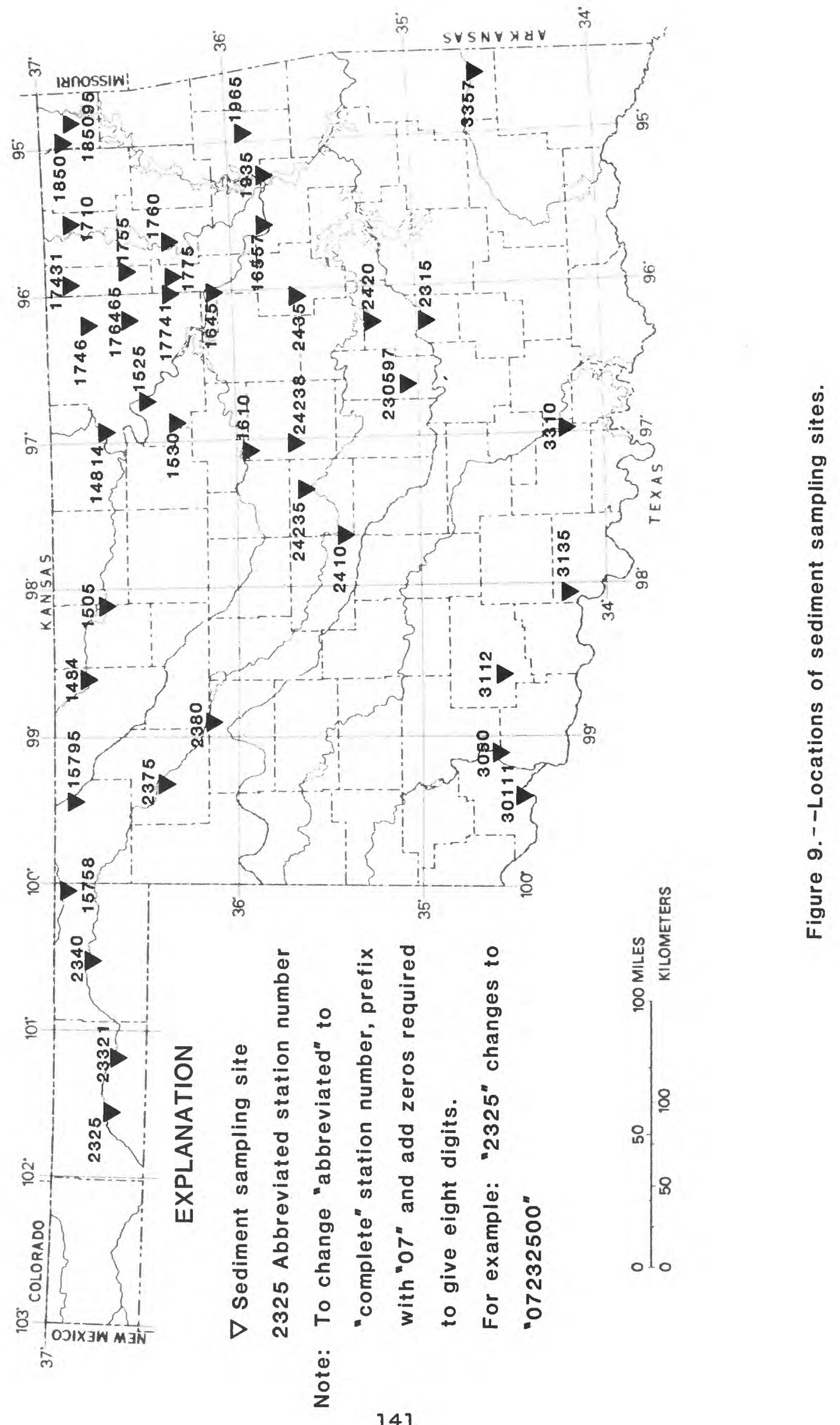

